\title{
Comprehensive Modernization of Firearm Discharge Residue Analysis; Advanced Analytical Techniques, Complexing Agents, and Tandem Mass Spectrometry
}

\author{
Brittany Louise Stevens
}

Follow this and additional works at: https://researchrepository.wvu.edu/etd

\section{Recommended Citation}

Stevens, Brittany Louise, "Comprehensive Modernization of Firearm Discharge Residue Analysis; Advanced Analytical Techniques, Complexing Agents, and Tandem Mass Spectrometry" (2017). Graduate Theses, Dissertations, and Problem Reports. 6726.

https://researchrepository.wvu.edu/etd/6726

This Dissertation is protected by copyright and/or related rights. It has been brought to you by the The Research Repository @ WVU with permission from the rights-holder(s). You are free to use this Dissertation in any way that is permitted by the copyright and related rights legislation that applies to your use. For other uses you must obtain permission from the rights-holder(s) directly, unless additional rights are indicated by a Creative Commons license in the record and/ or on the work itself. This Dissertation has been accepted for inclusion in WVU Graduate Theses, Dissertations, and Problem Reports collection by an authorized administrator of The Research Repository @ WVU.

For more information, please contact researchrepository@mail.wvu.edu. 
Comprehensive Modernization of Firearm Discharge Residue Analysis; Advanced Analytical Techniques, Complexing Agents, and Tandem Mass Spectrometry

\title{
Brittany Louise Stevens
}

\author{
Dissertation submitted to the \\ Eberly College of Arts and Sciences \\ at West Virginia University \\ in partial fulfillment of the requirements for the degree of \\ Doctor of Philosophy in Chemistry \\ Suzanne Bell, Ph.D., Chair \\ Harry Finklea, Ph.D. \\ Glen Jackson, Ph.D. \\ Keith Morris, Ph.D. \\ Stephen Valentine, Ph.D.

\section{Eugene Bennett Department of Chemistry}

\section{Morgantown, West Virginia \\ 2017}

Key Words: gunshot residue, firearm discharge residue, ion mobility spectrometry, thermal desorption, ESI, complexing, crown ether

Copyright 2017 Brittany Louise Stevens 


\section{Abstract \\ Comprehensive Modernization of Firearm Discharge Residue Analysis; Advanced Analytical Techniques, Complexing Agents, and Tandem Mass Spectrometry \\ Brittany Louise Stevens}

The use of firearm discharge residue (FDR) evidence has been on the decline as a result of instrumental and analytical limitations and the inability to evaluate and assign evidentiary value. To utilize FDR evidence to its fullest extent, detection methods exploiting modern advancements in instrumentation must be explored and developed. Research has been performed in an effort to modernize FDR analysis but to date nothing has been implemented or found widespread use in forensic laboratories. This research investigated three analytical techniques for the detection of FDR; (1) ion mobility spectrometry (IMS), (2) thermal desorption gas chromatography mass spectrometry (TD-GC/MS), and (3) electrospray ionization tandem mass spectrometry $\left(E S I-M S^{n}\right)$. An IMS method for organic gunshot residues was validated and then employed in a population study to determine shooter from non-shooters by analyzing samples taken from a subject's hands. Peaks corresponding to three organic gunshot residue (OGSR) compounds were detected in approximately $70 \%$ of shooter samples. Matrix issues associated with the swab material and the hands of subjects inherently complicated spectra. The results show a need of a pattern-based analysis rather than relying on peak identification for characterizing shooters vs. non-shooters hand swabs.

The next phase of this research was prompted by the need to develop confirmatory detection methods and reach lower limits of detection. A thermal separation probe was affixed to a GC/MS and allowed direct analysis of hand swabs without any prior sample preparation. A method was developed and authentic shooter swabs were analyzed. Although, three OGSR compounds were detected in $14-81 \%$ of authentic samples, additional work remains before the technique can begin to be implemented. Finally, experiments on detecting gunshot residue with ESI-MS ${ }^{\mathrm{n}}$ via complexing with a macrocyclic host were performed. The macrocyclic host, 15crown-5, was evaluated for complexation with known GSR metals. Foundational parameters were established and single and double ligand complexes were identified using isotopic ratios and fragment ions. Mass spectral intensities were used to determine the binding selectivities of the metals to the crown ether and in turn the preferential binding of the target metals. Additionally, preliminary molecular modeling provided insight into some experimental observations. Overall, three methods were evaluated in an effort to modernize the analysis of firearm discharge residues and in doing so increase the evidentiary value. IMS and thermal desorption GC/MS proved adequate as screening methods for OGSR and while additional work is required, ESI-MS ${ }^{\mathrm{n}}$ proved promising for detecting complexed GSR metals. The advantage of coupling ESI-MS ${ }^{\mathrm{n}}$ and complexation is that it allows for the dual detection of OGSR and GSR. While modernizing analysis is key to increasing the evidentiary value it is apparent that coupling the detection of OGSR and GSR is the future of FDR analysis. 


\section{Dedication}

Dedicated to my mom and husband, without you this would not have been possible.

Do not deny yourself accomplishment. 


\section{Acknowledgements}

Graduate school has been a trying and stressful journey. I would not have made it this far on my own and with that I would like to acknowledge the following groups and individuals:

\section{Family and Friends:}

I am very lucky to have such wonderful people supporting me and pushing me to achieve my dreams. My friends and family mean the world to me and I am ecstatic to be able to share this accomplishment with them.

Justin and Mom, you have been so great throughout this journey, providing support, encouraging words, and hugs when I needed them most. Justin, thank you for being there with me through the ups and downs and taking care of everything when I needed to checkout and focus on school. You and Bub have kept me sane and focused from day one. Mom, I channel your drive to succeed and refusal to accept failure often when I am struggling. I can't thank you enough for pushing me to accomplish this dream even when I lost sight.

Liz Dearth, you are such a wonderful person and friend. God blessed me by putting you in my life at exactly the right moment. You have always provided constant encouragement and your daily notes helped to brighten up the dreary days. You made sure I remembered to laugh often and enjoy life.

The past and current members of the Bell Research Group at West Virginia University, thank you for your help, advice, and, most importantly, your friendship and support. I hope the impact I made on your lives in as great as the one you made on mine. Corey Nida, Lee Greenawald, and Holy McCall, you were my go-to people when I needed reminded of why I was pursuing this degree. Thank you for checking in on me periodically. Your journeys and successes provided me constant inspiration and encouragement. Stephen Raso, Kristin Kelly, and Bill Feeney, you've made life in lab way more enjoyable over the past few years. I would also like to acknowledge and thank the undergraduates who helped me over the past 5 years. James Stewart, Ryan Dross, Katelyn Bustin, Lauren Seitzinger, Kirsten Adams, and Sydney Brooks, your assistance and hard work is so greatly appreciated.

\section{Faculty:}

Becky Secrist, you are an amazing individual and have impacted so many graduate student's lives. The department is very lucky to have you. Thank you for everything you have helped me with over the years. I am going to miss your smiling face and cheerful hellos.

Last, but certainly not least, I would like to thank every one of my committee members, Dr. Suzanne Bell, Dr. Harry Finklea, Dr. Glen Jackson, Dr. Stephen Valentine, and Dr. Keith Morris for reviewing my dissertation and serving on my graduate committee. Dr. Bell, thank you for pushing and pushing me up and over the mountain of graduate school. 


\section{Table of Contents}

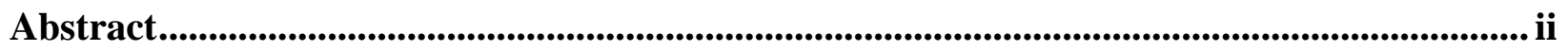

Acknowledgements ......................................................................................................................... iv

List of Figures...................................................................................................................................... viii

List of Tables ............................................................................................................................... $\mathrm{x}$

List of Abbreviations, Symbols, and Nomenclature .................................................................. xi

Chapter 1: Introduction ....................................................................................................................................... 1

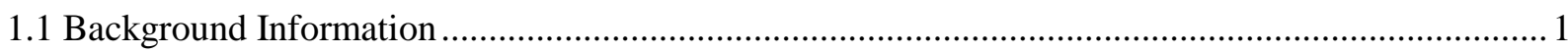

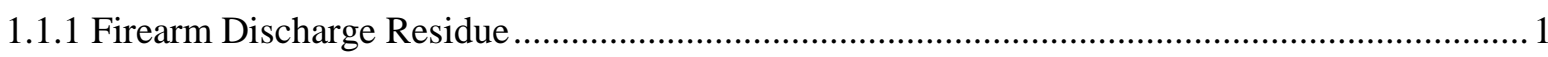

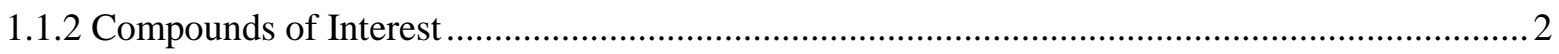

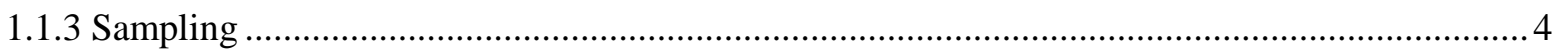

1.1.4 Current Forensic Analytical Methodology .......................................................................

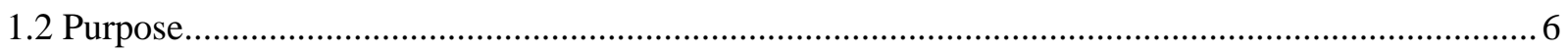

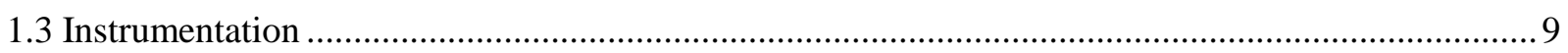

1.3.1 Ion Mobility Spectrometry (IMS) …………………................................................

1.3.2 Gas Chromatography - Mass Spectrometry (GC/MS) …………………………………...... 11

1.3.3 Electrospray Ionization Tandem Mass Spectrometry (ESI-MS/MS)........................................ 13

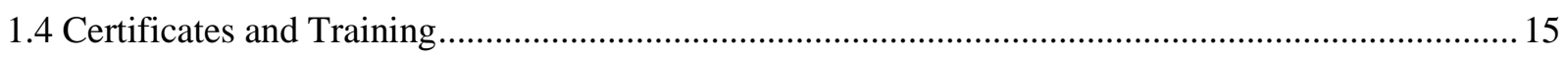

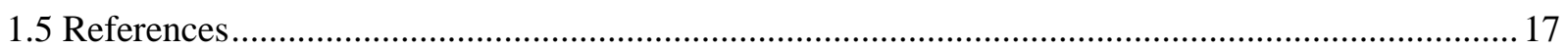

Chapter 2: Evaluation and validation of Ion Mobility Spectrometry for Presumptive Testing Targeting the Organic Constituents of Firearms Discharge Residue ${ }^{1}$....................... 19

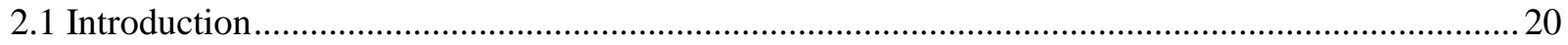

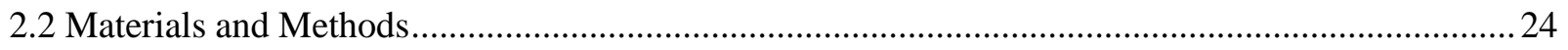

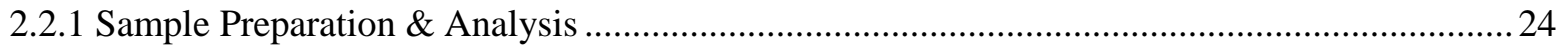

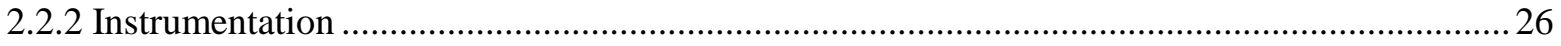

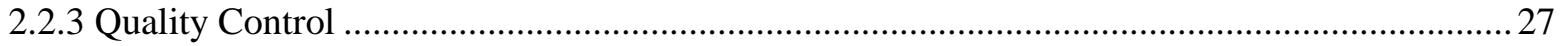

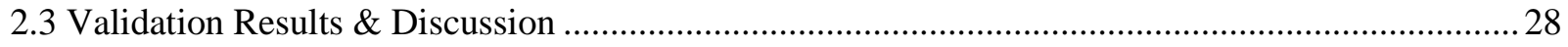

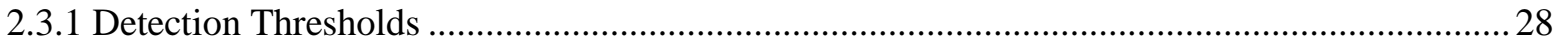

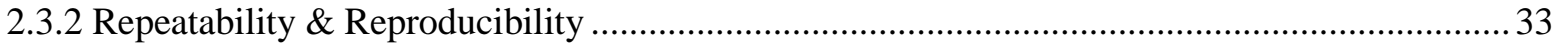

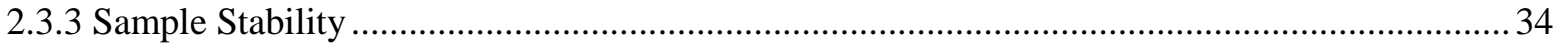

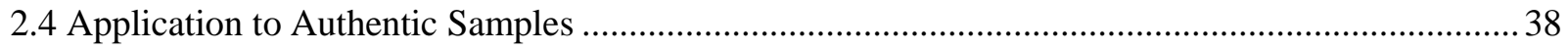

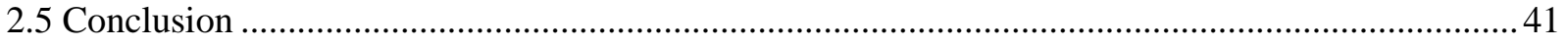




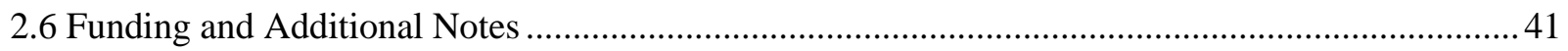

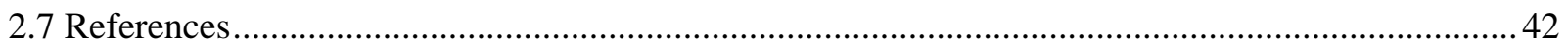

Chapter 3: Initial evaluation of inlet thermal desorption GC-MS analysis for organic

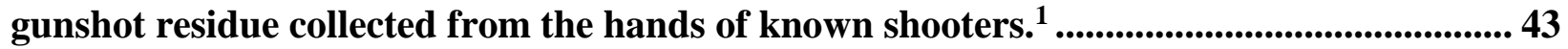

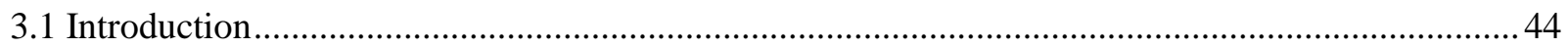

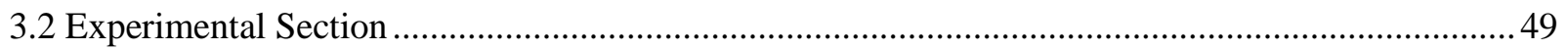

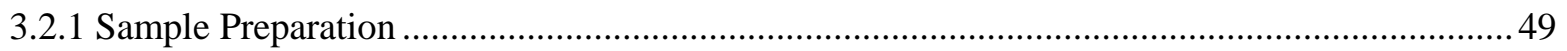

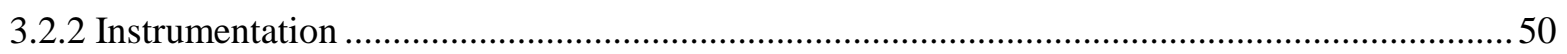

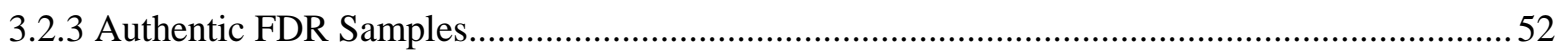

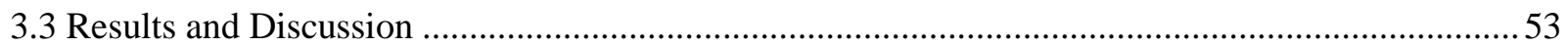

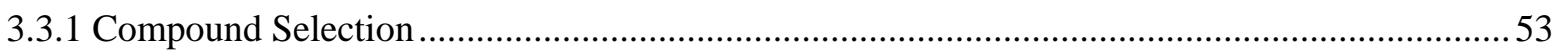

3.3.2 Instrumentation and Procedure Optimization ...................................................................... 54

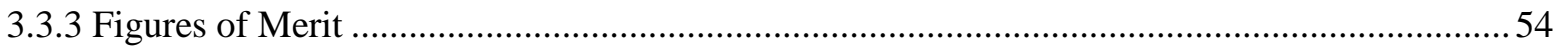

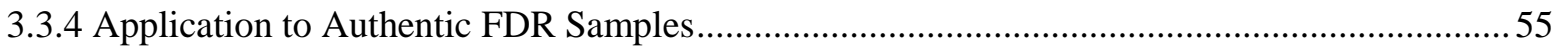

3.3.5 Use of Surrogates and Statistical Interpretation of Results..................................................59

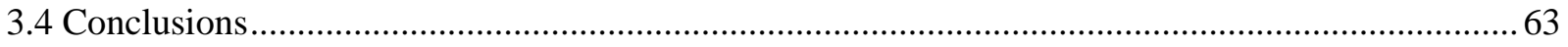

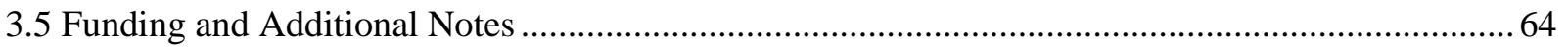

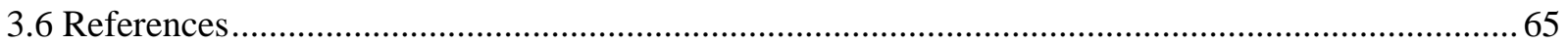

Chapter 4: Evaluation of host-guest complexation for the analysis of elemental firearm discharge residue with electrospray tandem mass spectrometry (QqQ)........................... 67

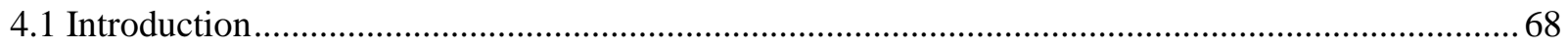

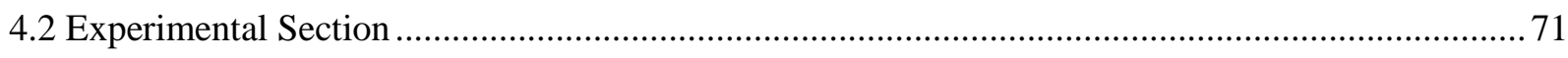

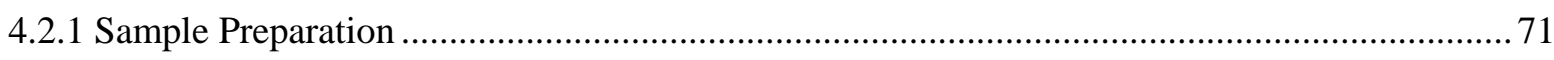

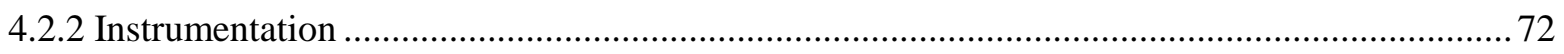

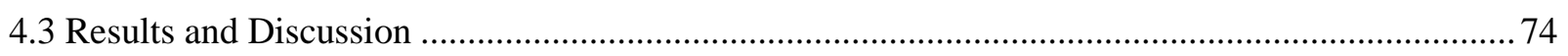

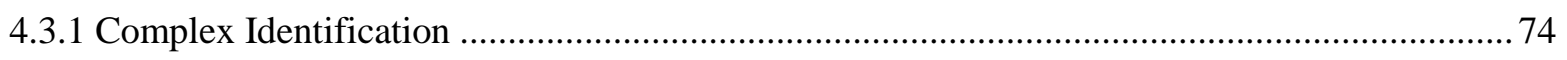

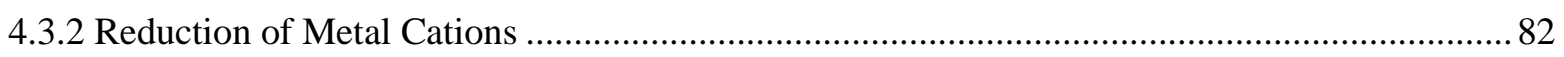

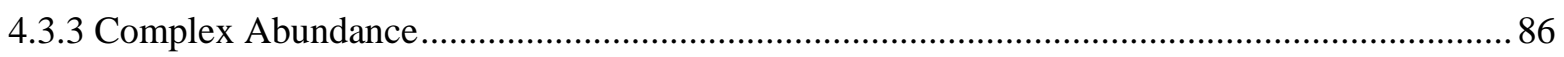

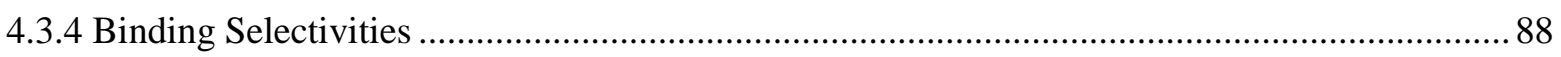

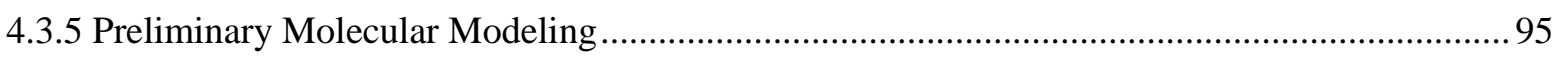

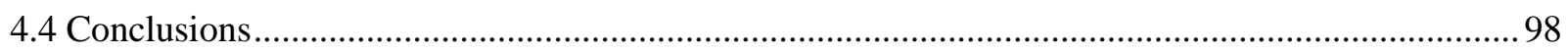

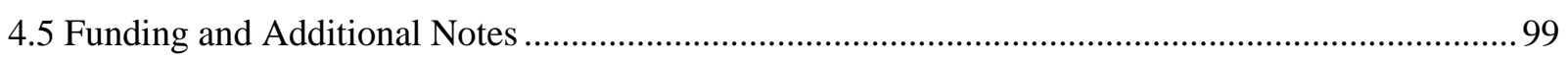

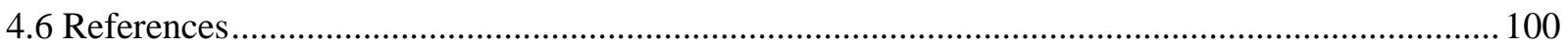

Chapter 5: Conclusions and Future Directions......................................................... 103

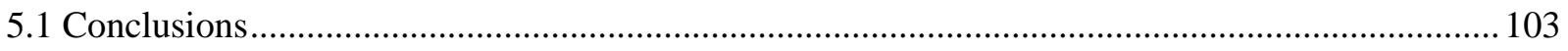




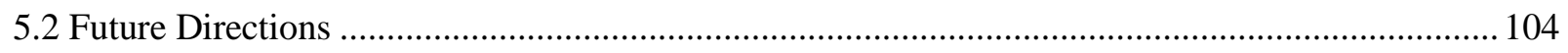

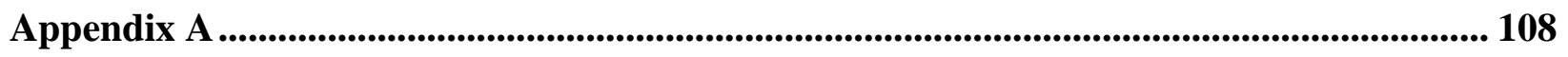

Appendix B ..................................................................................................................................... 113

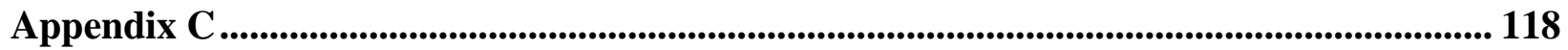




\section{List of Figures}

Figure 1.1: Timeline of OGSR research performed in our laboratory $\ldots \ldots \ldots \ldots \ldots \ldots \ldots \ldots . . \ldots$

Figure 1.2 Schematic of an ion mobility spectrometer............................... 10

Figure 1.3 Schematic of a GC/MS system. ........................................ 12

Figure 1.4 Block diagram of a triple quadrupole mass spectrometer. .................... 14

Figure 2.1 Physical and chemical evidence produced by a firearm discharge.......... 20

Figure 2.2 Control charts for the Sabre ${ }^{\circledR}$ handheld the horizontal lines indicate the calculated warning and control limits (AMP - amplitude, LWL - lower warning limit, LCL, lower control limit, UWL - upper warning limit, UCL - upper control limit)................ Figure 2.3 Full range mobility spectra $(1-20 \mathrm{~ms})$ obtained with the Ionscan ${ }^{\circ}$. The $\mathrm{x}$-axis is drift time in milliseconds $(\mathrm{ms})$ and the y-axis is the peak intensity in millivolts $(\mathrm{mV}) \ldots \ldots \ldots$ Figure 2.4 Zoomed to MC mobility peak in which the detection threshold is indicated......... Figure 2.5 Average mobility spectra obtained with the Ionscan ${ }^{\circledR}$ in which stability is a function of storage conditions. Solid lines indicate samples stored in the freezer (FR) at $26^{\circ} \mathrm{C}$ whereas dashed lines are samples stored at room temperature........................ Figure 2.6 Average maximum peak intensity $(n=3)$ for DMT and DPA; samples stored in freezer. The $\mathrm{x}$-axis is the number of days elapsed since preparation and the $\mathrm{y}$-axis is the intensity. The error bars correspond to the $95 \%$ confidence interval for $n=3 \ldots \ldots \ldots \ldots \ldots$. Figure 2.7 Average maximum peak intensity $(n=3)$ for MC and EC; samples stored in freezer. Axes and error bars are as stated in Figure 2.6....

Figure 2.8 Average maximum peak intensity $(n=3)$ for all four target compounds; samples stored at room temperature. Axes and error bars are as stated in Figure 2.6.................. Figure 2.9 Summarized results for authentic samples analyzed with the Sabre® (handheld/portable) instrument.

Figure 2.10 Summarized results for authentic samples analyzed with the Ionscan ${ }^{\circledR}$

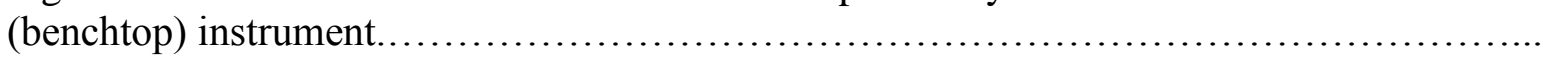

Figure 2.11 Spectra indicating the differences between hands of shooters and other collected

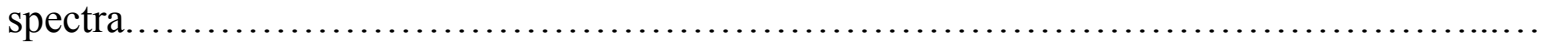

Figure 3.1 Deposition of firearm discharge residue including GSR and OGSR on the surface

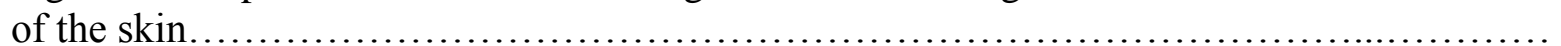

Figure 3.2 Cut CapSure ${ }^{\circledR}$ VP swab measuring approx. 3 x $1.5 \mathrm{~cm}$ used as sampling media... Figure 3.3A Thermal separation probe and sample vial situated in the sample loading apparatus. B. Thermal desorption unit affixed to the inlet of the GC system. Both are commercially available through Agilent Technologies Inc., Santa Clara, CA, USA............ Figure 3.4 In-house fabricated swab holder used for authentic sample collection................ Figure 3.5A Surrogate compound 1, bivariate plot with histograms $(n=70) \ldots \ldots \ldots \ldots \ldots . . . .$. Figure 3.5B Surrogate compound 2, bivariate plot with histograms $(\mathrm{n}=70) \ldots \ldots \ldots \ldots \ldots .$. Figure 4.1A-F Peaks identified as having the correct isotopic signature for the metal complexes of interest. Note - Spectra scan range is from 50-1,000 Da the figures are zoomed in to the region of interest. Additionally, the $y$-axis (intensity) varies between

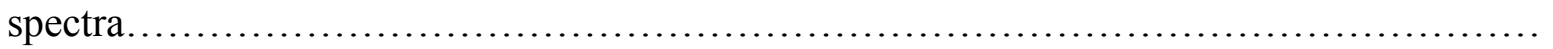

Figure 4.2 A \& B Diluted 2:1:1 potassium and lead solution used for potassium complex isotopic signature confirmation. Note - Spectrum range is from $50-1000 \mathrm{Da}$ and the

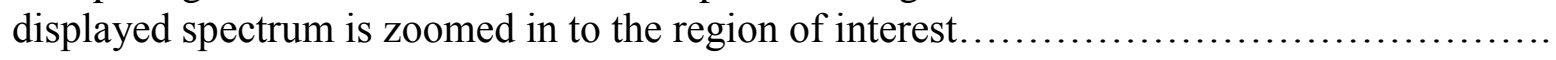

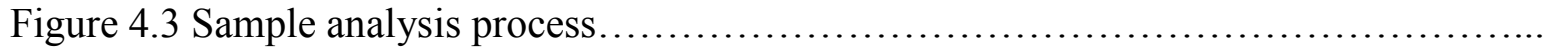


Figure 4.4 MS/MS spectrum of precursor ion $m / z, 200\left(\mathrm{BaNO}_{3}\right)$ in which the ${ }^{138} \mathrm{Ba}$ isotope is observed.

Figure 4.5 A. Q1(MS) spectrum of the $\left[15-5+\mathrm{Pb}_{-} \mathrm{NO}_{3}\right]^{+}$complex in which the oxidation state of $\mathrm{Pb}$ is $\mathrm{a}+2$. B. Q3(MS/MS) spectrum of the parent ion $m / z 490\left(\left[15-5+\mathrm{Pb}-\mathrm{NO}_{3}\right]^{+}\right)$ complex in which the oxidation state of $\mathrm{Pb}$ is a $+1 \ldots$

Figure 4.6 Calculated experimental \% $\mathrm{T}[\mathrm{M}]$ of the 2:1:1 and 3:1:1:1 mixtures which were directly translated into binding selectivities of the target metals.

Figure 4.7 Minimum energy structures of 15-crown-5 and the metal ions of interest; the yellow sphere and dark grey spheres represent the $\mathrm{M}^{+\mathrm{n}}$ ions. The red (oxygen), white (hydrogen), and light grey (carbon) spheres comprise the crown ether complex. Note that the depicted metal ions are not to scale with their ionic radii.

Figure 5.1Depiction of the next phase of FDR research in our laboratory 


\section{List of Tables}

Table 1.1 Target compounds and elements and their sources along with their location within this text.......................................................................

Table 2.1 Target compounds and associated material. $\mathrm{Vp}$ is vapor pressure in $\mathrm{mm} \mathrm{Hg}$ at $25^{\circ} \mathrm{C}$ with sources noted below.

Table 2.2 Operating parameters and conditions of the ion mobility spectrometers used for this study.

Table 2.3 Established IMS instrument critical thresholds a. is the Sabre's ${ }^{\circledR}($ handheld) \& b. is the Ionscan's ${ }^{\circledR}$ (benchtop) thresholds

Table 2.4 Amount deposited compared to estimated detection thresholds for the compounds of interest based on the established critical thresholds..................................... Table 2.5 Repeatability (intra-) and reproducibility (inter-) of peak intensities (mv, \%RSD).. Table 3.1 Final compound target list with corresponding abbreviations and ions used in the developed SIM method where the quantifier ion is the most intense ion..................... Table 3.2 Instrument and method LOD values for target compounds.

Table 3.3 Example authentic shooting sample data for DPA including the 3 of the 4 criteria applied to the peaks of interest; ion presence, ion ratio, and $\mathrm{S} / \mathrm{N}$. All retention times for both

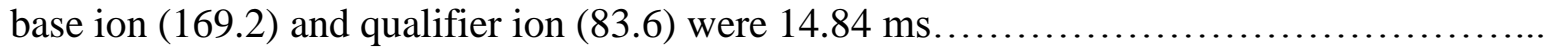
Table 3.4 Overall authentic shooting sample results. Values are by overall swab not by piece.

Table 3.5 Results for all pieces of shooting swabs when analyzed via the contour plots established from criteria data for each compound compared to flowchart results. $(n=59) \ldots \ldots$ Table 4.1 Electrospray ion source conditions for all mass spectrometry experiments.......... Table 4.2 The atomic masses and natural abundances of each isotope of the metals of interest used to calculate $\mathrm{m} / \mathrm{z}$ values and determine isotopic patterns of the crown ether metal complexes.

Table 4.3 Ions observed for the 1:1 crown ether metal mixtures and the fragment ion used to confirm the identification of the complex in which " $\mathrm{L}$ " represents 15-crown-5.

Table 4.4 Observed complexes for each target metal in Q1 and Q3 spectra. The m/z values listed are those associated with the most abundant isotope.

Table 4.5 Calculated relative percent abundance for each metal complex. Values were calculated using average intensity $(n=3)$ of the most abundant isotope with the exception of potassium (second most abundant isotope).

Table 4.6 The peaks used to calculate the \% $[\mathrm{M}]$ for each method utilizing the $2: 1: 1$ mixture of 15-crown-5, copper and barium as an example. The peaks used for the other mixtures are located in Appendix C.

Table 4.7 Calculated experimental and theoretical percent total metal complex (\% $[\mathrm{M}])$ for the metals in the $2: 1: 1$ mixtures of 15 -crown-5. 


\section{List of Abbreviations, Symbols, and Nomenclature}

\%RSD
$\% \mathrm{~T}[\mathrm{M}]$
2,4-DNT
2-NDPA
4-NDPA
${ }^{63} \mathrm{Ni}$
A
AC
ACS
APCI
Ba
CAD
Carb
CE
CEP
CID
CITI
cm
CMV
CRM
Cu
CUR
CXP
d
Da
DBP
DC
DMT/DMP
DP
DPA
DtBP
E
EC
EDS
EP
EPA
ESI
eV
FDR
GC
GS1
GS2
GSR
H

Percent Relative Standard Deviation

Percent Total Metal Complex

2,4-dinitrotoluene

2-nitrodiphenylamine

4-nitrodiphenylamine

Nickel Isotope 63

Angstrom

Alternating Current

American Chemical Society

Atmospheric Pressure Chemical Ionization

Barium

Collision Activated Dissociation

Carbazole

Collision Energy

Collision Cell Entrance Potential

Collision Induced Dissociation

Collaborative Institutional Training Initiative

Centimeter

Capillary Microextraction of Volatiles

Charged-residue model

Copper

Curtain Gas

Collision Cell Exit Potential

Distance

Dalton

Dibutyl Phthalate

Direct Current

Dimethyl Phthalate

Declustering Potential

Diphenylamine

2,6-di-tert-butylpyridine

Electric Field

Ethyl Centralite

Energy Dispersive X-ray Spectrometry

Entrance Potential

Environmental Protection Agency

Electrospray Ionization

Electron Volt

Firearm Discharge Residue

Gas Chromatography

Gas 1

Gas 2

Gunshot Residue

Enthalpy 


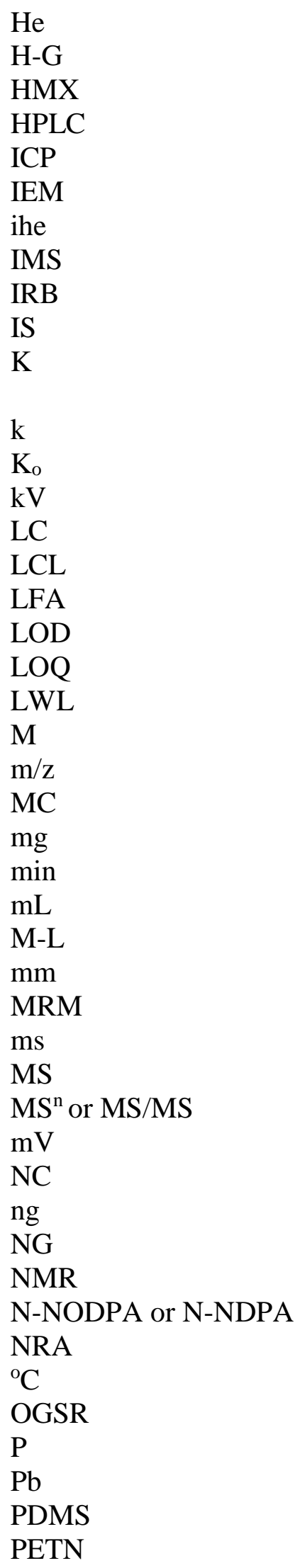

Helium

Host Guest

1,3,5,7-Tetranitro-1,3,5,7-tetrazocane

High Performance Liquid Chromatography

Inductively Couple Plasma

Ion Evaporation Model

Interface Heater

Ion Mobility Spectrometry

Institutional Review Board

Ionspray Voltage

Potassium, Ion Mobility, Kelvin, or

Equilibrium Constant

Rate Constant

Reduced Mobility Coefficient

Kilovolt

Liquid Chromatography

Lower Control Limit

Lead Free Ammunition

Limit of Detection

Limit of Quantitation

Lower Warning Limit

Molarity

Mass to Charge Ratio

Methyl Centralite

Milligram

Minute

Milliliter

Metal Ligand

Millimeter

Multiple Reaction Monitoring

Millisecond

Mass Spectrometry

Tandem Mass Spectrometry

Millivolt

Nitrocellulose

Nanograms

Nitroglycerine

Nuclear Magnetic Resonance

$\mathrm{N}$-nitrosodiphenylamine

National Rifle Association

Degrees Celsius

Organic Gunshot Residue

Pressure

Lead

Polydimethylsiloxane

Pentaerythritol Tetranitrate 


\begin{tabular}{|c|c|}
\hline Ppg & polypropylene glycol \\
\hline ppm & Parts Per Million \\
\hline Q & Quadrupole 1 or 3 \\
\hline$q$ & Quadrupole 2 \\
\hline QA & Quality Assurance \\
\hline QC & Quality Control \\
\hline QqQ & Triple Quadrupole \\
\hline QToF & Quadrupole Time of Flight \\
\hline RDX & 1,3,5-Trinitroperhydro-1,3,5-triazine \\
\hline RF & Radio Frequency \\
\hline RMS & Root Mean Square \\
\hline Rt & Retention Time \\
\hline $\mathrm{s}$ & Second \\
\hline $\mathrm{S}$ or $\mathrm{s}$ & Standard Deviation \\
\hline $\mathrm{S} / \mathrm{N}$ & Signal to Noise Ratio \\
\hline $\mathrm{Sb}$ & Antimony \\
\hline SEM & Scanning Electron Microscopy \\
\hline SIM & Select Ion Monitoring \\
\hline SPME & Solid Phase Microextraction \\
\hline $\mathrm{T}$ & Temperature \\
\hline $\mathrm{t}_{1 / 2}$ & Half life \\
\hline TD & Thermal Desorption \\
\hline$t_{D}$ & Drift Time \\
\hline TEM & Temperature \\
\hline TNB & 1,3,5-Trinitrobenzene \\
\hline TNT & Trinitrotoluene \\
\hline ToF & Time of Flight \\
\hline TSP & Thermal Separation Probe \\
\hline UCL & Upper Control Limit \\
\hline UWL & Upper Warning Limit \\
\hline V & Volt \\
\hline $\mathrm{V}_{\mathrm{d}}$ & Drift Velocity \\
\hline VOC & Volatile Organic Compounds \\
\hline $\mathrm{Vp}$ & Vapor Pressure \\
\hline$X$ & Mean, Average \\
\hline$\mu \mathrm{ECD}$ & Micro-Electron Capture Detection \\
\hline$\mu g$ & Microgram \\
\hline$\mu \mathrm{L}$ & Microliter \\
\hline$\mu \mathrm{m}$ & Micrometer \\
\hline
\end{tabular}




\section{Chapter 1: Introduction}

\subsection{Background Information}

\subsubsection{Firearm Discharge Residue}

A cartridge used in small arms ammunition is comprised of four main components: a case, a projectile or bullet, primer, and propellant (powder). When a firearm is discharged the firing pin strikes the cartridge's shock sensitive primer activating it and progressively igniting the propellant within. The increased pressure from the gaseous products of burning propellant causes the expulsion of the bullet and with that a plume of a complex heterogeneous mixture of vapors and particles known as firearm discharge residue (FDR). The FDR mixture is comprised of inorganic particulates (GSR), and organic condensates and particles of unburnt and partially burnt propellant (OGSR collectively).

Inorganic particulates (GSR) are condensation products that are typically smooth and roughly spherical particulates containing metal oxides or sulfides. They are formed during the flash heating and condensation of compounds contained within the primer. Primer GSR particles range in size from $\sim 0.5$ to 5.0 microns and should not be confused with particles of un-burnt or partially burnt propellant (OGSR particulates) which will vary in size and shape depending on the original size and shape of the manufactured grains. Because OGSR particulates are not generated via condensation, they lack the distinctive spherical morphology found in GSR particulates and thus can be differentiated. In addition to GSR primer particles, elemental particles originating from bullet materials may be present. ${ }^{1}$

Deposition of both GSR and OGSR occurs on proximate surfaces surrounding the firearm discharge event. These surfaces include the hands, chest, shoulders, and face of the individual discharging the weapon with many variables dictating the amount of residue deposited; 
ammunition and weapon used, and environmental conditions during the firing event are key factors. In a recent study, the amount of OGSR deposited was estimated to be in the range of 90178 ng. $^{2}$ As for inorganic particulates, Shaffer and Yi recovered on average 389 particles (126 particles being classified as GSR particulates) with tape lifting and 60 with swabbing (3 particles classified as GSR particulates). ${ }^{3}$

Persistence of the deposited residues has proven to be an impediment to FDR collection, analysis, and interpretation. Persistence of particulate GSR evidence on the hands of shooters is limited to approximately 4 hours. ${ }^{4-6}$ In addition to the limited persistence, particulate GSR is readily lost via secondary transfer, minor physical activity, and/or hand washing. Secondary transfer can occur can through activities such as simple handshake making interpretation of the evidence more difficult. Organic gunshot residues (OGSR) have been found to be less prone to secondary transfer than the particulate evidence. This is believed to be due to the comprising analytes lipophilic nature facilitating adhesion to the skin. ${ }^{7}$ Persistence studies of common OGSR compounds on the hands of shooters have been previously studied in detail. ${ }^{7,8}$ It was found that these organic analytes, if not lost due to evaporation, permeate into the top layer of the skin known as the stratum corneum ${ }^{2,9}$ with the evaporation and absorption rates being compound dependent.

\subsubsection{Compounds of Interest}

Propellant and primer composition varies greatly between manufacturers and firearm caliber, resulting in an extensive list of potential target compounds both inorganic and organic. Recent review articles by Dalby ${ }^{10}$ and Taudte ${ }^{11}$ contain lists of a combined 68 different compounds that may contribute to organic gunshot residue. These organic compounds are energetics and additives that are commonly used in the manufacturing process of propellant 
powders and primer mixes. Energetic compounds will be present in the primer mix, as an initiating explosive, and the propellant. The most common propellants, smokeless double-base, contain two energetic compounds - nitrocellulose and nitroglycerin. Other energetics such as trinitrobenzene (TNB), TNT, PETN, HMX and RDX are also observed. Additives include stabilizers such as diphenylamine and its nitrated derivatives, flash suppressors such as nitrotoluene, stabilizers (ethyl- and methyl centralite) and plasticizers (methyl-, ethyl- and dibutyl phthalate). Furthermore, these additives may serve as dual purpose; for example, ethyl and methyl centralite function as both stabilizers and plasticizers.

In addition to the energetic compounds, the primer also contains metal compounds that contribute to the formation of the spherical oxide and sulfide particulates. The review article by Dalby contains an extensive, but not exhaustive, list of inorganic compounds that may contribute to GSR. ${ }^{10}$ Compounds such as antimony sulfide and sulfite, barium nitrate and peroxide, and lead dioxide and peroxide are included. The compounds and elements of interest in this research are found in Table 1.1. 
Table 1.1 Target compounds and elements and their sources along with their location within this text.

\begin{tabular}{|l|l|l|}
\hline \multicolumn{1}{|c|}{ TARGET } & \multicolumn{1}{c|}{ SOURCE } & \multicolumn{1}{c|}{$\begin{array}{c}\text { LOCATION IN } \\
\text { TEXT }\end{array}$} \\
\hline Ethyl Centralite (EC) & Propellant powder - stabilizer/plasticizer & Chapter 2 \& 3 \\
\hline Methyl Centralite (MC) & Propellant powder - stabilizer/plasticizer & Chapter 2 \& 3 \\
\hline Diphenylamine (DPA) & Propellant powder - stabilizer & Chapter 2 \& 3 \\
\hline $\begin{array}{l}\text { Dimethyl Phthalate } \\
\text { (DMT/DMP) }\end{array}$ & Propellant powder - plasticizer & Chapter 2 \& 3 \\
\hline 2,4-Dinitrotoluene (2,4-DNT) & Propellant powder - flash suppressor & Chapter 3 \\
\hline Carbazole (Carb) & Propellant powder - stabilizer & Chapter 3 \\
\hline Dibutyl Phthalate (DBP) & Propellant powder - plasticizer & Chapter 3 \\
\hline $\begin{array}{l}\text { 2-Nitrodiphenylamine (2- } \\
\text { NDPA) }\end{array}$ & Propellant powder - stabilizer & Chapter 3 \\
\hline $\begin{array}{l}\text { 4-Nitrodiphenylamine (4- } \\
\text { NDPA) }\end{array}$ & Propellant powder - stabilizer & Chapter 3 \\
\hline Lead & Primer & Chapter 4 \\
\hline Barium & Primer & Chapter 4 \\
\hline Antimony & Primer & Chapter 4 \\
\hline Copper & Primer & Chapter 4 \\
\hline
\end{tabular}

\subsubsection{Sampling}

There are various techniques used to sample for FDR including adhesive tapes, glues, swabbing, and vacuum lifts. Adhesive tapes or lifters are the most common and are used for collecting mainly inorganic residues. Typically a carbon-containing adhesive is located on the end of an aluminum stub with one of two surface areas, $126.7 \mathrm{~mm}^{2}$ or $506.7 \mathrm{~mm}^{2}$. This type of collection eliminates direct contact with the collecting officer and the sampling surface. ${ }^{12}$ The conductive carbon coating on the adhesive prevents charging when analyzed with scanning electron microscopy (SEM) discussed below. The second most common FDR sampling technique and the one used in the presented research, is swabbing which is used to collect both 
inorganic and organic residues. ${ }^{13}$ Typically a fiber substrate (swab) is soaked with a solvent, aqueous or organic, and swiped across the tops and palm sides of the hands. Many studies have been performed testing and comparing the efficiency of adhesives and tape lifts to other methods ${ }^{3,14-16}$ and testing solvents, substrates, and extraction methods for swabbing. ${ }^{17-19}$ Review articles by Dalby et al..$^{10}$ and Romolo et al. ${ }^{20}$ summarize the results of these studies.

\subsubsection{Current Forensic Analytical Methodology}

Colorimetric tests, also referred to as color or spot tests, were used in previous years for GSR presumptive testing in the field. These types of tests target specific compounds and functional groups in which the change in reagent color or appearance of a color is indicative of a chemical reaction taking place. Color tests have been used for testing for the presence of GSR, determination of bullet holes and entrance wounds, and most commonly estimating firing distance. ${ }^{10}$ Common GSR color tests include the dermal nitrate/paraffin test, sodium rhodizonate test (lead and barium), and Griess test (nitrites). The disadvantage of GSR color tests is the non-specific nature of the targets and their common environmental occurrences. Contamination from sources other than GSR such as nitrites from tobacco, fertilizers, and urine, produce a false positive for GSR. ${ }^{10,20}$ For this reason, color tests are rarely used today with the exception of their use in distance determination.

Current confirmatory analysis for FDR is scanning electron microscopy/energy dispersive x-ray spectrometry (SEM-EDS) and has been used in casework since the late 1970's. ${ }^{4}$ SEM-EDS is a non-destructive method that detects FDR based on the morphological and elemental composition of the inorganic particulates (GSR). A standard method (ASTM 1588) specifies the procedure and classification for gunshot residues analyzed via SEM-EDS. ${ }^{21}$ It defines the morphology as being spheroidal, non-crystalline and typically between 0.5-5.0 $\mu \mathrm{m}$ in 
diameter. The standard classifies particulates based on elemental composition and classifies them as being "characteristic of" or "consistent with" GSR based on the elements identified. To be characteristic of GSR a particle must contain lead, antimony, and barium; to be consistent with the particle can contain a combination of 1 or 2 of these elements along with containing calcium, potassium, silicon, aluminum, phosphorus, chlorine, sulfur, iron, nickel, copper, zinc, zirconium, and/or tin. ASTM 1588 has also classified the composition of particles from ammunition with lead free/non-toxic primers. Gadolinium, titanium, zinc or gallium, copper, tin are elemental compositions characteristic of GSR particles from lead free ammunition (LFA). A consistent GSR particle elemental composition includes titanium, zinc or any of the following additional elements: aluminum, silicon, calcium, copper, or tin. Strontium is also included as being observed in LFA.

\subsection{Purpose}

FDR evidence is being utilized less frequently than in past years for several reasons. Currently, there are few if any reliable and viable field/presumptive test methods available resulting in the inability to filter samples for laboratory analysis. SEM instrumentation is relatively expensive and, because it is not routinely used for additional forensic evidence, it is not likely to be purchased by cash-poor forensic laboratories. Additionally, traditional confirmatory forensic analysis methods of FDR detection focus on the inorganic particulates (GSR) originating from compounds found in the primer of the cartridge. While the SEM-EDS method is technically sound and effective, limitations with the target particulates exist. For example, particulate GSR is subject to secondary transfer which can complicate the interpretation of findings. Furthermore, SEM-EDS methods target only a fraction of the discharge residue leaving potentially valuable portions unexamined. 
Although recent interest has spurred the development and/or advancement of a technique for analyzing OGSR, to date, such methods have not been implemented in forensic laboratories. Figure 1.1 reviews the progression of FDR research in our laboratory beginning with a preliminary study on persistence of OGSR on the hands of known shooters with ion mobility spectrometry (IMS) in 2011. ${ }^{7}$ Results indicated that OGSR persisted for at least 4 hours and the lipophilic nature of the OGSR compounds likely prevented secondary transfer. This implied that loss of OGSR was likely due to absorption or evaporation if not being deliberately removed by hand washing. Initial permeation studies with Franz diffusion cells and IMS supported the implication that OGSR compounds are dermally absorbed. ${ }^{9}$ The rate of absorption is compound dependent and correlates with the 3-4 hour persistence previously reported. Additional permeation studies performed with GC/MS provided deposition amounts for 5 OGSR compounds and implications for skin sampling and analysis. ${ }^{2}$ By combining experimental and modeling data, a window of detectability was estimated for three OGSR compounds with three analytical instruments; IMS, GC/MS, and LC/MS/MS. It was concluded that OGSR may be detectable up to nearly 24 hours given the right sampling and analytical procedures.

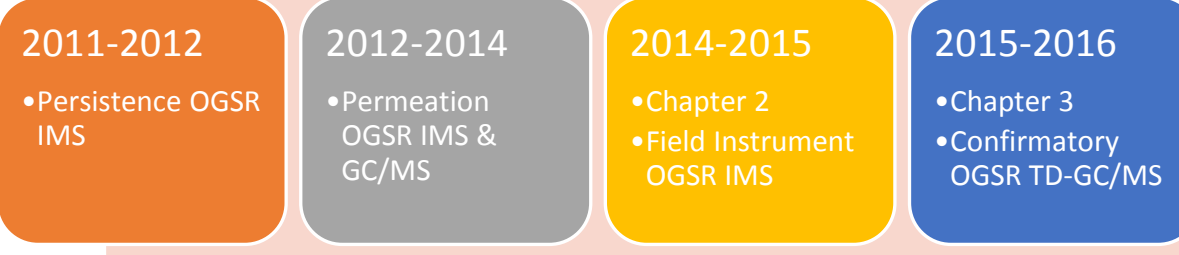

\section{6}

- Chapter 4

-H-G GSR

ESI/MS/MS

Figure 1.1: Timeline of OGSR research performed in our laboratory. 
These studies have paved the foundation for the research presented here. Currently, there is not a reliable rapid presumptive test for FDR. In both studies IMS proved to be fit for purpose and valuable for detecting OGSR and it also showed promise as a FDR field screening instrument. The goal of the research presented in Chapter 2 was to evaluate the potential use of ion mobility spectrometry as a means for screening for OGSR on hand swabs. Presumptive tests provide a means of screening samples prior to being sent to forensic laboratories for testing. As backlogs are continuously growing in forensic laboratories it is important to provide a means of screening samples prior to resources (time, money, etc.) being wasted on analysis.

In addition to developing and validating presumptive tests, confirmatory test methods also need researched and developed. Confirmatory instruments, such as GC/MS and LC/MS ${ }^{\mathrm{n}}$, are currently already available in most forensic laboratories. Developing methods for these instruments would keep costs low as a new instrument would not need purchased and personnel would not need to be trained on a new instrument, making the implementation of new detection methods into this field easier. The remaining research chapters, Chapters $3 \& 4$, utilize these confirmatory instruments in novel ways for FDR detection.

The goal of the research in Chapter 3 was to evaluate thermal desorption as a sample introduction method for gas chromatography mass spectrometry, also for the analysis of OGSR hand swabs. In published work from our laboratory and work presented here (Chapter 2) thermal desorption proved efficient for sample introduction for IMS detection. Thermal desorption allows volatile organic compounds to be extracted from sampling media without any sample preparation or pre-treatment and coupling with GC/MS allows for compound confirmation. GC/MS also allows for the detection of multiple compounds unlike IMS. 
While detecting multiple OGSR compounds is an advantage over traditional GSR detection methods (SEM/EDS), GSR analysis must not be eliminated, as new methods are developed for OGSR detection. Ideally, a method would utilize propellant residues both particles and particulates and organic condensates as a means of detection and identification of firearms discharge residue. This would provide a wealth of evidentiary information and increase probative value. Chapter 4 focuses on the exploration of detecting GSR with ESI tandem mass spectrometry through complexing with supramolecular compounds such as crown ethers. While ESI/MS ${ }^{\mathrm{n}}$ is typically reserved for the analysis of organic compounds, complexation allows for detection of metal ions overcoming issues previously experienced with elemental ESI/MS ${ }^{\mathrm{n}}{ }^{22}$ This research was done in an effort to provide a means in which OGSR and GSR can be simultaneously detected. The overall goal of the research presented here was to address some of the limitations with current methodology and in doing so increase the evidentiary value of FDR evidence.

\subsection{Instrumentation}

\subsubsection{Ion Mobility Spectrometry (IMS)}

Ion mobility spectrometry (IMS) characterizes substances based on the mobility of gas phase ions in an electric field. An ion mobility spectrometer (Figure 1.2) is comprised of two main regions; an ionization or reaction region and a drift region. Ionization of a volatilized sample occurs in the ionization region commonly in air at ambient pressure through gas-phase reactions with reactant ions formed through beta emitters, such as the radioactive isotope ${ }^{63} \mathrm{Ni}^{23}$ A shutter grid, located just prior to the drift region, gates the ions resulting in the formation of an ion packet or swarm. Once the potential on the grid is dropped, the ion swarm enters the drift region and ions move toward the detector via a potential gradient created by applying increased 
potential to drift rings. Within the drift region, analyte ions collide with inert buffer/drift gas (i.e. He) flowing countercurrent to the ions and the ions are separated based on their mobilities through the gas.

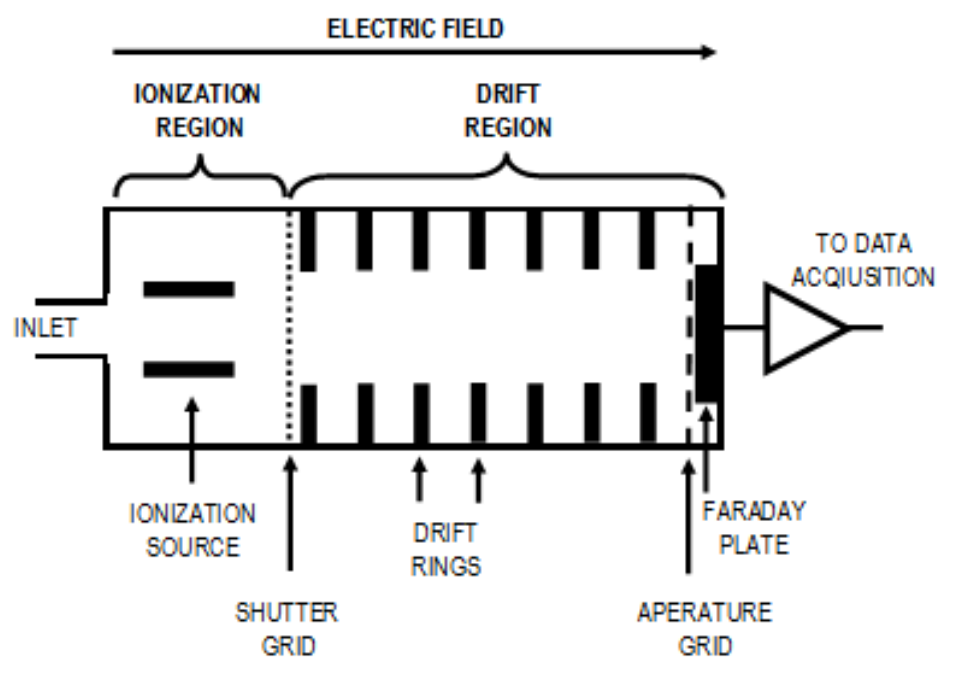

Figure 1.2 Schematic of an ion mobility spectrometer.

Ion mobility $\left(\mathrm{K}, \mathrm{cm}^{2} \mathrm{~V}^{-1} \mathrm{~s}^{-1}\right)$ is defined by the drift velocity $\left(\mathrm{v}_{\mathrm{d}}, \mathrm{cm}^{2} \mathrm{~s}^{-1}\right)$ of an ion divided by the electric field $\left(\mathrm{E}, \mathrm{V} \mathrm{cm}^{-1}\right)$ (Equation 1.4.1) $)^{23}$ :

$$
K=v_{d} / E
$$

where velocity is determined by the time is takes for an ion or ion swarm to travel the distance $(\mathrm{d}, \mathrm{cm})$ between the shutter grid and detector also known as drift time $\left(\mathrm{t}_{\mathrm{D}}, \mathrm{s}\right)\left(\right.$ Equation 1.4.2) ${ }^{23}$ :

$$
v_{d}=d / t_{D}
$$

Mobility, K, is commonly normalized to standard temperature (273 K) and pressure (760 torr) due to the effects buffer gas temperature $(\mathrm{T})$ and pressure $(\mathrm{P})$ have on drift velocity, resulting in what is known as reduced mobility coefficient $\left(\mathrm{K}_{\mathrm{o}}\right)$ (Equation 1.4.3) ${ }^{23}$ :

$$
K_{o}=K(273 / T)(P / 760)
$$


The normalization of mobility allows for the comparison of measurements performed on different instruments under different experimental conditions.

Two IMS instruments, a benchtop and a handheld, were utilized in these studies (Chapter 2) and were evaluated as a means for screening hand swabs for OGSR. IMS instruments are routinely used in security and military applications for the detection of explosives, narcotics, and chemical warfare agents. ${ }^{23-25}$ Their ability to operate at atmospheric conditions, and be lightweight and easy to use, make IMS devices ideal instruments for these types of applications. Typically samples are introduced into these handheld instruments by collecting particles on swabs by swabbing or by sniffing the air. Alarms for compounds of interest can be programmed into the device and sound when a peak is present in the specified drift time window. Although IMS instruments are rugged and generally reliable, drift times are not unique to a given compound resulting in the instrument being subject to false positives. Therefore, IMS instruments should be used for screening purposes and care should be taken when interpreting IMS spectra.

\subsubsection{Gas Chromatography - Mass Spectrometry (GC/MS)}

Gas chromatography mass spectrometry is a staple instrument in forensic science laboratories with applications in arson investigations, drug analysis, and more. The basic components of a gas chromatograph (Figure 1.3) are a carrier gas, sample injection port, and a capillary column housed in an oven. A sample is injected/inserted into the GC via the injection port. The GC utilized in these studies was equipped with a commercially available specialized injection port fitting called a thermal separation probe (TSP) that employs thermal desorption as the means for sample introduction. Further discussion and more information on the TSP is provided in Chapter 3. 


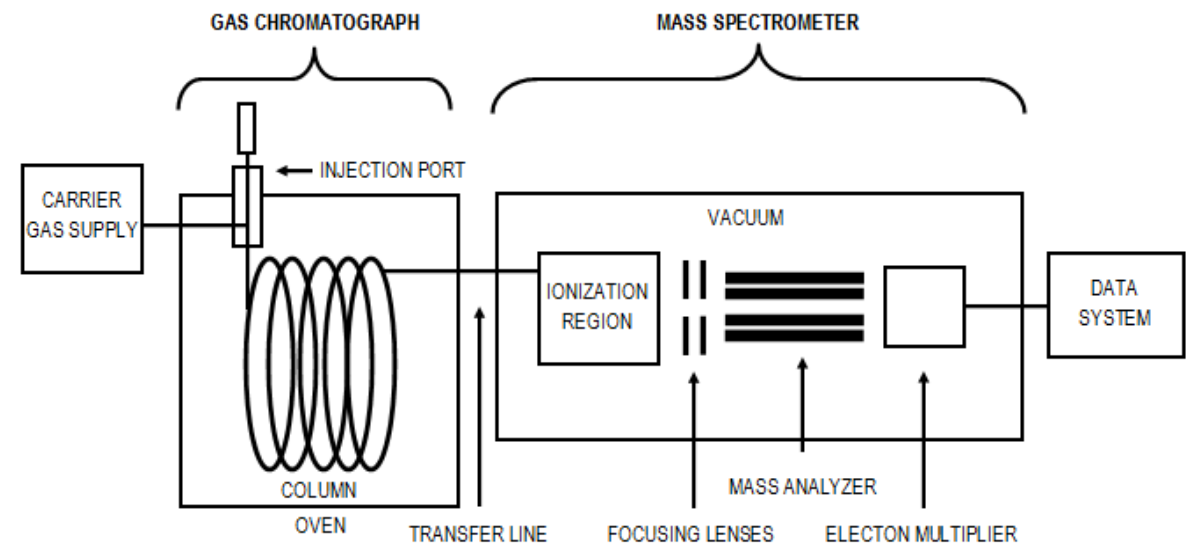

Figure 1.3 Schematic of a GC/MS system.

Heat from the port volatilizes the sample and a carrier gas passes the volatile and semivolatile analytes onto the column. As the analytes are swept through the column separation occurs as a result of the analytes partitioning between the mobile and stationary phases. The mobile phase or carrier gas is a high purity inert gas most commonly helium, although argon, nitrogen, and hydrogen are sometimes used. ${ }^{26}$ The stationary phase, commonly a polysiloxane derivative, is coated onto the inner walls of the polyimide coated fused silica glass capillary and ranges in thickness from 0.5 to 5 microns. The oven, housing the capillary column, is temperature controlled and by using a temperature program (i.e. ramping the temperature) separation is facilitated and analysis time reduced. Low temperatures allow for more volatile analytes to resolve whereas increased temperatures resolve lower or semi-volatile analytes.

Analytes eluting from the column are directed into the mass spectrometer (Figure 1.3) where they are ionized, analyzed, and detected. Electron impact ionization, one of the most common ion sources utilized by GC/MS, ionizes the gaseous analytes by way of electron bombardment $(70 \mathrm{eV})$. Interactions with high-energy electrons cause target molecules to lose electrons and produce a molecular ion and further fragmentation occurs as the molecules relax 
from highly excited vibrational and rotational states. By applying a small potential difference on an extraction plate, ions are extracted towards an Einzel lens stack where they are focused into Q of the quadrupole assembly.

A quadrupole mass analyzer is the most common type of mass analyzer ${ }^{26}$ and the one contained in the GC/MS utilized in these studies (Chapter 3). A quadrupole is comprised of four parallel cylindrical rods with dc and ac (RF) voltages applied to each rod. As ions are accelerated through the center of the rods, the dc and ac voltages are increased simultaneously. The stability of the ions trajectory through this oscillating electric field results in the ions being separated according to their mass-to-charge $(\mathrm{m} / \mathrm{z})$ ratio. As the ions exit the quadrupole, a detector, typically an electron multiplier, amplifies the separated ions and converts the ions into an electrical signal. The MS components are held under high vacuum (low pressure) to provide the generated ions with a free path, eliminating gaseous molecule that the ions could undergo collisions with as they travel to the detector. The resulting products are a chromatogram of retention time, the time at which the analytes come off of the column, versus signal intensity and a mass spectrum of $m / z$ versus abundance.

\subsubsection{Electrospray Ionization Tandem Mass Spectrometry (ESI-MS/MS)}

Tandem mass spectrometers can be classified into two categories; tandem-in-space or tandem-in-time. The instrument utilized in this research (Chapter 4), the triple quadrupole mass spectrometer, is the most common tandem-in-space instrument. ${ }^{26}$ The basic components of a triple quadrupole mass spectrometer instrument, depicted in Figure 1.4, are an ion source, three quadrupoles, and a detector. 


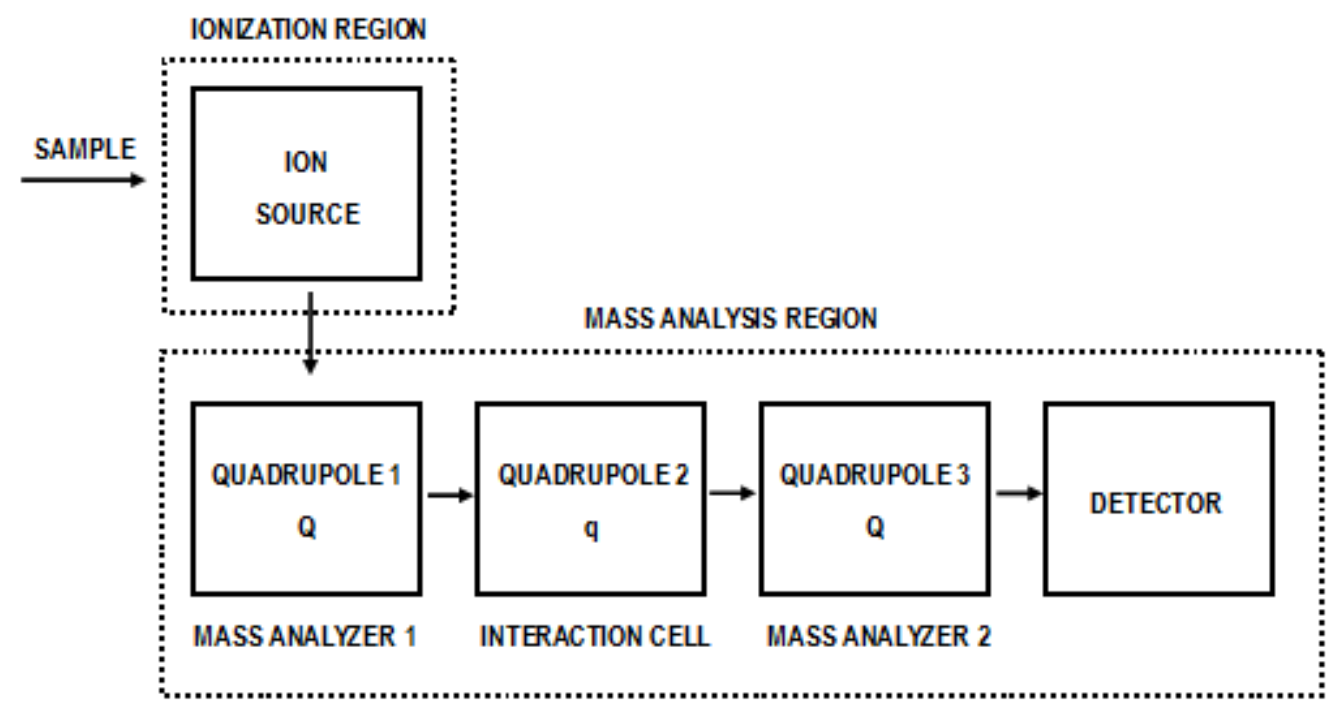

Figure 1.4 Block diagram of a triple quadrupole mass spectrometer.

The ion source in the instrument utilized employs electrospray ionization (ESI) which is a soft atmospheric pressure ionization technique in which ions can be transferred from solution into the gas phase. ESI is capable of ionizing large, non-volatile, chargeable molecules with molecular weights of 100,000 Da or more. ${ }^{26,27}$ The softness of this technique, a result of differential pumping from atmospheric pressure to high vacuum, allows for the analysis of native state proteins or in the interest of this research, host-guest molecules. ${ }^{27}$

Solution at a flow rate of $1-20 \mu \mathrm{L} \mathrm{min}{ }^{-1}$ enters into the stainless steel capillary held at a high potential of 3-4 kV. Upon exiting the capillary the emerging liquid is under the influence of an electric field, causing charge separation. At the voltage (onset voltage) in which pressure overcomes surface tension, a Taylor cone is formed and a fine jet of charged liquid emerges flowing in the direction of the counter electrode. ${ }^{27,28}$ Droplets are produced from the jet stream as a result of instability caused by the high charge density. ${ }^{27} \mathrm{~A}$ heated transfer capillary or curtain gas drives solvent evaporation. As solvent continuously evaporates, the electric field of the droplet increases as a result of the decreasing radius causing the droplets to undergo 
Coulomb fission repeatedly until ion formation. There are two models used to describe the formation of ions from charged droplets; charged-residue model (CRM) and ion evaporation model (IEM). The CRM describes the formation of ions through solvent evaporation and declustering. Conversely, the IEM model, describes the formation of ions through desorption from the droplet surface.

Once formed, the ions flow through the orifice of the curtain plate into quadrupole $1(\mathrm{Q})$ for the first stage of mass separation. The quadrupole method of separation is described previously in Section 1.3.2. It is here, in the first mass analyzer, that precursor ions are selected. The precursor ions then enter quadrupole $2(\mathrm{q})$, the collision cell, where ions interact with collision gas to produce product ions. This process is called collision induced dissociation (CID) occurs and the resulting product ions then pass into the third and final quadrupole (Q) for the second stage of mass separation. The ions are then detected and a tandem mass spectrum is produced. The type of mass spectrum produced is determined by the scanning mode utilized.

\subsection{Certificates and Training}

The research performed consisted of human subject sampling in the form of swabbing of the hands with a swab made of muslin or a similar material. Prior to the commencement of this research West Virginia University's Institutional Review Board (IRB) approval was received for the human subject sampling (WVU IRB protocol \#1209000337). West Virginia University training courses provided by the Collaborative Institutional Training Initiative (CITI) Program were also completed, including Conflicts of Interest and Biomedical Research Investigators. Additionally, a majority of this research required discharging a firearm for the collection of the hand swabs. A certificate for the National Rifle Association (NRA) concealed carry gun training 
was acquired to learn proper firearm handling and safety precautions to ensure that the utmost safety during the handling and discharging of a firearm. 


\subsection{References}

1. G. Wolten and R. Nesbitt, 1980.

2. J. W. Moran and S. Bell, Analytical Chemistry, 2014, 86, 6071-6079.

3. D. K. Shaffer and K. Yi, Scanning, 1999, 21, 99-100.

4. B. J. Heard, Forensic Ballistics in Court : Interpretation and Presentation of Firearms Evidence, John Wiley \& Sons, Somerset, NJ, USA, 2013.

5. T. Jalanti, P. Henchoz, A. Gallusser and M. S. Bonfanti, Sci. Justice, 1999, 39, 48-52.

6. Z. Brozek-Mucha, Microsc. microanal., 2011, 17, 972-982.

7. J. Arndt, S. Bell, L. Crookshanks, M. Lovejoy, C. Oleska, T. Tulley and D. Wolfe, Forensic Sci.Int., 2012, 222, 137-145.

8. M. X. Zhao, S. C. Zhang, C. D. Yang, Y. C. Xu, Y. X. Wen, L. S. Sun and X. R. Zhang, J. Forensic Sci., 2008, 53, 807-811.

9. J. Moran and S. Bell, Int. J. Ion Mobil. Spec., 2013, 16, 247-258.

10. O. Dalby, D. Butler and J. W. Birkett, J. Forensic Sci., 2010, 55, 924-943.

11. R. V. Taudte, A. Beavis, L. Blanes, N. Cole, P. Doble and C. Roux, BioMed research international, 2014, 2014, 965403-965403.

12. M. Tassa, N. Adan, N. Zeldes and Y. Leist, J. Forensic Sci., 1982, 27, 671-676.

13. J. S. Wallace and W. J. McKeown, Journal of the Forensic Science Society, 1993, 33, 107-116.

14. H. A. Wrobel, J. J. Millar and M. Kijek, J. Forensic Sci., 1998, 43, 178-181.

15. D. Degaetano, J. A. Siegel and K. L. Klomparens, J. Forensic Sci., 1992, 37, 281-300.

16. A. Zeichner and N. Levin, J. Forensic Sci., 1993, 38, 571-584.

17. J. D. Twibell, J. M. Home, K. W. Smalldon, D. G. Higgs and T. S. Hayes, J. Forensic Sci., 1982, 27, 792-800.

18. J. B. F. Lloyd and R. M. King, J. Forensic Sci., 1990, 35, 956-959.

19. M. R. Reardon and W. A. MacCrehan, J. Forensic Sci., 2001, 46, 802-807.

20. F. S. Romolo and P. Margot, Forensic Sci.Int., 2001, 119, 195-211. 
21. ASTM-International, ASTM E1588-10e1: Standard Guide for Gunshot Residue Analysis by Scanning Electron Microscopy/ Energy Dispersive X-ray Spectrometry, https://www.astm.org/cgi-bin/resolver.cgi?E1588-16a).

22. W. Z. Shou and R. F. Browner, Analytical Chemistry, 1999, 71, 3365-3373.

23. G. A. Eiceman, Z. Karpas and H. H. Hill, Ion Mobility Spectrometry, Third Edition, CRC Press, 2013.

24. G. A. Eiceman and J. A. Stone, Analytical Chemistry, 2004, 76, 390A-397A.

25. R. G. Ewing, D. A. Atkinson, G. A. Eiceman and G. J. Ewing, Talanta, 2001, 54, 515529.

26. J. H. Douglas Skoog, Stanley Crouch, Principles of Instrumental Analysis, Brooks/Cole, Belmont, CA, Sixth edn., 2007.

27. J. H. Gross and P. Roepstorff, Mass Spectrometry: A Textbook, Springer Berlin Heidelberg, 2011.

28. E. de Hoffmann and V. Stroobant, Mass Spectrometry: Principles and Applications, Wiley, 2007. 


\section{Chapter 2: Evaluation and validation of Ion Mobility Spectrometry for Presumptive Testing Targeting the Organic Constituents of Firearms Discharge Residue ${ }^{1}$}

${ }^{1}$ Reproduced from the Royal Society of Chemistry's Analytical Methods journal: Evaluation and validation of ion mobility spectrometry for presumptive testing targeting the organic constituents of firearms discharge residue. B.Yeager, K. Bustin, J. Stewart, R. Dross and S. Bell, Analytical Methods 7, 9683-9691, 2015. DOI 10.1039/C5AY02417J http://pubs.rsc.org/en/content/articlelanding/2015/ ay/c5ay02417j\#!divAbstract

Permission was obtained from the co-authors and was not required by the journal according to the Royal Society of Chemistry's licenses, copyright, and permissions policy. The policy is located at the following web address: http://www.rsc.org/journals-books-databases/journal-authors-reviewers/licencescopyright-permissions

Firearms discharge residue (FDR) refers to both the inorganic particulates (GSR) and the organic constituents (OGSR) formed when a firearm is discharged. Traditional methods are based on the detection of the inorganic particulates which are formed from the metals in the primer.

Currently, there are few if any viable presumptive or screening tests amenable to detection of FDR on skin. Ion mobility spectrometry (IMS) is already widely deployed in law enforcement and homeland security for use as a portable/presumptive detector for narcotics and explosives. In addition, most commercial instruments can detect several organic constituents commonly found in FDR without requiring modification of instrumentation. The goal of this project was to evaluate IMS for use as a screening device to detect OGSR on hand swabs. Two instruments were thoroughly tested and figures or merit, including detection thresholds established. Sample stability was also characterized with significant degradation seen when samples were stored at room temperature. Results showed that given proper and specialized QA/QC procedures, IMS can be successfully utilized for screening purposes. Analysis of more than 200 skin swab samples demonstrated that pattern matching data analysis is preferred to peak-based methods when attempting to ascertain if a person recently fired a weapon. 


\subsection{Introduction}

When a firearm is discharged, a firing pin strikes and activates a shock sensitive primer which then ignited the gunpowder contained within the cartridge. Heat and pressure vaporize the metals from the primer and the gaseous product of the burning propellant causes an increase in pressure and the projectile to be expelled from the cartridge. As the bullet travels the length of the barrel and is expelled, vapors and particles escape from the weapon. This radial expulsion, known as a plume, is a complex heterogeneous mixture of compounds comprised of inorganic particulates (GSR), particles of un-burnt and partially burnt propellant, and organic condensates (OGSR collectively). During a deposition event, this complex mixture of compounds falls on surrounding surfaces including the hands, chest, shoulders, and face of the person discharging the weapon. The amount of residue deposited varies depending on the ammunition and weapon used and the environmental conditions during the firearm discharge event (Figure 2.1).

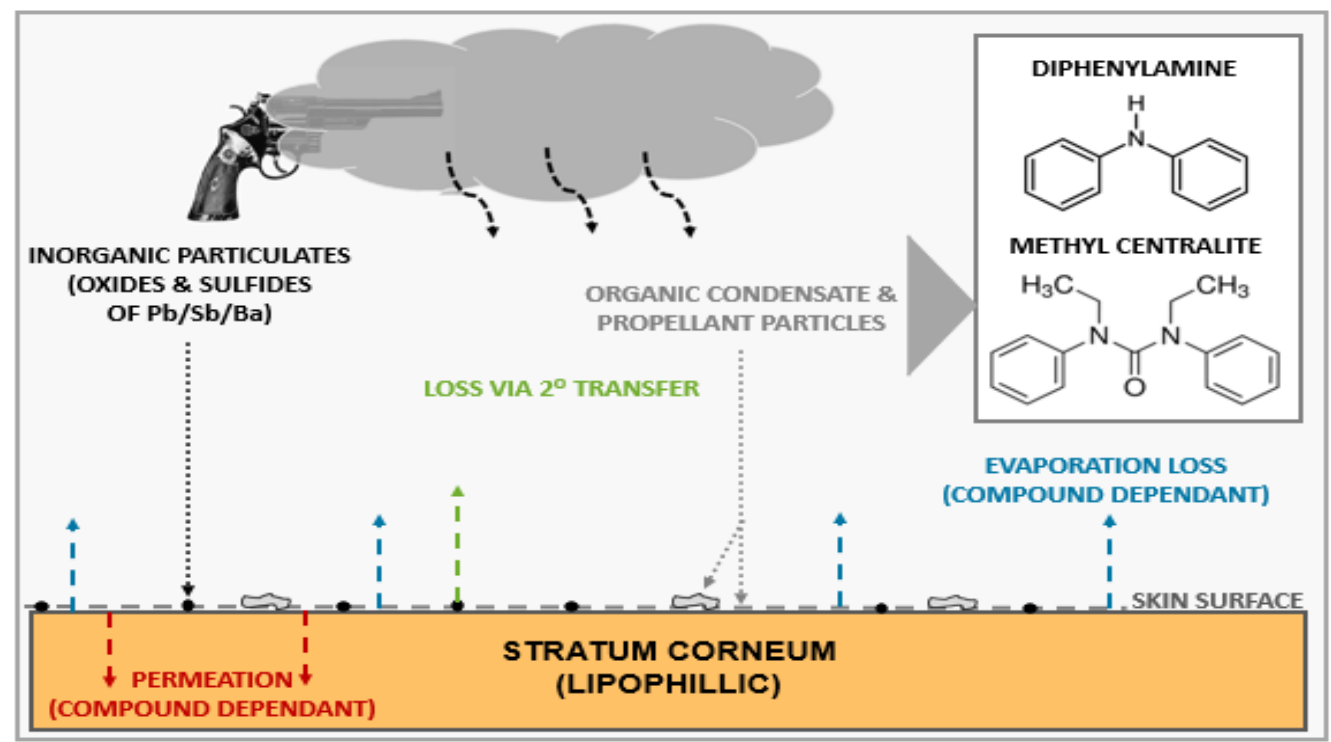

Figure 2.1 Physical and chemical evidence produced by a firearm discharge. 
Traditional analytical techniques applied to GSR such as scanning electron microscopy energy dispersive $\mathrm{x}$-ray spectroscopy (SEM/EDS) focus on detecting the inorganic particulates (GSR) formed during the flash heating and condensation of compounds in the primer. The volatilized metals condense as spherical oxides or sulfides to form smooth particulates anywhere from $\sim 0.5$ to 5.0 microns in size. Particulate GSR evidence has a limited persistence of approximately 4 hours ${ }^{1-3}$ and can be transferred through a simple handshake and easily removed through minor physical activity or hand washing.

OGSR compounds are generally ancillary compounds such as stabilizers rather than the energetics. $^{4-9}$ Some of the most commonly studied OGSR compounds are ethyl centralite (EC), methyl centralite (MC), dimethyl phthalate (DMT), diphenylamine (DPA) and its nitration products, n-nitrosodiphenylamine (N-NODPA), and 2-, and 4-nitrodiphenylamine (2NDPA and 4NDPA). ${ }^{5,6,9-11}$ Nitroglycerin can also be present but was not targeted in this study due to expected instability in stored samples. Additionally, positive mode ions were targeted rather than negative mode due to instrument configuration which limited the analysis to one mode at a time which meant that the sample was irreversibly compromised with the first analytical cycle.

There are compelling reasons to develop methodology targeting OGSR as a screening assay. IMS is well-suited for detection of related compounds such as energetics and has been used as such for years. Color tests such as the Griess test for nitrates/nitrites suffer from the ubiquitous nature of these anions in the environment. Other color tests are similarly limited by high false positive/false negative findings and the destructive nature of the testing. Because OGSR is found in larger amounts compared to primer residues, presumptive assays that target the organic constituents would be expected to have greater utility compared to those targeting primer residues. As manufactures' move away from heavy metals in primers, the utility of 
SEM/EDS methods will inevitably be impacted. Finally, given current advances in mass spectrometry (MS) including MS methods available to forensic laboratories, there is no reason not to develop analytical schemes targeting OGSR, from presumptive assays through confirmatory analysis. IMS could fill a role as a field testing or laboratory screening device that would be useful in selecting samples best suited for costly and time-consuming confirmatory analysis, be that with SEM/EDS or mass spectrometry.

Ion mobility spectrometry has been routinely used for detection of explosives, narcotics and chemical warfare agents in security and military applications since the 1980 's. ${ }^{12-15}$ The most common applications are for detecting chemical warfare agents, narcotics, and explosives/energetics. The instruments evaluated here operate at atmospheric pressure and utilize a soft ionization technique to generate ion/molecule clusters that are characterized by their drift times in a low electric field. The portability and proven ruggedness of IMS instruments make them well-suited to forensic screening applications given proper analytical methods and proper interpretation. As an added advantage, IMS can also detect multiple peaks and patterns that are amenable to chemometric and statistical analysis which could be developed into a probabilistic model. The output of such an analysis would be phrased in terms of a probability of recently firing a weapon rather than an alarm/no alarm signal. This is the role envisioned for IMS and similar field screening instrument. However, the ability to deploy an instrument in this capacity requires intensive development from method and procedural validation (the focus of this report) through population studies and chemometric evaluation. The motivation for undertaking this work is that far more information than can be obtained from a mobility spectrum than a simple color test; the more information that is collected, the lower the anticipated rates of false 
positives/false negatives and the more effective the entire analytic process. Finally, IMS is ideally suited for use with swabs collected from the skin which was the matrix of interest here.

This report will focus on characterization of four representative and commonly studied OGSR compounds (Table 2.1). These compounds were selected based on their presence in detectable quantities in authentic firearm discharge samples ${ }^{9,10}$ and apparent resistance to secondary transfer which may in part be attributed to the lipophilic nature of the organic condensates (Figure 2.1). Particles of unburnt and partially burnt propellant would however be expected to be subject to secondary transfer. ${ }^{6}$ Time studies of some common OGSR compounds on the hands of shooters have been previously studied using IMS with persistence of several hours noted. ${ }^{6,11}$ The goals of this project were to (1) optimize IMS instruments for OGSR using diphenylamine; (2) validate instruments and generate figures of merit; and (3) demonstrate IMS is fit-for-purpose as a presumptive testing method for OGSR.

Table 2.1 Target compounds and associated material. $\mathrm{Vp}$ is vapor pressure in $\mathrm{mm} \mathrm{Hg}$ at $25^{\circ} \mathrm{C}$ with sources noted below.

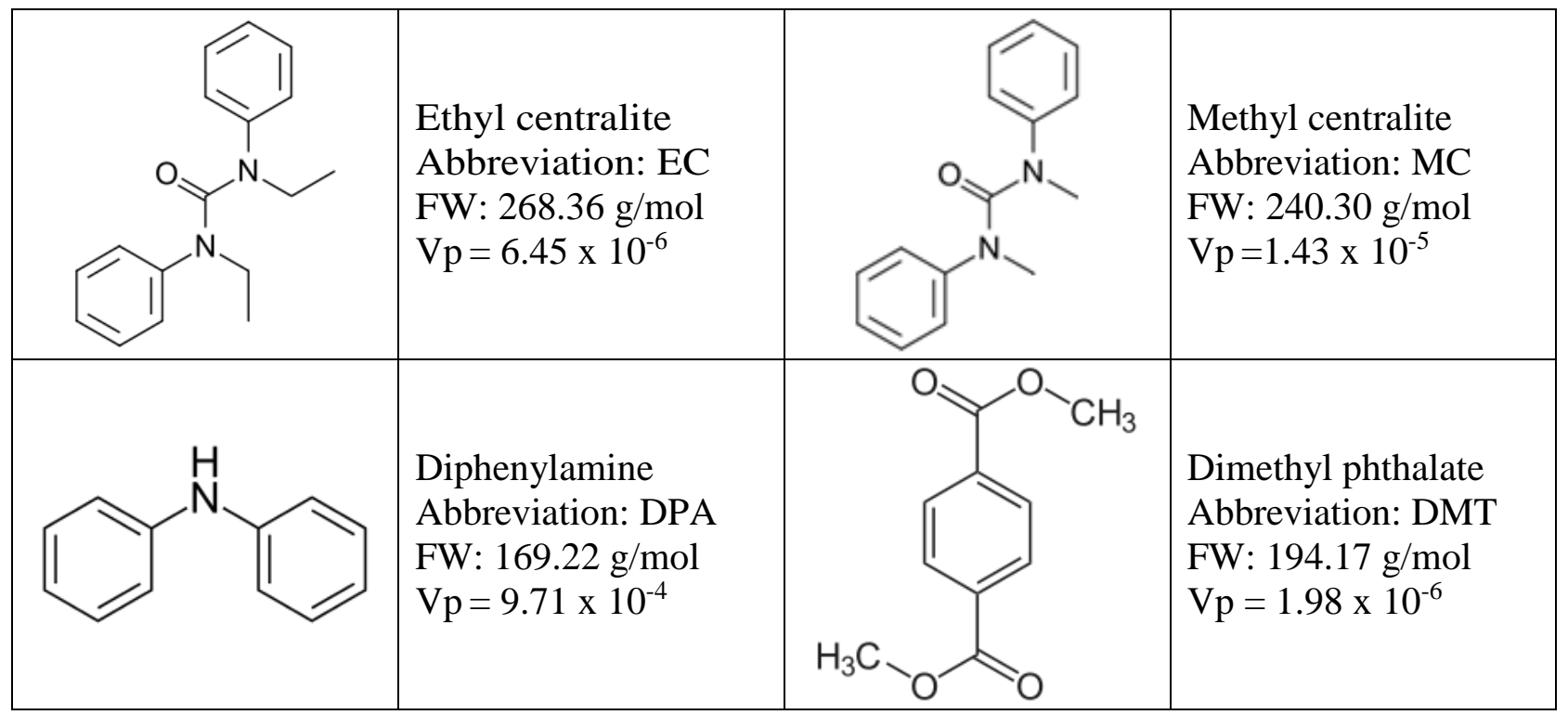

For comparative purposes, all vapor pressures are from Chemspider (www.chemspider.com), predicted, US Environmental Protection Agency's EPISuite ${ }^{\mathrm{TM}}$. 
Two commercially available IMS instruments were utilized; a handheld model, Sabre $4000 \AA$, and a benchtop model, Ionscan-LS®. These instruments share the same basic drift tube design and software packages. Both utilize thermal desorption for sample introduction but with slightly different implementations. Samples are introduced into the Sabre 4000® through a slit causing the desorber to automatically actuate and press against the swab and directing flow over the portion of the swab surface in contact with the desorber. With the Ionscan-LS®, samples are first cut and placed on a Teflon ${ }^{\circledR}$ membrane before being slid into the desorption region where the desorber presses the sample into position. The two instruments were purchased in the late 2000's and were used for different purposes over the years before being used in this project. The use of the two instruments by different analysts over time provides reasonable and realistic quantitative descriptors of repeatability and reproducibility for this application. However, the designs precluded the analysis of positive and negative mode ions simultaneously. Consequently, the results of this study reflect a baseline capability as opposed to the best possible performance.

\subsection{Materials and Methods}

\subsubsection{Sample Preparation \& Analysis}

Standard solutions of ethyl centralite (EC), methyl centralite (MC), dimethyl phthalate (DMT) and diphenylamine (DPA) at concentrations of 100 ppm, 100 ppm, 1000 ppm, and 5000 ppm respectively were obtained from AccuStandard (New Haven, CT USA). Standard solutions were stored at temperatures recommended by the manufacturer and once opened they were transferred to $1 \mathrm{~mL}$ clear glass vials.

Media was selected based on the following factors: wettability, instruments compatibility, vender-neutrality, commercial availability, and background interference. Wettability is important for sampling collection efficiency, thus the selected media must be compatible with 
benign solvents such as isopropanol or ethanol. Vendor-neutrality was also a factor in that the media should be amenable to use in a variety of instrument makes and models. Based on this criterion, a muslin swab from DSA Detection (Boston, MA) was selected as the sampling media. Muslin is commonly used as a wiping substrate for portable instruments like ion mobility spectrometers.

Samples to be used for the validation study were prepared by spiking $1.0 \mu \mathrm{L}$, with a syringe, of the standards at stock or dilute concentrations onto muslin swabs. Swabs, if not used immediately, were then placed spiked side up in a plastic petri dish obtained from Falcon (Corning, NY) before being taped shut for the duration of the study.

Drift times for each compound were established using commercial reference standards. Drift times were used in this study rather than reduced mobilities because both instruments have an internal calibrant (nicotinamide) to which drift times are automatically adjusted and the target compounds were known and could be unambiguously assigned to a mobility peak. Background samples of laboratory air were analyzed at the beginning and completion of analysis and between samples to determine the presence of contamination or sample carry over. If the resulting spectra show signs of either a short bake out cycle was performed and if persistent no sample analysis was conducted until contamination or carry over was removed. Blank media background samples were also obtained on a daily basis.

Mobility spectra were collected upon actuation of the desorber and collected continuously for 20 seconds with the spectral pattern evolving across the desorption profile. Analysts viewed all collected spectra in the desorption profile for each sample and manually selected the spectrum that was most representative of the sample. The term "most 
representative" indicates the spectrum segment in which all target peaks were seen at their maximum intensities relative to each other while retaining at least $10 \%$ of the original intensity of the calibrant peak. An integration algorithm in the instruments software was used to obtain drift times, reduced mobilities, and peak heights.

\subsubsection{Instrumentation}

The ion mobility spectrometers used were the Smith's Ionscan-LS® and Smith's Sabre $4000 ®$ (Smith’s Detection, Danbury CT USA). The Ionscan-LS® is a benchtop ion mobility spectrometer, while the Sabre $4000 \AA$ is a handheld field portable ion mobility spectrometer. Both instruments contain an internal nicotinamide calibrant. Routine maintenance procedures recommended by the manufacturer were conducted regularly during the length of the project. This included installing new air filter packs and membrane filters as well as cleaning the thermal desorption heaters and other accessible areas. Instruments remained powered on for the duration of the project unless errors results in the instruments being powered down or maintenance was being performed. The instruments were baked out each evening at elevated temperatures and flow to remove contaminants. Drift times were established and operating conditions optimized in positive mode for the selected standards at stock concentrations (Table 2.2).

Table 2.2 Operating parameters and conditions of the ion mobility spectrometers used for this study.

\begin{tabular}{rcc}
\hline & Ionscan ${ }^{\circledR}$ & Sabre $4000 \AA$ \\
\hline Drift Tube Temperature & $250{ }^{\circ} \mathrm{C}$ & $140{ }^{\circ} \mathrm{C}$ \\
Inlet Temperature & $290{ }^{\circ} \mathrm{C}$ & $140{ }^{\circ} \mathrm{C}$ \\
Desorption Temperature & $260{ }^{\circ} \mathrm{C}$ & $180{ }^{\circ} \mathrm{C}$ \\
Analysis Delay & $0.025 \mathrm{~s}$ & $0.100 \mathrm{~s}$ \\
Scan Period & $20 \mathrm{~ms}$ & $25 \mathrm{~ms}$ \\
Shutter Grid Width & $0.200 \mathrm{~ms}$ & $0.300 \mathrm{~ms}$ \\
Analysis Duration & $20 \mathrm{~s}$ & $30 \mathrm{~s}$ \\
\hline
\end{tabular}




\subsubsection{Quality Control}

One of the challenges of utilizing IMS as a screening device is assuring that the instrument is providing consistent and reliable data (drift time and peak intensity). Instrument validation samples are clearly important in this role, but such samples are designed to indicate that the instrument is functional as opposed to meeting a more stringent application-based requirement. As a detection method for OGSR in which multiple mobility peaks are anticipated and the background matrix (skin) is complex, additional QA/QC samples and practices are needed. Here, a control chart was constructed using 2,6-di-tert-butylpyridine (DtBP) to monitor instrument performance. DtBP has been suggested in literature as a chemical standard in IMS due to its mobility being independent of drift gas temperature, moisture, and electric field strength. ${ }^{16,17}$

Performance was characterized by peak height obtained when analyzing $5.0 \mathrm{ng}$ of DtBP. The Sabre's ${ }^{\circledR}$ (handheld) control chart's warning and control limits were established using results from 18 runs over an 8 day period ( 9 on one day followed by runs over the remaining days). This allowed for capturing inter- and intra-day variations in peak intensity. Once the control chart was constructed, the same amount of DtBP was analyzed daily and the resulting peak height charted on the control chart. If the signal fell outside of the upper and lower control limits (UCL and LCL), a second DtBP sample was analyzed and, if persistent, sample analysis was not conducted until maintenance was performed on the instrument and signal fell within the limits. The chart for the handheld instrument is shown in Figure 2.2. The thresholds established were more demanding that the instrument verification challenge and provided some measure of repeatability and reproducibility over the time in which the samples were analyzed. The chart 
also showed when routine maintenance was needed. The control chart for the benchtop instrument, similarly constructed, is provided in Appendix A.

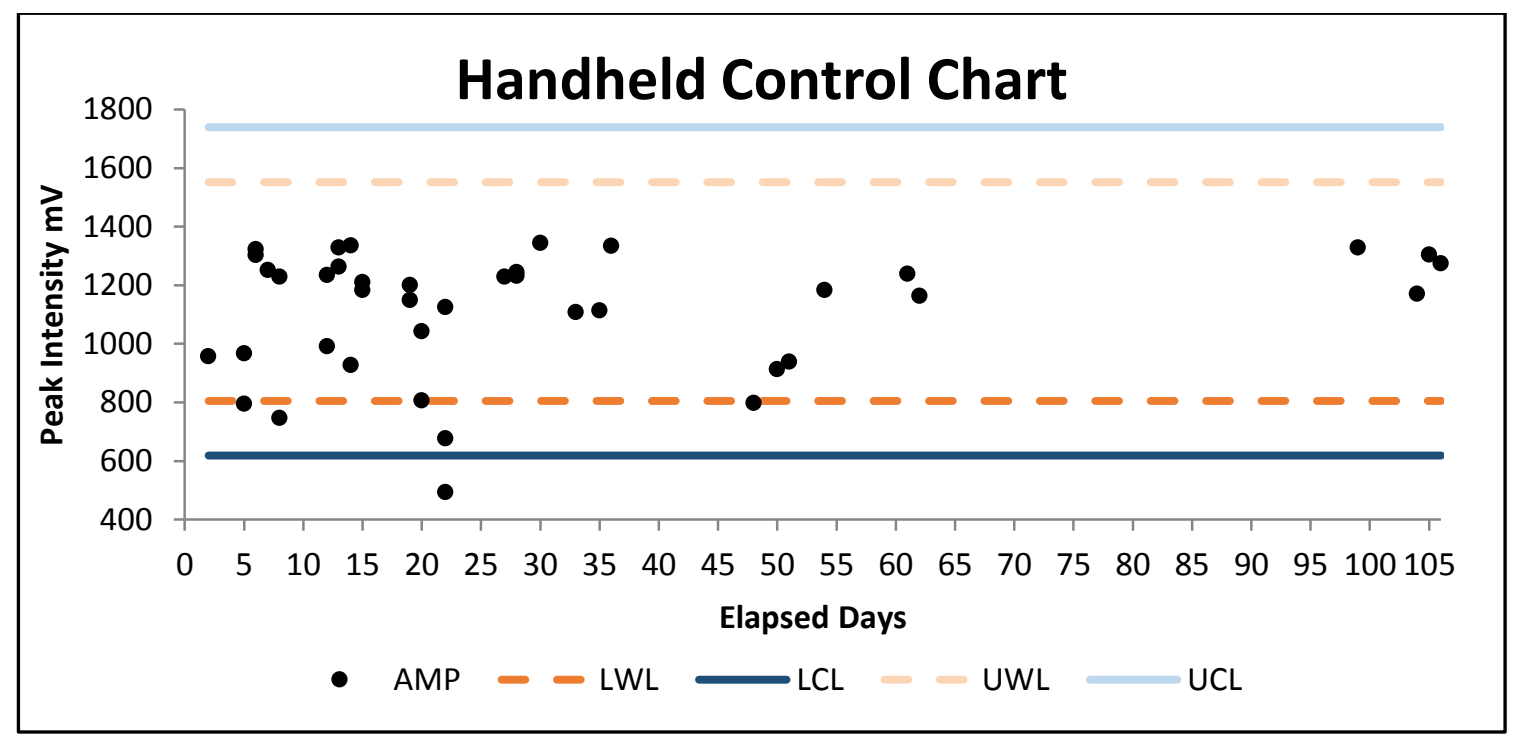

Figure 2.2 Control charts for the Sabre ${ }^{\circledR}$ handheld the horizontal lines indicate the calculated warning and control limits (AMP - amplitude, LWL - lower warning limit, LCL, lower control limit, UWL - upper warning limit, UCL - upper control limit).

\subsection{Validation Results \& Discussion}

\subsubsection{Detection Thresholds}

Ion mobility spectrometry using field instrumentation is semi-quantitative and for a presumptive assay such as for OGSR, determining specific limits of detection and quantitation (LOQ/LOD) is not directly applicable. Rather, it is preferable to generate a threshold of detection above which a signal would be considered sufficiently above the baseline to be associated with a positive response. This is small but important differentiation. The guidelines of 3- and 10- times signal to noise still have utility but they do not correspond to the strict definition of LOD/LOQ values as in other quantitative assays. 
Part of the challenge of establishing detection thresholds is defining what a detectable signal is and how reproducible that signal is over time. In IMS, the background signal is not necessarily uniform and can be dependent on the spectral regions as well as normal variations over time. One option is to determine the noise at every recorded drift time using intensities obtained from blank and background readings. This idea was dismissed for two reasons; first, it is impracticable given that drift times shift as a function of atmospheric conditions and thus vary over time, and second, it is unduly rigorous in the context of a screening application using a semi-quantitative method. An alternative was developed that has the added advantage of providing a means of estimating a detection threshold for each block of spectra studied.

As seen in Figure 2.3, example spectra show minimal signal in the drift time window from $1.0-7.0 \mathrm{~ms}$. Even when samples are introduced, it is unlikely that peaks will appear in this window as such species would have a smaller collisional cross-section that a reactant ion $(\mathrm{H} 2 \mathrm{O})_{\mathrm{n}} \mathrm{H}^{+}$. Therefore, this spectral region can be used to estimate the instrumental background signal (noise) and to define thresholds for peak detection within the spectrum of interest. This threshold was determined for several types of mobility spectra as summarized in Table 2.3. For these calculations, any negative intensity values were replaced with zero to avoid underestimating the mean signal and the standard deviation of the sample. 


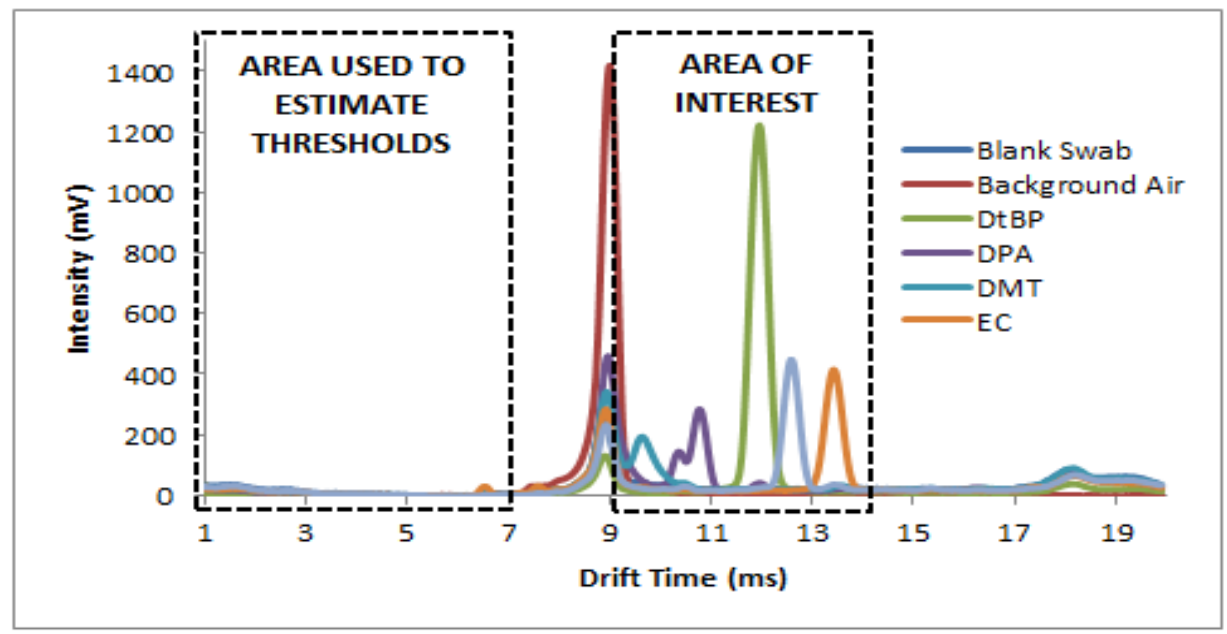

Figure 2.3 Full range mobility spectra (1-20ms) obtained with the Ionscan®. The $\mathrm{x}$-axis is drift time in milliseconds (ms) and the y-axis is the peak intensity in millivolts $(\mathrm{mV})$.

Table 2.3 Established IMS instrument critical thresholds a. is the Sabre's ${ }^{\circledR}($ handheld) \& $\mathbf{b}$. is the Ionscan's ${ }^{\circledR}$ (benchtop) thresholds.

\begin{tabular}{|c|c|c|c|c|c|c|}
\hline \multirow[t]{5}{*}{ a. } & & $\begin{array}{c}\text { Instrument } \\
\text { Blanks }\end{array}$ & $\begin{array}{l}\text { Media } \\
\text { Blanks }\end{array}$ & $\begin{array}{c}\text { Control } \\
\text { Chart }\end{array}$ & Skin Swabs & $\begin{array}{c}\text { Shooter } \\
\text { Swabs }\end{array}$ \\
\hline & \# points (n) & 7,653 & 5,061 & 8,435 & 40,970 & 4,097 \\
\hline & $\mathbf{X}(\mathbf{m V})$ & 0.45 & 0.8 & 1.0 & 1.4 & 3.1 \\
\hline & $\mathbf{S}(\mathrm{mV})$ & 0.82 & 1.2 & 1.5 & 2.0 & 4.1 \\
\hline & $X+3 * s$ & 2.9 & 4.3 & 5.4 & 7.3 & 15.3 \\
\hline \multirow[t]{5}{*}{ b. } & & $\begin{array}{c}\text { Instrument } \\
\text { Blanks }\end{array}$ & $\begin{array}{l}\text { Media } \\
\text { Blanks }\end{array}$ & $\begin{array}{c}\text { Control } \\
\text { Chart }\end{array}$ & Skin Swabs & $\begin{array}{c}\text { Shooter } \\
\text { Swabs }\end{array}$ \\
\hline & \# points (n) & 8,676 & 10,845 & 10,604 & 10,604 & 8,917 \\
\hline & $\mathbf{X}(\mathbf{m V})$ & 0.6 & 4.3 & 2.5 & 3.1 & 3.1 \\
\hline & $\mathbf{S}(\mathbf{m V})$ & 2.2 & 6.4 & 3.7 & 4.6 & 5.4 \\
\hline & $X+3^{*} \mathrm{~s}$ & 7.3 & 23.3 & 13.4 & 17.0 & 19.3 \\
\hline
\end{tabular}

For interpretative purposes, it was assumed that signals above the mean +10 s threshold would be unambiguously detectable with field instruments and those below the mean $+3 \mathrm{~s}$ threshold would be undetectable with signals between becoming increasingly difficult to detect as the $+3 \mathrm{~s}$ line was approached. For example, assume a skin swab is obtained to be used as a matrix control sample. It would be reasonable to cite a positive response for a peak if that response exceeded $7.3 \mathrm{mV}$ if analysed with the Sabre® ${ }^{\circledR}$ or $17 \mathrm{mV}$ if analysed on the Ionscan ${ }^{\circledR}$ as 
per the highlighted cells in Table 2.3 a and $\mathbf{b}$. Similar thresholds can be determined for any IMS instrument but they would have to be generated for each independently. These numbers are not transferrable nor would they be expected to be the same over a long period of time (months). Based on these considerations, the following procedure was used to estimate the detection threshold of the 4 target compounds. A data file was prepared that contained the daily background along with all spectra associated with a calibration study. The detection threshold for that compound was established as the concentration at which the intensity $(\mathrm{mV})$ exceeded that of the mean background (1-7 ms across all spectra in the constructed data file) plus three times the standard deviation (sampling) of the background intensity. This was accomplished using MatLab® and an example is shown in Figures 2.4. Additional figures are provided in Appendix A and the detection thresholds derived are summarized in Table 2.4.

Table 2.4 Amount deposited compared to estimated detection thresholds for the compounds of interest based on the established critical thresholds.

\begin{tabular}{rccc}
\hline & Deposited $^{*}$ & Ionscan $^{\circledR}$ & Sabre ${ }^{\circledR}$ \\
\hline DPA & $115 \mathrm{ng}$ & $1 \mu \mathrm{g}$ & $50 \mathrm{ng}$ \\
DMT & $90 \mathrm{ng}$ & $0.5 \mu \mathrm{g}$ & $5 \mathrm{ng}$ \\
EC & $178 \mathrm{ng}$ & $10 \mathrm{ng}$ & $1 \mathrm{ng}$ \\
MC & $\mathrm{X}$ & $10 \mathrm{ng}$ & $10 \mathrm{ng}$ \\
\hline
\end{tabular}

*Amounts deposited retrieved from Moran, J.W. and S. Bell, Skin Permeation of Organic Gunshot Residue: Implications for Sampling and Analysis. Analytical Chemistry, 2014. 86(12): p. 6071-6079. 


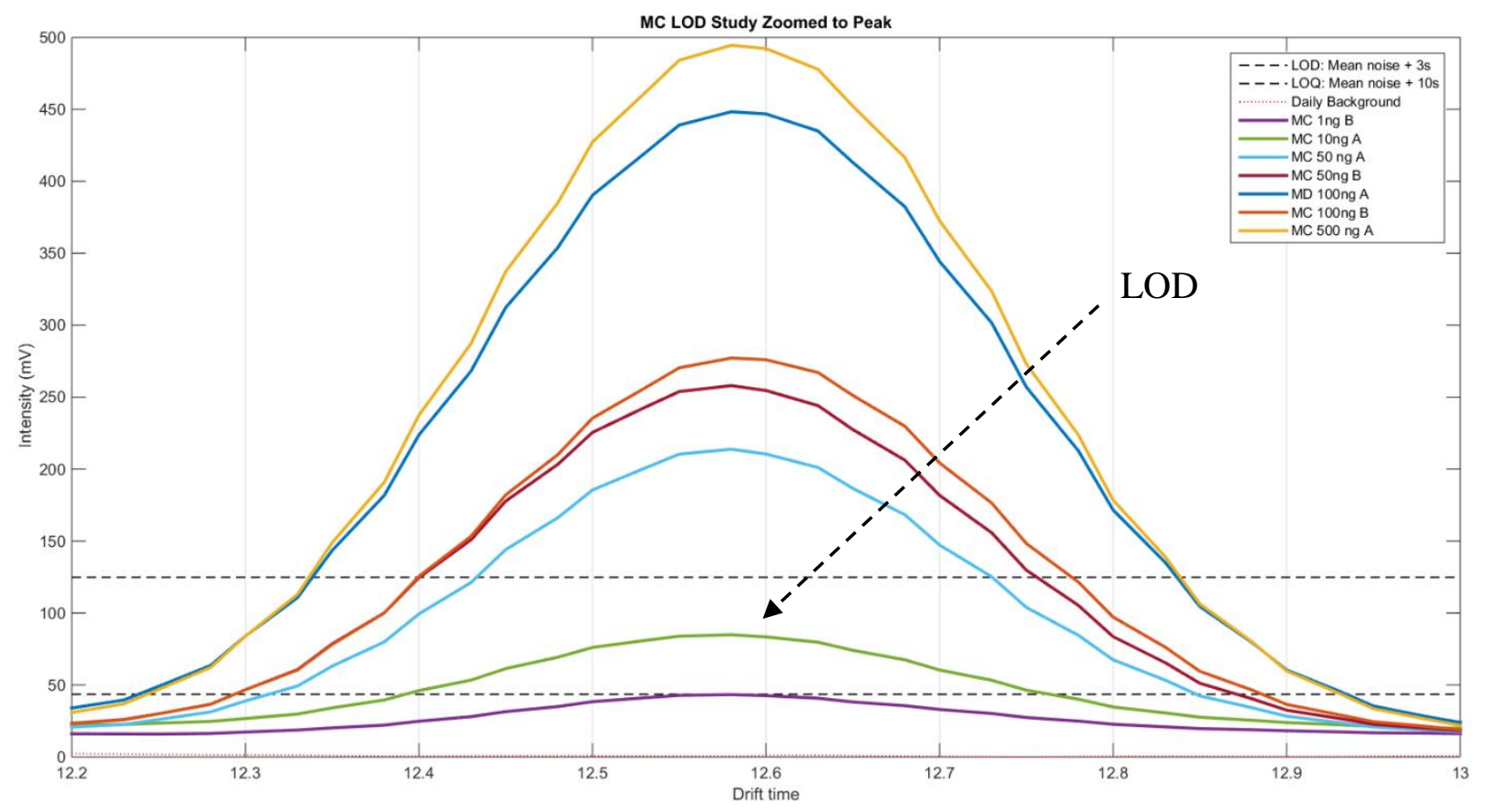

Figure 2.4 Zoomed to MC mobility peak in which the detection threshold is indicated.

Work previously published from our lab provided estimated deposition amounts for DMP, DPA, and EC as seen in Table 2.4. ${ }^{9}$ Detection limits for the handheld are below the amounts deposited by an authentic firing event for these three compounds whereas the thresholds for the benchtop instrument for DMP and DPA were higher than what would be expected to be present on a swab as a result of an authentic firing event. Thus, establishing detection thresholds will also be instrument-dependent. The reason that these thresholds were high in this case are not directly apparent but potential explanations can be offered. First, the size of the surface area sampled by the benchtop was slightly smaller than that of the handheld given that swabs had to be cut and placed on the desorber surface of the benchtop. Second, the temperatures used for the benchtop (Table 2.2) were purposely set differently to capture contributions to ruggedness and robustness of the technique. Third and probably most significant were differences in instrument history. As noted previously, both instruments were several years old at the time of the validation and both had different usage and history and thus performance state. The control chart for the 
benchtop (Appendix A) shows a greater variability and degradation of performance over time compared to the handheld instrument Figure 2.2. This type of wear and tear will be a factor with any field deployed instrument, which itself is strong argument supporting the use of a control chart as part of QA/QC. It is worth noting that when samples collected from known shooters were collected and analyzed (discussed in a later section), a mobility peak consistent with DPA was frequently seen, indicating that these detection thresholds are acceptable and fit-for-purpose.

\subsubsection{Repeatability \& Reproducibility}

Repeatability samples were prepared for each compound and analyzed immediately after preparation. Five were analyzed on the Sabre ${ }^{\circledR}$ and 15 on the Ionscan ${ }^{\circledR}$. Thirty reproducibility samples were prepared and stored at room temperature until analyzed. Three of the samples were analyzed twice a day for a total of 5 days (inter-day variation). To analyze sample stability

three samples from each storage location were analyzed simultaneously twice a week for $\sim 2$ weeks (intra-day variation). A total of 24 samples were prepared to be analyzed; 12 were stored in a freezer at a temperature of approximately $-26^{\circ} \mathrm{C}$ and 12 were stored at room temperature.

The repeatability and reproducibility of the method were measured as the intra and interday variability, respectively, in the drift time, peak intensity (amplitude), and reduced mobility $\left(\mathrm{K}_{\mathrm{o}}\right)$. Values were obtained using the "Gaussian fit" function on the software supplied with the instruments. Given that the instrument has an internal calibrant for adjusting drift times, the $\%$ RSD of drift times for all compounds across both instruments was consistently $<1 \%$. Of greater interest in this study was the repeatability and reproducibility of the peak amplitudes over time (Table 2.5) as this will have a direct impact on detection thresholds. 
Table 2.5 Repeatability (intra-) and reproducibility (inter-) of peak intensities (mv, \%RSD)

\begin{tabular}{lcccc}
\hline & \multicolumn{2}{c}{ Ionscan ${ }^{\circledR}$} & \multicolumn{2}{c}{ Sabre ${ }^{\circledR}$} \\
\hline Inter- & Intra- & Inter- & Intra- \\
\hline DPA & $22 \%$ & $40 \%$ & $52 \%$ & $27 \%$ \\
EC & $29 \%$ & $31 \%$ & $5.1 \%$ & $23 \%$ \\
MC & $22 \%$ & $11 \%$ & $11 \%$ & $34 \%$ \\
\hline
\end{tabular}

The data shows no particular trends other than the large variation of peak intensity over time. The largest variations (52\% and $40 \%)$ are observed for DPA, which is the most volatile of the compounds but the analogy does not carry to DMT which is also relatively volatile but has the lowest inter-day variation reported. Many factors are involved including instrument variation, variation in sample preparation, and atmospheric conditions. As applied here, this variation is not a limiting factor as long as it is taken into consideration. For determining the detection thresholds as described in the previous section, the method used for establishing the instrument background was designed to capture some of this variation over time. The other available tool is the control charting also as described above.

\subsubsection{Sample Stability}

Stability of samples collected on wipes was evaluated as a function of storage conditions - room temperature and laboratory lighting vs. $-26{ }^{\circ} \mathrm{C}$ and dark. The data from the Ionscan® is shown in Figure 2.5; data from the Sabre ${ }^{\circledR}$ was comparable. Signal degradation is evident under both storage conditions but most pronounced at room temperature conditions (dotted lines). While variation is evident, this is not surprising based on the reproducibility findings. 
Regardless, the trend is clear and the degree of signal degradation correlates with vapor pressure (Table 2.1). In the case of DMT, the most volatile compound, signal degrades rapidly even under cold and dark storage conditions. Conversely, EC, least volatile, degrades but remained detectable up to 9 days. DPA, which is anticipated to be one of the critical compounds for any OGSR assay, degraded below detectability between 7 and 9 days at room temperature but remained relatively stable under cold dark storage conditions.

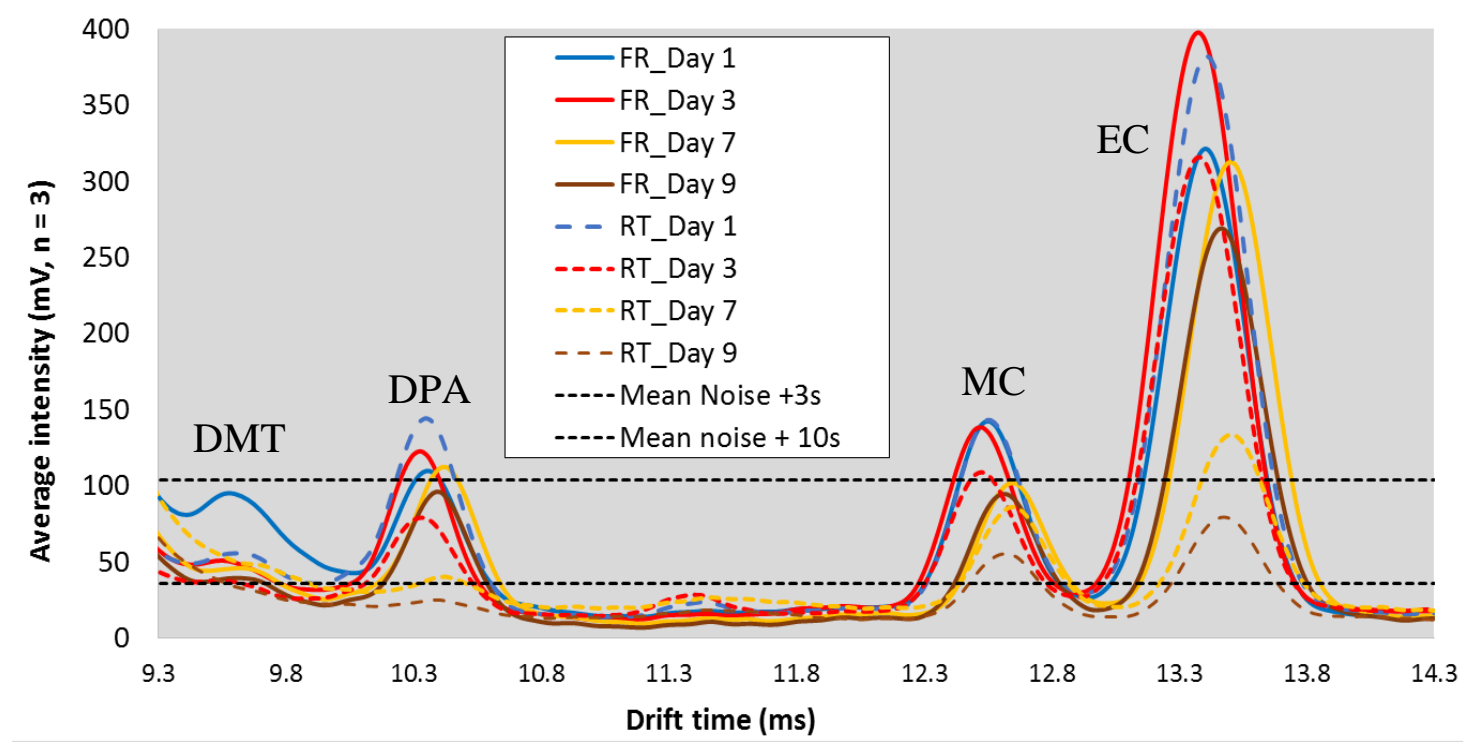

Figure 2.5 Average mobility spectra obtained with the Ionscan ${ }^{\circledR}$ in which stability is a function of storage conditions. Solid lines indicate samples stored in the freezer (FR) at $-26{ }^{\circ} \mathrm{C}$ whereas dashed lines are samples stored at room temperature (RT).

To incorporate a measure of variability, the maximum peak height for each mobility spectrum was retrieved from the stored XY data file. The three maxima for each day were averaged and the 95\% confidence interval was calculated. Results are shown in Figures 2.6-2.8. The data obtained from samples stored in the freezer was broken down into two figures (Figures 2.6 and 2.7) for clarity. Data obtained from samples stored at room temperature is presented in a combined figure (Figure 2.8). The range for samples stored in the freezer (Figures 2.6 and 2.7) are generally larger than those stored at room temperature (Figure 2.8). Given that the control 
charting and other QA/QC procedures indicated that the instrument was performing acceptably and consistently, the reason for the variability seen in the samples stored in the freezer is not immediately obvious. In part, this may be due to analyte migration on the muslin which would be a factor given that only a small portion of the muslin wipe was subject to thermal desorption. Statistical significance tests were not applied to the data but qualitative review of the data in

Figures 2.6 and 2.7 (stored in the freezer) suggests that degradation is occurring during the last few days of the study.

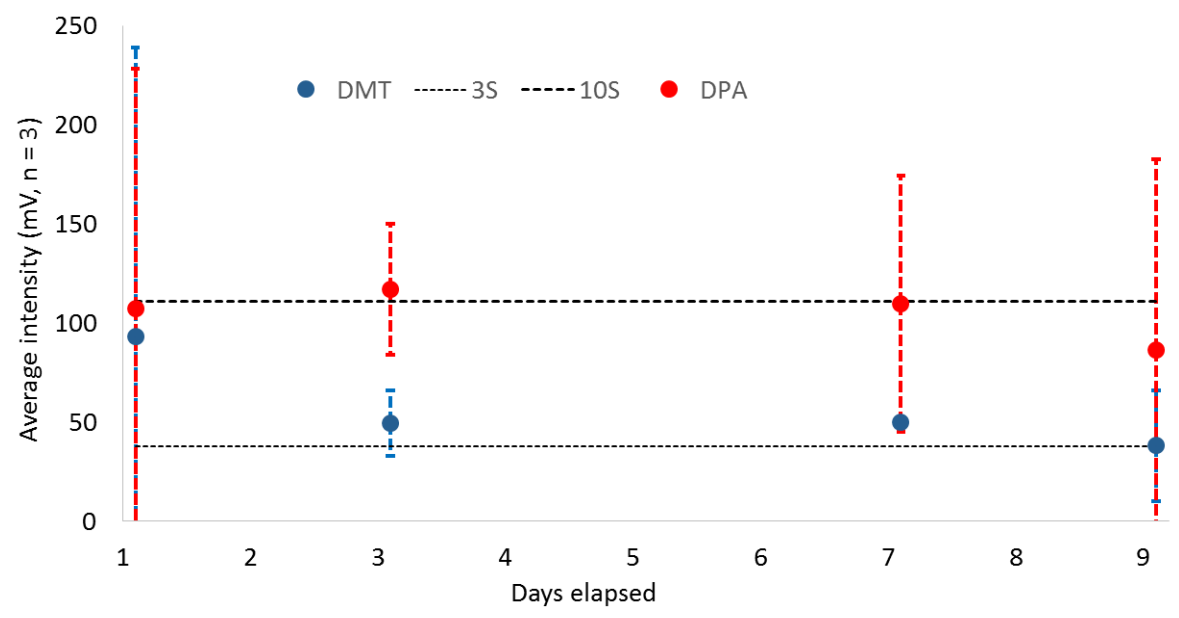

Figure 2.6 Average maximum peak intensity $(n=3)$ for DMT and DPA; samples stored in freezer. The $x$ axis is the number of days elapsed since preparation and the y-axis is the intensity. The error bars correspond to the $95 \%$ confidence interval for $n=3$.

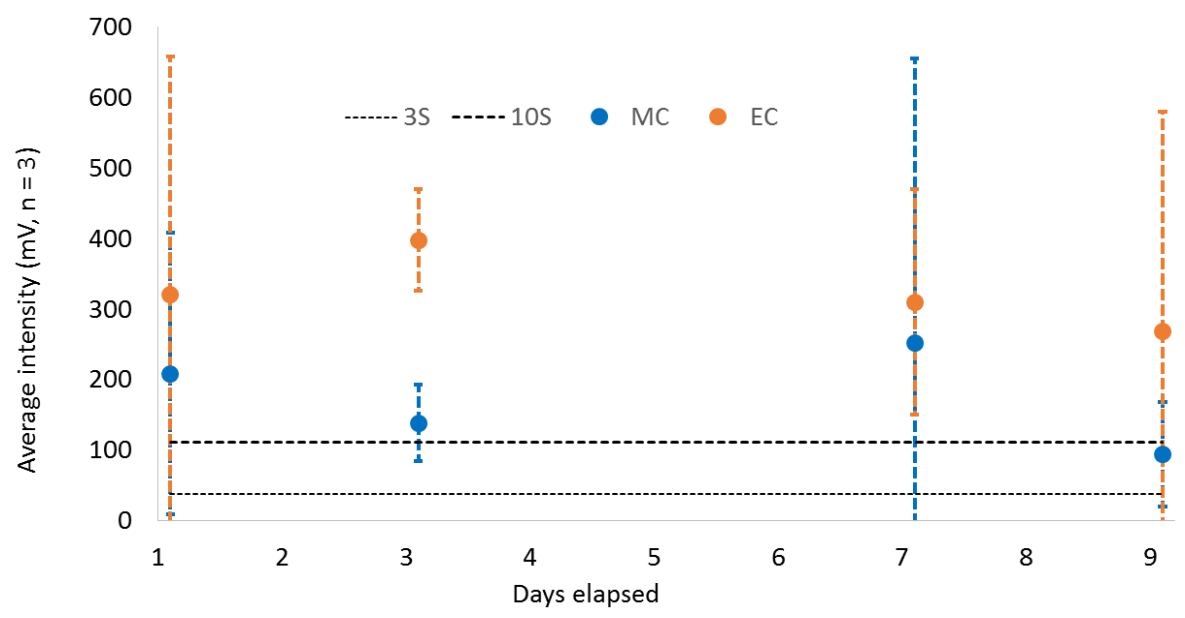

Figure 2.7 Average maximum peak intensity (n=3) for MC and EC; samples stored in freezer. Axes and error bars are as stated in Figure 2.6. 


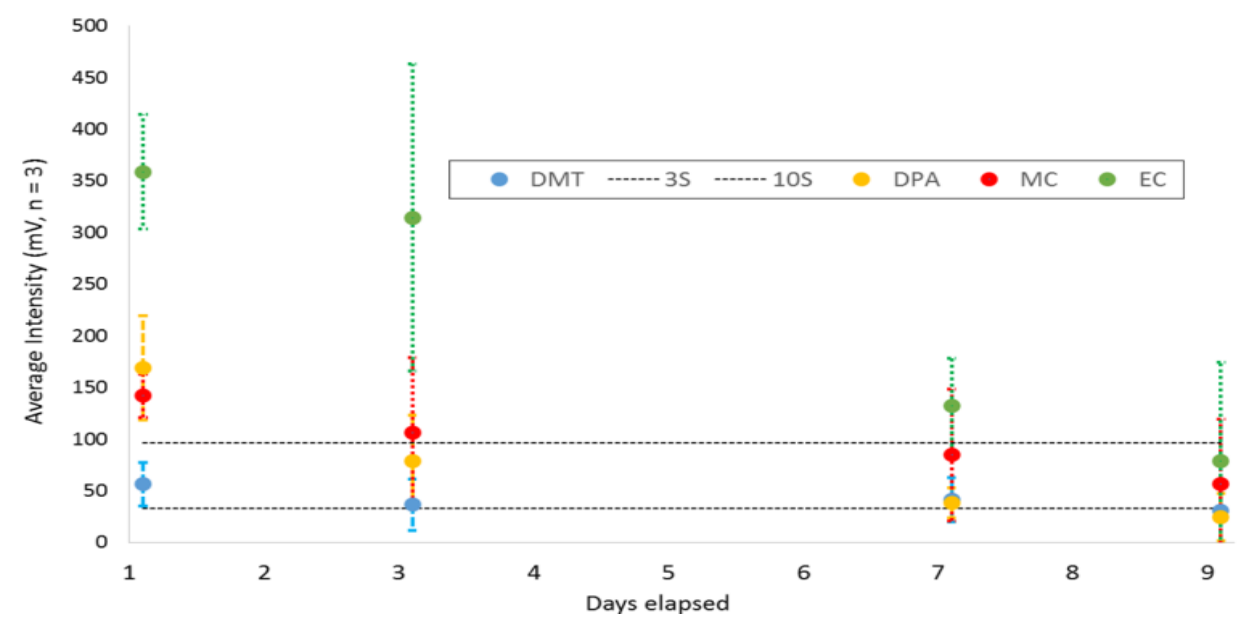

Figure 2.8 Average maximum peak intensity $(n=3)$ for all four target compounds; samples stored at room temperature. Axes and error bars are as stated in Figure 2.6.

There is little subtlety associated with the data obtained from samples stored at room temperature (Figure 2.8). The trend for all four compounds and the signal for both DPA and DMT drops below the $3 \mathrm{~s}$ line by day 9 . To further characterize the results, a natural logarithm fit was applied to the data (not shown). This is similar to the approach used in estimating timesince-discharge based on samples collected from spent cartridges. ${ }^{7,18,19}$ Because the DMT signal was close to the $3 \mathrm{~s}$ limit and the fit was relatively poor $\left(\mathrm{R}^{2}=0.73\right)$, no additional analysis was applied to this compound. The signal degradation of the remaining compounds reasonably approximates a first-order process and as such allows for estimation of rate constants and halflife:

$$
t_{1 / 2}=\frac{0.693}{k}
$$

where $\mathrm{k}$ is the rate constant as obtained from the slope of the fitted curve. Based on this, the half-life of each compound can be estimated based on room temperature storage conditions. For DPA, the half-life is approximately 3 days, 3.6 days for EC, and 6.7 days for MC. 
The estimated half-life is not meant to be used as holding time limits per se, but as a guide for establishing these limits under room temperature storage conditions. Because DPA is one of the most frequently targeted compounds in OGSR assays, storage under cold dark conditions with holding times of less than a week are recommended when muslin wipes or similar substrates are used.

\subsection{Application to Authentic Samples}

Samples collected from known shooters and from a general population study $(\mathrm{n}=171$, IRB approved $\ddagger$, were analyzed using optimized conditions on one of the two instruments. The challenge of this application is not the ability to detect the compounds of interest; rather it is being able to detect them in the presence of the background of a skin swab. Because the IMS instruments used in this study use thermal desorption for sample introduction, skin swabs generate a complex mixture of compounds being introduced into the ionization region to accompany OGSR compounds present. Competitive ionization and gas phase basicity are key considerations in determining what ion/molecule clusters form and are detected (positive mode). Add to this the inherent variability expected between individuals and what is recoverable from their hands at any given time, and the difficulty of the analytical problem is evident. Despite this, initial results were promising.

In about $70 \%$ of the shooter samples, it was possible to detect peaks in the drift time windows associated with DPA, n-nitrosodiphenylamine (evaluated in earlier work in our laboratory), and DMT using either instrument. However, it was not possible to program a series of mobility peaks that could be interpreted collectively as either consistent with a shooter or consistent with a non-shooter. The results are summarized in Figures 2.9 and 2.10. The upper frame of Figure 2.9 shows the drift times of interest for the portable instrument with individual 
plots representing the mean of subject samples (the population background samples, $n=170$ ), known shooters $(n=7)$, laboratory backgrounds $(n=33)$, and the daily control chart samples $(n=35)$ for reference. The drift time range of 12-13.5 ms shows the most obvious differences between samples from shooters and the general population. This is the drift time that incorporates DPA although this identification cannot be considered definitive. The lower frame of the same figure plots the \%RSD of the $\mathrm{mV}$ intensity values of the averaged spectra. At nearly every drift time, the variation in spectra as measured by the \%RD is greater than the variation seen in the control shooter samples. Figure 2.10 depicts the same information for the benchtop instrument with an added dataset obtained from muslin blanks. The mobility window near 9.5 ms also corresponds to DPA. The number of averaged spectra were subject $(n=143)$, known shooters $(n=38)$, laboratory backgrounds $(n=37)$, muslin blanks $(n=46)$, and daily control chart samples $(\mathrm{n}=45)$.
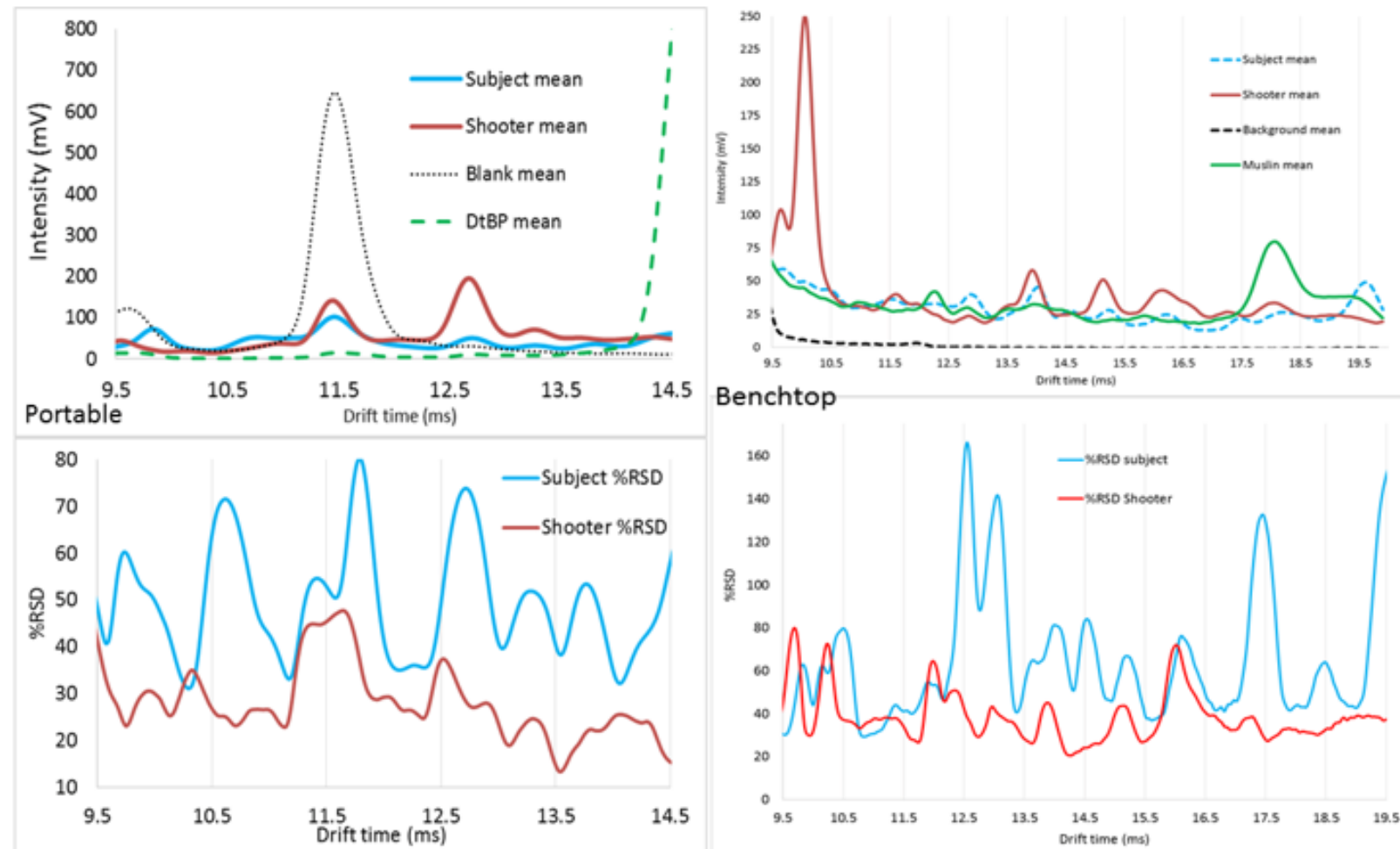

Figure 2.9 Summarized results for authentic samples

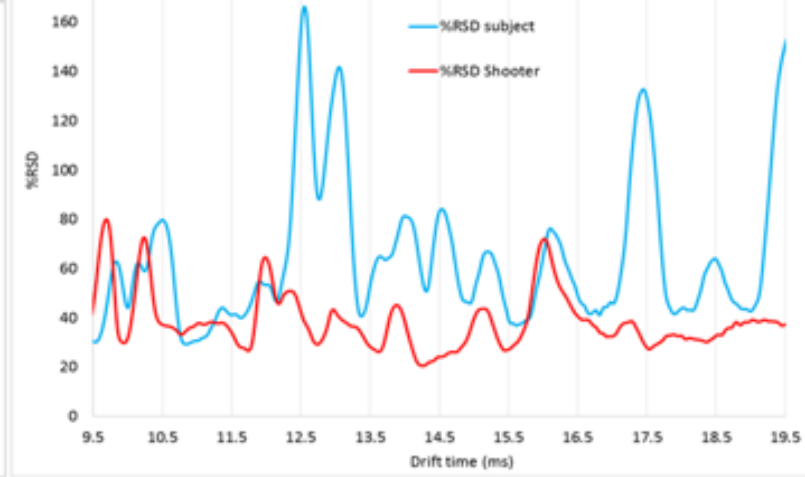
analysed with the Sabre $\mathbb{B}$ (handheld/portable) instrument.

Figure 2.10 Summarized results for authentic samples analysed with the Ionscan $($ benchtop) instrument. 
The significant variability across the population sampled is not surprising and characterizing is vital for moving any technique into practice. In Figure 2.9, the feature with a drift time of $\sim 12.5 \mathrm{~ms}$ is distinctive for the shooter profile and also corresponds to one of the smaller \%RSD values which suggests that this feature is fairly consistent across all shooters. What complicates the interpretation is the relatively high variability of subject hand swab spectra in the same drift time window. The pattern seen in Figure 2.10 shows less variability of the subject samples compared to the shooter samples and also shows evidence of other spectral features that appear to be more consistent in shooters vs. subjects $(\sim 13.5,14.2$, and $16.2 \mathrm{~ms}$ for example). It is worth noting that different sets of samples were analysed on each instrument so that results from both need to be considered together. Collectively, these results argue for a pattern-based analysis rather than relying on a combination of distinct peaks for characterizing shooters vs. non-shooters. As seen in Figure 2.11, the differences between spectra collected from the hands of shooters has discernible differences from all other types of spectra; some are obvious and some, such as seen at later drift times, are more subtle. Work is underway in our laboratory pursuing this alternative.

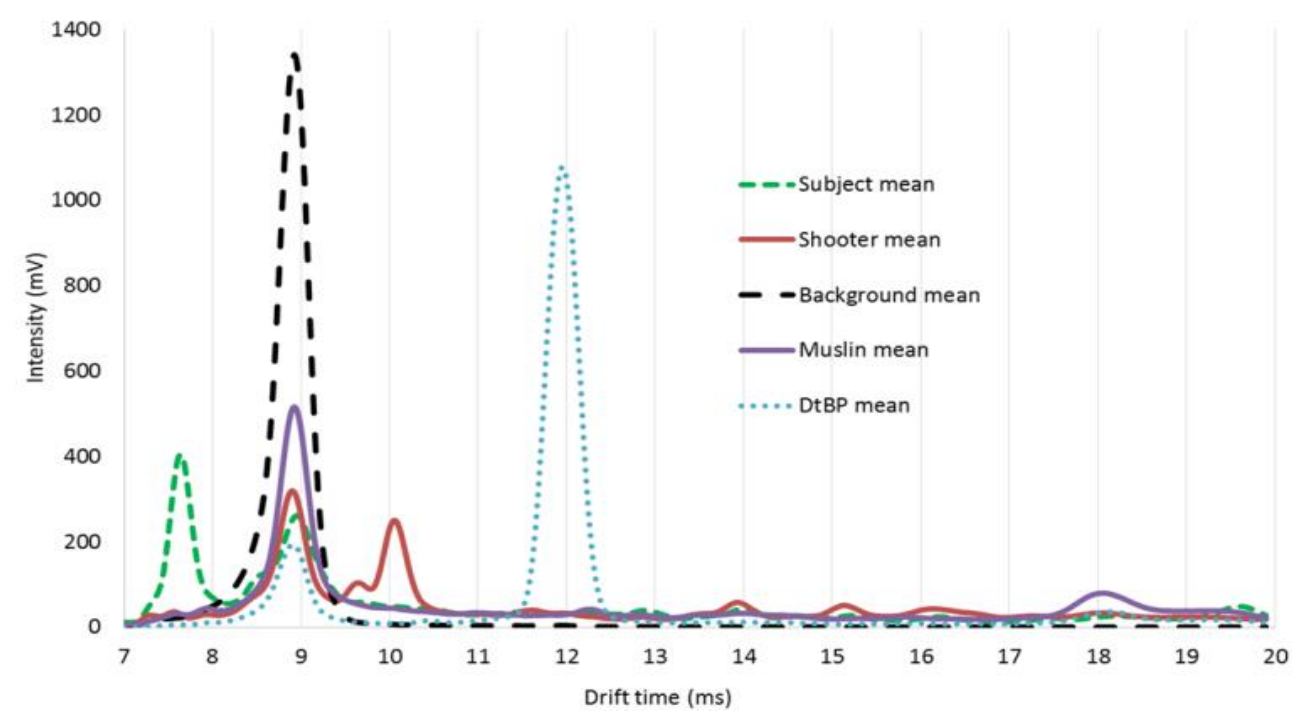

Figure 2.11 Spectra indicating the differences between hands of shooters and other collected spectra. 


\subsection{Conclusion}

IMS shows promise as a screening test for OGSR recovered from hand swabs although the matrix continues to present a challenge. Two instruments were evaluated here with comparable results. Figures of merit established here can be considered representative but could not be generalized to all instruments although the general procedure outlined here could. The key to successful deployment of IMS this role will be the use of QA/QC beyond performance verification standards and the adoption of pattern-based data analyses rather than identification of specific compounds and mobility peaks associated with them. To address variability in peak intensity over time, some type of daily monitoring will be essential and will have to be validated on each instrument. The control chart approach suggested here is well-suited to this task. For OGSR, a second control compound could be used such as DPA to insure that the inherent variations in signal intensity are captured and considered given that sample stability is clearly a contributor to inter-day variation. Clearly sample holding times will be a consideration and samples for OGSR will likely require different and more stringent storage conditions than sample collected for traditional GSR analysis. This study suggests holding time limits in days or a few weeks, but this cannot be generalized past these compounds on the sampling media used for this study.

\subsection{Funding and Additional Notes}

This work was funded through a "National Institute of Justice Forensic Technology Center of Excellence” project, award number \#2011-DN-BX-K564, RTI International (6-321-0213168).

Approval for human subject sampling was obtained through the West Virginia University Institutional Review Board (IRB), protocol 1209000337. 


\subsection{References}

1. B. J. Heard, Forensic Ballistics in Court : Interpretation and Presentation of Firearms Evidence, John Wiley \& Sons, Somerset, NJ, USA, 2013.

2. T. Jalanti, P. Henchoz, A. Gallusser and M. S. Bonfanti, Sci. Justice, 1999, 39, 48-52.

3. Z. Brozek-Mucha, Microsc. microanal., 2011, 17, 972-982.

4. K. H. Chang, P. T. Jayaprakash, C. H. Yew and A. F. L. Abdullah, Australian Journal of Forensic Sciences, 2013, 45, 3-23.

5. $\quad$ O. Dalby, D. Butler and J. W. Birkett, J. Forensic Sci., 2010, 55, 924-943.

6. J. Arndt, S. Bell, L. Crookshanks, M. Lovejoy, C. Oleska, T. Tulley and D. Wolfe, Forensic Sci.Int., 2012, 222, 137-145.

7. C. Weyermann, V. Belaud, F. Riva and F. S. Romolo, Forensic Sci.Int., 2009, 186, 2935.

8. J. L. Thomas, D. Lincoln and B. R. McCord, J. Forensic Sci., 2013, 58, 609-615.

9. J. W. Moran and S. Bell, Analytical Chemistry, 2014, 86, 6071-6079.

10. J. Moran and S. Bell, Int. J. Ion Mobil. Spec., 2013, 16, 247-258.

11. M. X. Zhao, S. C. Zhang, C. D. Yang, Y. C. Xu, Y. X. Wen, L. S. Sun and X. R. Zhang, J. Forensic Sci., 2008, 53, 807-811.

12. G. A. Eiceman and Z. Karpas, Ion Mobility Spectrometry, CRC Press, Boca Raton FL, Second edn., 2005.

13. G. A. Eiceman, TrAC Trends in Analytical Chemistry, 2002, 21, 259-275.

14. G. A. Eiceman and J. A. Stone, Analytical Chemistry, 2004, 76, 390A-397A.

15. S. Bell, Forensic Chemistry, Pearson, 2012.

16. G. Kaur-Atwal, G. O’Connor, A. Aksenov, V. Bocos-Bintintan, C. L. Paul Thomas and C. Creaser, Int. J. Ion Mobil. Spec., 2009, 12, 1-14.

17. G. A. Eiceman, E. G. Nazarov and J. A. Stone, Anal. Chim. Acta, 2003, 493, 185-194.

18. J. Andrasko and S. Stahling, J. Forensic Sci., 2003, 48, 307-311.

19. M. Gallidabino, C. Weyermann, F. S. Romolo and F. Taroni, Sci. Justice, 2013, 53, 4148. 


\section{Chapter 3: Initial evaluation of inlet thermal desorption GC-MS analysis for organic gunshot residue collected from the hands of known shooters. ${ }^{1}$}

${ }^{1}$ Reproduced from Elsevier's Forensic Chemistry journal: Initial evaluation of inlet thermal desorption $G C-M S$ analysis for organic gunshot residue collected from the hands of known shooters. B. Stevens, S. Bell, and K. Adams, Forensic Chemistry 2, 55-62, 2016. http://www.sciencedirect.com/science/article/pii/ S2468170916300480

Permission was obtained from the co-authors and was not required by the journal according to Elsevier's Author and User Rights and permissions guidelines. The policy is located at the following web address: https://www.elsevier.com/about/company-information/policies/copyright/permissions\#Permission $\% 20$ Guidelines

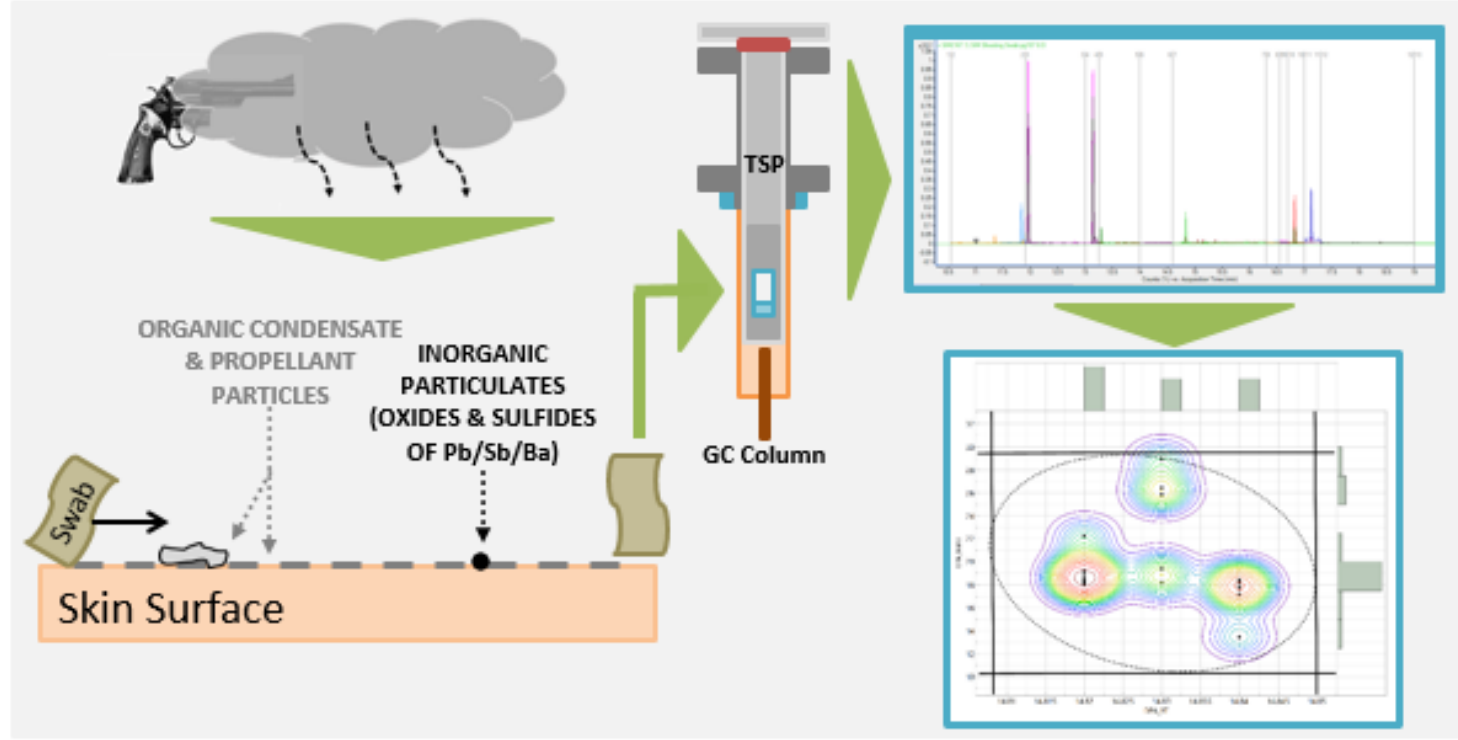

The discharge of a firearm produces a wealth of physical and chemical evidence. Traditional forensic analysis has focused on inorganic particulates formed from the primer, referred to as gunshot residue (GSR). The last few years have seen interest in expanding the list of target compounds to include organic constituents of firearm discharge residue (OGSR). To facilitate adoption by the forensic community, new assays ideally should exploit instrumentation commonly found in forensic laboratories such as gas chromatography/mass spectrometry (GC/MS). Here, a commercially available thermal separation probe that fits directly into the injection port was evaluated as a means of sample introduction for GC/MS operated in a selected 
ion monitoring mode. A statistical approach utilizing bivariate plots linked retention time to ion ratio data to afford a probabilistic interpretation of the results. A total of 27 authentic shooter swabs were collected after firing of 1-5 rounds and were analyzed in halves or triplicates. Ethyl centralite was detected in $81 \%$ of the samples; diphenylamine in $56 \%$, and 2-nitrodiphenylamine in $14 \%$. Dimethyl and dibutyl phthalates were detected in a majority of the swabs but also in many of the hand swab blanks. The use of surrogate standards provided a measure of recovery and reproducibility for retention times and ion ratios.

\subsection{Introduction}

Once a firearm is discharged, vapors and particles escape from the weapon in a radial expulsion known as a plume. The plume is a complex heterogeneous mixture referred to as firearm discharge residue (FDR) and is comprised of inorganic particulates (GSR), organic condensates, and particles of unburnt and partially burnt propellant (OGSR collectively). This mixture is deposited on proximate surfaces (Figure 3.1) including the hands, chest, and face of the individual firing the weapon.

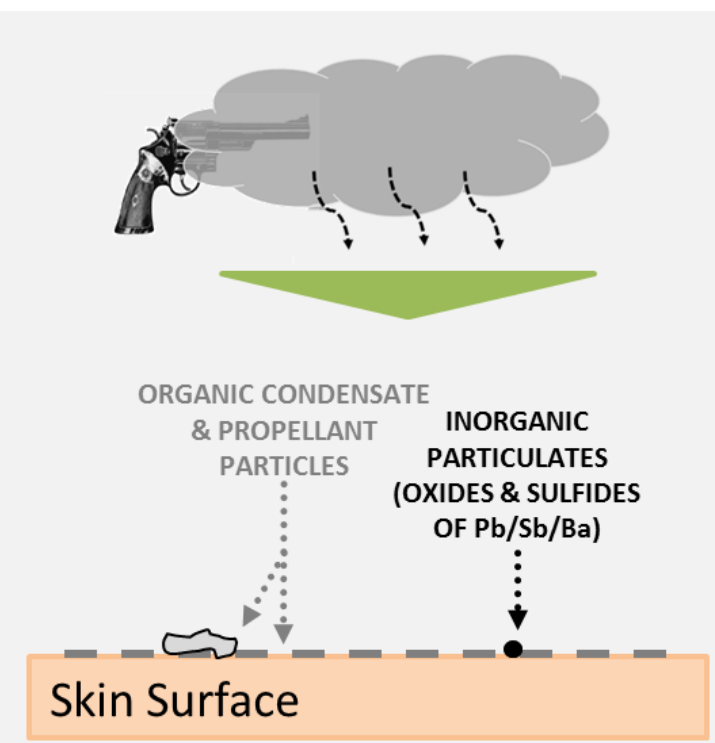


Figure 3.1 Deposition of firearm discharge residue including GSR and OGSR on the surface of the skin

Many variables dictate the amount of residue deposited including ammunition and weapon used and environmental conditions. In a recent study it was estimated that deposition amounts for OGSR compounds on the hands of shooters ranged from 90-178 ng total. ${ }^{1}$ Thus, it becomes essential to collect as much residue as possible over a large surface area (the hands) while concentrating what is collected in a relatively small area. Doing so facilitates the use of different strategies for sample preparation and extraction. Various methods have been used for sampling FDR including adhesive tapes, stubs, glues, swabbing, and vacuum lifts. ${ }^{2-5}$ Adhesive tapes and stubs are the most common and are typically used for collecting GSR particulates. Recent research has investigated the capability to utilize the same stub for collection of both GSR and OGSR compounds. ${ }^{5}$ Swabbing is the second most common technique for FDR sampling and is used for the collection of both inorganic and organic residues. Frequently, the swab is dipped in a suitable solvent prior to sampling to facilitate transfer of residues to the surface of the swab.

Current forensic methods of FDR detection focus on the inorganic particulates (GSR) originating from compounds found in the primer. Analysis using scanning electron microscopy (SEM) coupled with energy dispersive $\mathrm{x}$-ray spectroscopy (EDX) is a technically sound and vetted method detailed in an ASTM Method. ${ }^{6}$ SEM-EDX identifies GSR based on spherical morphology and inorganic composition. There is no issue with this methodology per se; however, the combination of organic and inorganic data would increase the value of any positive findings. The primary limitations of SEM/EDS include long analysis times as well as the cost and complexity of instrumentation. Particulate GSR evidence is prone to secondary transfer, meaning it can be transferred by physical activity or hand washing. Loss due to secondary 
transfer makes data interpretation more difficult as time passes. In addition, ammunition manufacturers have begun producing lead free ammunition (LFA) by replacing of lead with other metals such as copper, aluminum, and zinc, which results in changes in the composition of traditional GSR particulates. ${ }^{7}$ Although the ASTM method now includes additional elements found in LFA the absence of a traditional GSR inorganic component $(\mathrm{Pb}, \mathrm{Ba}, \mathrm{Sb})$ could lead to false negatives or inconclusive results. Finally, the GSR particulates make up a fraction of the discharge residue, leaving other potential chemical evidence unexamined. At the least, methods targeting organic constituents could serve as strong supporting evidence for the presence of FDR and could aid in addressing the limitations associated with GSR merely by itself.

Nitrocellulose (NC) and nitroglycerine (NG) are the main energetic components in small arms propellants. Although these two explosive components decompose upon discharge of a firearm, decomposition also occurs during storage. The decomposition, which particularly occurs in moist air or under hot conditions, forms nitric and nitrous acids which will further degrade the propellant. Ancillary compounds are added to propellants to function as stabilizers, plasticizers, flash suppressants, and deterrents. These include compounds such as ethyl centralite (EC), methyl centralite (MC), dimethyl phthalate (DMP), and diphenylamine (DPA). ${ }^{1,2,8-15}$ Nitration products of DPA including n-nitrosodiphenylamine (N-NDPA), and 2-, and 4nitrodiphenylamine (2-NDPA and 4-NDPA), formed as the energetic materials degrade have also been studied. ${ }^{1,2,8-15}$ Reviews by Dalby et.al. ${ }^{2}$ and Taudte et. al. ${ }^{15}$ and work done by Weyermann et. al. ${ }^{16}$ include more extensive lists of compounds that may contribute to OGSR. These lists include compounds such as carbazole, camphor, akardite, and cresol. ${ }^{2,15,16}$ These compounds have been the focus of much research as was the case with this work. 
Although methods utilizing liquid chromatography and mass spectrometry (LC/MS) and gas chromatography-mass spectrometry (GC/MS) have been applied to hand swabs, the methodology has not yet found widespread application in forensic laboratories. ${ }^{15}$ Solvent extraction of the swabs are generally multi-step processes that include drying and reconstitution in small volumes. Extractions are time consuming, destructive, and risk the introduction of contamination. Prepared with proper care, these extracts clearly can be used in conjunction with electrospray (ESI) and atmospheric pressure ionization (APCI) mass spectrometers coupled to mass spectrometers such as exact mass time of flight or triple quadrupole systems. ESI/MS systems are often available in toxicology sections but developing and validating methods on working instruments can be a challenge in a casework laboratory. Conversely, GC/MS instruments are ubiquitous in forensic laboratories, but limitations such as the small amount of FDR present, swabbing efficiency, and extraction efficiency tend to drive concentrations below detection limits for successful characterization of OGSR recovered by solvent extraction from swabs.

Solvent-less methods have also been examined for sample introduction for GC/MS in the context of OGSR. These methods rely on thermal desorption of compounds from the swab or other sample such as unburnt and partially burnt gunpowder particles, firearm barrels, and spent cartridge casings. ${ }^{11,14,16-20}$. In passive thermal desorption, the vapors are introduced directly into the GC inlet without additional pre-concentration. Alternatively, the vapors can be reconcentrated on a solid phase such as in SPME or variants such as stir bar, micro-drop, or planar surface sorption.

The use of passive thermal desorption (TD) to introduce OGSR samples into an instrument has been demonstrated previously in our laboratory. Ion mobility spectrometry (IMS) 
has been shown to be useful for differentiating known shooters from non-shooters when used as a screening test. ${ }^{8,9,21-23}$ With IMS, sampling media is directly inserted into the thermal desorber without any preparation or pre-treatment. Direct heat is applied to the swab, releasing volatile organic compounds (VOCs) from the sampling matrix into the ionization region of the instrument as a function of vapor pressure. The temperature is optimized to afford rapid desorption without inducting degradation of the sample media.

In this project, TD was directly coupled with gas chromatography mass spectrometry (GC/MS) using a commercially available specialized injection port fitting called a thermal separation probe (TSP). The probe assembly takes the place of the top nut of the injection port and the sample holder fits down into the inlet liner. Thus, the injection port acts as the thermal desorber. Volatilized compounds are swept into the column via carrier gas and concentrated on the head of the column which is maintained as close to room temperature as possible.

The TSP has been applied to FDR analysis before although not as a passive sample introduction system as described in this paper. Tarifa et. al ${ }^{24}$ and Fan et. al. ${ }^{25}$ described preconcentration methods in which vapors from samples, collected on cotton swabs, were trapped onto glass fiber filters coated with PDMS contained within a capillary. The capillary was then inserted into the TSP holder and desorbed onto the column. This method, called capillary microextraction of volatiles (CMV) was used along with LIBS spectroscopy to characterize swabs recovered from police officers and non-shooters. ${ }^{24}$ A combination of passive and active headspace methods were used to pre-concentrate the organics on the glass/PDMS filters which were in turn thermally desorbed using the TSP and characterized by GC/MS (or GC- $\mu \mathrm{ECD}$ ). The authors estimated detection limits for target compounds (NG, 2,4-DNT, and DPA) in the 
headspace to be in the range of $\sim 3$ to $9 \mathrm{ng}$ and when the method was applied to authentic shooting samples, NG and DPA were detected in 5 of the 9 known shooter samples.

The goal of this research was to evaluate the feasibility and performance characteristics of thermal desorption as a means of sample introduction for analysis of organic gunshot residue with GC-MS directly from hand swabs without prior extraction or pre-concentration. The advantage of this approach is that there is literally no sample preparation - swabs are loaded into a quartz micro-tube, placed in the TSP, and desorbed in the injection port. The challenge is detecting compounds from hand swabs at forensically-relevant amounts (1-3 shots). Here, selected ion monitoring was used to maximize sensitivity and selectivity of the instrumental system. Surrogate standards were spiked onto each swab at a known concentration just prior to analysis and used to gauge the efficiency of the desorption process.

\subsection{Experimental Section}

\subsubsection{Sample Preparation}

Working standard solutions of DPA, 2-NDPA, 4-NDPA, EC, 2,4-DNT, DBP, and dimethyl phthalate (DMP) were prepared at approximately $10 \mathrm{mg} / \mathrm{mL}$ from analytical grade solids in methanol (0.2 micron filtered) purchased from Fisher Scientific $®$. A working solution of carbazole at approximately $5 \mathrm{mg} / \mathrm{mL}$ was prepared in ethanol purchased from Sigma Aldrich ${ }^{\circledR}$. These standards were obtained from various chemical companies. Solid standard of methyl centralite was unavailable and thus a standard solution at $0.1 \mathrm{mg} / \mathrm{mL}$ in a mixture of acetonitrile \& methanol was obtained from Accustandard®. A standard solution of EPA Method 529 Surrogate at $1 \mathrm{mg} / \mathrm{mL}$ in methanol was also obtained from Accustandard $®$. Dilutions of the standards were prepared in the above mentioned methanol and ethanol in the case of carbazole. The chosen surrogate is a mixture of two compounds (1,3,5-trimethyl-2-nitrobenzene and 1,2,4- 
trimethyl-5-nitrobenzene) and is used in the U.S. Environmental Protection Agency's method 529, Determination of Explosives and Related Compounds in Drinking Water by Solid Phase Extraction and Capillary Column Gas Chromatography/Mass Spectrometry (GC/MS), EPA/600/R-05/052. ${ }^{26}$

The sampling media used, CapSure ${ }^{\circledR}$ VP, was obtained from Berkshire ${ }^{\circledR}$. The media is $100 \%$ knitted polyester and originally $23 \mathrm{~cm} \times 23 \mathrm{~cm}$ in size. To allow for easy handling while sampling the media was cut to approximately $4.0 \mathrm{~cm}$ x $1.5 \mathrm{~cm}$ (Figure 3.2) swabs using a paper cutter. The swabs were pre-conditioned prior to use by placing and storing them in a glass jar in a laboratory oven at approximately $80^{\circ} \mathrm{C}$.

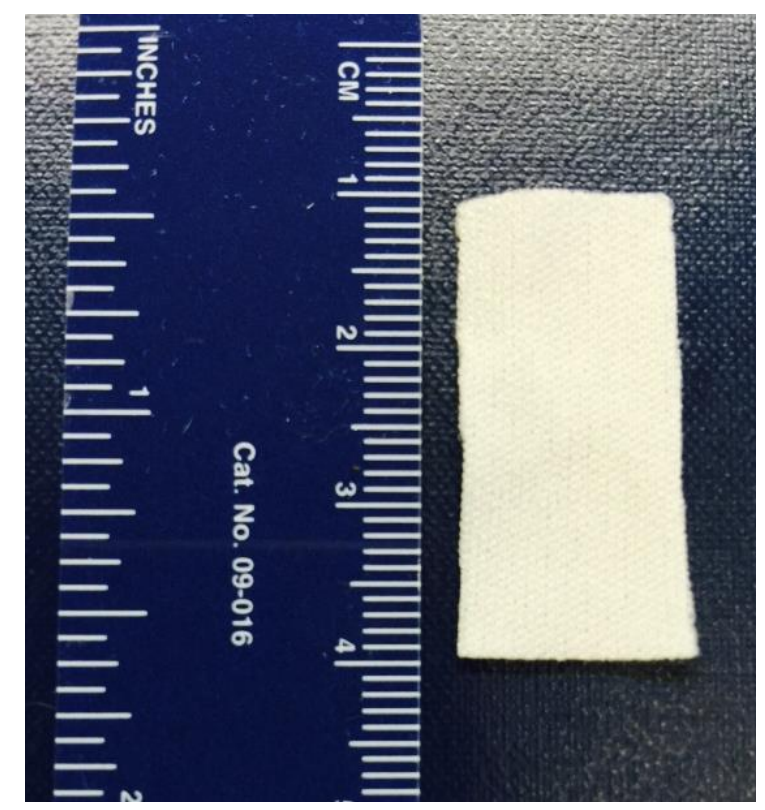

Figure 3.2 Cut CapSure ${ }^{\circledR}$ VP swab measuring approx. 3 x $1.5 \mathrm{~cm}$ used as sampling media.

\subsubsection{Instrumentation}

The instrument used was an Agilent ${ }^{\circledR}$ 7890B gas chromatograph coupled with an Agilent 5977A mass selective detector. Both liquid and solid samples were placed inside an Agilent ${ }^{\circledR}$ "ultra inert" glass microvial and inserted into the thermal separation probe (Figure 3.3A). 
Samples were introduced through thermal desorption by inserting the probe into a thermal separation probe adapter, both commercially available through Agilent® (Agilent Technologies Inc., Santa Clara, CA, USA), affixed to the inlet of the instrument. (Figure 3.3B). Upon desorption samples were swept into the GC column by the carrier gas. The instrument was tuned weekly using a standard autotune and the thermal probe cleaned as needed. O-rings were also replaced on the probes and inlet cap as needed.

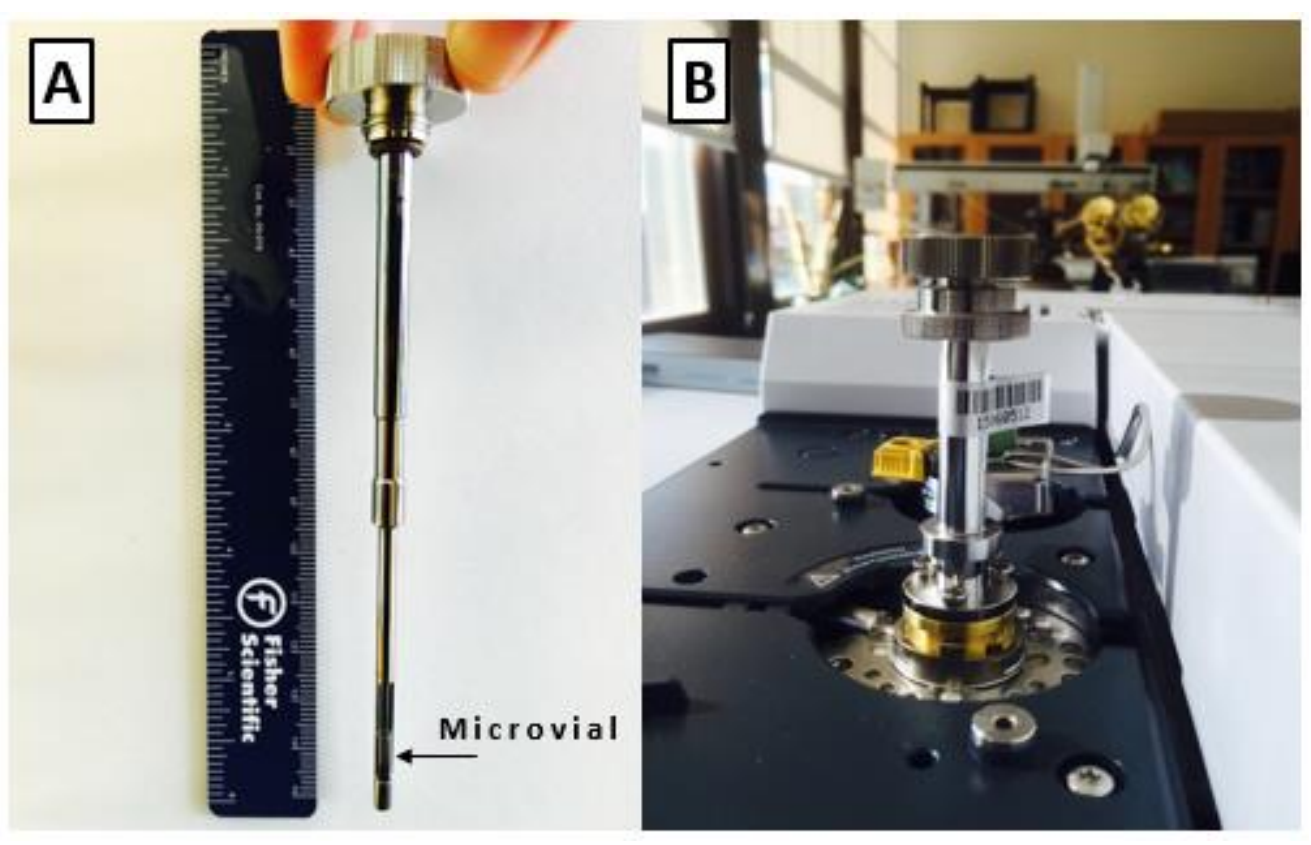

Figure 3.3A Thermal separation probe and sample vial situated in the sample loading apparatus. B. Thermal desorption unit affixed to the inlet of the GC system. Both are commercially available through Agilent Technologies Inc., Santa Clara, CA, USA.

The inlet temperature, desorption temperature with respect to this project, was $140{ }^{\circ} \mathrm{C}$. A DB-5MS (30 m x $0.250 \mathrm{~mm} \times 0.50 \mu \mathrm{m})$ Agilent ${ }^{\circledR}$ column was used with helium at a column flow of $1.2 \mathrm{~mL} / \mathrm{min}$. An initial temperature of $30{ }^{\circ} \mathrm{C}$ was held for 2 minutes then ramped to 300 ${ }^{\circ} \mathrm{C}$ at $15{ }^{\circ} \mathrm{C}$ per minute and held for 0.25 minutes. MS source and quadrupole temperatures were $230{ }^{\circ} \mathrm{C}$ and $150{ }^{\circ} \mathrm{C}$, respectively. Scan and selected ion monitoring (SIM) methods were both used for compound detection and identification as described below. 


\subsubsection{Authentic FDR Samples}

Collection of control shooting samples was accomplished by firing a designated number of shots (1-5) from a firearm then wiping both the left and right hands of the shooter with a prewetted swab (WVU IRB protocol \#1209000337). Test shootings were carried out during two shooting sessions using a Smith and Wesson 0.38 revolver and a Glock $9 \mathrm{~mm}$ semiautomatic pistol. The swabs were pre-wet with $\sim 1 \mathrm{~mL}$ of isopropyl alcohol prior to thorough wiping of the top and palm of the hand and the crease between the index finger and thumb. Both hands were sampled on the same swab which was then placed in a glass tube, capped, and labeled.

Succession samples, ranging from 1-5 shots, were collected during an additional shooting session using an in-house fabricated swab holder, depicted in Figure 3.4, to minimize the swab sampling surface area $\left(\sim 1.5 \mathrm{~cm}^{2}\right)$ to concentrate the collected residues in as small a surface area as feasible. Control background samples from the skin, hand blanks were also collected, using the same sampling method as mentioned above. These samples were collected from the same subject that provided the shooting samples.

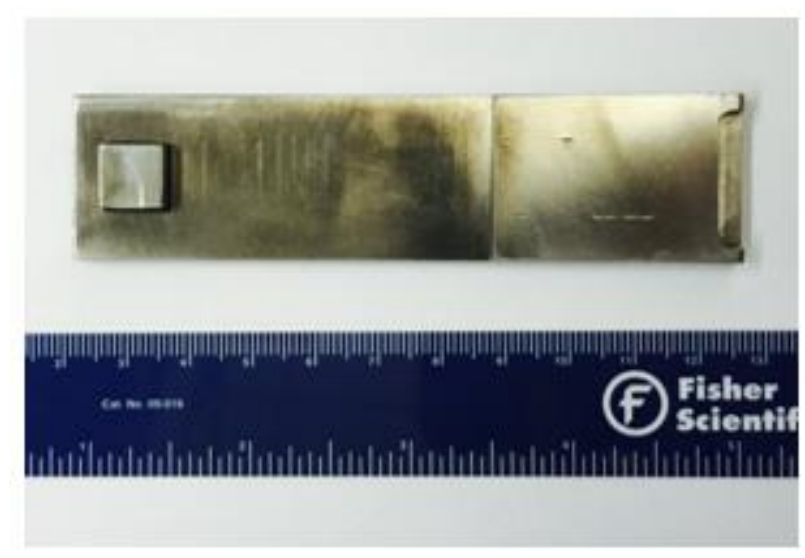

Figure 3.4 In-house fabricated swab holder used for authentic sample collection. 


\subsection{Results and Discussion}

\subsubsection{Compound Selection}

Thirteen compounds previously identified in literature as OGSR were analyzed initially using a scan method to obtain relative retention times. In addition, experiments analyzing positive shooting swabs were performed to identify potential compounds. It was important to collect post-firing FDR rather than materials from unburnt propellants to insure that the final target list was realistic and representative of authentic firing events. Note that neither nitroglycerine nor n-nitrosodiphenylamine were selected as target compounds due to anticipated thermal degradation. ${ }^{11,27}$ The final target list contained DPA, 2-NDPA, 4-NDPA, MC, EC, 2,4DNT, DBP, DMP, carbazole and the EPA 529 surrogate which contained two compounds (Table 3.1).

Table 3.1 Final compound target list with corresponding abbreviations and ions used in the developed SIM method where the quantifier ion is the most intense ion.

\begin{tabular}{|c|c|c|c|c|c|}
\hline \multirow{2}{*}{ COMPOUND } & \multirow{2}{*}{ ABBR. } & \multirow{2}{*}{$\begin{array}{c}\text { Rt } \\
(\mathbf{m i n})\end{array}$} & \multirow{2}{*}{$\begin{array}{c}\text { SIM } \\
\text { WINDOW }\end{array}$} & \multicolumn{2}{|c|}{$\begin{array}{c}\text { SELECTED IONS } \\
(\mathrm{M} / \mathrm{Z}) \\
\end{array}$} \\
\hline & & & & $\begin{array}{l}\text { Quantifier } \\
\text { Ion }\end{array}$ & $\begin{array}{l}\text { Qualifier } \\
\text { Ion }\end{array}$ \\
\hline EPA SURROGATE & EPA & $11.95,13.13$ & $3 \& 4$ & 91.1 & 148.1 \\
\hline DIMETHYL PHTHALATE & DMP & 13.29 & 5 & 163.1 & 92.1 \\
\hline 2,4-DINITROTOLUENE & 2,4-DNT & 14.05 & 6 & 165.1 & 89.1 \\
\hline DIPHENYLAMINE & DPA & 14.83 & 7 & 169.2 & 83.6 \\
\hline METHYL CENTRALITE & $\mathrm{MC}$ & 16.47 & 8 & 134.1 & 106.1 \\
\hline CARBAZOLE & Carb & 16.61 & 9 & 167.2 & 139.1 \\
\hline ETHYL CENTRALITE & $\mathrm{EC}$ & 16.81 & 10 & 148.2 & 268.2 \\
\hline DIBUTYL PHTHALATE & DBP & 17.11 & 11 & 149.1 & 150.1 \\
\hline
\end{tabular}




\begin{tabular}{|c|c|c|c|c|c|}
\hline 2-NITRODIPHENYLAMINE & 2-NDPA & 17.40 & 12 & 167.2 & 214.1 \\
\hline 4-NITRODIPHENYLAMINE & 4-NDPA & 19.26 & 13 & 167.1 & 214.1 \\
\hline
\end{tabular}

\subsubsection{Instrumentation and Procedure Optimization}

The most critical experimental parameter to optimize was the inlet temperature. An inlet temperature of $200{ }^{\circ} \mathrm{C}$ or higher is common for OGSR compounds extracted via solid phase microextraction, where little to no interference is expected from the fiber. Here, extraction of OGSR compounds from hand swabs is occurring directly in the GC inlet and interferences from the swabs and hands are inevitably produced. Thus, optimization of the inlet temperature required balancing optimal recovery of target analytes against minimization of background interferences from swabs and hands. As a result of optimization experiments, an inlet temperature of $140{ }^{\circ} \mathrm{C}$ was selected along with a 2 minute initial oven temperature hold at $30{ }^{\circ} \mathrm{C}$.

To further decrease background expected from the swabs and hands and to increase sensitivity a SIM method was employed. For each compound, a target and one qualifier ion were selected based on intensity. Dwell windows for each compound were optimized individually.

\subsubsection{Figures of Merit}

As this study was intended for proof-of-concept purposes, only a partial validation study was done. Detection limits were estimated based on signal-to-noise ratios. Signal to noise ratios $(\mathrm{S} / \mathrm{N})$ were calculated using the MassHunter ${ }^{\circledR}$ software for each compound window for each ion (12 windows, 2 ions per window) using 3 blank swabs. The signal measurement was a function of height and the noise measurement was calculated as the root mean square (RMS) $\mathrm{x} 3$, in which a range within the individual compound window was selected as the noise region. The max $\mathrm{S} / \mathrm{N}$ value between the 3 blank swabs was designated as the $\mathrm{S} / \mathrm{N}$ for that ion in that specific compound window. Data was collected from swab spikes (method LOD) as well as for direct 
spikes into the microvial (no swab, instrument LOD). Both method and instrument LOD results can be seen in Table 3.2. Repeatability was analyzed by spiking $100 \mathrm{ng}$ of all compounds in a vial $(n=5)$ in which repeatability of retention times was $<0.60 \%$ for all ions whereas the $\%$ RSD of ion peak heights was much higher with a minimum of $20 \%$. Compound ion ratios provided lower $\%$ RSDs ranging from $7 \%$ to $57 \%$. As a result, acceptable ion ratios were calculated and ranges were selected for each compound individually.

Table 3.2 Instrument and method LOD values for target compounds.

\begin{tabular}{|c|c|c|}
\hline & Instrument (vial) & Method (swab) \\
\hline DMP & $0.5 \mathrm{ng}$ & $1 \mathrm{ng}$ \\
\hline $\mathbf{2 , 4 - D N T}$ & $1 \mathrm{ng}$ & $500 \mathrm{ng}$ \\
\hline DPA & $0.5 \mathrm{ng}$ & $5 \mathrm{ng}$ \\
\hline MC & $0.05 \mathrm{ng}$ & $5 \mathrm{ng}$ \\
\hline Carb & $0.05 \mathrm{ng}$ & $100 \mathrm{ng}$ \\
\hline EC & $0.05 \mathrm{ng}$ & $0.05 \mathrm{ng}$ \\
\hline DBP & $0.05 \mathrm{ng}$ & $0.05 \mathrm{ng}$ \\
\hline 2-NDPA & $0.05 \mathrm{ng}$ & $20 \mathrm{ng}$ \\
\hline 4-NDPA & $20 \mathrm{ng}$ & $500 \mathrm{ng}$ \\
\hline
\end{tabular}

\subsubsection{Application to Authentic FDR Samples}

A flowchart, Figure B3.1 (Appendix B), was developed and criteria established to determine the presence of OGSR compounds on authentic shooting samples. This method was utilized to differentiate the OGSR compounds from the interferences in the background of the swabs and hand and incorporated retention time and ion abundance information. Specifically, the flowchart depicts the 4 criteria that were applied to the peaks of interest in the shooting chromatograms; retention times (Rt) of both ions, ion presence, ion ratio, and $\mathrm{S} / \mathrm{N}$ ratio. Rt and 
ion ratio values were established through the use of standards for each compound with \%RSD values ranging from $0.08-0.20 \%$ for $\mathrm{Rt}$ and $8-54 \%$ for ion ratio. Table B3.1 summarizes \%RSD data for all compounds. Two sets of ranges for each of these criteria were created by applying \pm 1 and \pm 2 standard deviations to the average values. The criteria for DPA seen in Table B3.2 serves as an example. If all four criteria were met, the sample was considered to be positive for the presence of that OGSR compound for purposes of this study.

Sixteen authentic shooting samples from the initial shooting session were analyzed. The collected sample swabs were too large to fit into appropriate microvials and therefore had to be cut prior to being analyzed. The first swab analyzed was cut in half and was difficult to place inside the glass microvial for fear of shattering the vial, because of this the remaining swabs (15) were cut into three pieces; 11 of the 15 were analyzed completely and 4 of the 15 cut only the middles were analyzed, for a total of 39 analyzed samples. Having to cut the swabs afforded an opportunity to evaluate the homogeneity of the residue as collected across the swab. Out of the 12 swabs that were analyzed completely there was only one instance in which both side sections contained a compound (EC) in which the middle did not and 2 instances in which one side contained a compound (2-NDPA) that the middle did not. It is worth mentioning that although EC was only detected on the sides of the one swab, DPA was only detected on the middle of that swab. In fact, $50 \%$ of the swabs middle sections contained 1-3 compounds more than detected on the side pieces; $33 \%$ of the swabs were homogeneous across the entire swab. These results indicate that although the middle of the swab provides great detail the sides cannot be overlooked. For future work the size of the swab should be reduced or ran in its entirety.

The first set of positive control swabs were collected from shooters who had discharged 5 shots. On each of these 16 swabs, 3-5 target compounds were detected. Sample data for two 
authentic shooting samples for DPA can be seen in Table 3.3. Overall, DBP, EC, DMP, and DPA were detected on $88-100 \%$ of the swabs analyzed. None of the swabs had detectable levels of 4-NDPA, 2,4-DNT, MC and carbazole, and 2-NDPA was detected on less than $50 \%$ of the collected samples. These results are summarized in the first row of Table 3.4. Given the relative success of this analysis, successive samples of 1-5 shots were collected and analyzed in triplicate with the exception of 4 and 5 shots in which only one swab was collected. This was an oversight that was not discovered in time to address for this project. A swab holder, described and depicted in the experimental section of this article, was used to pre-concentrate the sample by minimizing the surface area subject to sampling. Again, the collected samples had to be cut prior to being analyzed. EC and DPA which were detected in the original 5 shot samples were seen in 2 and even 1 shot samples although what was detected varied from sample to sample (Table 3.4). As seen in the table, no compounds were detected at 4 shots, this could be due to only one swab being collected and analyzed. In addition to only one swab being collected, an instrument communication error led to the data of one of the two halves to not be collected as was the case for a 2 shot sample.

Table 3.3 Example authentic shooting sample data for DPA including the 3 of the 4 criteria applied to the peaks of interest; ion presence, ion ratio, and S/N. All retention times for both base ion (169.2) and qualifier ion (83.6) were 14.84 minutes.

\begin{tabular}{|l|c|c|c|c|c|}
\hline \multicolumn{1}{|c|}{ SHOOTER FILE } & $\begin{array}{c}\text { BOTH IONS } \\
\text { PRESENT }\end{array}$ & $\begin{array}{c}\text { QUANTIFIER } \\
\text { PEAK ION } \\
\text { HEIGHT }\end{array}$ & $\begin{array}{c}\text { QUALIFIER } \\
\text { ION } \\
\text { HEIGHT }\end{array}$ & RATIO \% & $\begin{array}{c}\text { QUANTIFIER \& } \\
\text { QUALIFIER } \\
\text { PEAK S/N }\end{array}$ \\
\hline $\begin{array}{l}\text { Shooting Swab 1 } \\
\text { piece 1 }\end{array}$ & Yes & $2.16 \times 10^{4}$ & $3.12 \times 10^{3}$ & 14.5 & Above \\
\hline $\begin{array}{l}\text { Shooting Swab 1 } \\
\text { piece 2 }\end{array}$ & Yes & $3.79 \times 10^{4}$ & $6.31 \times 10^{3}$ & 16.7 & Above \\
\hline $\begin{array}{l}\text { Shooting Swab 1 } \\
\text { piece 3 }\end{array}$ & Yes & $7.05 \times 10^{4}$ & $1.25 \times 10^{4}$ & 17.7 & Above \\
\hline $\begin{array}{l}\text { Shooting Swab 2 } \\
\text { piece 1 }\end{array}$ & Yes & $3.34 \times 10^{4}$ & $6.53 \times 10^{3}$ & 19.6 & Above \\
\hline
\end{tabular}




\begin{tabular}{|l|c|c|c|c|c|}
\hline $\begin{array}{l}\text { Shooting Swab 2 } \\
\text { piece 2 }\end{array}$ & Yes & $3.58 \times 10^{4}$ & $5.34 \times 10^{3}$ & 14.9 & Above \\
\hline $\begin{array}{l}\text { Shooting Swab 2 } \\
\text { piece 3 }\end{array}$ & Yes & $1.27 \times 10^{5}$ & $2.10 \times 10^{4}$ & 16.6 & Above \\
\hline
\end{tabular}

Table 3.4 Overall authentic shooting sample results. Values are by overall swab not by piece.

\begin{tabular}{|c|c|c|c|c|c|c|}
\hline & \# SHOTS & DMP & DPA & EC & DBP & 2-NDPA \\
\hline $\begin{array}{c}\text { FIRST SESSION: } \\
\text { NO HOLDER }\end{array}$ & 5 Shots & 14 of 16 & 14 of 16 & 16 of 16 & 16 of 16 & 7 of 16 \\
\hline \multirow{4}{*}{$\begin{array}{c}\text { SECOND } \\
\text { SESSION: }\end{array}$} & 1 Shot & 3 of 3 & 1 of 3 & 2 of 3 & 3 of 3 & $\mathrm{x}$ \\
\cline { 2 - 7 } & 2 Shots & 3 of 3 & 1 of 3 & 3 of 3 & 3 of 3 & $\mathrm{x}$ \\
\cline { 2 - 7 } & 3 Shots & 3 of 3 & $\mathrm{x}$ & 2 of 3 & 3 of 3 & $\mathrm{x}$ \\
\cline { 2 - 7 } & 4 Shots & $\mathrm{x}$ & $\mathrm{x}$ & $\mathrm{x}$ & $\mathrm{x}$ & $\mathrm{x}$ \\
\hline
\end{tabular}

Three blank swabs and 16 hand blank samples were collected and analyzed and the data interpreted as described with the flowchart method. DMP and DBP were found in both the blank swabs and hand blank samples with an occurrence of $84 \%$ and $95 \%$ respectively. Phthalates are used in products such as plastics, cosmetics, insect repellants and pesticides; thus the likelihood of these products being found in the background was substantial and expected. Therefore, for this study, DMP and DBP were eliminated as FDR target compounds. Surprisingly, EC was found in $26 \%$ of the background samples but DPA was not detected in any background swabs. There are two possible explanations - true contamination/transfer or a false positive. The latter could be addressed using additional qualifier ions.

The data showed that in this study EC is the best marker of OGSR in this assay, followed by DPA. EC was confirmed in the majority of shooter swabs including those obtained after firing a single shot. However, because EC was seen on a significant number of blanks, this 
finding must be interpreted with caution. One compound, 2-NDPA is found in less than $40 \%$ of the known shooter swabs across 5 shot samples. This does not mean that this compound should not be monitored, but that when it is found, it adds to the weight of the evidence that FDR is present. Finally, it is worth noting that DPA and 2-NDPA were present only on swabs where EC was also present.

\subsubsection{Use of Surrogates and Statistical Interpretation of Results}

For OGSR to be useful in forensic practice, statistically-based data analysis and interpretation methods need to be developed. The dataset described here is too small to allow for any conclusions to be drawn, but it is sufficient to propose a data analysis approach that integrates retention time and ion ratio data. The methodology would be applicable to GC/MS (SIM) as well as to previously reported $\mathrm{LC} / \mathrm{MS}^{\mathrm{n}}$ procedures in which multiple ion-selected ion monitoring (SRM/MRM) is used. The properties of a molecule that influence retention time on a GC column such as used here (molecular weight, polarity, vapor pressure, etc.) are different (but not completely independent) from characteristics that dictate molecular fragmentation and resulting ion ratio. As such, probabilities associated with each can be combined. Here, this information was used in two ways: first, use of the results from the surrogate standards to establish acceptance criteria for retention time and ion ratios; and second, to provide a probabilistic framework for interpreting results as positive/negative for OGSR. Because the surrogates were added to all samples, a much larger data set collected over time (here, several weeks under the same conditions) is available and provides a measure of reproducibility (as opposed to repeatability). There are existing guidelines for ion ratio criteria (i.e., $20 \%$ for ion ratios ${ }^{28,29}$ ) but none of these appear to have an empirical foundation. Therefore, an empirical approach using the surrogate standards is a reasonable alternative. 
For this work, a method was used that is an adaptation of recently published work by Woldegebriel, et. al. ${ }^{30,31}$ in which reference standards are used to generate probabilities from bivariate probability density functions. The results for the surrogate standards $(n=71)$ are shown in Figures 3.5A and B. The histograms for the retention time are shown on the bottom axis and the vertical axis represents the distribution of the ion ratio. The density plot is based on a kernel density non-parametric fit with 50\%, 90\%, 95\%, and 99\% Mahalanobis distance ellipses overlaid. Using this criteria, one outlier is noted in the dataset of surrogate 1 and 4 for surrogate 2, all based on ion ratio. One sample, a hand blank, was outside the $95 \%$ ellipse for both compounds; for surrogate 2 , the point on the upper left side outside the ellipse is for the same sample. The other three outside of this ellipse of surrogate 2 consist of one hand swab blank and two from the five-shot series.

In practice, the surrogate criteria could be used as a qualifier for data acceptance; if the RT/ratio pair for any given sample falls outside of the $95 \%$ ellipse, this suggests a procedural or recovery problem that would have to be considered in evaluating the results for that hand swab. As more historical data is gathered from these assays, it will be possible to fit bivariate probability distributions to retention time and ion ratio data which could allow for additional statistical or Bayesian evaluations. ${ }^{30-32}$

The findings described in Section 3.3 showed that the phthalates are not reliable markers of OGSR and are thus not considered here. For the remaining compounds that were observed in shooter samples, contour plots were prepared the same way using the same criteria data used to establish the ranges used in Section 3.3. These are provided in Appendix B (Figure B3.2a-c) with the $95 \%$ region indicated with a box. These plots were used to establish the $95 \%$ 
confidence thresholds that would be used to classify a combined retention time and ion ratio as originating from a reference target compound.

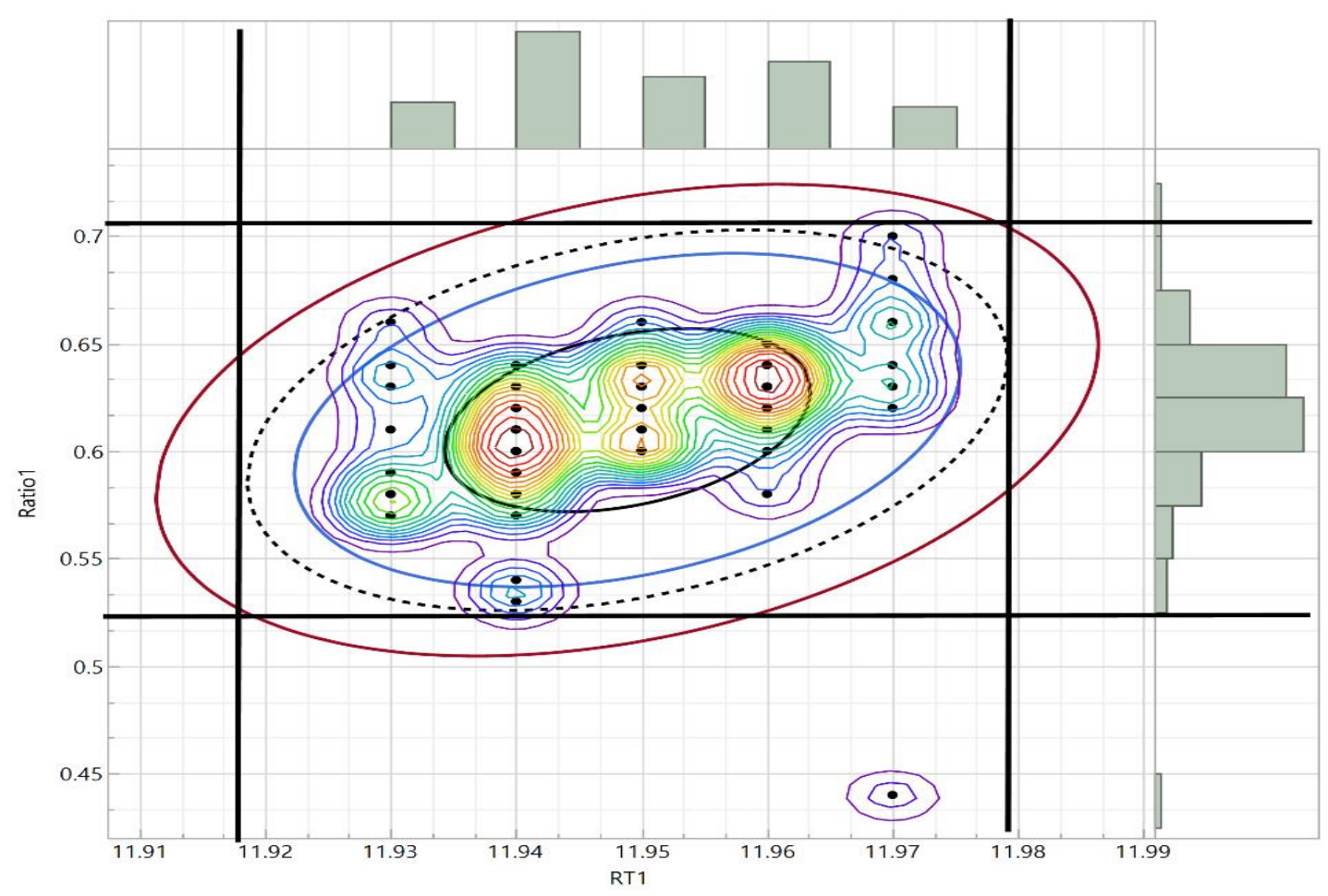

Figure 3.5A Surrogate compound 1, bivariate plot with histograms $(\mathrm{n}=70)$. 


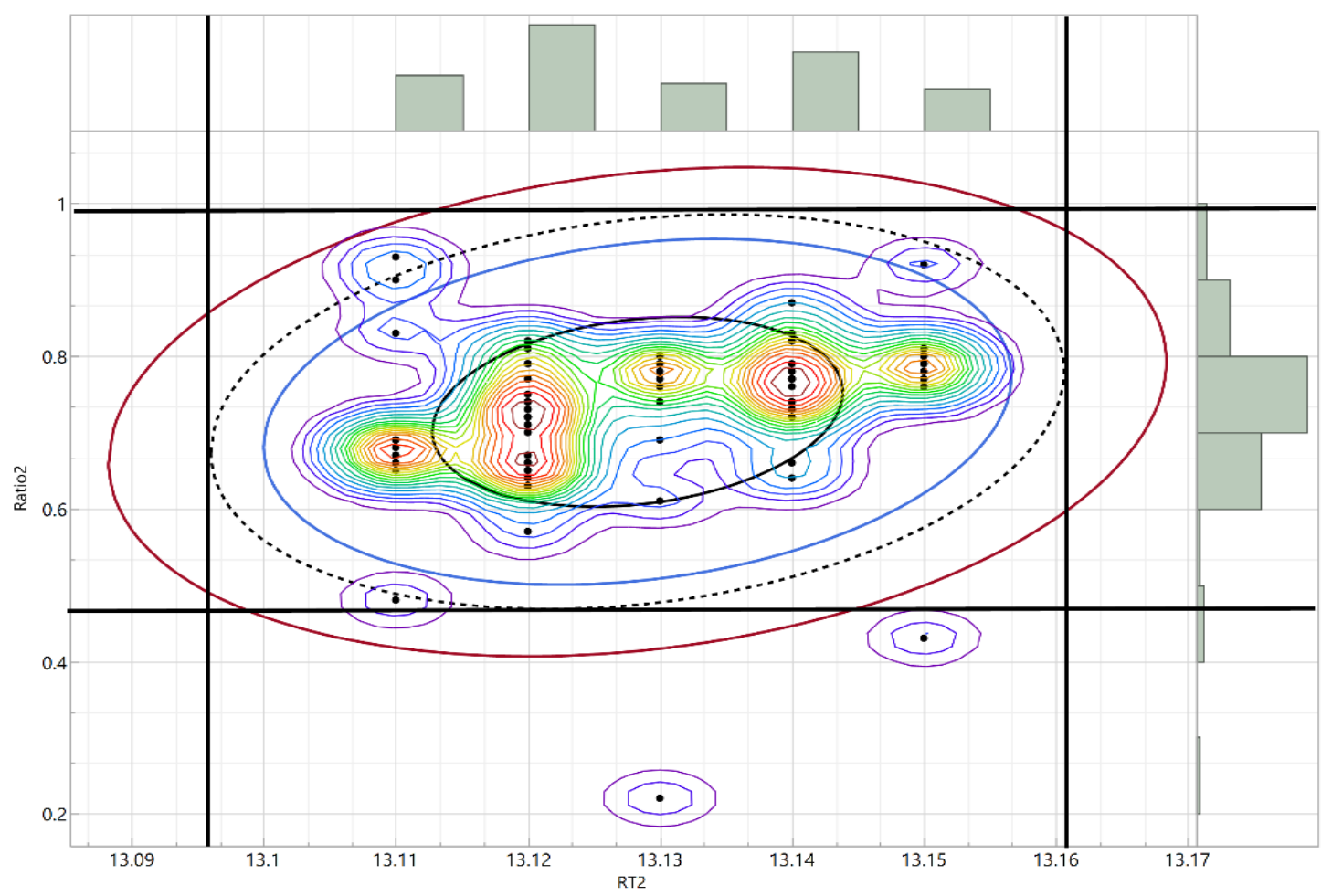

Figure 3.5B Surrogate compound 2, bivariate plot with histograms $(n=70)$.

The results obtained via flowchart and plot methods, for all shooting swabs pieces analyzed $(n=59)$, are compared in Table 3.5. Overall, the presence of two out of the three compounds compared was increased when analyzed via plots. Differences in the results were investigated and although the percent presence for DPA for both methods is the same, the results of 6 swabs were different. Three swabs were rejected via the flowchart method for the presence of DPA based on the qualifier retention ions, which were not taken into account for the plots. The three remaining swabs were included in flowchart results for the presence of DPA based on the ratio falling in the range established from 2 standard deviations. These ratio values reside just outside of the plots established ranges, with ratios of $30.6 \%, 31.4 \%$, and $31.7 \%$. The difference in the EC results was based on a ratio value excluding a swab in the flowchart method which was also the case for one of the 2-NDPA swabs. The final difference seen in the 2-NDPA results was 
due to a swab being excluded because the height for the base peak ion fell below the established S/N. Overall, both methods provided advantages and disadvantages; the flowchart method although tedious and very time consuming took more variables into consideration such as the qualifier ion retention time and $\mathrm{S} / \mathrm{N}$ and the plots, unlike the flowchart, provide a justifiable means to identify outliers and provide a confidence level in identifying the presence of compounds in shooting swabs.

Table 3.5 Results for all pieces of shooting swabs when analyzed via the contour plots established from criteria data for each compound compared to flowchart results. $(n=59)$

\begin{tabular}{|c|c|c|c|c|}
\hline COMPOUND & $\begin{array}{c}\text { \% PRESENCE } \\
\text { FLOWCHART }\end{array}$ & $\begin{array}{c}\text { \% PRESENCE } \\
\text { PLOTS }\end{array}$ & $\begin{array}{c}\# \\
\text { DIFFERENT }\end{array}$ & $\begin{array}{c}\# \\
\text { SHOTS }\end{array}$ \\
\hline EC & $80 \%$ & $81 \%$ & 1 & $1-5$ \\
\hline DPA & $56 \%$ & $56 \%$ & 6 & $2,3,5$ \\
\hline 2-NDPA & $14 \%$ & $17 \%$ & 2 & 5 \\
\hline
\end{tabular}

\subsection{Conclusions}

To be of practical use in forensic scenarios, any proposed assay for OGSR should be capable of detecting the residue associated with 1-3 shots. The thermal desorption GC/MS system described here shows promise in this regard, but addition work remains before the technique can be fairly evaluated. For example, detection of EC in blanks is a concern; adoption of additional qualifier ions across all of the target compounds will be essential. The other obvious limitation is the way in which the swab is loaded into the TSP. For this study, the sampling media must be inserted into a glass microvial which results in very tight packing that inevitably impacts gas flow and efficient heating. Variations in the design, such as developing a microvial with a series of holes or by eliminating the microvial and inserting the swab directly into the probe are options. The advantages of being able to use GC/MS this way (no sample 
preparation, no pre-concentration, and availability of instrumentation) argues for this type of continued investigation which is currently underway in our laboratory.

Despite the identified limitations, the method presented here appears capable of detecting OGSR compounds at forensically relevant concentrations. The surrogate compounds, characterized by the bivariate plots provided means to assign probabilities to combinations of ion ratios and retention time data and to establish acceptance criteria. This same method is also useful for interpreting analytical results in a way that avoids checklist/flowcharts while providing probabilities derived from a long-term data set.

\subsection{Funding and Additional Notes}

This work was supported by the National Institute of Justice through the Forensic Center of Excellence at RTI International, contract \# 6-321-0213168. The TSP and GC/MS were supplied by Agilent Technologies.

Approval for human subject sampling was obtained through the West Virginia University Institutional Review Board (IRB), protocol 1209000337. 


\subsection{References}

1. J. W. Moran and S. Bell, Analytical Chemistry, 2014, 86, 6071-6079.

2. O. Dalby, D. Butler and J. W. Birkett, J. Forensic Sci., 2010, 55, 924-943.

3. F. S. Romolo and P. Margot, Forensic Sci.Int., 2001, 119, 195-211.

4. A.-L. Gassner and C. Weyermann, Forensic Sci.Int., 2016, 264, 47-55.

5. R. V. Taudte, C. Roux, L. Blanes, M. Horder, K. P. Kirkbride and A. Beavis, Analytical and Bioanalytical Chemistry, 2016, 408, 2567-2576.

6. ASTM-International, ASTM E1588-10e1: Standard Guide for Gunshot Residue Analysis by Scanning Electron Microscopy/ Energy Dispersive X-ray Spectrometry, https://www.astm.org/cgi-bin/resolver.cgi?E1588-16a).

7. Z. Oommen and S. M. Pierce, J. Forensic Sci., 2006, 51, 509-519.

8. J. Arndt, S. Bell, L. Crookshanks, M. Lovejoy, C. Oleska, T. Tulley and D. Wolfe, Forensic Sci.Int., 2012, 222, 137-145.

9. J. Moran and S. Bell, Int. J. Ion Mobil. Spec., 2013, 16, 247-258.

10. M. X. Zhao, S. C. Zhang, C. D. Yang, Y. C. Xu, Y. X. Wen, L. S. Sun and X. R. Zhang, J. Forensic Sci., 2008, 53, 807-811.

11. O. Dalby and J. W. Birkett, Journal of Chromatography A, 2010, 1217, 7183-7188.

12. C. West, G. Baron and J. J. Minet, Forensic Sci.Int., 2007, 166, 91-101.

13. D. Muller, A. Levy, A. Vinokurov, M. Ravreby, R. Shelef, E. Wolf, B. Eldar and B. Glattstein, J. Forensic Sci., 2007, 52, 75-78.

14. G. L. Burleson, B. Gonzalez, K. Simons and J. C. C. Yu, Journal of Chromatography A, 2009, 1216, 4679-4683.

15. R. V. Taudte, A. Beavis, L. Blanes, N. Cole, P. Doble and C. Roux, BioMed research international, 2014, 2014, 965403-965403.

16. C. Weyermann, V. Belaud, F. Riva and F. S. Romolo, For. Sci Int., 2009, 186, 29-35.

17. J. Andrasko and S. Stahling, J. Forensic Sci., 1999, 44, 487-495.

18. J. Andrasko and S. Stahling, J. Forensic Sci., 2003, 48, 307-311.

19. K. H. Chang, C. H. Yew and A. F. L. Abdullah, J. Forensic Sci., 2014, 59, 1100-1108. 
20. M. Gallidabino, F. S. Romolo, K. Bylenga and C. Weyermann, Anal. Chem., 2014, 86, 4471-4478.

21. B. Yeager, K. Bustin, J. Stewart, R. Dross and S. Bell, Analytical Methods, 2015, 7, 9683-9691.

22. S. Bell, Organic Gunshot Residue Analysis for Potential Shooter Determination, https://www.forensiccoe.org/Our-Impact/Advancing-Technology/Reports/OrganicGunshot-Residue-Analysis-for-Potential-Shooter-Determination).

23. S. Bell and L. Seitzinger, Forensic Sci.Int., 2016.

24. A. Tarifa and J. R. Almirall, Sci. Justice, 2015, 55, 168-175.

25. W. Fan and J. Almirall, Analytical and Bioanalytical Chemistry, 2014, 406, 2189-2195.

26. J. W. Munch, Journal, 2002, 1-60.

27. S. Benito, Z. Abrego, A. Sanchez, N. Unceta, M. A. Goicolea and R. J. Barrio, Forensic Sci.Int., 2015, 246, 79-85.

28. T. J. Mahier, I. I. Al-Doush, A. K. Al-Sheikh, M. Al-Tufail and M. J. Bogusz, Accreditation and Quality Assurance, 2010, 15, 659-664.

29. EPA, Washington, DC: U.S Environmental Protection Agency.

30. M. Woldegebriel, Analytical Chemistry, 2015, 87, 11398-11406.

31. M. Woldegebriel, J. Gonsalves, A. van Asten and G. Vivó-Truyols, Analytical Chemistry, 2016, 88, 2421-2430.

32. M. Woldegebriel and G. Vivó-Truyols, Analytical Chemistry, 2015, 87, 7345-7355. 


\section{Chapter 4: Evaluation of host-guest complexation for the analysis of elemental firearm discharge residue with electrospray tandem mass spectrometry (QqQ).}

Analytical limitations and issues with assigning evidentiary value have contributed to the overall decline in the use of FDR as forensic evidence. Coupling the detection of OGSR and GSR would simplify the analytical process while increasing the evidentiary value of FDR. Analytical instruments prevalent in forensic laboratories, such as LC/ESI-MS ${ }^{\mathrm{n}}$ routinely used in toxicology, are typically reserved for the analysis of organic compounds. While methods have been developed for detecting OGSR using LC/ESI-MS ${ }^{\mathrm{n}}$, through complexation, this capability can be extended to inorganic/elemental analysis. Complexation with organic macrocycles such as crown ethers and detection with ESI-MS ${ }^{\mathrm{n}}$ provides a means for dual detection of OGSR and GSR. Prior to studying authentic FDR samples the relative competitiveness of GSR metals with a selected host and complexation relative to a complex GSR system must be understood. An account of the results of the investigations of host-guest complexation with 15-crown-5 and known GSR metals (lead, barium, antimony, and copper) with ESI-MS is presented. Single ligand complexes for lead, barium, and copper and double ligand complexes with lead and barium were identified using isotopic signatures and the presence of bare metal isotopes in corresponding MS/MS spectra. Additionally, binding selectivities, calculated using mass spectral intensities, were estimated and used to establish the overall sequence of preferential binding for the target complexes. Preliminary molecular modeling was used to provide insight and support to the experimental results. Although additional exploration is needed, the combination of ESI-MS ${ }^{\mathrm{n}}$ and complexing agents appears to be a viable technique for the detection of GSR. 


\subsection{Introduction}

Limitations with traditional firearm discharge residue (FDR) detection methods (SEMEDS), along with difficulties associated with evidentiary value has led to the overall decline in the use of FDR evidence. With that, focus has shifted to researching and developing methods for organic gunshot residue (OGSR). Methods applied to OGSR analysis include gas chromatography, high performance liquid chromatography, and capillary electrophoresis all coupled with various detectors. ${ }^{1-3}$ To date, these methodologies have not found widespread use or been implemented in forensic laboratories.

Research has indicated that organic compounds may generally persist longer than inorganic particulates and are less prone to secondary transfer primarily due to their lipophilicity. ${ }^{4}$ While OGSR may appear to be the ideal target of FDR detection for these reasons, limitations still exist. ${ }^{4-7}$ Rather than secondary transfer, OGSR compounds are lost due to evaporation and/or adsorption into the skin. ${ }^{4}$ Although, a combination of organic target compounds may be unique to FDR, individually they have sources other than FDR, as do inorganic particulates. The rates of evaporation and adsorption are compound dependent and thus the compounds and the amounts available for recovery are a function of time. Therefore, careful consideration must be taken when evaluating and interpreting organic results to avoid potential false positives or negatives.

The ability to couple the analysis of GSR and OGSR would simplify the analytical process and aid in result interpretation and increase the evidentiary value of FDR. Ideal methodology would implement instruments currently housed in forensic laboratories such as GC/MS or LC/MS. These instruments are typically reserved for the analysis of organic analytes making the utilization of these instruments ideal for OGSR analysis. On the contrary, elemental 
analysis is traditionally reserved for instruments such as ICP-MS but it is possible with LC/MS when coupled with an electrospray ionization (ESI) ion source. ${ }^{8}$ With recent advances in mass spectrometry, most forensic toxicology laboratories have LC/MS systems with various detectors such as ToF, QToF, QqQ, or other high resolution detectors and the majority of these systems utilize electrospray ionization (ESI). The number of forensic laboratories that have access to electrospray ionization liquid chromatography instruments coupled with $/ \mathrm{MS}^{\mathrm{n}}$ has grown dramatically in facilities that conduct forensic toxicology assays and as such is an ideal platform for modernizing FDR analyses. Certainly many more labs have access to this technology than bulk elemental instrumentation such as ICP-MS.

Since its inception, ESI has found widespread application in studying organic systems including biomolecules such as proteins due to its ability to ionize large molecules with molecular weights of 100,000 Da or more and its characteristic soft ionization. While inorganic and organometallic species have been studied utilizing $\mathrm{ESI}^{9-12}$, some issues have been encountered with elemental electrospray mass spectrometry. ${ }^{8}$ Shou and Browner ${ }^{8}$ describe these problems beginning with the presence of solvent metal clusters over the presence of bare metals in the mass spectra. The soft ionization process, while excellent for studying native state proteins, is a limitation when studying metals in solution phase due to the presence of solvent metal clusters. The harsh collision induced dissociation (CID) conditions required to minimize the number of solvent clusters pose additional concerns. ${ }^{8}$ Furthermore, the metals charge state information is lost under these conditions. ${ }^{8,13,14}$ The final issue is the metal ions lack of hydrophobicity. Shou and Brower ${ }^{8}$ indicates that this hinders the ESI ion evaporation process decreasing the ionization efficiency and thus the sensitivity is lower with elemental ESI-MS relative to ICP-MS and organic ESI-MS. ${ }^{8}$ 
These issues, in part, can be overcome through complexation of the metal ions with supramolecular compounds such as crown ethers. This complexation is referred to as a hostguest interaction or metal-ligand (M-L) interaction. The interactions between host and guest are non-covalent and are typically reversible. ${ }^{15}$ ESI-MS has been used to study host-guest (H-G) interactions and has advantages over traditional methods (potentiometric, spectrophotometric, and NMR) such as reduced sample consumption and analysis times, and the ability to tolerate a variety of solvent conditions and analyze multiple $\mathrm{H}-\mathrm{G}$ complexes in a given sample. ${ }^{16}$ Complexing multiply charged metal ions with supramolecular compounds alleviates the issues described by Shou and Browner ${ }^{8}$. Complexing allows charge state information to be preserved, eliminates the ion evaporation issues caused by to lack of hydrophobicity of metal ions, and simplifies the mass spectra as the complex becomes the dominant species..$^{8,27,28}$ Most importantly, complexation elemental ESI-MS ${ }^{\mathrm{n}}$ analysis allows the relationship between a host structure and guest selectivity to be studied and the determination of a complexes selectivity factors and stability constants and in addition it provides a means for chromatography-free screening for GSR in which confirmation can be achieved by analyzing the isotopic signatures and resulting product ions and. ${ }^{10,16-24}$

Unpublished work completed in our laboratory demonstrated the complexation of crown ether and metals characteristic of GSR (antimony, barium and lead). Crown ethers were discovered as an unanticipated reaction byproduct by Charles J. Pedersen in 1960 and have since been the basis of many $\mathrm{H}-\mathrm{G}$ interaction studies. ${ }^{25}$ The crown ethers oxygen atoms (electron donors) are typically directed outward giving the structure the characteristic crown shape. Upon the addition of a metal ion, a structural change occurs as the result of the electrostatic interaction with the metal ion and the oxygen atoms causing the oxygen atoms to now point inward thus 
"capturing" the metal ion. ${ }^{15}$ This structural change results in a reduction in the diameter of the internal cavity of the crown ether with the inclusion of the metal ion. While the selectivity of complexation isn't completely understood, it is generally accepted that the size of the internal

cavity and the size of the guest ion play vital roles. ${ }^{26}$ Changes in the donor atoms and addition of substituents to the crown ether can provide additional selectivity.

To explore the feasibility of using complexing agents with FDR evidence, a simple crown ether (15-crown-5) was selected for initial experiments. This compound is inexpensive, widely available, and amenable to water/methanol/acetonitrile solvents used with ESI. This crown ether has also been extensively studied and described in the literature. This study utilized ESI tandem mass spectrometry and a variety of scan types to identify complexes and investigate competitive binding with respect to a reference complex to determine relative competitiveness to provide foundational data for final method development and application to GSR. This work is laying the foundation for a single extraction, single instrument method that is feasible and suitable for forensic laboratories.

\subsection{Experimental Section}

\subsubsection{Sample Preparation}

All metal stock standards were single element ICP-MS standards purchased from ULTRA Scientific $®$ (N. Kingstown, Rhode Island) with the exception of potassium which was purchased from SPEX CertiPrep ${ }^{\circledR}$ (Metuchen, New Jersey). 15-crown-5 at 98\% purity was purchased from Sigma-Aldrich ${ }^{\circledR}$ (St. Louis, Missouri). Antimony, barium, copper, and lead stock standard solutions were at a concentration of $10,000 \mu \mathrm{g} / \mathrm{mL}$ in water with dilute nitric acid, while potassium was at $1,000 \mathrm{mg} / \mathrm{L}$ in $2 \%$ nitric acid. A $12,056 \mathrm{ppm}$ stock solution of 15 -crown5 was prepared in HPLC grade methanol (Fisher Chemical, Fair Lawn, New Jersey). The metal 
stock standards and methanol were used without further purification. The stock metal and crown ether solutions were diluted to working solutions of $3.0 \times 10^{-3} \mathrm{M}$ each in methanol. Any additional dilutions were also prepared in the HPLC grade methanol. Other solvents used were acetonitrile and ethyl acetate, LC/MS grade and certified ACS respectively (Fisher Chemical, Fair Lawn, New Jersey).

\subsubsection{Instrumentation}

Experiments were performed on an Applied Biosystems MDS Sciex 3200 QTRAP® triple quadrupole mass spectrometer equipped with an electrospray ion source. Samples were introduced into the source via a syringe pump at a flow rate of $7 \mu \mathrm{L} / \mathrm{min}$. Source parameter conditions were optimized for 15-crown-5. The ESI electrode was maintained at a $5000 \mathrm{~V}$ for all experiments and remaining source parameters can be found in Table 4.1. The parameters TEM and GS1 are set at zero because a heated turbo gas was not needed to aid in solvent evaporation. Compound optimization was performed for each metal complex to provide the compound dependent parameter conditions including declustering potential (DP), entrance potential (EP), collision cell entrance potential (CEP), collision energy (CE), and collision cell exit potential (CXP). For complex identification experiments, DP and EP were maintained at $45 \mathrm{~V}$ and $5 \mathrm{~V}$ respectively. A variety of scan techniques, including Q1 (Q1 MS), Q1 Multiple Ion (Q1MI), Product Ion (MS2) and Multiple Reaction Monitoring (MRM), were utilized in these experiments. For product ion experiments the collision gas (CAD) was maintained at a medium pressure and high pressure for MRM experiments. 
Table 4.1 Electrospray ion source conditions for all mass spectrometry experiments.

\begin{tabular}{rc}
\hline & Condition \\
\hline Curtain Gas (CUR) & $20 \mathrm{psi}$ \\
IonSpray Voltage (IS) & $5000 \mathrm{~V}$ \\
Temperature (TEM) & $0{ }^{\circ} \mathrm{C}$ \\
Gas 1 (GS1) & $10.0 \mathrm{psi}$ \\
Gas 2 (GS2) & $0 \mathrm{psi}$ \\
Interface Heater (ihe) & On \\
\hline
\end{tabular}

The 3200 QTRAP® instrument was tuned and calibrated as per manufacturer recommendations with a positive PPG solution prior to performing experiments. The unit resolution for the 3200 QTRAP® is +/- 0.7 Da. During initials studies peaks associated with potassium $(m / z, 259)$ and sodium $(m / z, 243)$ 15-crown-5 complexes were observed in mass spectra. Sample preparation glassware and sample storage vials were replaced with plastic to reduce the leaching of trace metals into the sample. In addition, carry-over of potassium complexes was observed requiring a rigorous clean cycle to be performed between analyzing samples. This clean cycle consisted of rinsing the syringe and flushing the Peek tubing and source with approximately $1 \mathrm{~mL}$ of ethyl acetate, acetonitrile, and methanol or just the latter two solvents. Upon completion of this clean cycle, background signal of the potassium complexes was reduced to approximately $10^{4}, 1-2$ orders of magnitude lower than the average signal $\left(10^{5}-\right.$ $10^{6}$ ). Solvent background spectra were also collected. 


\subsection{Results and Discussion}

\subsubsection{Complex Identification}

This study first investigated the formation of 15 -crown-5 complexes with antimony, barium, copper, lead, and potassium. The potassium complex was used as a reference based on its high affinity for crown ether and its established history of application in literature. ${ }^{17,18,20,29}$ Target metal solutions were combined in a 1:1 ratio with 15-crown-5 $\left(\mathrm{C}_{10} \mathrm{H}_{20} \mathrm{O}_{5}\right)$ resulting in the crown ether and the metal being at concentrations of $1.5 \times 10^{-3} \mathrm{M}$ each. These mixtures were then diluted by a factor of 10 prior to analysis. A Q1 scan with a range of 50-1000 Da was performed for each sample. Resulting spectra were analyzed for peaks with the correct isotopic signature associated with each metal. The atomic mass and natural isotopic abundance of each isotope of the metals of interest in this study (Table 4.2) aided in the determination of the isotopic signature and calculation of $\mathrm{m} / \mathrm{z}$ values for potential complexes. The peaks identified as metal complexes of interest for barium, copper, and lead can be seen in Figures 4.1 A-F. 
Table 4.2 The atomic masses and natural abundances of each isotope of the metals of interest used to calculate $\mathrm{m} / \mathrm{z}$ values and determine isotopic patterns of the crown ether metal complexes.

\begin{tabular}{|c|c|c|}
\hline Metal & Atomic Mass (amu) & Natural Abundance (\%) \\
\hline${ }^{121} \mathrm{Sb}$ & 120.904 & 57.21 \\
\hline${ }^{123} \mathrm{Sb}$ & 122.904 & 42.79 \\
\hline${ }^{130} \mathrm{Ba}$ & 129.906 & 0.106 \\
\hline${ }^{132} \mathrm{Ba}$ & 131.905 & 0.101 \\
\hline${ }^{134} \mathrm{Ba}$ & 133.905 & 2.417 \\
\hline${ }^{135} \mathrm{Ba}$ & 134.906 & 6.592 \\
\hline${ }^{136} \mathrm{Ba}$ & 135.905 & 7.854 \\
\hline${ }^{137} \mathrm{Ba}$ & 136.906 & 11.232 \\
\hline${ }^{138} \mathrm{Ba}$ & 137.905 & 71.698 \\
\hline${ }^{63} \mathrm{Cu}$ & 62.930 & 69.15 \\
\hline${ }^{65} \mathrm{Cu}$ & 64.928 & 30.85 \\
\hline${ }^{204} \mathrm{~Pb}$ & 203.973 & 1.4 \\
\hline${ }^{206} \mathrm{~Pb}$ & 205.974 & 24.1 \\
\hline${ }^{207} \mathrm{~Pb}$ & 206.976 & 22.1 \\
\hline${ }^{208} \mathrm{~Pb}$ & 207.977 & 52.8 \\
\hline${ }^{39} \mathrm{~K}$ & 38.964 & 93.2581 \\
\hline${ }^{40} \mathrm{~K}$ & 39.964 & 0.0117 \\
\hline${ }^{41} \mathrm{~K}$ & 40.962 & 6.7302 \\
\hline
\end{tabular}

*All values were obtained from The Royal Society of Chemistry’s periodic table of elements. ${ }^{30}$ 


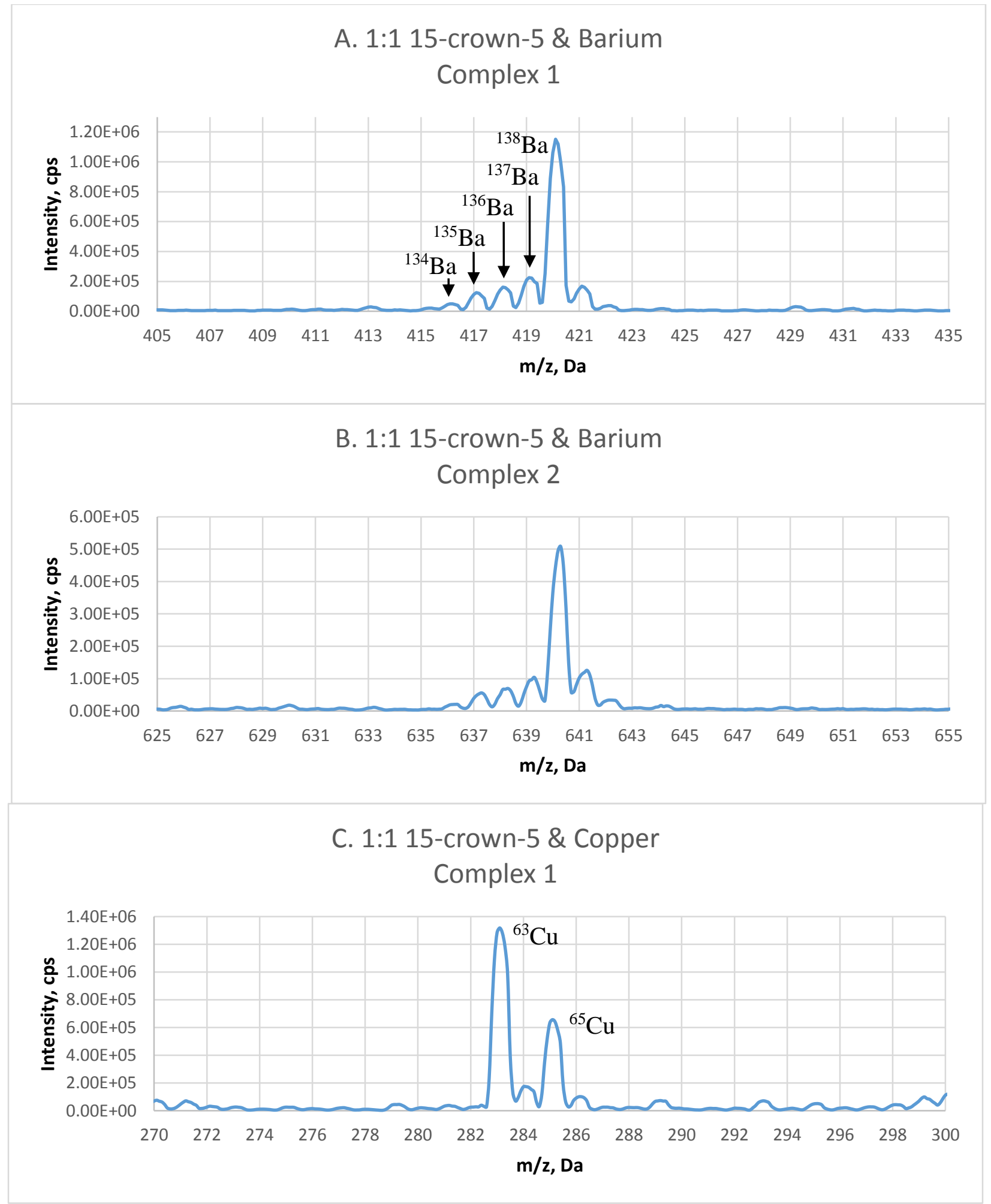

Figure 4.1A-F Peaks identified as having the correct isotopic signature for the metal complexes of interest. The peaks are labeled with the corresponding metal isotope in each Complex 1 spectra. Note - Spectra scan range is from $50-1,000$ Da the figures are zoomed in to the region of interest. Additionally, the $y$-axis (intensity) varies between spectra. 


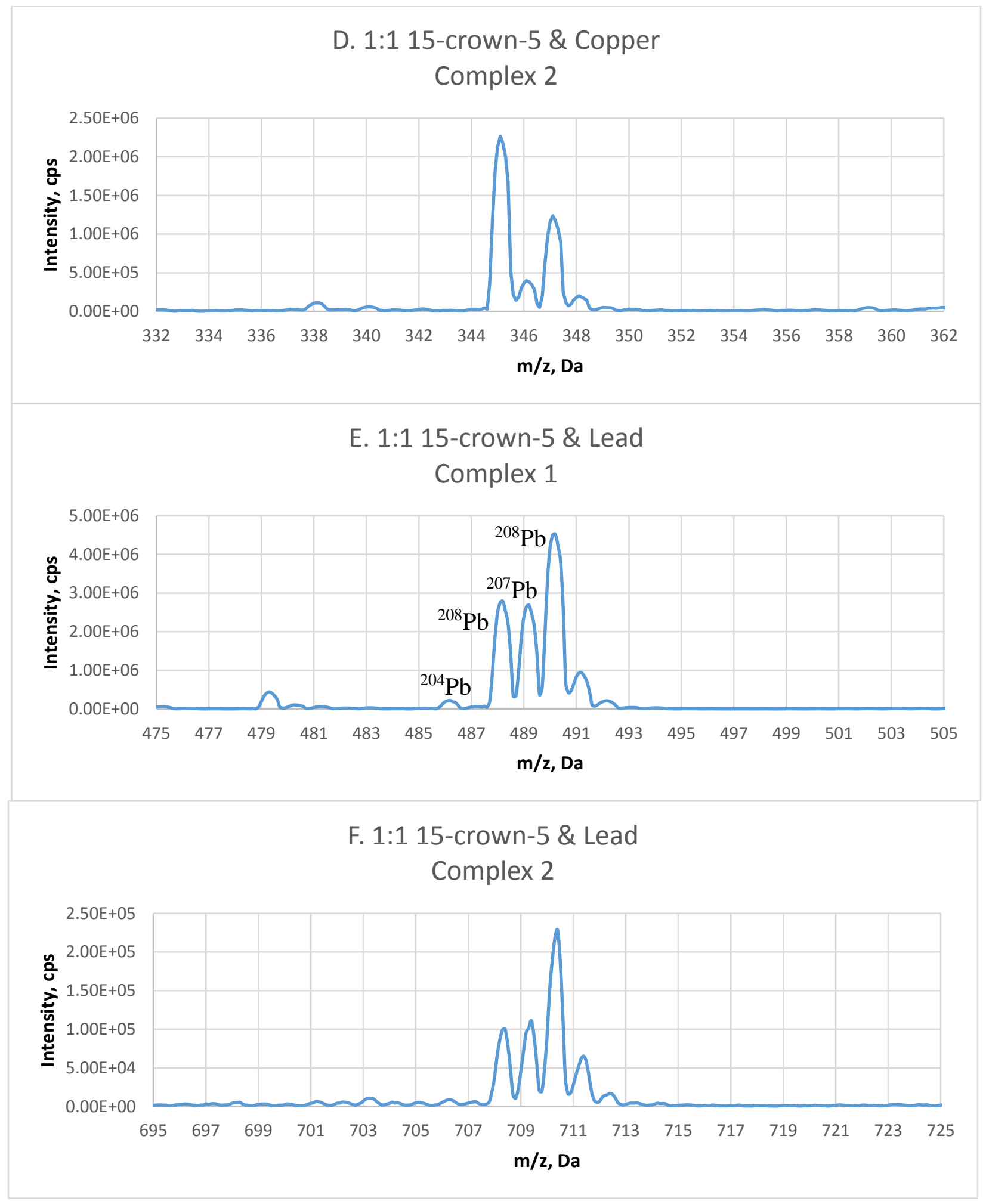

Figure 4.1A-F Continued 
Peak splitting was observed at $m / z 259$ and 479 . These peaks are believed to be that of the potassium complexes. The splitting was thought to be a result of saturation or the formation of multiply charged complex clusters (Appendix C - Figures C4.1 A \& B). A 2:1:1 mixture containing crown ether at $1.5 \times 10^{-3} \mathrm{M}$ and both potassium and lead at $7.5 \times 10^{-4} \mathrm{M}$ each was gradually diluted until the peaks at $\mathrm{m} / z 259$ and 479 showed no signs of splitting. The resulting spectrum was used to confirm the isotopic signature of the potassium complexes (Figure 4.2 A and B). Unfortunately at this concentration other metal complex peaks became undetectable. Therefore, all metal solutions remained at the concentration previously discussed for further experiments.

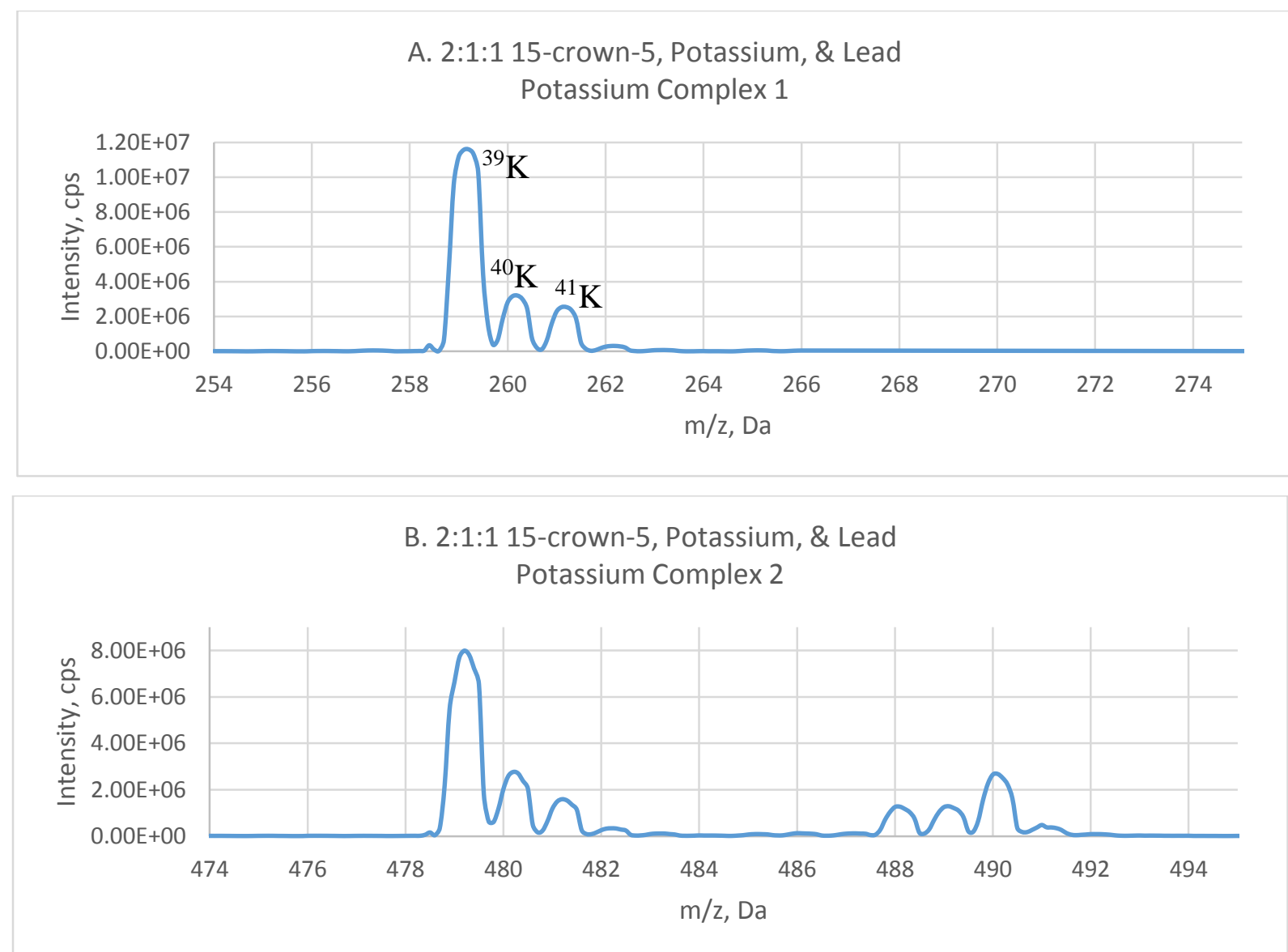

Figure 4.2 A \& B Diluted 2:1:1 potassium and lead solution used for potassium complex isotopic signature confirmation. Note - Spectrum range is from 50-1000 Da and the displayed spectrum is zoomed in to the region of interest. 
The identification of complexes and the cations included were confirmed by the identification of the correlating metal isotope in the positive ion MS/MS spectrum of each isotopic peak. The identification of the parent ions and the ions used for confirmation can be found in Table 4.3. Both single (L) and double $\left(\mathrm{L}_{2}\right)$ crown ether complexes were identified for potassium, barium, and lead while only monomer complexes were identified for copper. This can be attributed to the small size of the copper cation in which association with another crown ether molecule is not feasible due to encapsulation of the cation in the crown ether cavity. Additionally, copper is the only target metal to form a non-nitrated L-M complex. Copper has two common oxidation states, +1 and +2 . The formation of an L-M and L-M-NO 3 complex suggest that the solution is a mixture of copper in its 2 most common oxidation states. The greater intensity of the $\mathrm{L}-\mathrm{M}-\mathrm{NO}_{3}$ complex relative to the L-M complex supports that the +2 oxidation state is the most commonly observed oxidation state for copper under these conditions.

The nitrate arises from the nitric acid matrices of the ICP-MS standard metal solutions. A metal nitrate ion was observed in the MS/MS spectra for lead and barium complexes. The fragment correlated with the correct metal isotope of the parent ion selected. Due to the lack of presence of bare barium isotope fragments, the $\mathrm{M}-\mathrm{NO}_{3}$ fragment was used to confirm the identification of barium complexes. 
Table 4.3 Ions observed for the 1:1 crown ether metal mixtures and the fragment ion used to confirm the identification of the complex in which "L" represents 15-crown-5.

\begin{tabular}{|c|c|c|c|}
\hline Metal & Ions $(m / z)$ observed $(\mathrm{Q} 1)$ & Identification & Confirmatory Ions (Q3) \\
\hline \multirow[t]{10}{*}{ Barium } & 420 & {$\left[\mathrm{~L}+{ }^{138} \mathrm{Ba}+\mathrm{NO}_{3}\right]^{+}$} & $200\left[{ }^{138} \mathrm{Ba}+\mathrm{NO}_{3}\right]^{+}$ \\
\hline & 419 & {$\left[\mathrm{~L}+{ }^{137} \mathrm{Ba}+\mathrm{NO}_{3}\right]^{+}$} & $199\left[{ }^{137} \mathrm{Ba}+\mathrm{NO}_{3}\right]^{+}$ \\
\hline & 418 & {$\left[\mathrm{~L}+{ }^{136} \mathrm{Ba}+\mathrm{NO}_{3}\right]^{+}$} & $198\left[{ }^{136} \mathrm{Ba}+\mathrm{NO}_{3}\right]^{+}$ \\
\hline & 417 & {$\left[\mathrm{~L}+{ }^{135} \mathrm{Ba}+\mathrm{NO}_{3}\right]^{+}$} & $197\left[{ }^{135} \mathrm{Ba}+\mathrm{NO}_{3}\right]^{+}$ \\
\hline & 416 & {$\left[\mathrm{~L}+{ }^{134} \mathrm{Ba}+\mathrm{NO}_{3}\right]^{+}$} & $196\left[{ }^{134} \mathrm{Ba}+\mathrm{NO}_{3}\right]^{+}$ \\
\hline & 640 & {$\left[\mathrm{~L}_{2}+{ }^{138} \mathrm{Ba}+\mathrm{NO}_{3}\right]^{+}$} & $\begin{array}{c}200\left[{ }^{138} \mathrm{Ba}+\mathrm{NO}_{3}\right]^{+} \\
420\left[\mathrm{~L}+{ }^{138} \mathrm{Ba}+\mathrm{NO}_{3}\right]^{+}\end{array}$ \\
\hline & 639 & {$\left[\mathrm{~L}_{2}+{ }^{137} \mathrm{Ba}+\mathrm{NO}_{3}\right]^{+}$} & $\begin{array}{c}199\left[{ }^{137} \mathrm{Ba}+\mathrm{NO}_{3}\right]^{+} \\
419\left[\mathrm{~L}+{ }^{137} \mathrm{Ba}+\mathrm{NO}_{3}\right]^{+}\end{array}$ \\
\hline & 638 & {$\left[\mathrm{~L}_{2}+{ }^{136} \mathrm{Ba}+\mathrm{NO}_{3}\right]^{+}$} & $\begin{array}{c}198\left[{ }^{136} \mathrm{Ba}+\mathrm{NO}_{3}\right]^{+} \\
418\left[\mathrm{~L}_{2}+{ }^{136} \mathrm{Ba}+\mathrm{NO}_{3}\right]^{+}\end{array}$ \\
\hline & 637 & {$\left[\mathrm{~L}_{2}+{ }^{135} \mathrm{Ba}+\mathrm{NO}_{3}\right]^{+}$} & $\begin{array}{c}197\left[{ }^{135} \mathrm{Ba}+\mathrm{NO}_{3}\right]^{+} \\
417\left[\mathrm{~L}_{2}+{ }^{135} \mathrm{Ba}+\mathrm{NO}_{3}\right]^{+}\end{array}$ \\
\hline & 636 & {$\left[\mathrm{~L}_{2}+{ }^{134} \mathrm{Ba}+\mathrm{NO}_{3}\right]^{+}$} & $\begin{array}{c}196\left[{ }^{134} \mathrm{Ba}+\mathrm{NO}_{3}\right]^{+} \\
416\left[\mathrm{~L}_{2}+{ }^{134} \mathrm{Ba}+\mathrm{NO}_{3}\right]^{+}\end{array}$ \\
\hline \multirow[t]{4}{*}{ Copper } & 283 & {$\left[\mathrm{~L}+{ }^{63} \mathrm{Cu}\right]^{+}$} & $63\left[{ }^{63} \mathrm{Cu}\right]^{+}$ \\
\hline & 285 & {$\left[\mathrm{~L}+{ }^{65} \mathrm{Cu}\right]^{+}$} & $65\left[{ }^{65} \mathrm{Cu}\right]^{+}$ \\
\hline & 345 & {$\left[\mathrm{~L}+{ }^{63} \mathrm{Cu}+\mathrm{NO}_{3}\right]^{+}$} & $\begin{array}{c}63\left[{ }^{63} \mathrm{Cu}\right]^{+} \\
283\left[\mathrm{~L}+{ }^{63} \mathrm{Cu}\right]^{+}\end{array}$ \\
\hline & 347 & {$\left[\mathrm{~L}+{ }^{65} \mathrm{Cu}+\mathrm{NO}_{3}\right]^{+}$} & $\begin{array}{c}65\left[{ }^{65} \mathrm{Cu}\right]^{+} \\
285\left[\mathrm{~L}+{ }^{65} \mathrm{Cu}\right]^{+}\end{array}$ \\
\hline \multirow[t]{4}{*}{ Potassium } & 259 & {$\left[\mathrm{~L}+{ }^{39} \mathrm{~K}\right]^{+}$} & $39\left[{ }^{39} \mathrm{~K}\right]^{+}$ \\
\hline & 261 & {$\left[\mathrm{~L}+{ }^{41} \mathrm{~K}\right]^{+}$} & $41\left[{ }^{41} \mathrm{~K}\right]^{+}$ \\
\hline & 479 & {$\left[\mathrm{~L}_{2}+{ }^{39} \mathrm{~K}\right]^{+}$} & $\begin{array}{c}39\left[{ }^{39} \mathrm{~K}\right]^{+} \\
259\left[\mathrm{~L}+{ }^{39} \mathrm{~K}\right]^{+}\end{array}$ \\
\hline & 481 & {$\left[\mathrm{~L}_{2}+{ }^{41} \mathrm{~K}\right]^{+}$} & $\begin{array}{c}41\left[{ }^{41} \mathrm{~K}\right]^{+} \\
261\left[\mathrm{~L}+{ }^{41} \mathrm{~K}\right]^{+}\end{array}$ \\
\hline \multirow[t]{2}{*}{ Lead } & 490 & {$\left[\mathrm{~L}+{ }^{208} \mathrm{~Pb}+\mathrm{NO}_{3}\right]^{+}$} & $\begin{array}{c}208\left[{ }^{208} \mathrm{~Pb}\right]^{+} \\
270\left[{ }^{208} \mathrm{~Pb}+\mathrm{NO}_{3}\right]^{+}\end{array}$ \\
\hline & 489 & {$\left[\mathrm{~L}+{ }^{207} \mathrm{~Pb}+\mathrm{NO}_{3}\right]^{+}$} & $\begin{array}{c}207\left[\left[^{207} \mathrm{~Pb}\right]^{+}\right. \\
269\left[{ }^{207} \mathrm{~Pb}+\mathrm{NO}_{3}\right]^{+}\end{array}$ \\
\hline
\end{tabular}


Table 4.3 Continued

\begin{tabular}{|c|c|c|c|}
\hline Metal & Ions $(m / z)$ observed $(\mathrm{Q} 1)$ & Identification & Confirmatory Ions (Q3) \\
\hline \multirow[t]{6}{*}{$\begin{array}{r}\text { Lead } \\
\text { continued }\end{array}$} & 488 & {$\left[\mathrm{~L}+{ }^{206} \mathrm{~Pb}+\mathrm{NO}_{3}\right]^{+}$} & $\begin{array}{c}206\left[{ }^{206} \mathrm{~Pb}\right]^{+} \\
268\left[{ }^{206} \mathrm{~Pb}+\mathrm{NO}_{3}\right]^{+}\end{array}$ \\
\hline & 486 & {$\left[\mathrm{~L}+{ }^{204} \mathrm{~Pb}+\mathrm{NO}_{3}\right]^{+}$} & $\begin{array}{c}204\left[{ }^{204} \mathrm{~Pb}\right]^{+} \\
266\left[{ }^{204} \mathrm{~Pb}+\mathrm{NO}_{3}\right]^{+}\end{array}$ \\
\hline & 710 & {$\left[\mathrm{~L}_{2}+{ }^{208} \mathrm{~Pb}+\mathrm{NO}_{3}\right]^{+}$} & $\begin{array}{c}208\left[{ }^{208} \mathrm{~Pb}\right]^{+} \\
270\left[{ }^{208} \mathrm{~Pb}+\mathrm{NO}_{3}\right]^{+} \\
490\left[\mathrm{~L}+{ }^{208} \mathrm{~Pb}+\mathrm{NO}_{3}\right]^{+}\end{array}$ \\
\hline & 709 & {$\left[\mathrm{~L}_{2}+{ }^{207} \mathrm{~Pb}+\mathrm{NO}_{3}\right]^{+}$} & $\begin{array}{c}207\left[^{207} \mathrm{~Pb}\right]^{+} \\
269\left[{ }^{207} \mathrm{~Pb}+\mathrm{NO}_{3}\right]^{+} \\
489\left[\mathrm{~L}+{ }^{207} \mathrm{~Pb}+\mathrm{NO}_{3}\right]^{+}\end{array}$ \\
\hline & 708 & {$\left[\mathrm{~L}_{2}+{ }^{206} \mathrm{~Pb}+\mathrm{NO}_{3}\right]^{+}$} & $\begin{array}{c}206\left[^{206} \mathrm{~Pb}\right]^{+} \\
268\left[{ }^{206} \mathrm{~Pb}+\mathrm{NO}_{3}\right]^{+} \\
488\left[\mathrm{~L}+{ }^{206} \mathrm{~Pb}+\mathrm{NO}_{3}\right]^{+}\end{array}$ \\
\hline & 706 & {$\left[\mathrm{~L}_{2}+{ }^{204} \mathrm{~Pb}+\mathrm{NO}_{3}\right]^{+}$} & $486\left[\mathrm{~L}+{ }^{204} \mathrm{~Pb}+\mathrm{NO}_{3}\right]^{+}$ \\
\hline
\end{tabular}

The spectra and the corresponding isotopic signatures were compared to those generated from the Scientific Instrument Services, Inc. Isotope Distribution Calculator and Mass Spec Plotter for correlation. ${ }^{31}$ These molecular formula used to generate each spectrum is located below each reference spectrum in Appendix C. Peaks with an intensity less than 1\% in the generated spectra were typically not resolved in the experimental Q1 spectra. Therefore confirmatory product ion scans were only reported for peaks corresponding with metal isotopes of a natural abundance of $>1 \%$.

Initial screening studies failed to detect antimony 15 -crown-5 complexes in positive or negative ion mode, agreeing with other preliminary studies conducted in our research group. Issues detecting antimony have been previously reported in GSR analysis ${ }^{32-37}$ and unpublished work in our laboratory suggests that solubility may be a key factor in authentic GSR samples. Formation of a white precipitate in the $3.0 \times 10^{-3} \mathrm{M}$ antimony working solution, while not specifically tested, supports this. Additional possibilities for the lack of antimony complex 
identification include the lack of or minimal (below LOD) complex formation, complex dissociation in the spray process, and/or the lack of detection.

Few literature reports regarding complexation of antimony and crown ethers were located. A reference in which antimony has been complexed with 18 -crown- 6 for extraction in an acidic solution and other ligands was located but references for the complexation with 15-

crown-5 were not. ${ }^{38,39}$ Based on the successful complexation with 18 -crown- 6 and relevant atomic data for antimony (size and electronegativity), complexation would be expected. The reason for not detecting antimony complexes is unknown. With respect to the screening performed, it is possible that the complex was overlooked due to association with solvent molecule, like lead, barium, and copper are with nitrate. Similarly to barium, it is likely that the bare metal isotope is not present in the MS/MS spectra and unknown association prevents its identification.

\subsubsection{Reduction of Metal Cations}

An interesting and unanticipated finding in the present studies was the reduction of metal cations from +2 oxidation state to $a+1$ state. This was first noted with lead in which lead crown ether complexes depicted in Q1 spectra included lead as the +2 cation and as the +1 cation in the Q3 (MS/MS) spectra. Figure 4.3 depicts the process the samples undergo from solution to MS/MS spectra. Complexes form in solution and data and literature support that the complexes remain intact during the electrospray process. While metal reduction in the spray process has been observed and reported in literature, it appears that something else is occurring here. ${ }^{28}$ The observed complexes in Q1 and product ions in Q3 suggest that the reduction is occurring in the collision cell (q2) rather than in the electrospray process. Table 4.4 contains the observed 
complexes at the Q1 and Q3 stages of each metal, for the most abundant isotope. Product ion spectra (Q3) are located in Appendix C (FigureC4.3-4.6).

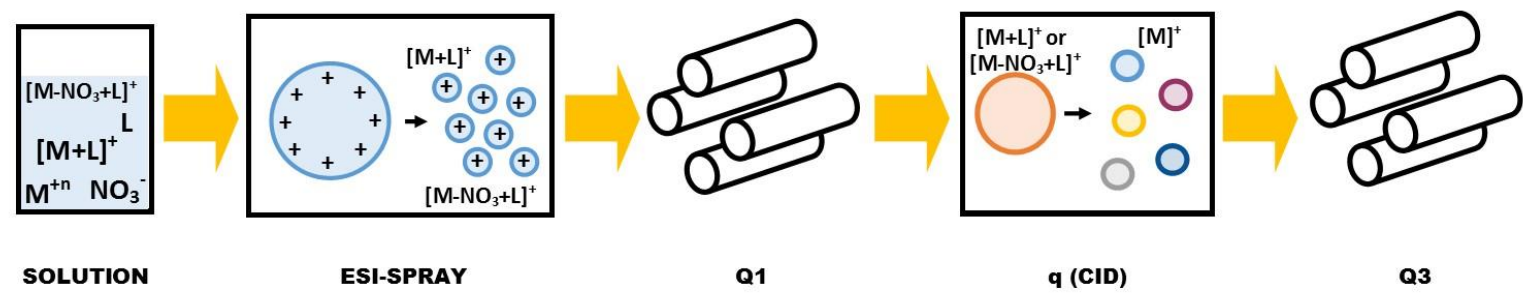

Figure 4.3 Sample analysis process.

Table 4.4 Observed complexes for each target metal in Q1 and Q3 spectra. The $m / z$ values listed are those associated with the most abundant isotope.

\begin{tabular}{|c|c|c|}
\hline Metal & Q1 $(m / z)$ & Q3 $(m / z)$ \\
\hline \multirow{3}{*}{ Barium } & {$\left[\mathrm{Ba}+\mathrm{NO}_{3}\right]^{+}(200)$} & {$[\mathrm{Ba}]^{+}(138)$} \\
\hline & {$\left[15-5+\mathrm{Ba}+\mathrm{NO}_{3}\right]^{+}(420)$} & {$\left[\mathrm{Ba}+\mathrm{NO}_{3}\right]^{+}(200)$} \\
\hline & {$\left[15-5_{2}+\mathrm{Ba}+\mathrm{NO}_{3}\right]^{+}(640)$} & {$\left[\mathrm{Ba}-\mathrm{NO}_{3}\right]^{+}(200) \quad\left[15-5+\mathrm{Ba}+\mathrm{NO}_{3}\right]^{+}(420)$} \\
\hline \multirow{2}{*}{ Copper } & {$[15-5+\mathrm{Cu}]^{+}(283)$} & {$[\mathrm{Cu}]^{+}(63)$} \\
\hline & {$\left[15-5+\mathrm{Cu}+\mathrm{NO}_{3}\right]^{+}(345)$} & {$[\mathrm{Cu}]^{+}(63) \quad[15-5+\mathrm{Cu}]^{+}(283)$} \\
\hline \multirow{2}{*}{ Potassium } & {$[15-5+\mathrm{K}]^{+}(259)$} & {$[\mathrm{K}]^{+}(39)$} \\
\hline & {$\left[15-5_{2}+\mathrm{K}\right]^{+}(479)$} & {$[15-5+\mathrm{K}]^{+}(259)$} \\
\hline \multirow{2}{*}{ Lead } & {$\left[15-5+\mathrm{Pb}+\mathrm{NO}_{3}\right]^{+}(490)$} & {$\left[\mathrm{Pb}+\mathrm{NO}_{3}\right]^{+}(270)$} \\
\hline & {$\left[15-5_{2}+\mathrm{Pb}+\mathrm{NO}_{3}\right]^{+}(710)$} & $\begin{array}{c}{[\mathrm{Pb}]^{+}(208) \quad\left[\mathrm{Pb}+\mathrm{NO}_{3}\right]^{+}(270)} \\
{\left[15-5+\mathrm{Pb}+\mathrm{NO}_{3}\right]^{+}(490)}\end{array}$ \\
\hline
\end{tabular}


Using lead as an example, a hypothesized reaction taking place in the collision cell is:

$$
\begin{gathered}
\mathrm{Pb}^{2+}+\mathrm{e}^{-} \rightarrow \mathrm{Pb}^{+}(\mathrm{EA}=-15.032 \mathrm{eV})^{40} \\
\mathrm{NO}_{3}{ }^{-} \rightarrow \mathrm{NO}_{3}+\mathrm{e}^{-}(\mathrm{EA}=3.9 \mathrm{eV})^{40} \\
\Delta \mathrm{H}=3.9 \mathrm{eV}-15.03 \mathrm{eV}=-11.1 \mathrm{eV}
\end{gathered}
$$

The reaction is energetically feasible and the nitrate is assumed to be a reducing agent for the nitrated ligand complexes. Fragmentation of the precursor ion $\mathrm{BaNO}_{3}$ (peak at $m / z$ 200) and the observed reduction to $\mathrm{Ba}^{+}$(peak at $m / z$ 138) supports this prediction (Figure 4.4). With the precursor ion $\mathrm{BaNO}_{3}$, the crown ether moiety is not present in the collision cell to act as the reducing agent yet barium is still reduced from a +2 oxidation state to a +1 oxidation state indicating that the reducing agent is $\mathrm{NO}_{3}$.

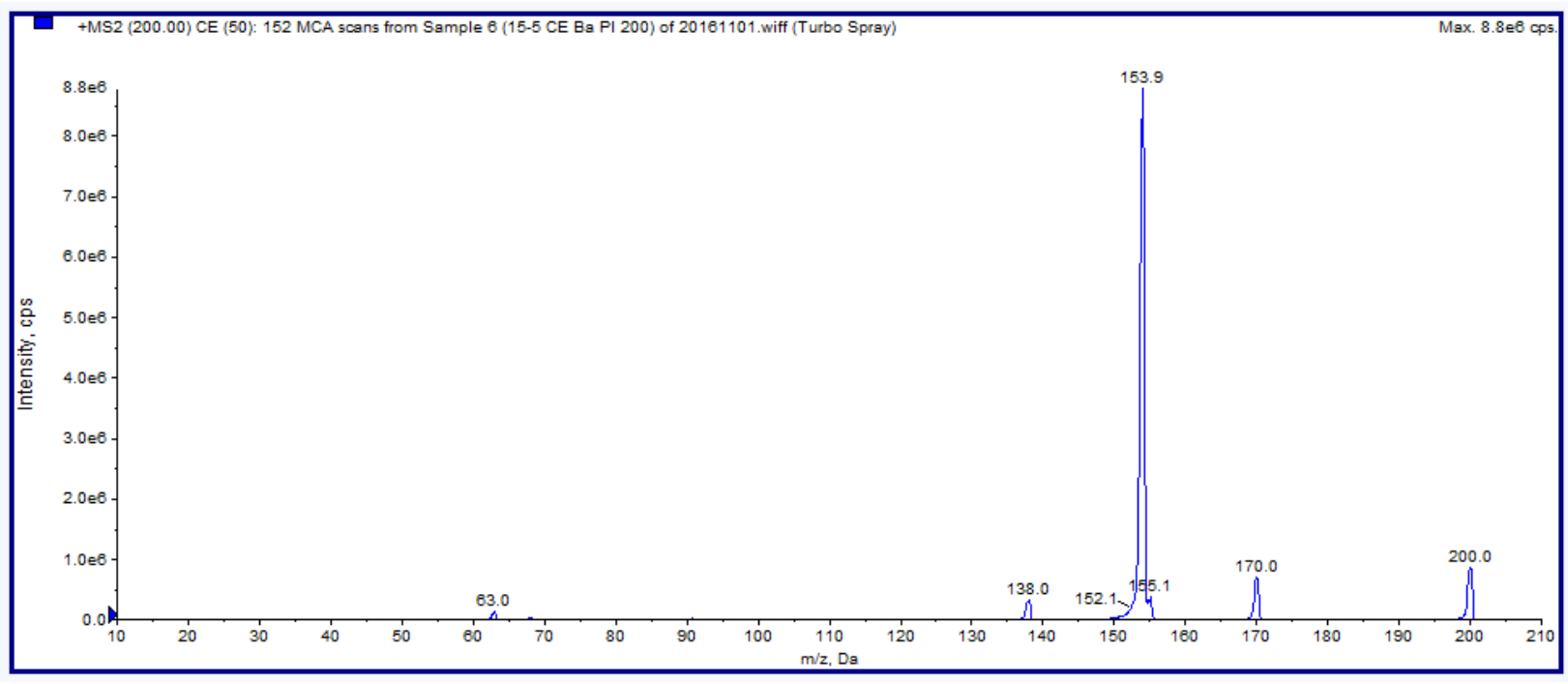

Figure 4.4 MS/MS spectrum of precursor ion peak at $m / z 200\left(\mathrm{BaNO}_{3}\right)$ in which the ${ }^{138} \mathrm{Ba}$ isotope is observed. 
Interestingly, all nitrated cations $(\mathrm{Ba}, \mathrm{Cu}$, and $\mathrm{Pb})$ were being reduced to the +1 state in the CID. This is evident due the change in oxidation state from Q1 to Q3 observed in the corresponding spectra. The nitrated metal complexes are observed in the Q1 spectra at an $\mathrm{m} / \mathrm{z}$ that corresponds to $\mathrm{z}=1$. An example is seen in the Q1 (MS) spectrum in Figure 4.5A of the $\left[15-5+\mathrm{Pb}+\mathrm{NO}_{3}\right]^{+}$complex. The peak at $m / 2,490$ indicates that the oxidation state of lead is a +2. When the nitrated metal complex ions are selected as parent ions and undergo CID, fragments associated with the metal isotope are also at an $\mathrm{m} / \mathrm{z}$ that corresponds to a $\mathrm{z}=1$, Figure 4.5B. Therefore, this observation suggests that reduction is a result of reactions taking place in the collision cell rather than the electrospray process.

The association with $\mathrm{NO}_{3}$ and the reduction as a result of that association indicates that care must be taken during method development and spectra interpretation if this method is to be applied to authentic GSR samples. Association with solvent molecules other than $\mathrm{NO}_{3}$ or $\mathrm{LC}$ mobile phase molecules could occur thus changing the parent and product ions $m / z$ values of the complexes or potentially inhibiting complexation. Therefore compound identification must be performed if solvent changes occur in the extraction protocol or analysis process. 

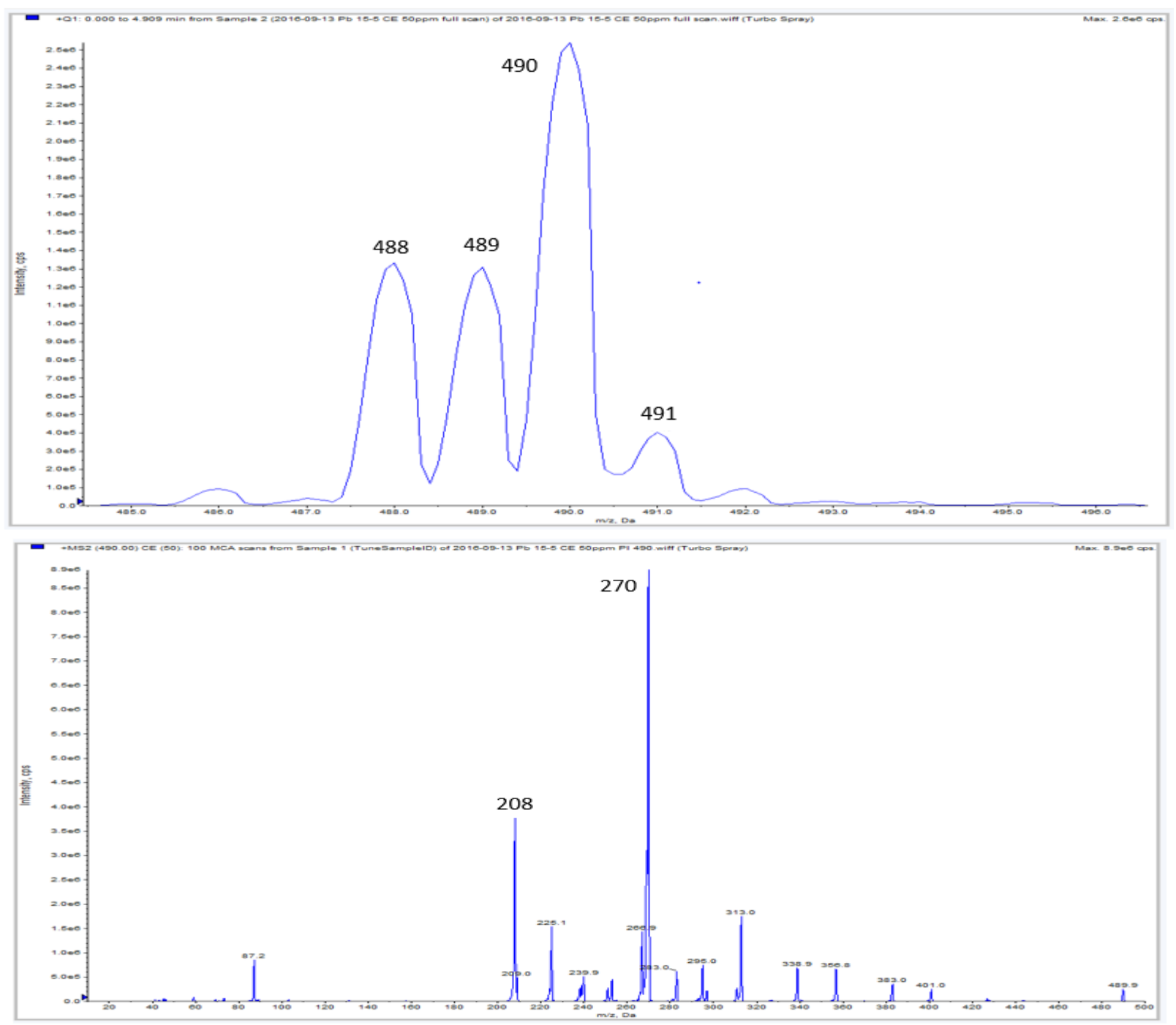

Figure 4.5 A. Q1(MS) spectrum of the $\left[15-5+\mathrm{Pb}+\mathrm{NO}_{3}\right]^{+}$complex in which the oxidation state of $\mathrm{Pb}$ is a +2 . B. Q3(MS/MS) spectrum of the parent ion $m / z 490\left(\left[15-5+\mathrm{Pb}+\mathrm{NO}_{3}\right]^{+}\right)$complex in which the oxidation state of $\mathrm{Pb}$ is +1 .

\subsubsection{Complex Abundance}

After confirmation of the composition of the metal complexes, compound optimization was performed for each complex. A Q1 multiple ion scan was performed for each 1:1 crown ether metal solution with the optimized conditions. Ion intensity averages $(n=3)$ of the single ligand complexes (L) for barium and lead were typically an order of magnitude higher than that 
of the sandwich complex $\left(\mathrm{L}_{2}\right)$. Although the intensity difference for the potassium complexes was relatively low, the average intensity for the $\mathrm{L}$ complex is higher than that of the $\mathrm{L}_{2}$ complex.

In the case of copper, the average intensity for the non-nitrated complex is higher than that of the nitrated complex. Complex distributions between the two forms of ligand complexes were calculated (Table 4.5.). Two assumptions were made: (1) the desolvation of the two forms of ligand complexes is similar ${ }^{20}$ and (2) the combined abundance is equal to $100 \%$. Based on these assumptions, the results indicate that the barium and lead L:M:NO 3 complexes, or L:M complex in the case of potassium, were more favorable than the $\mathrm{L}_{2}: \mathrm{M}: \mathrm{NO}_{3}$ or $\mathrm{L}_{2}: \mathrm{M}$ complex. For copper the L:M appears more favorable than the L:M:NO 3 complex.

Table 4.5 Calculated relative percent abundance for each metal complex. Values were calculated using average intensity $(n=3)$ of the most abundant isotope with the exception of potassium (second most abundant isotope).

\begin{tabular}{rcc}
\hline Metal & Complex & Percent Abundance \\
\hline Barium & {$\left[15-5+\mathrm{Ba}+\mathrm{NO}_{3}\right]^{+}(420)$} & $86 \%$ \\
\hline Copper & {$\left[15-52+\mathrm{Ba}+\mathrm{NO}_{3}\right]^{+}(640)$} & $14 \%$ \\
\hline & {$\left[15-5+\mathrm{Cu}+\mathrm{NO}_{3}\right]^{+}(345)$} & $65 \%$ \\
\hline Potassium & {$\left[15-5+\mathrm{K}^{+}(261)\right.$} & $35 \%$ \\
\hline & {$\left[15-5_{2}+\mathrm{K}^{+}(481)\right.$} & $65 \%$ \\
\hline Lead & {$\left[15-5+\mathrm{Pb}+\mathrm{NO}_{3}\right]^{+}(490)$} & $35 \%$ \\
\hline
\end{tabular}




\subsubsection{Binding Selectivities}

The ability to simultaneously analyze multiple complexes with ESI-MS allows competitive binding studies to be performed and information on relative competitiveness to be studied. ${ }^{41}$ ESI-MS has been used by several groups to successfully determine binding selectivity's for crown ethers and related macrocycles and metals. ${ }^{16,18,20,22,42}$ The studies presented here were aimed at evaluating the relative competitiveness of GSR metals in an effort to understand the complexation relative to a complex GSR system.

It is known that evaluating mixtures through spectral ratios does not take into account ionization, desolvation, and transmission efficiencies. ${ }^{18}$ During complexation a metal ion is encapsulated by the host molecule and because of this ionization, desolvation, and transmission efficiencies of the complex is largely a function of the host molecule rather than the guest. ${ }^{16,20}$ Therefore, the ionization, desolvation, and transmission efficiencies of complexes with the same or similar hosts will be similar. Thus, spectral ion intensities of the complexes are a representation of the solution equilibrium distribution and spectral ratios can be used to evaluate mixtures containing a single host and multiple guests. ${ }^{20}$

Mixtures of crown ether and two metals (potassium, barium, copper or lead) were combined in a 2:1:1 ratio and analyzed. Potassium has a high affinity for crown ether and has been extensively studied and as such it was selected as a reference to provide insight on the competition occurring and the relative competitiveness of the metals. Mass spectral intensities of the complexes were used to calculate experimental values and are expressed as the percentage of total metal complexes (\%T $[\mathrm{M}])$. These values were calculated with 4 different methods by summing various isotopic mass spectral intensities from the two types of metal complexes. This was done to determine the most accurate representation of the mixture. Method 1 and 2 utilized 
the most abundant isotope in which method 1 included the mass spectral intensities of the most abundant isotope of the favored complex (Table 4.6) and method 2 included the most abundant isotope in both complexes. For example, for method 2 the total percent of the copper complex in the 2:1:1 15-crown-5:Cu:Ba mixture was calculated as follows:

$$
\% T[C u]=\frac{\left[15: 5+63_{\mathrm{Cu}}\right]+\left[15: 5+63_{\mathrm{Cu}}+\mathrm{NO}_{3}\right]}{\left[15: 5+138_{\mathrm{Ba}}+\mathrm{NO}_{3}\right]+\left[15: 5_{2}+138_{\mathrm{Ba}}+\mathrm{NO}_{3}\right]+\left[15: 5+63_{\mathrm{Cu}}\right]+\left[15: 5+63_{\mathrm{Cu}}+\mathrm{NO}_{3}\right]} \times 100
$$

\section{Equation 4.1}

Conversely, method 3 and 4 utilized all metal isotopic peaks; method 3: all metal isotopic peaks in the favored complex and method 4: all metal isotopic peaks in both complexes. Table $\mathbf{4 . 5}$ provides an example, using the 2:1:1 15-crown-5:Cu:Ba mixture, of the peaks utilized for each methods calculations.

Table 4.6 The peaks used to calculate the $\% \mathrm{~T}[\mathrm{M}]$ for each method utilizing the 2:1:1 mixture of 15-crown-5, copper and barium as an example. The peaks used for the other mixtures are located in Appendix C.

\begin{tabular}{lc}
\hline Method & Complex $(\boldsymbol{m} / \mathbf{z})$ \\
\hline Method 1 & {$\left[15-5+{ }^{63} \mathrm{Cu}\right]^{+}(283)$} \\
\hline Method 2 & {$\left[15-5+{ }^{138} \mathrm{Ba}+\mathrm{NO}_{3}\right]^{+}(420)$} \\
\hline Method 3 & {$\left[15-5+{ }^{63} \mathrm{Cu}\right]^{+}(283),\left[15-5+{ }^{63} \mathrm{Cu}+\mathrm{NO}_{3}\right]^{+}(345)$} \\
\hline & {$\left[15-5+{ }^{138} \mathrm{Ba}+\mathrm{NO}\right]^{+}(420),\left[15-52+{ }^{138} \mathrm{Ba}+\mathrm{NO}_{3}\right]^{+}(640)$} \\
\hline & {$\left[15-5+{ }^{63} \mathrm{Cu}\right]^{+}(283),\left[15-5+{ }^{65} \mathrm{Cu}\right]^{+}(285)$} \\
\hline $\left.\mathrm{NO}_{3}\right]^{+}(416),\left[15-5+{ }^{137} \mathrm{Ba}+\mathrm{NO}_{3}\right]^{+}(419),\left[15-5+{ }^{138} \mathrm{Ba}+\mathrm{NO}_{3}\right]^{+}(420)$
\end{tabular}




\begin{tabular}{c}
\hline Table 4.5 Continued \\
\hline$\left[15-5+{ }^{63} \mathrm{Cu}\right]^{+}(283),\left[15-5+{ }^{65} \mathrm{Cu}\right]^{+}(285)$, \\
{$\left[15-5+{ }^{63} \mathrm{Cu}+\mathrm{NO}_{3}\right]^{+}(345),\left[15-5+{ }^{65} \mathrm{Cu}+\mathrm{NO}_{3}\right]^{+}(347)$} \\
\hline Method 4 \\
{$\left[15-5+{ }^{134} \mathrm{Ba}+\mathrm{NO}_{3}\right]^{+}(416),\left[15-5+{ }^{135} \mathrm{Ba}+\mathrm{NO}_{3}\right]^{+}(417),\left[15-5+{ }^{136} \mathrm{Ba}+\mathrm{NO}_{3}\right]^{+}(418)$,} \\
{$\left[15-5_{2}+{ }^{137} \mathrm{Ba}+\mathrm{NO}_{3}\right]^{+}(419),\left[15-5+\mathrm{NO}_{3}\right]^{+}(637),\left[15-5_{2}+{ }^{136} \mathrm{Ba}+\mathrm{NO}_{3}\right]^{+}(420)$,} \\
{$\left[15-5_{2}+{ }^{137} \mathrm{Ba}+\mathrm{NO}_{3}\right]^{+}(638)$,}
\end{tabular}

Two sample t-tests were performed with corresponding metals between methods 1 and 2, 2 and 3 , and 3 and 4 to determine if the $\% \mathrm{~T}[\mathrm{M}]$ of each method were significantly different. Assuming unequal variance, the resulting p-values were less than $5 \%$ with the exception of the two-tail results of the comparison of methods 2 and 3 for the 2:1:1 mixture containing potassium $(6.7 \%)$ and copper $(6.7 \%)$. This means that there is a less than $5 \%$ chance that the two sets came from the same group indicating that all 4 methods are significantly different from one another, excluding the previously mentioned exception. In addition, due to the previous mentioned peak splitting of the potassium complexes two sets of $\% \mathrm{~T}[\mathrm{~K}]$ values for methods 1 and 2 were calculated using the mass spectral intensities for ${ }^{39} \mathrm{~K}(\mathrm{n}=3)$ and ${ }^{41} \mathrm{~K}(\mathrm{n}=3)$. Two sample $\mathrm{t}$-tests were also performed to determine if the results were significantly different from one another. Again, assuming unequal variance, the p-value in all three mixtures containing potassium (15-5 with $\mathrm{K}: \mathrm{Ba}, \mathrm{K}: \mathrm{Pb}, \mathrm{K}: \mathrm{Cu}$ ) and for both methods (1 and 2) was less than 5\% (0.05). In other words, the $\% \mathrm{~T}[\mathrm{~K}]$ values calculated using the mass spectral intensities for ${ }^{39} \mathrm{~K}$ are significantly different than that calculated using the mass spectral intensities for ${ }^{41} \mathrm{~K}$. 
In addition to calculating experimental \% $\mathrm{T}[\mathrm{M}]$ values, MINEQL+ Chemical Equilibrium Modeling System software (version 5.0, Environmental Research Software, Hallowell, ME) was used, based on its use in literature, to simulate theoretical solution composition and calculate theoretical \% $[\mathrm{M}]{ }^{16}$ The following $\log \mathrm{K}$ values were used in the simulations of the formation of 15-crown-5 metal complexes: $\mathrm{K}=3.63, \mathrm{~Pb}=3.56, \mathrm{Ba}=2.61$, and $\mathrm{Cu}=2.20 .{ }^{43}$ Literature values were collected using methods such as polarography and calorimetry and are based on solutions in methanol with the exception of copper which also contains $0.1 \mathrm{M}$ tetrabutylammonium perchlorate $\left(\mathrm{Bu}_{4} \mathrm{NClO}_{4}\right){ }^{43}$

To progress towards a more realistic GSR sample, a 3:1:1:1 mixture containing 15crown-5, lead, barium and copper was also analyzed in addition to the 2:1:1 mixtures. The average experimental \% $[\mathrm{M}]$ of each metal in the 2:1:1 mixtures and the 3:1:1:1 mixture, calculated using method 4, are reported in Table 4.7 along with the theoretical values. Method 4, which includes all main metal isotopes in both complexes, was selected based on the resulting lowest summation of the differences between the experimental and theoretical values, likely due to the inclusiveness of the calculation. The experimental $\% \mathrm{~T}[\mathrm{M}]$ values have a maximum standard deviation of $0.3 \%(n=3)$ with a $\%$ RSD of $<1 \%$. 
Table 4.7 Calculated experimental and theoretical percent total metal complex (\% $[\mathrm{M}])$ for the metals in the 2:1:1 mixtures of 15-crown-5.

\begin{tabular}{|c|c|c|c|}
\hline Mixture & Metal & $\% \mathrm{~T}[\mathrm{M}]$ Experimental $^{\mathrm{a}}$ & $\% \mathrm{~T}[\mathrm{M}]$ Theoretical ${ }^{\mathrm{b}}$ \\
\hline \multirow{2}{*}{$15: 5+\mathrm{Cu}+\mathrm{Ba}$} & $\mathrm{Cu}$ & $33 \%$ & $33 \%$ \\
\hline & $\mathrm{Ba}$ & $67 \%$ & $67 \%$ \\
\hline \multirow{2}{*}{$15: 5+\mathrm{Cu}+\mathrm{Pb}$} & $\mathrm{Cu}$ & $8 \%$ & $14 \%$ \\
\hline & $\mathrm{Pb}$ & $92 \%$ & $86 \%$ \\
\hline \multirow{2}{*}{$15: 5+\mathrm{K}+\mathrm{Ba}$} & $\mathrm{K}$ & $76 \%$ & $76 \%$ \\
\hline & $\mathrm{Ba}$ & $24 \%$ & $24 \%$ \\
\hline \multirow{2}{*}{$15: 5+\mathrm{K}+\mathrm{Cu}$} & $\mathrm{K}$ & $95 \%$ & $87 \%$ \\
\hline & $\mathrm{Cu}$ & $5 \%$ & $13 \%$ \\
\hline \multirow{2}{*}{$15: 5+\mathrm{K}+\mathrm{Pb}$} & $\mathrm{K}$ & $64 \%$ & $51 \%$ \\
\hline & $\mathrm{Pb}$ & $36 \%$ & $49 \%$ \\
\hline \multirow{3}{*}{$\begin{array}{r}15: 5+\mathrm{Pb}+\mathrm{Ba}+ \\
\mathrm{Cu}\end{array}$} & $\mathrm{Pb}$ & $64 \%$ & $66 \%$ \\
\hline & $\mathrm{Ba}$ & $29 \%$ & $23 \%$ \\
\hline & $\mathrm{Cu}$ & $7 \%$ & $11 \%$ \\
\hline
\end{tabular}

\footnotetext{
${ }^{\mathrm{a}}$ Experimental values were calculated by summing the peak intensities of the designated metal complexes including metal isotopic peaks and dividing by the sum of the peak intensities of both metal complexes and multiplying by 100 .

$\mathrm{b}$ Theoretical concentration values were obtained using MINEQL+ software. The percentage reported was calculated by dividing the concentration of the designated metal complex by the sum of the metal complexes and multiplying by 100 .
}

Although the experimental values differ slightly from the theoretical values, as seen in

Table 4.6, the preferential binding between the metals remains consistent. The differences between theoretical and experimental values may be attributed to the following: (1) selected log $\mathrm{K}$, (2) nitrated metals and sandwich complexes or (3) difference in the exposure of the metal in 
the complex. Multiple log $\mathrm{K}$ values exist in literature for the formation of the complexes of interest and thus error may exist in selecting the $\log \mathrm{K}$ value used for the determination of the theoretical values. In addition, theoretical values were based on the formation of single crown ether complexes with bare metal ions. The nitrated metals and the formation of sandwich complexes were not considered in the theoretical calculation due to the inability to locate $\log \mathrm{K}$ values. Lastly, ionization, desolvation, and transmission of the complex in the ESI process is a function of the interaction of the molecules exposed to the solvent. Although this interaction is believed to be with the organic host, due to the varying sizes and other chemical properties of the metals the metal ions "fit" into the cavity of the host also varies. Therefore, some of the metals are more exposed to the solvent than others thus potentially influencing the ionization, desolvation, and transmission of the complex.

Utilizing the experimentally determined \% $\mathrm{T}[\mathrm{M}]$, which has been directly correlated to binding selectivities of metals to host molecules, ${ }^{16,44}$ the preferential binding relative to the metals of interest was established. With a binding selectivity between $64 \%$ and $94 \%$, as seen in Figure 4.6 which compares the 2:1:1 mixtures and the $3: 1: 1: 1$ mixture, potassium has the greatest binding selectivity relative to the other target metals. Furthermore, copper was determined to have the lowest binding selectivity in which the binding selectivity of copper was never greater than $33 \%$ in the 4 mixtures containing copper. As for barium and lead, the binding selectivities of the 3:1:1:1 mixture indicates that 15-crown-5 prefers lead over barium. Therefore the experimental sequential preferential binding relative to the metals of interest is as follows:
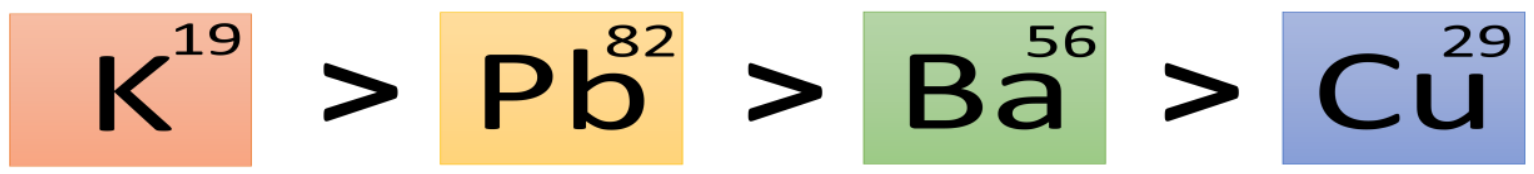


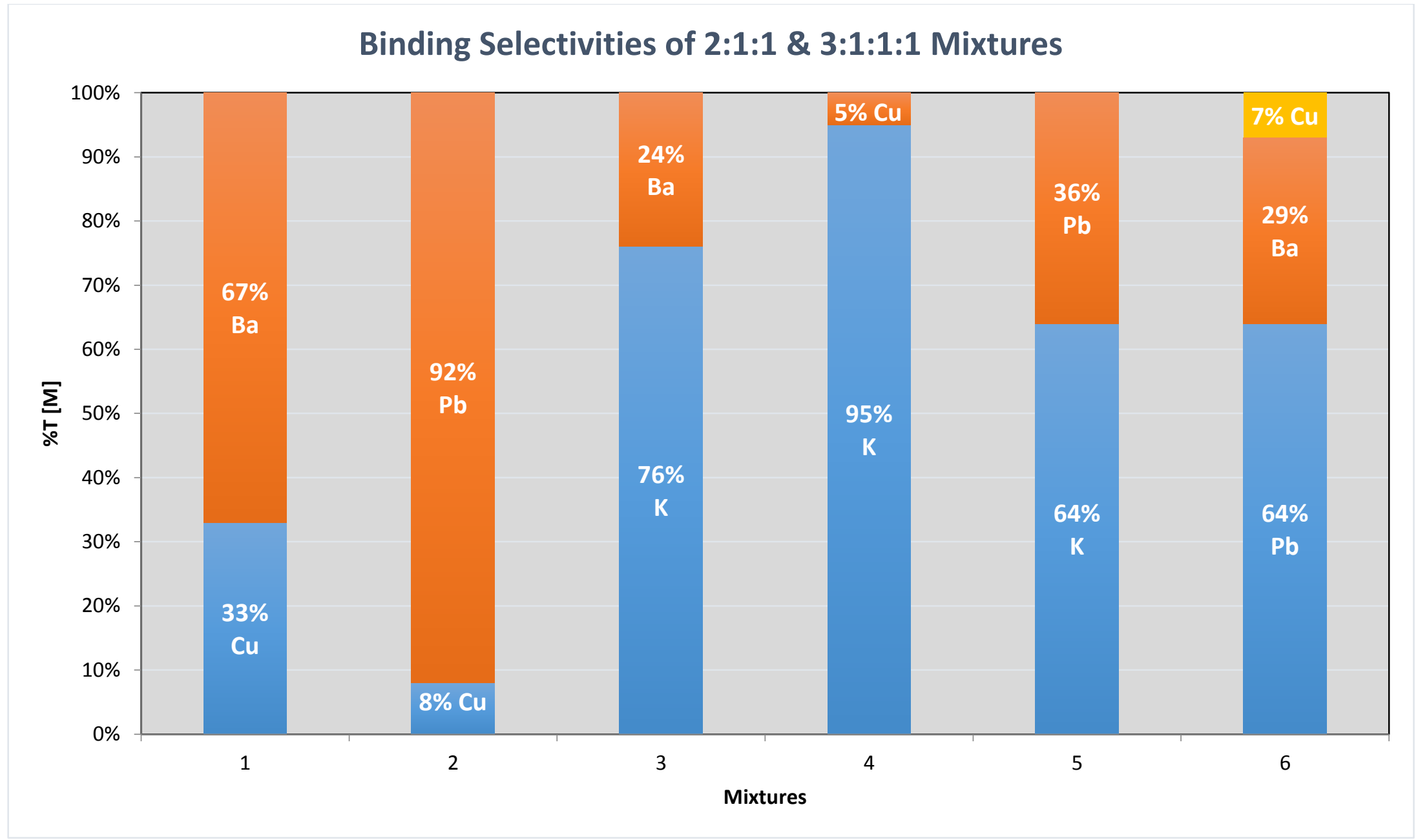

Figure 4.6 Calculated experimental \% $\mathrm{T}[\mathrm{M}]$ of the 2:1:1 and 3:1:1:1 mixtures which were directly translated into binding selectivities of the target metals. 
The determined preferentially binding provides important information if the methods used are to be applied to authentic samples. First, background/contaminant ions, such as potassium, must be removed prior to addition of any host. As a result of their high binding selectivities, potassium and similar ions could cause signal suppression or unwanted competition between metal ions if there was a deficit of complexing agent. In both of these instances important data could be lost or missed. Secondly, it provides a sequence in which metals should be identified. Lead has the highest binding selectivity out of the analyzed GSR metals and therefore an analyst would expect to detect lead prior to the other metals in authentic samples.

\subsubsection{Preliminary Molecular Modeling}

Molecular modeling experiments were performed using ChemDraw 3D Ultra (Ver. 16). Once the structures were created, they were minimized using the MMFF94 (molecular mechanics force field 94) engine with multiprocessor support enabled. This engine, although preferred for larger organic molecules and proteins, afforded easy implementation with metal cations. Convergence was declared when the root mean square gradient reached 0.100. Next, MOPAC engine, a semi-empirical molecular orbital method well suited to conformational determination and ions, was used to further evaluate and minimize the structures.

Preliminary modeling experiments included the formation of the single ligand 15-crown5 complexes with the target metals excluding the nitrate. The resulting minimum energy structures are seen in Figure 4.7. The structures include the measured distance between the target metal ions and the crown ether's oxygen atoms. Directly below the structures the corresponding calculated total energy and enthalpies of formation are recorded along with the ionic radii of the metal ions. The results were integrated into the analysis and interpretation of the experimental results. 
The cavity size of 15 -crown- 5 is determined by the diameter of oxygen $(2.64 \AA)$ subtracted from the O-O distance. ${ }^{26}$ Based on the modeling parameters used the calculated cavity size ranges from 1.86-3.56 $\AA$. While the size of the crown ether cavity and metal ion are playing a role it the formation of the minimum energy structure is apparent that more is contributing to the selectivity and stability of the complex. The complexes with the resulting maximum and minimum metal ionic radii and enthalpies of formation correspond to the complexes with the highest and lowest experimentally determined binding selectivities, the potassium and copper complexes respectively. However, antimony with a slightly larger ionic radius than that of copper did not converge to form a stable complex. These results provided insight into the inability to detect antimony and identify a complex experimentally. Although solubility was believed to be the primary issue, based on the modeling data any ions that were making it into solution were unable to form stable complexes. 


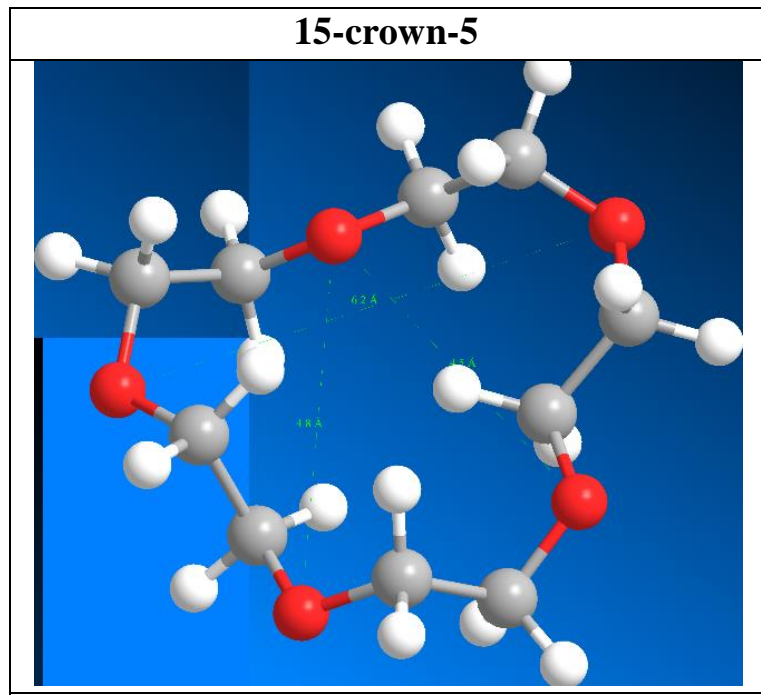

Total Energy: -2973.86 eV $\Delta \mathrm{H}_{\mathrm{f}}:-864.98$ 15-crown-5 Antimony Complex

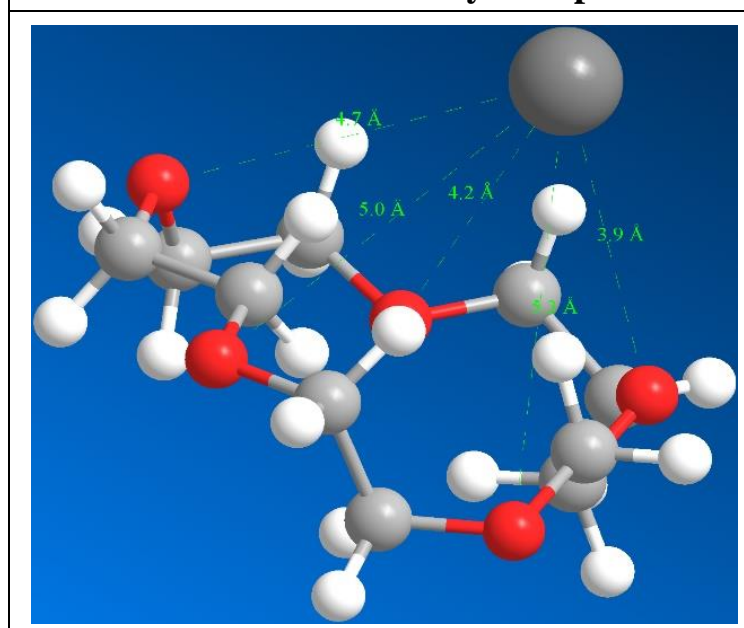

Total Energy: No Convergence $\quad \Delta \mathrm{H}_{\mathrm{f}}: \mathrm{N} / \mathrm{A}$ Ionic Radius of $\mathrm{M}^{2+}: 0.76 \AA$

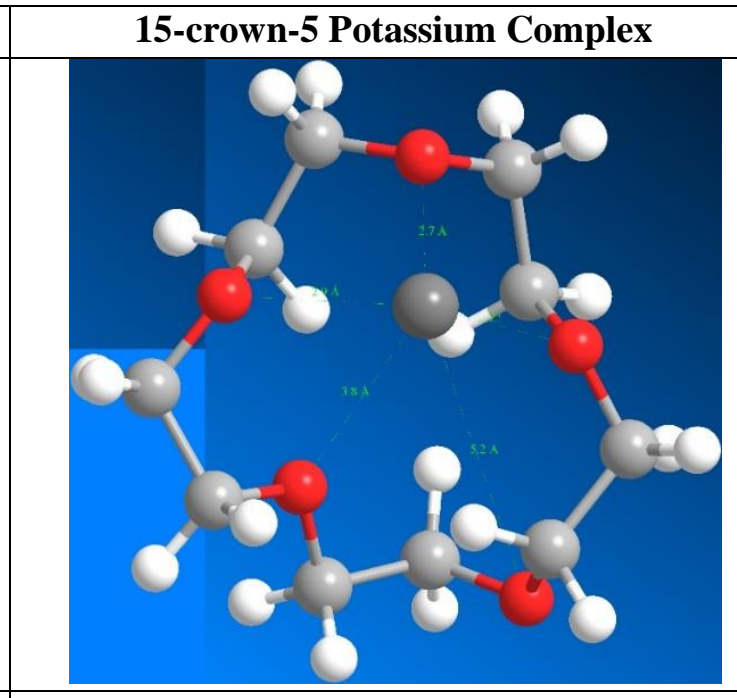

Total Energy: -2975.3 eV $\Delta \mathrm{H}_{\mathrm{f}}:-447.27$ Ionic Radius of $\mathrm{M}^{+}: 1.38 \AA$ 15-crown-5 Barium Complex

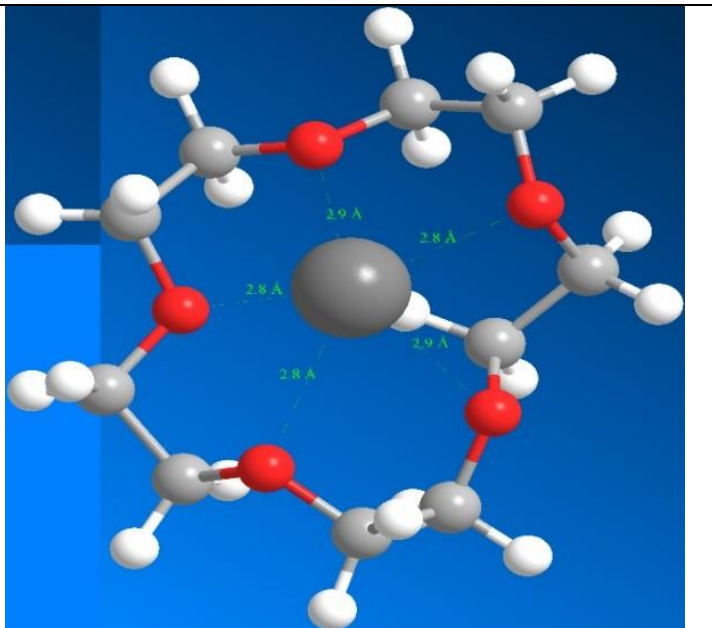

Total Energy: $-2979.10 \mathrm{eV} \quad \Delta \mathrm{H}_{\mathrm{f}}: 283.97$ Ionic Radius of $\mathrm{M}^{2+}: 1.35 \AA$

\section{5-crown-5 Lead Complex}

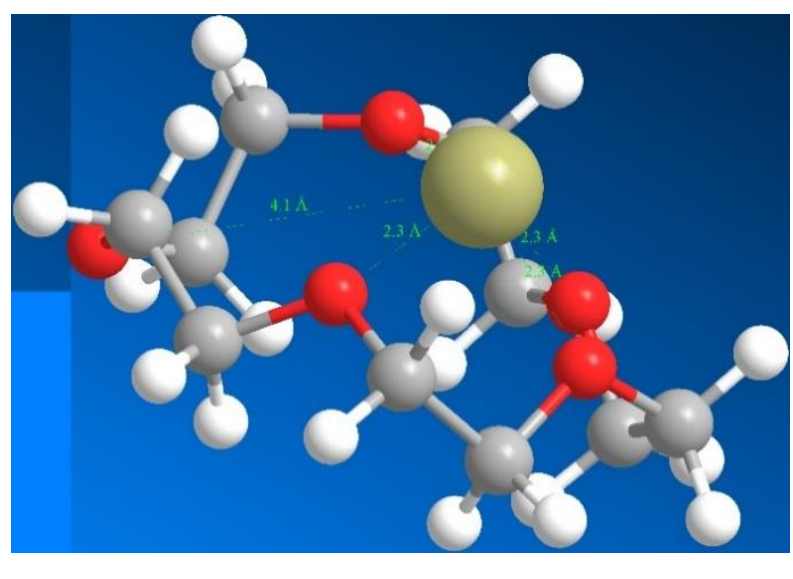

Total Energy: -3050.42 eV $\quad \Delta \mathrm{H}_{\mathrm{f}}: 641.50$ Ionic Radius of $\mathrm{M}^{2+}: 1.19 \AA$

\section{5-crown-5 Copper Complex}

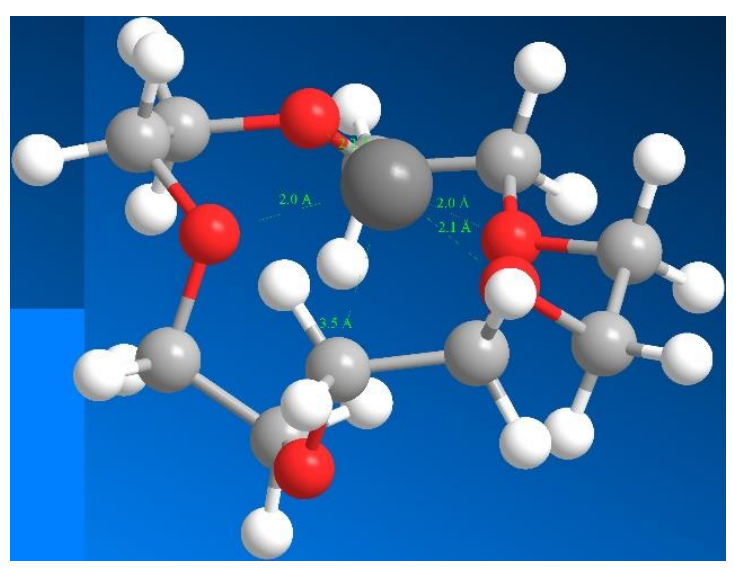

Total Energy: $-3632.66 \mathrm{eV} \quad \Delta \mathrm{H}_{\mathrm{f}}: 858.32$ Ionic Radius of $\mathrm{M}^{2+}: 0.73 \AA$

Figure 4.7 Minimum energy structures of 15 -crown-5 and the metal ions of interest; the yellow sphere and dark grey spheres represent the $\mathrm{M}^{+\mathrm{n}}$ ions. The red (oxygen), white (hydrogen), and light grey (carbon) spheres comprise the crown ether complex. Note that the depicted metal ions are not to scale with their ionic radii. 


\subsection{Conclusions}

While OGSR methods are being researched and developed, little is being done to improve the evidentiary value of GSR. Combining OGSR and GSR analysis is essential for the future of FDR analysis. Complexation affords elemental analysis on an instrument typically reserved for organic compounds, providing a means to analyze OGSR and GSR simultaneously. FDR is a heterogeneous mixture composed of many metals besides the traditionally targeted lead, barium, and antimony. This already complex matrix becomes even more complex when samples are collected from the hands of suspects, adding potassium, sodium, etc. The application of complexation with respect to authentic samples begins with understanding how GSR metals complex individually and what occurs when the complexity of the sample increases towards a more realistic FDR sample. The work presented begins this understanding by demonstrating the complexation of 15-crown-5 with known GSR metals.

Complexation with 15-crown-5 and target GSR samples was successful with the exception of antimony. Single ligand and double ligand sandwich complexes were identified based on isotopic signatures and fragmentation of parent ions down to metal nitrates and bare metals. An unexpected observation made during identification was the reduction of metal cations from +2 oxidation state to $a+1$ state, which upon further observation was deemed a result of CID. Mass spectral intensities of complexes in mixtures were used to calculate binding selectivities. Experimental values were compared to theoretical values and while the trends were consistent, some differences were noted. Furthermore, molecular modeling results were integrated into the analysis and interpretation of the experimental results and more importantly, the general process provided a means to evaluate the potential efficacy of new complexing agents in-silico. 
The studies reported here were performed with standard solutions under semi-controlled conditions while ideal for initial evaluation, authentic FDR samples will provide a much more challenging matrix. While future studies include revisiting the sample collection process in order to maximize the amount of sample collected and the application of the presented work towards authentic FDR hand swabs, additional evaluation of 15-crown-5 or any other hosts must be performed with authentic FDR samples. Extraction procedures of authentic FDR samples involving the reconstitution of the samples in a crown ether solution are currently being researched in our laboratory and prove promising.

\subsection{Funding and Additional Notes}

This work was supported by the National Institute of Justice through the Forensic Center of

Excellence at RTI International, contract \# 2015-DN-BX-K048 (NIJ) and 2011-DN-BX-K564 (RTI/NIJ). 


\subsection{References}

1. O. Dalby, D. Butler and J. W. Birkett, J. Forensic Sci., 2010, 55, 924-943.

2. R. V. Taudte, A. Beavis, L. Blanes, N. Cole, P. Doble and C. Roux, BioMed research international, 2014, 2014, 965403-965403.

3. K. H. Chang, P. T. Jayaprakash, C. H. Yew and A. F. L. Abdullah, Australian Journal of Forensic Sciences, 2013, 45, 3-23.

4. J. W. Moran and S. Bell, Analytical Chemistry, 2014, 86, 6071-6079.

5. T. Jalanti, P. Henchoz, A. Gallusser and M. S. Bonfanti, Sci. Justice, 1999, 39, 48-52.

6. J. Moran and S. Bell, Int. J. Ion Mobil. Spec., 2013, 16, 247-258.

7. J. Arndt, S. Bell, L. Crookshanks, M. Lovejoy, C. Oleska, T. Tulley and D. Wolfe, Forensic Sci.Int., 2012, 222, 137-145.

8. W. Z. Shou and R. F. Browner, Analytical Chemistry, 1999, 71, 3365-3373.

9. J. C. Traeger, Int. J. Mass Spectrom., 2000, 200, 387-401.

10. V. B. Di Marco and G. G. Bombi, Mass Spectrometry Reviews, 2006, 25, 347-379.

11. R. Colton, A. Dagostino and J. C. Traeger, Mass Spectrometry Reviews, 1995, 14, 79106.

12. R. Jirasko and M. Holcapek, Mass Spectrometry Reviews, 2011, 30, 1013-1036.

13. A. T. Blades, P. Jayaweera, M. G. Ikonomou and P. Kebarle, Int. J. Mass Spectrom. Ion Process., 1990, 101, 325-336.

14. A. T. Blades, P. Jayaweera, M. G. Ikonomou and P. Kebarle, Int. J. Mass Spectrom. Ion Process., 1990, 102, 251-267.

15. M. Albrecht, Naturwissenschaften, 2007, 94, 951-966.

16. M. L. Reyzer, J. S. Brodbelt, A. P. Marchand, Z. B. Chen, Z. L. Huang and I. N. N. Namboothiri, Int. J. Mass Spectrom., 2001, 204, 133-142.

17. E. C. Kempen and J. S. Brodbelt, Analytical Chemistry, 2000, 72, 5411-5416.

18. S. Blair, E. Kempen and J. Brodbelt, Journal of the American Society for Mass Spectrometry, 1998, 9, 1049-1059.

19. S. M. Blair, J. S. Brodbelt, A. P. Marchand, H. S. Chong and S. Alihodzic, Journal of the American Society for Mass Spectrometry, 2000, 11, 884-891. 
20. J. S. Brodbelt, E. Kempen and M. Reyzer, Structural Chemistry, 1999, 10, 213-220.

21. B. J. Goolsby, J. S. Brodbelt, E. Adou and M. Blanda, Int. J. Mass Spectrom., 1999, 193, 197-204.

22. E. C. Kempen and J. S. Brodbelt, Analytical Chemistry, 1999, 71, 5493-5500.

23. A. P. Marchand, H. S. Chong, K. A. Kumar, S. Alihodzic, S. M. Blair and J. S. Brodbelt, Abstracts of Papers of the American Chemical Society, 1999, 218, U1051-U1051.

24. S. M. Williams, J. S. Brodbelt and R. A. Bartsch, Journal of the American Society for Mass Spectrometry, 2003, 14, 1215-1228.

25. C. J. Pedersen, Chemica Scripta, 1988, 28, 229-235.

26. A. Boda, S. M. Ali, M. R. K. Shenoi, H. Rao and S. K. Ghosh, Journal of Molecular Modeling, 2011, 17, 1091-1108.

27. D. Schaumloffel and A. Tholey, Analytical and Bioanalytical Chemistry, 2011, 400, $1645-1652$.

28. M. J. Keith-Roach, Anal. Chim. Acta, 2010, 678, 140-148.

29. K. Wang and G. W. Gokel, The Journal of Organic Chemistry, 1996, 61, 4693-4697.

30. Periodic Table, http://www.rsc.org/periodic-table, (accessed December 15, 2016).

31. J. J. Manura, Isotope Distribution Calculator and Mass Spec Plotter, http://www.sisweb.com/mstools/isotope.htm).

32. G. Vanini, C. A. Destefani, B. B. Merlo, M. Carneiro, P. R. Filgueiras, R. J. Poppi and W. Romao, Microchemical Journal, 2015, 118, 19-25.

33. L. LaGoo, L. S. Schaeffer, D. W. Szymanski and R. W. Smith, J. Forensic Sci., 2010, 55, 624-632.

34. E. Diaz, J. E. Souza Sarkis, S. Viebig and P. Saldiva, Forensic Sci.Int., 214, 44-47.

35. A.-C. Lindström, J. Hoogewerff, J. Athens, Z. Obertova, W. Duncan, N. Waddell and J. Kieser, Forensic Sci.Int., 253, 103-111.

36. A. Santos, P. Ramos, L. Fernandes, T. Magalhães, A. Almeida and A. Sousa, Forensic Sci.Int., 247, 62-68.

37. A. Santos, T. Magalhaes, D. N. Vieira, A. A. Almeida and A. V. Sousa, Am. J. Forensic Med. Pathol., 2007, 28, 24-30.

38. R. G. Vibhute and S. M. Khopkar, Talanta, 1989, 36, 957-959. 
39. A. Guy, P. Jones and S. J. Hill, Analyst, 1998, 123, 1513-1518.

40. D. R. Lide, ed., CRC Handbook of Chemistry and Physics, CRC Press, 2000.

41. A. P. Marchand, J. S. Brodbelt and K. Mlinaric-Majerski, ChemInform, 2005, 36, no-no.

42. S. M. Blair, J. S. Brodbelt, A. P. Marchand, K. A. Kumar and H. S. Chong, Analytical Chemistry, 2000, 72, 2433-2445.

43. R. M. Izatt, K. Pawlak, J. S. Bradshaw and R. L. Bruening, Chemical Reviews, 1991, 91, 1721-2085.

44. S. M. WIlliams, Doctor of Philosophy, The University of Texas at Austin, 2003. 


\section{Chapter 5: Conclusions and Future Directions}

\subsection{Conclusions}

Modernizing the analysis of firearm discharge residue plays a key role in the ability to increase its evidentiary value. The methods presented are advanced analytical techniques. Firearm discharge residue studies began in our laboratory with investigating the persistence and permeation of OGSR compounds. Ion mobility spectrometry proved fit for purpose during these experiments and therefore a valuable tool for detecting OGSR. The initial studies presented here evaluated and validated IMS for OGSR compounds. In addition to validation, IMS showed promise in a population study as a screening method for organic gunshot residues on hand swabs. The results argue for a pattern-based analysis rather than relying on peak identification for characterizing shooters vs. non-shooters hand swabs. In addition, control charts plotting a daily standard allowed instrument performance to be tracked during the lifetime of the project.

Due to the effectiveness of thermal desorption as a means of sample introduction with IMS and the need to reach lower limits of detection, a thermal separation probe was evaluated as a means of sample introduction for OGSR analysis with GC/MS. TSP GC/MS eliminates the need for sample preparation and pre-concentration steps. Characterizing surrogate compound results via bivariate plots provided means to assign probabilities to combinations of ion ratios and retention time data and to establish acceptance criteria. Concerns arose with the detection of compounds in blank swabs and the way in which the swabs were loaded into the TSP.

Finally, focus was shifted to investigating a method with the ability to detect both components of FDR. Through complexation with crown ethers, common GSR metals were detectable via ESI/MS ${ }^{\mathrm{n}}$, and instrument typically reserved for organic molecules. Single and double ligand complexes were identified and mass spectral intensities were used to calculate 
binding selectivities. Experimentally calculated binding selectivities agreed well with theoretical values calculated via MINEQL+ Chemical Equilibrium Modeling System software. In addition, molecular modeling results were able to provide insight into the inability to detect antimony and provided a reliable means for screening additional complexing agents.

\subsection{Future Directions}

Additional work is needed prior to implementing the methods discussed here into forensic laboratories. Work is already underway in our laboratory on advancing collection methods and detection with TSP-GC/MS. The TSP-GC/MS was recently validated, addressing the concerns previously mentioned; additional qualifier ions were added to the SIM method and the glass microvial that previously held the swab was eliminated. The additional qualifier ions allowed for increased accuracy in compound identification and the elimination of the glass microvial provided better air flow and more efficient heating during sample introduction. This validation study is currently in the publication process.

Several projects could build on the research discussed here. Figure 5.1 depicts the projected next phase of the research presented as research migrates towards the application of the techniques discussed towards authentic FDR sample. First, collection methods must be readdressed and re-evaluated. For the techniques discussed and for any FDR detection method to be practical for forensic use, it must be capable of detecting residues at forensically relevant amounts, such as that as a result of 1-3 shots. To reach these levels it is crucial to improve the recovery of residues during the collection and extraction processes. 


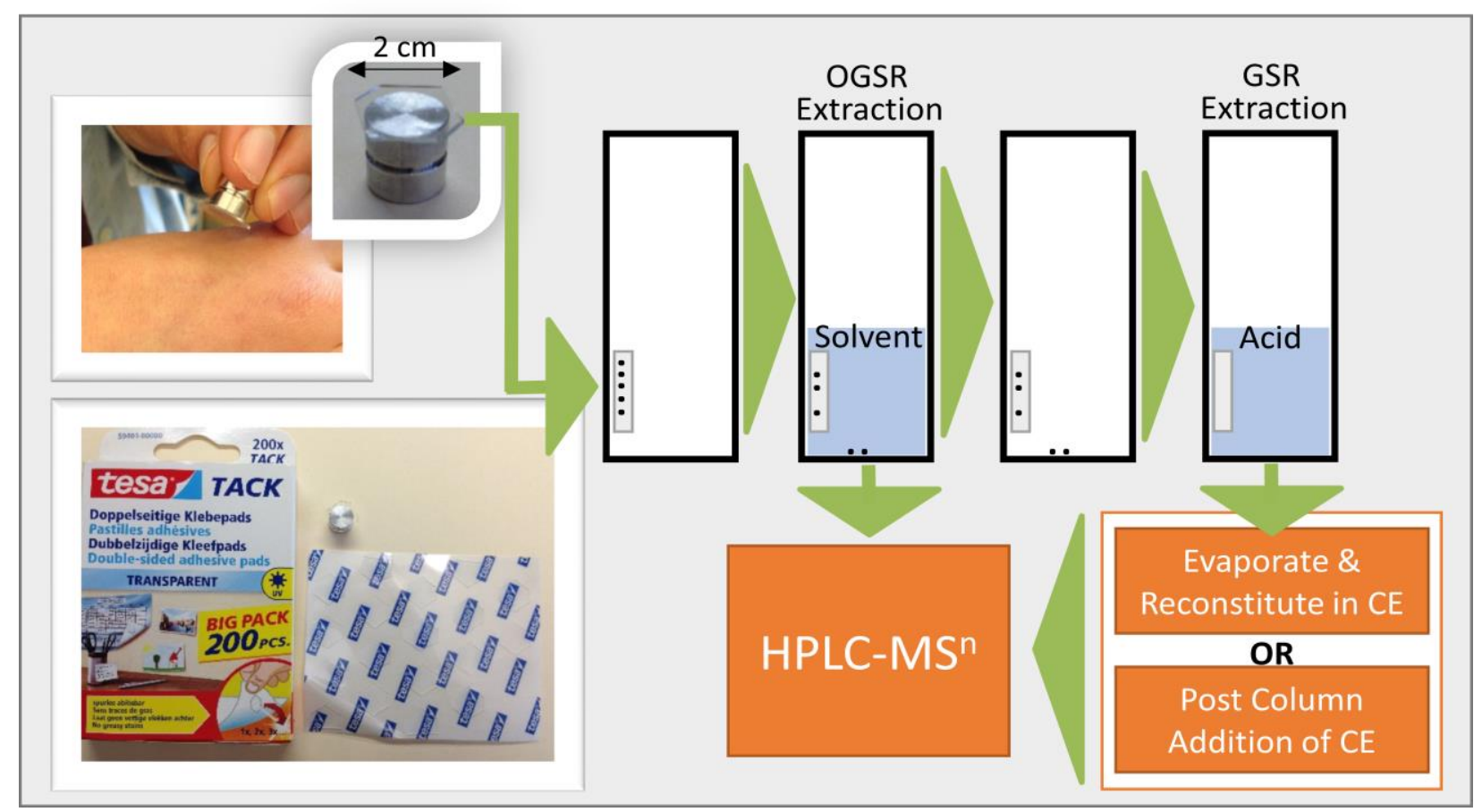

Figure 5.1 Depiction of the next phase of FDR research in our laboratory.

A suggested material for collection is an international consumer product called Tesa ${ }^{\circledR}$ Tack. It is a transparent double sided adhesive pad with a surface area of approximately $4 \mathrm{~cm}^{2}$ commonly used to adhere paper materials to walls and similar surfaces. The adhesion allows for the collection of GSR and OGSR while the relatively small surface area allows for sample preconcentration. Preliminary experiments have been performed utilizing Tesa ${ }^{\circledR}$ Tack during sample collection. For collection, one tack square is placed on the end of a stainless steel stub, typically used in the collection of GSR particles, and is dabbed across the hands. Tessa® Tack appeared easy to use as no pre-wetting or additional preparation was required prior to use. Recovery studies would need performed and concerns exist about the degradation of the collection material during compound extraction.

For extraction, solubility of all components must be considered and issues with antimony must be further understood and addressed. The current extraction protocol is a sequential 
multistep process utilizing multiple solvents; methanol for the organic compounds and nitric acid for the inorganic metals. Another extraction method to be evaluated is a multiphase extraction. The sequential extraction produces one solution containing both OGSR and GSR where the multiphase extraction would separate OGSR and GSR to produce two solutions. The location in the process in which crown ether or any other host molecule is introduced is also a factor to be explored. There are three locations for this to occur: (1) during the extraction process, (2) after extraction before analysis, or (3) post-column.

Furthermore, the binding selectivity results presented and observations during ESI-MS analysis indicates that target metals must be separated from contaminates, such as potassium and sodium, prior to analysis. Potassium and sodium along with other contaminants could come from the skin during collection, the solvents or even the glassware used in extraction and analysis. Two issues with the contaminants are prevalent; (1) competition for host and (2) signal suppression. Experiments determined that the crown ether used (15-crown-5) preferentially bound potassium. Therefore, if a competitive environment for crown ether exists, such as too little crown ether, potassium ions would cause the other metals to unbind. Additionally, the use of excess crown ether could result in signal suppression either by the crown ether or by the increased signal of complexed potassium. In both instances, vital information is lost and would produce a false negative result. Care was taken during the experiments presented to minimize contaminant levels such as using polished water and plastic ware for making and storing solutions. In the future, utilizing a desalting column during the extraction process or during separation by using a cation exchange guard column are potential options to be explored.

The future of firearm discharge residue evidence lies in the application of modern instruments and technology. Although further evaluation is recommended, the methods 
discussed have demonstrated that they are fit for purpose. While IMS and TD-GC/MS proved valuable as screening tool for OGSR, it is evident that to increase the evidentiary value samples must be analyzed for both GSR and OGSR. Utilizing the methods of complexing for metal ions and ESI-MS ${ }^{\mathrm{n}}$ for detection, as discussed here, allows for the dual detection of both components. The key to this method being successful lies in the pre-analysis steps, such as collection and extraction. 


\section{Appendix A}

\section{Chapter 2: Evaluation and validation of Ion Mobility Spectrometry for Presumptive Testing Targeting the Organic Constituents of Firearms Discharge Residue - Supplemental Information}

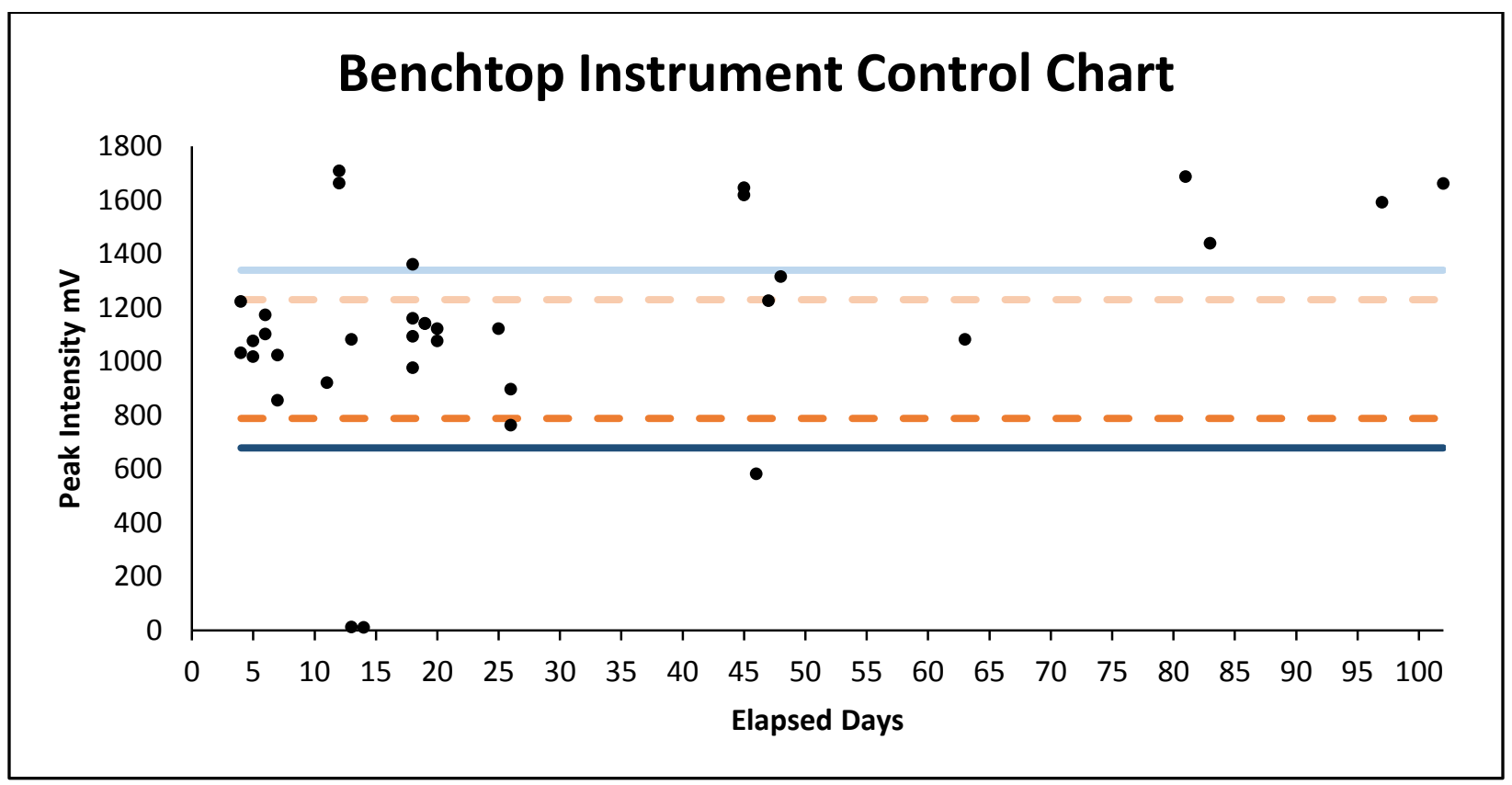

Figure A1. Control chart for the benchtop instrument.

As noted in the article, the performance of this instrument over time indicated a greater variation and degradation compared to the benchtop. Depending on the analysis being done, data was still collected but was flagged for further consideration and scrutiny. Note that on several days, multiple DtBP samples were collected as the instrument was used both morning and afternoon. Intra-day variation was greater with this instrument than with the portable.

It is worth noting that even on days when the warning and control limits were exceeded, the instrument was still operational and would have passed instrument verification challenges. 
The DtBP was purposely selected to provide additional quality assurance that clearly will be essential if this methodology is to be adapted for screening purposes.

Figures A2-A5. Detection threshold plots, benchtop instrument

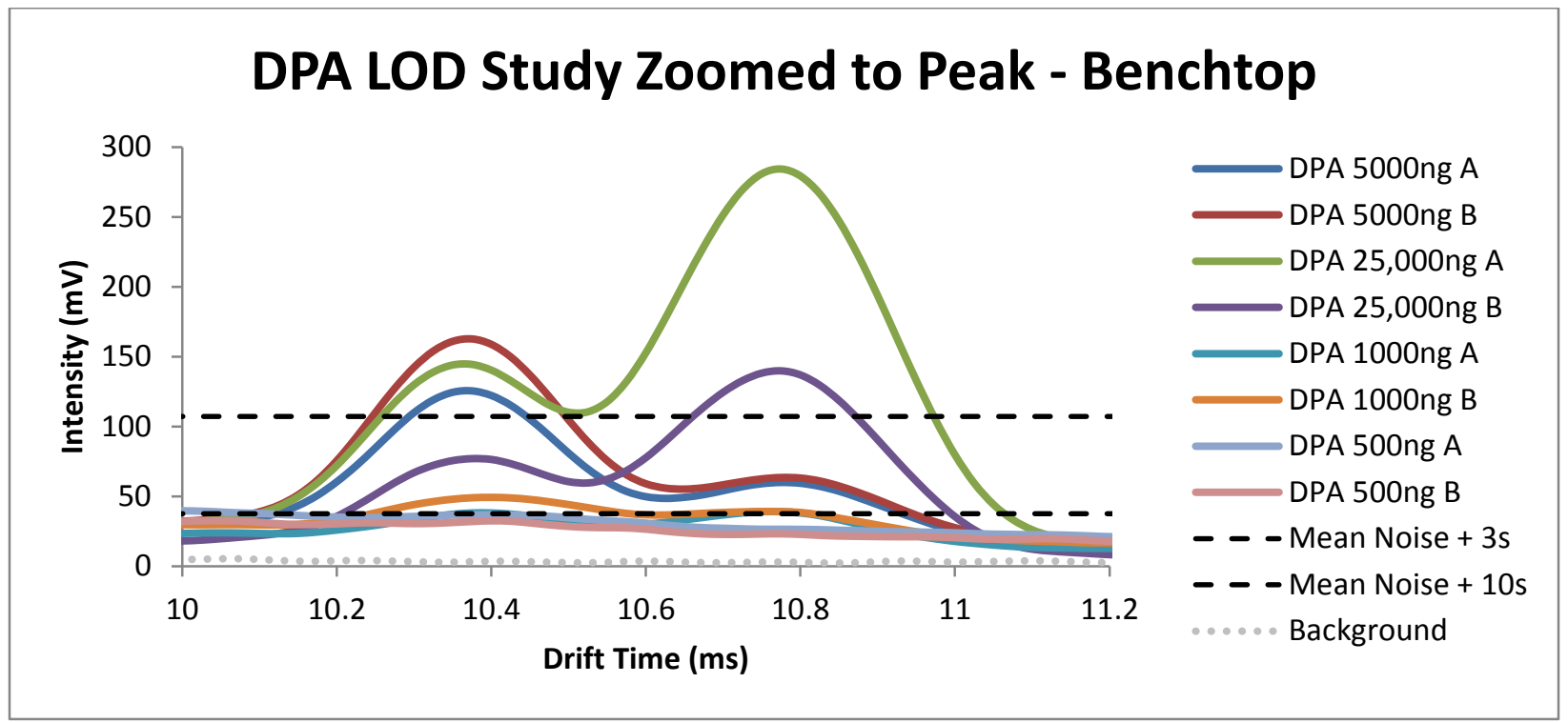

Figure A2. Dimethyl phthalate spectra ranging from 1ng to 25,000ng in which the detection threshold was determined to be 500ng for the benchtop instrument.

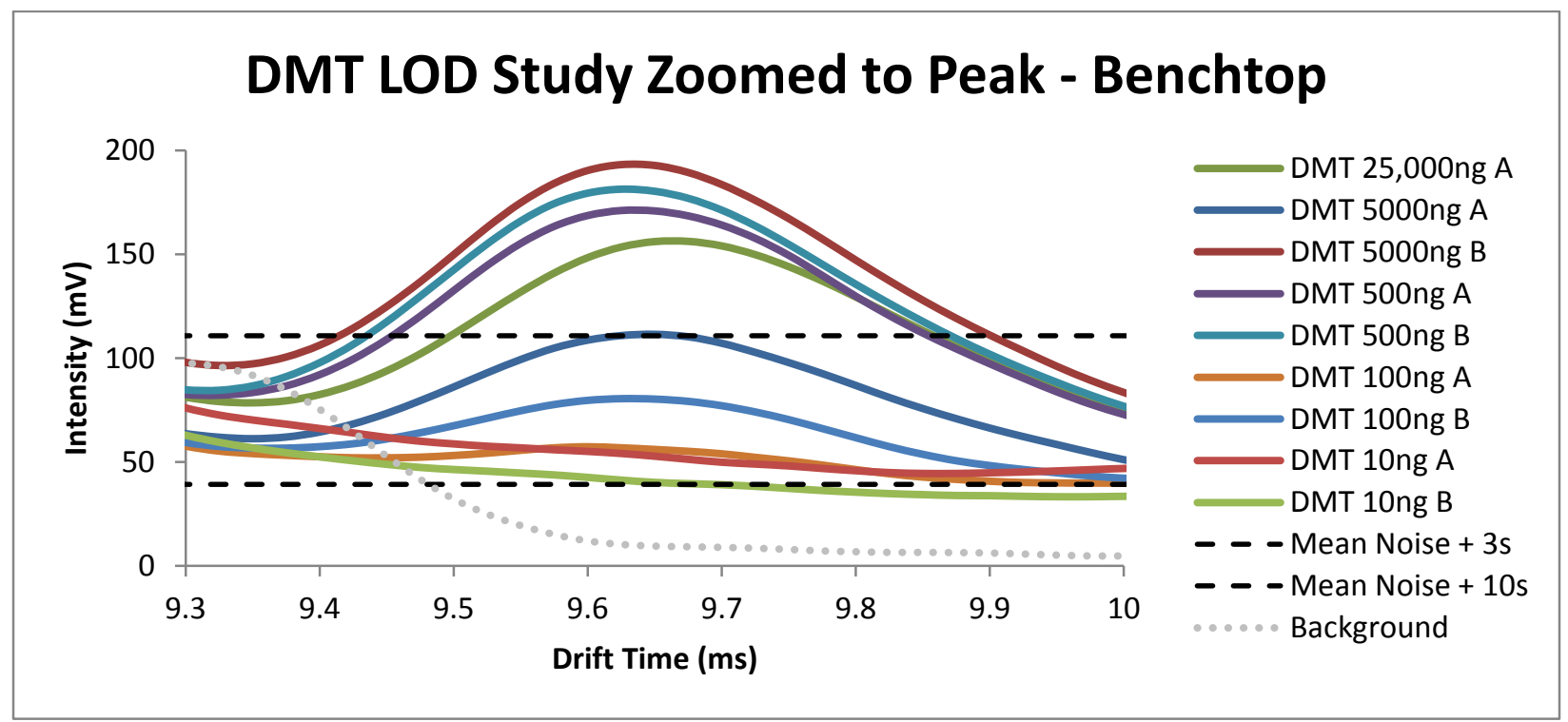

Figure A3. Diphenylamine spectra ranging from 500ng to 25,000ng in which the detection threshold was determined to be 1000ng for the benchtop instrument. 
Note that two peaks are associated with DPA (Figure S3). This presumably arises from thermal degradation of DPA although the product(s) is unknown. The benchtop instrument was operated at higher temperatures than the portable (Table 2) for reasons discussed in the text; however for DPA the hotter temperatures appear to be less desirable for peak-based detection. With a pattern-based approach, the higher temperatures may not be a limitation.

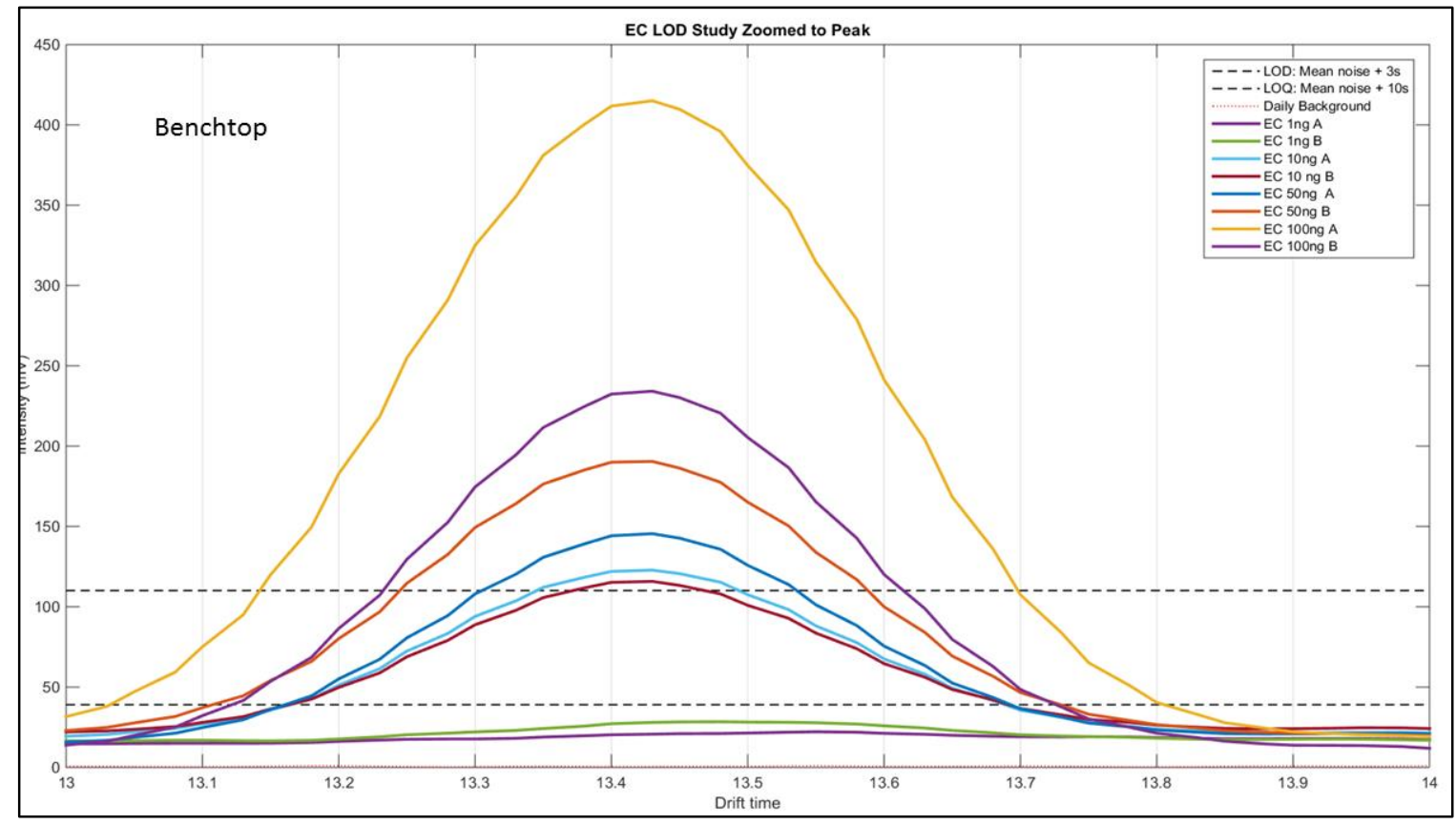

Figure A4. Ethyl centralite spectra ranging from 1ng to 100ng in which the detection threshold was determined to be 10ng for the benchtop instrument. 


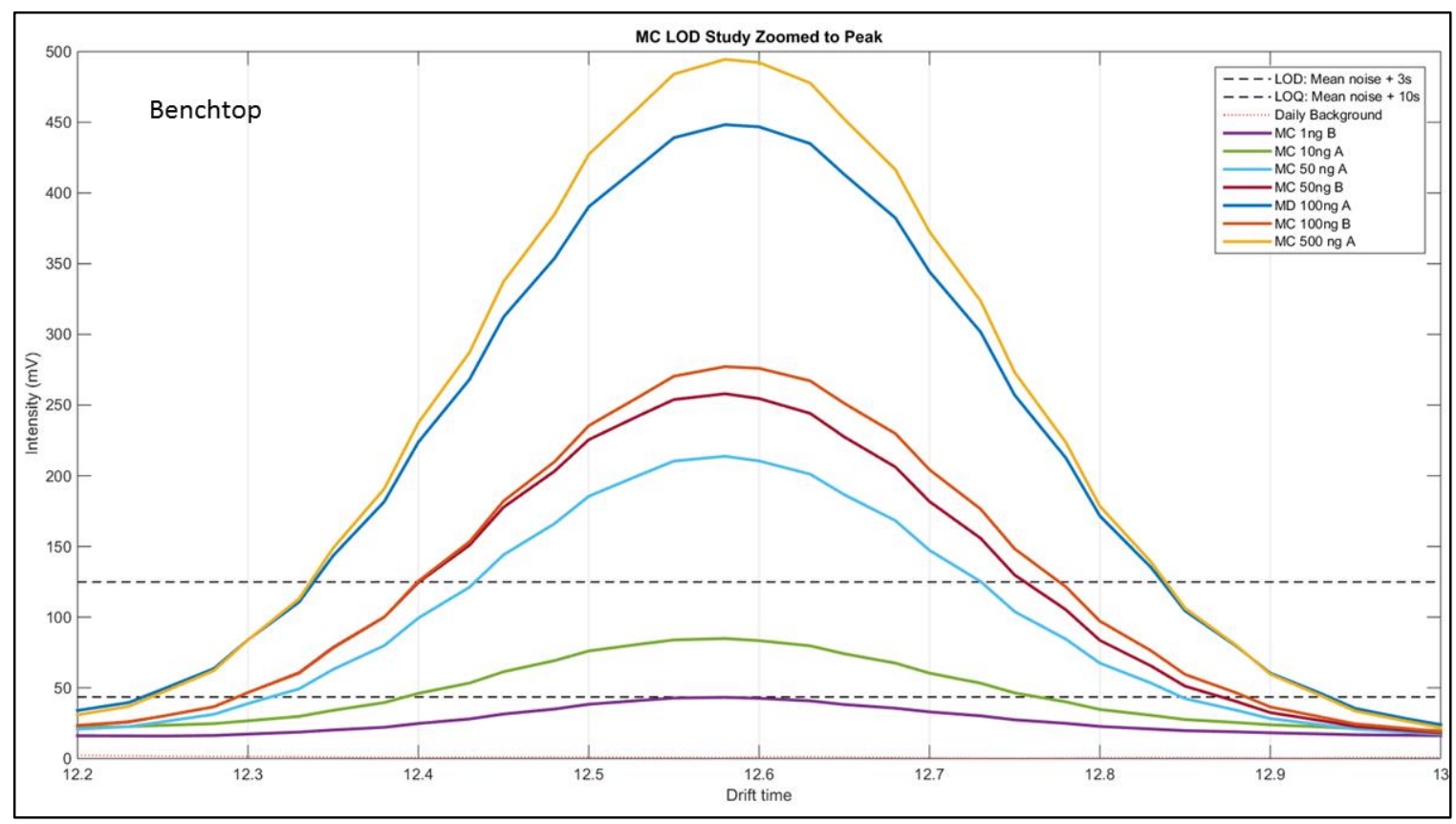

Figure A5 Methyl centralite spectra ranging from 1ng to 500ng in which the detection threshold was determined to be $10 \mathrm{ng}$ for the benchtop instrument.

Figures A6-A9. Detection threshold plots, handheld instrument

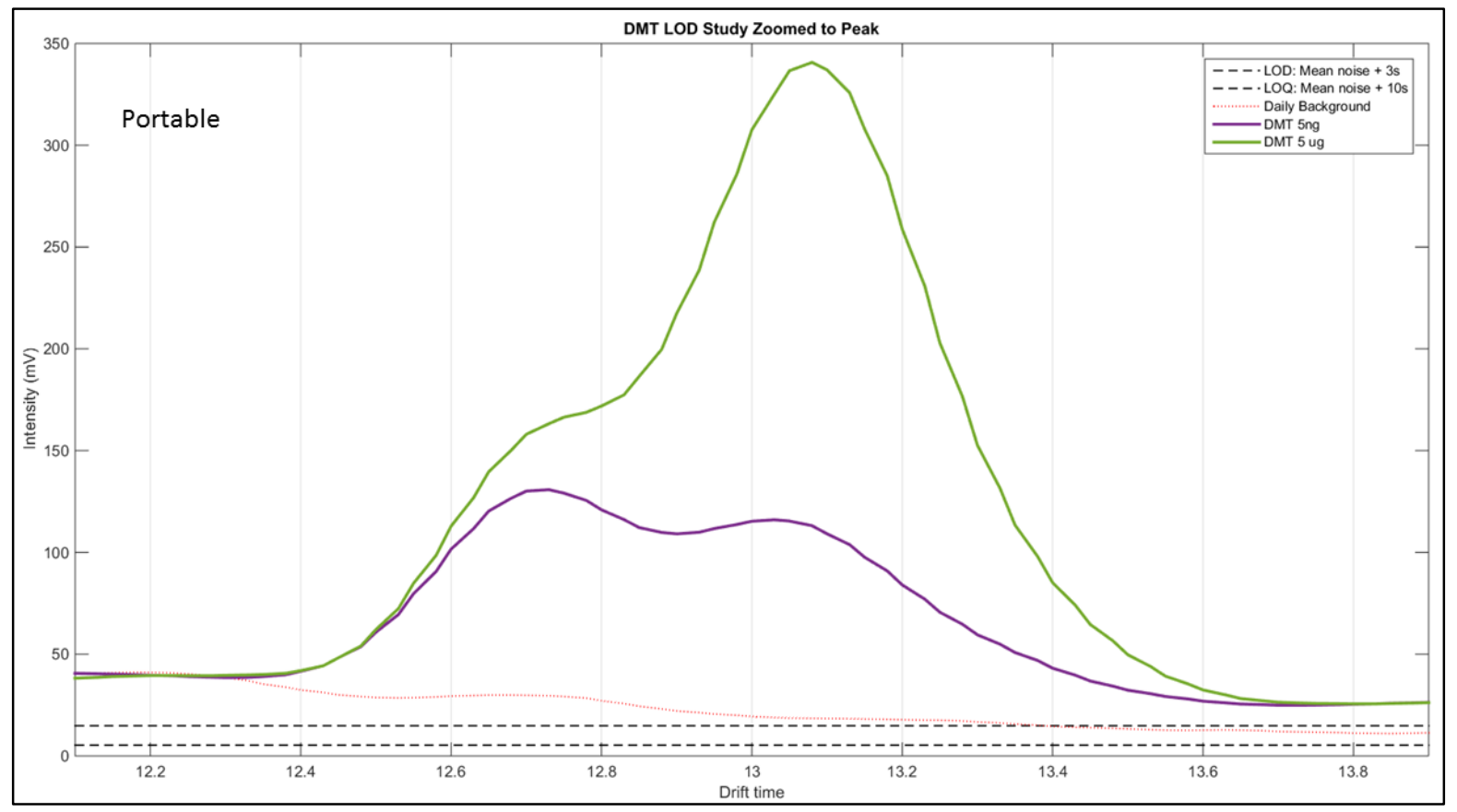

Figure A6. Dimethyl phthalate spectra in which the detection threshold was determined to be 5ng for the portable instrument. The width of the peak may be attributable to clustering. For the benchtop instrument (Figure S2, higher temperatures) this pattern was not observed. 


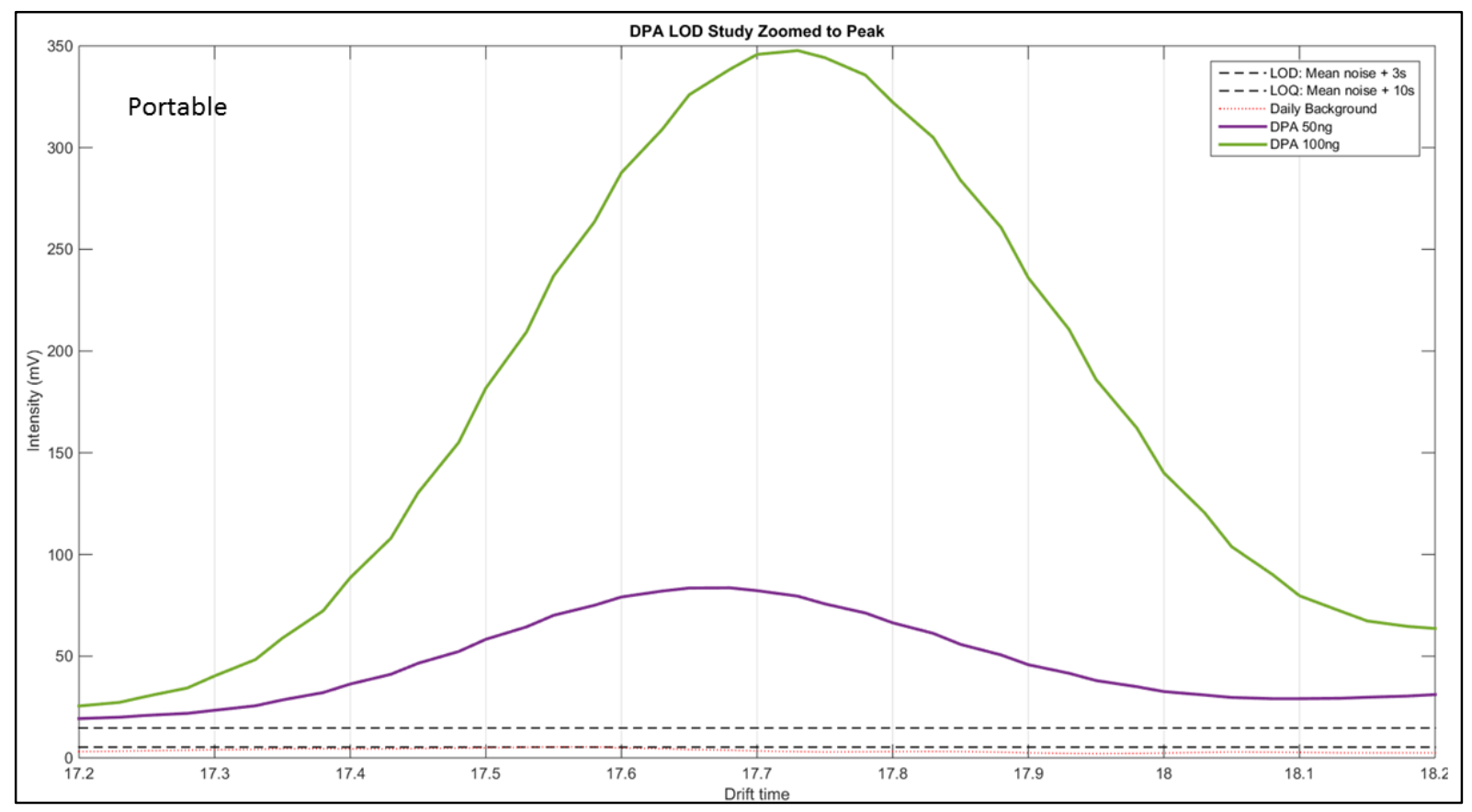

Figure A7. Diphenylamine spectra in which the detection threshold was determined to be 50ng for the portable instrument.

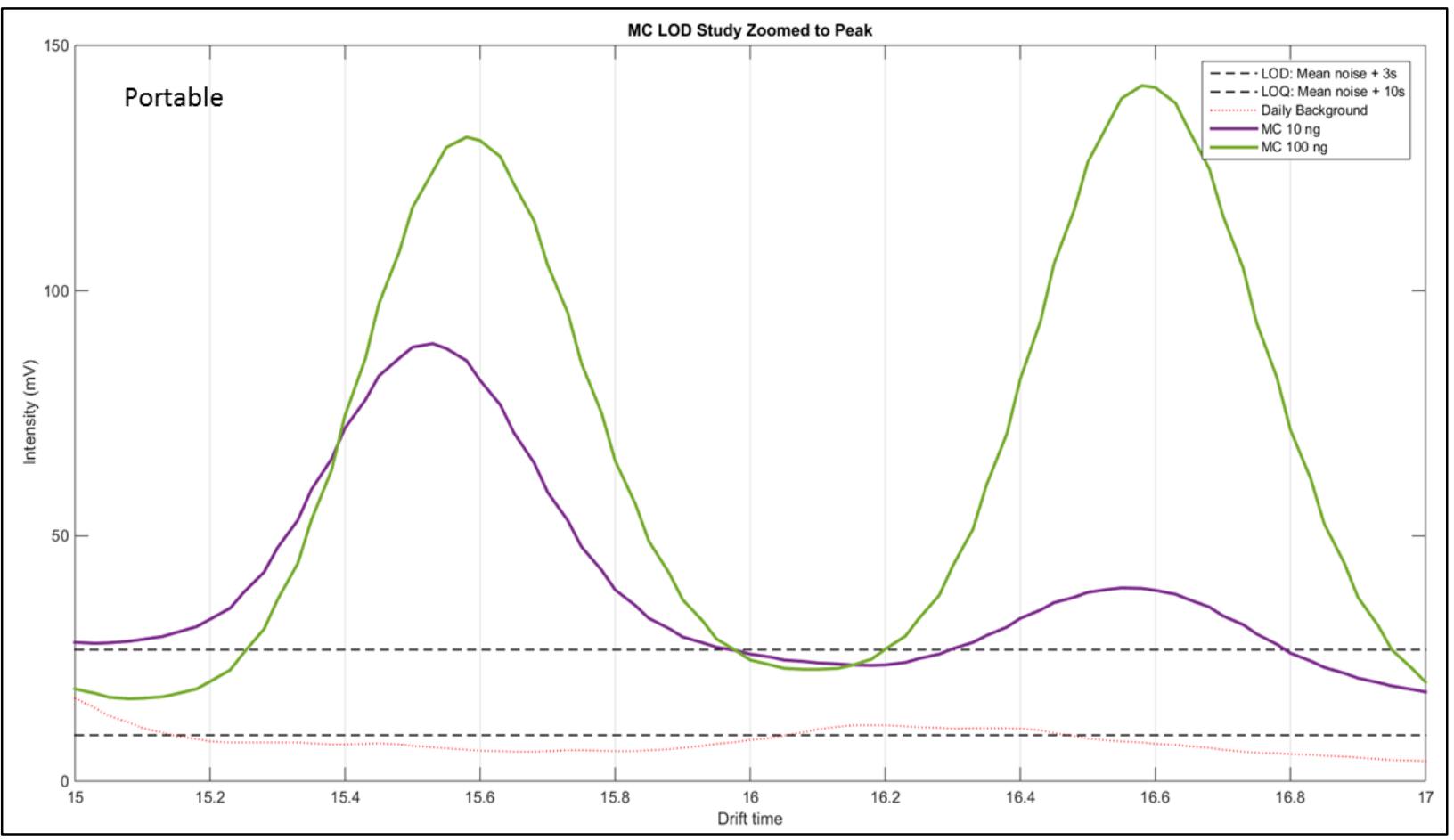

Figure A8. Methyl centralite spectra in which the detection threshold was determined to be 10ng for the portable instrument. The appearance of two mobility peaks was seen in the portable but not with the benchtop (Figure 2.3 in the text). This could represent a monomer/dimer pair but this is unconfirmed. 


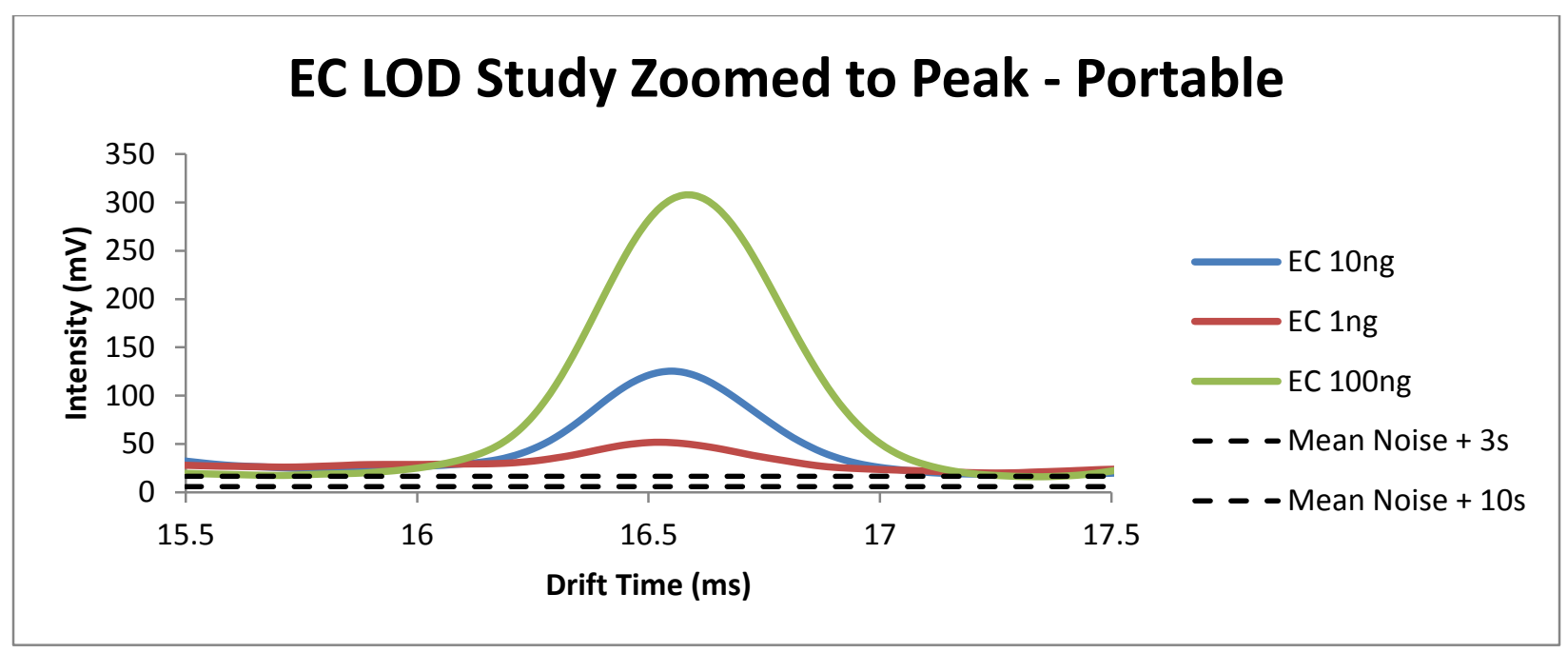

Figure A9. Ethyl centralite spectra in which the detection threshold was determined to be 1ng for the portable instrument.

\section{Appendix B}




\section{Chapter 3: Initial evaluation of inlet thermal desorption GC-MS analysis for organic gunshot residue collected from the hands of known shooters. - Supplemental Information}

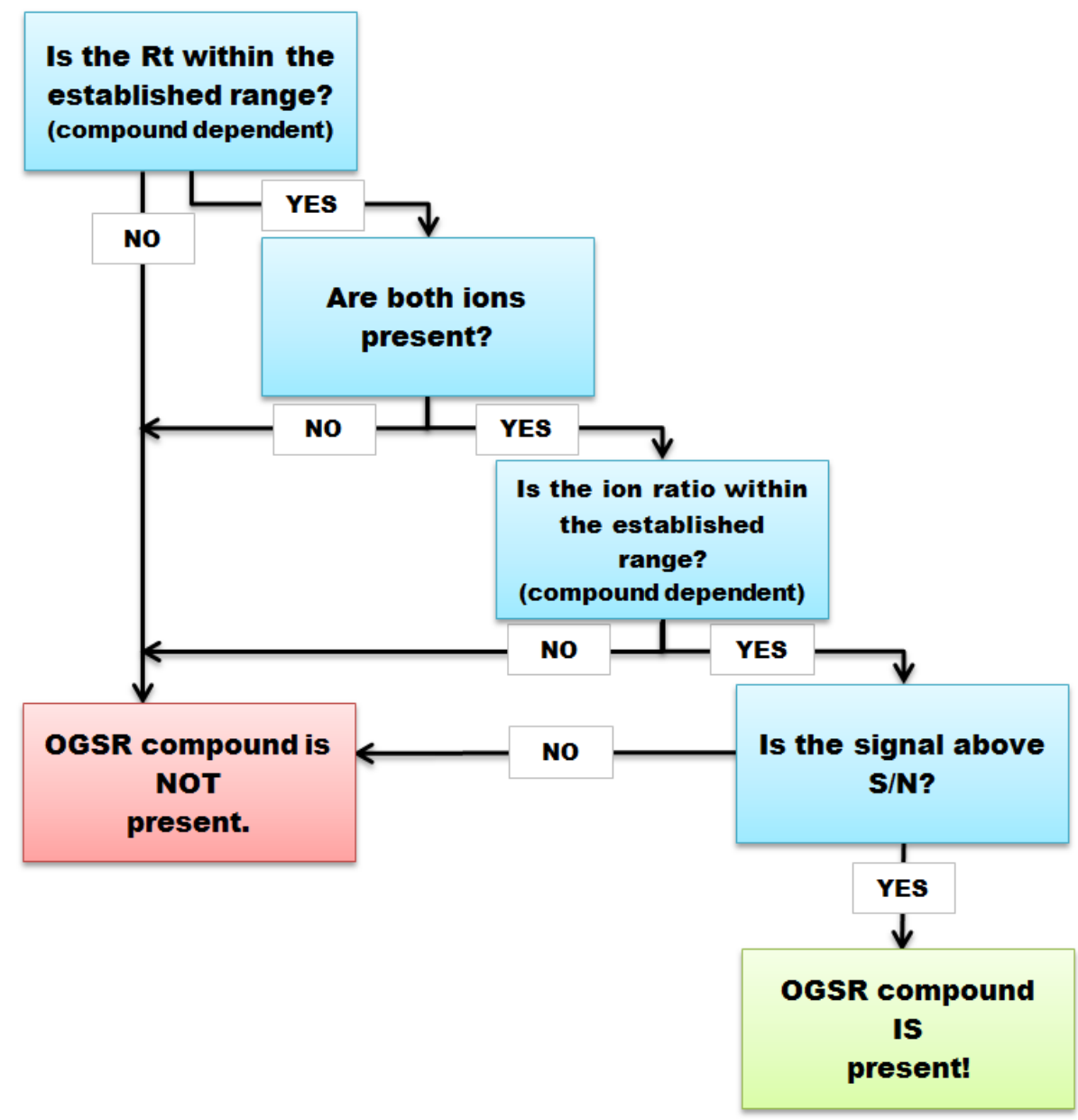

Figure B3.1 Flowchart used to determine the presence of OGSR compounds in authentic shooting samples.

Table B3.1 Percent RSD for criteria data. 


\begin{tabular}{|l|c|c|}
\hline & RT & Ratio \% \\
\hline DMP & 0.11 & 38.14 \\
\hline 2,4-DNT & 0.11 & 10.70 \\
\hline DPA & 0.09 & 36.13 \\
\hline MC & 0.10 & 11.28 \\
\hline Carbazole & 0.10 & 31.18 \\
\hline EC & 0.11 & 17.11 \\
\hline DBP & 0.10 & 54.27 \\
\hline 2-NDPA & 0.08 & 8.21 \\
\hline 4-NDPA & 0.20 & 10.56 \\
\hline
\end{tabular}

Table B3.2 Example criteria data for DPA.

\begin{tabular}{|c|c|c|c|c|c|}
\hline File & $\begin{array}{l}\text { Quantifier } \\
\text { Ion RT } \\
(169.2) \\
\end{array}$ & $\begin{array}{l}\text { Qualifier Ion } \\
\text { RT (83.6) }\end{array}$ & $\begin{array}{l}\text { Quantifier } \\
\text { Ion Height }\end{array}$ & $\begin{array}{l}\text { Qualifier Ion } \\
\text { Height }\end{array}$ & $\begin{array}{l}\text { \% Qual. to } \\
\text { Quantifier } \\
\text { Ratio } \\
\end{array}$ \\
\hline File 1 & 14.87 & 14.87 & $8.01 \mathrm{E} 06$ & 2.09E06 & 26.0 \\
\hline File 2 & 14.84 & 14.84 & $1.91 \mathrm{E} 06$ & $3.55 \mathrm{E} 05$ & 18.6 \\
\hline File 3 & 14.84 & 14.84 & $6.23 \mathrm{E} 06$ & 1.47E06 & 23.7 \\
\hline File 4 & 14.84 & 14.84 & 2.74E06 & 4.71E05 & 17.2 \\
\hline File 5 & 14.84 & 14.84 & 7.51E04 & $1.36 \mathrm{E} 04$ & 18.2 \\
\hline File 6 & 14.84 & 14.84 & $3.06 \mathrm{E} 04$ & $5.42 \mathrm{E} 03$ & 17.7 \\
\hline Average & \multicolumn{2}{|c|}{14.831} & \multicolumn{3}{|c|}{21.848} \\
\hline Std. Dev. & \multicolumn{2}{|c|}{0.0135} & \multicolumn{3}{|c|}{7.893} \\
\hline
\end{tabular}




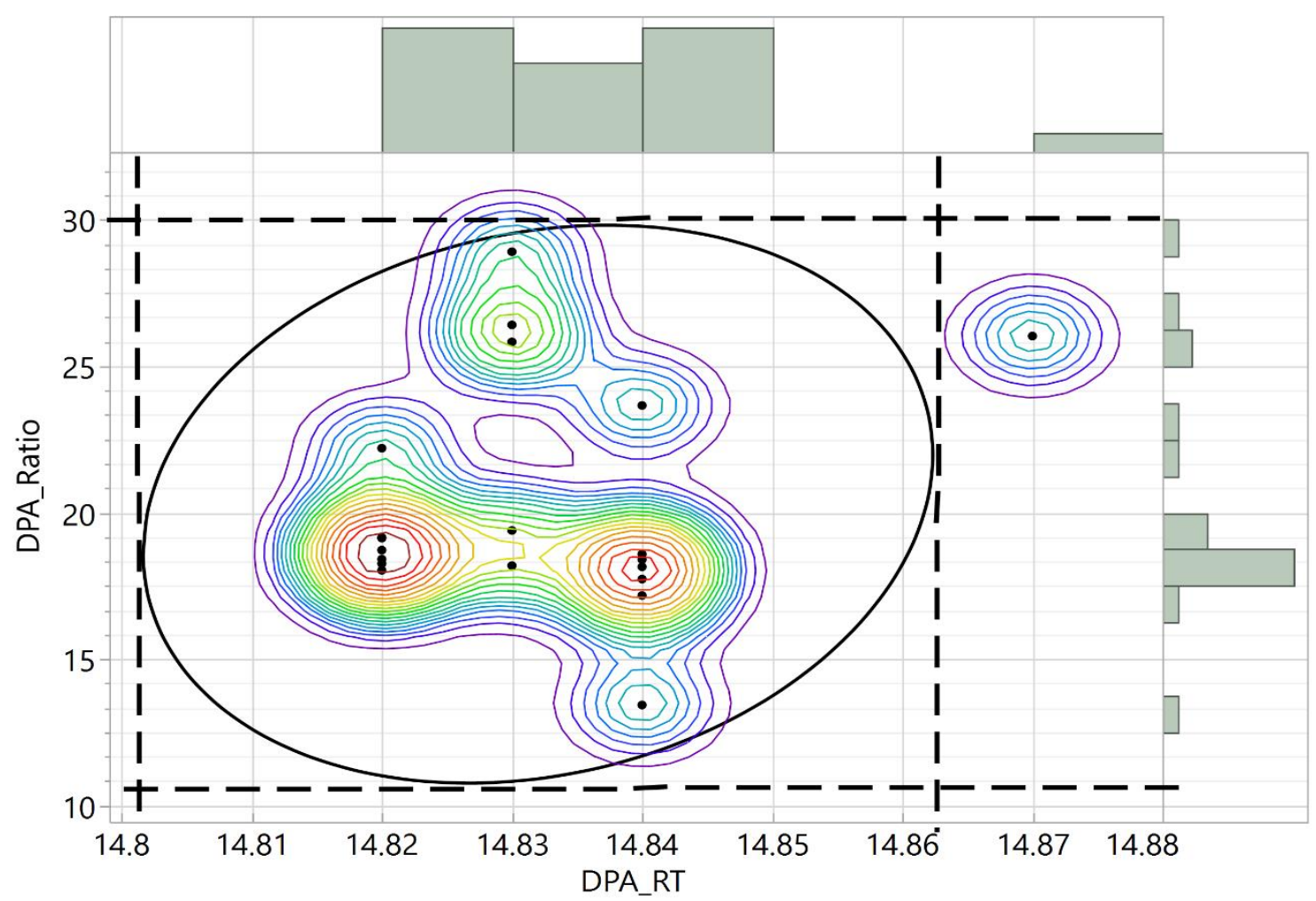

Figure B3.2a DPA bivariate plot with histograms $(\mathrm{n}=20)$.

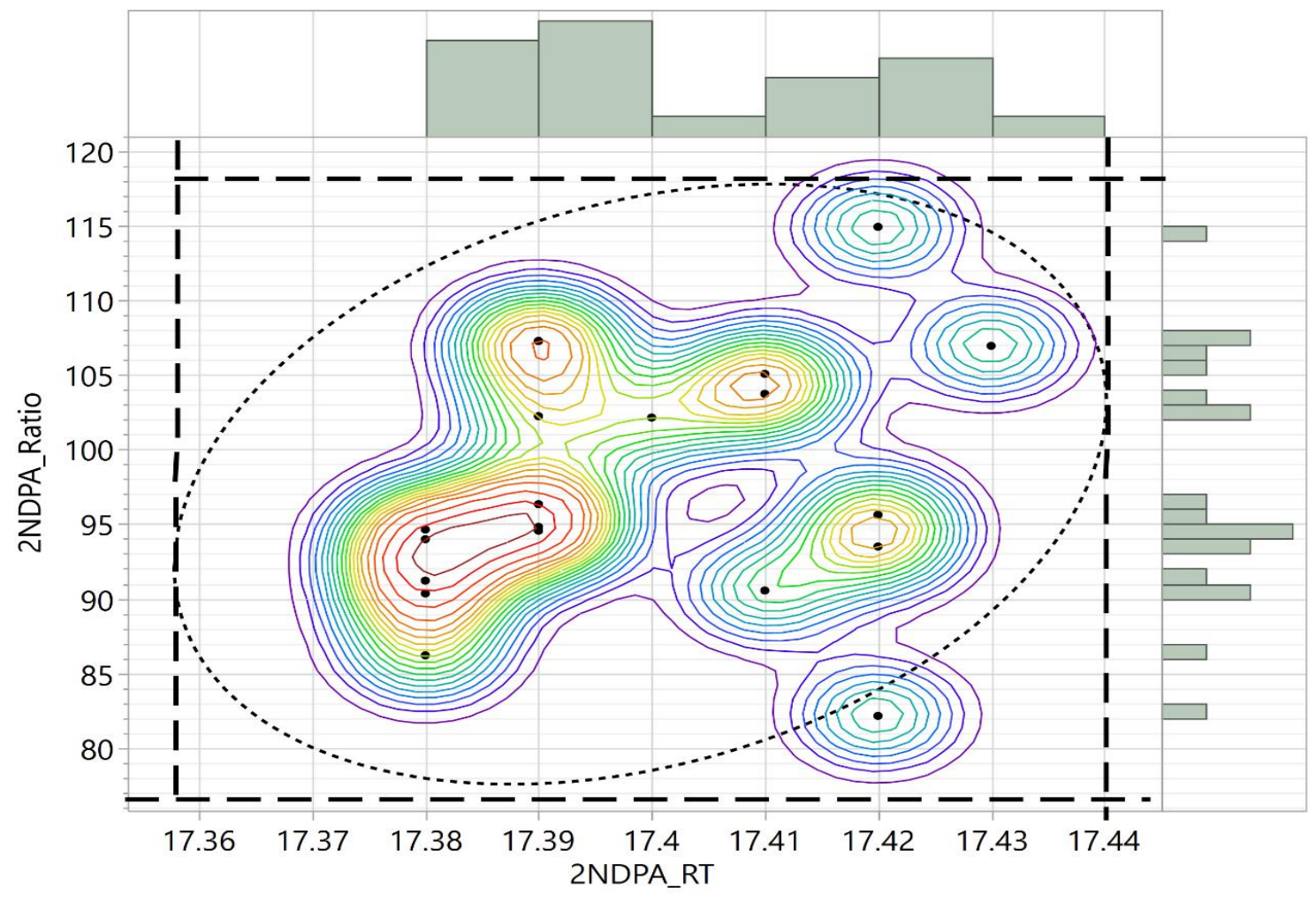

Figure B3.2b 2-NDPA bivariate plot with histograms $(n=21)$. 


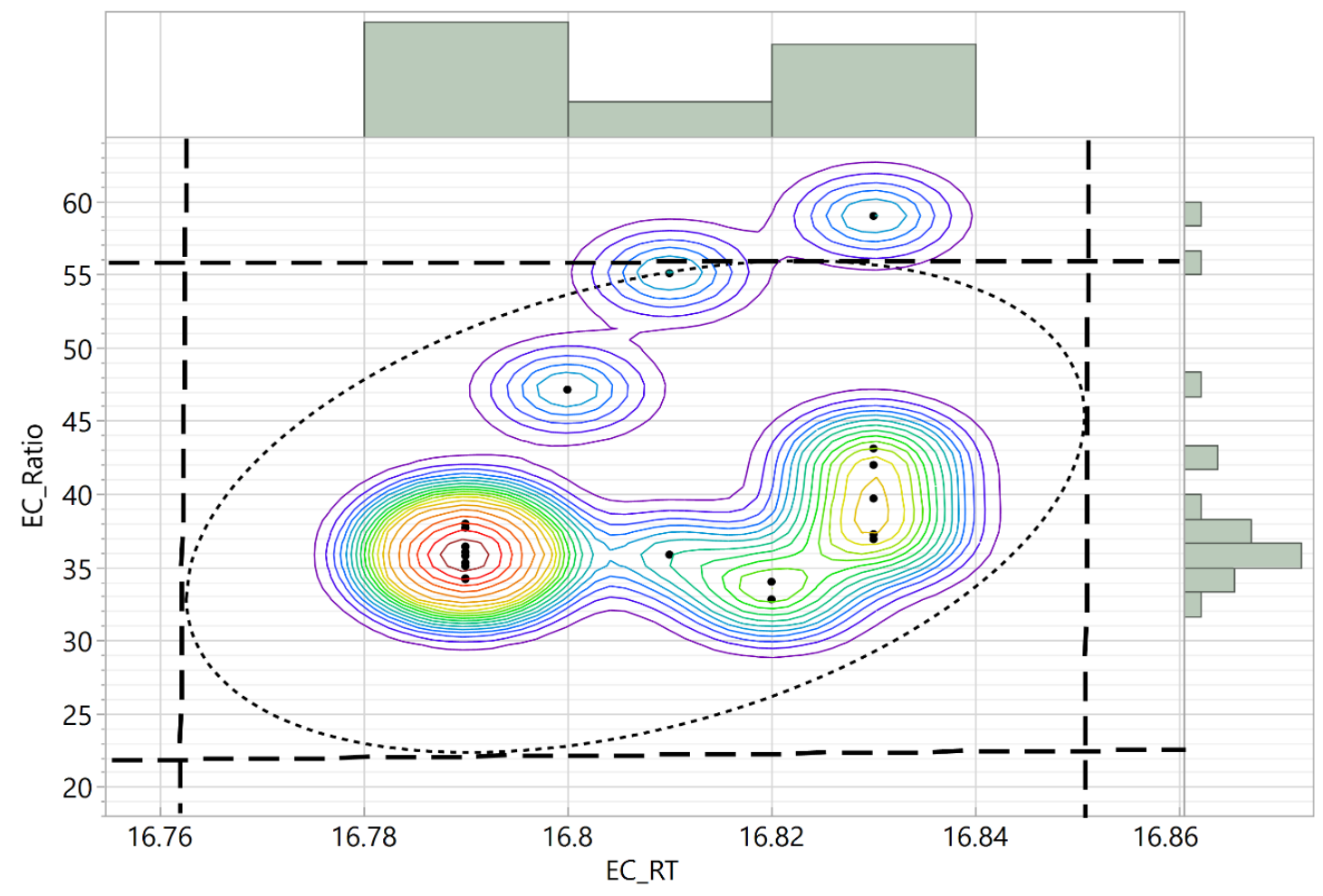

Figure B3.2c EC bivariate plot with histograms $(n=20)$. 


\section{Appendix C}

Chapter 3: Initial evaluation of inlet thermal desorption GC-MS analysis for organic gunshot residue collected from the hands of known shooters. - Supplemental Information

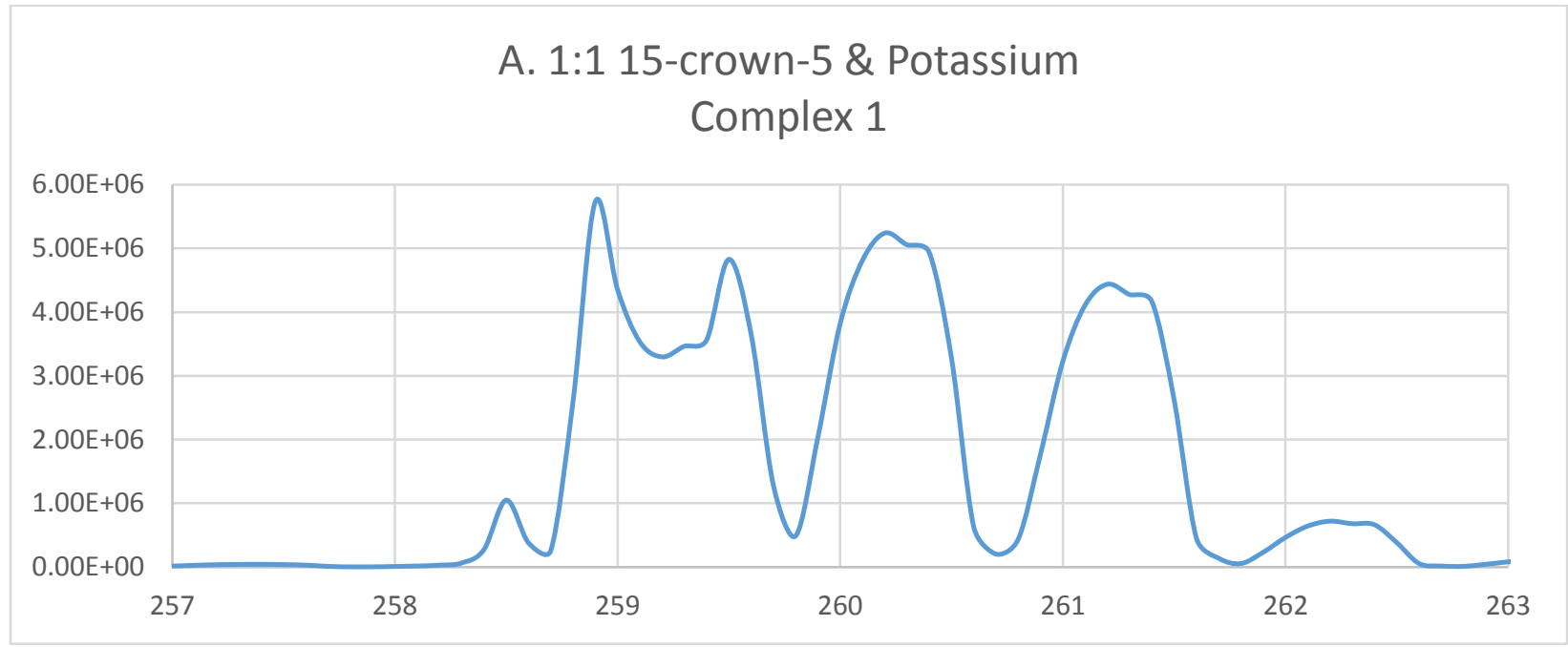

B. 1:1 15-crown-5 \& Potassium

Complex 2

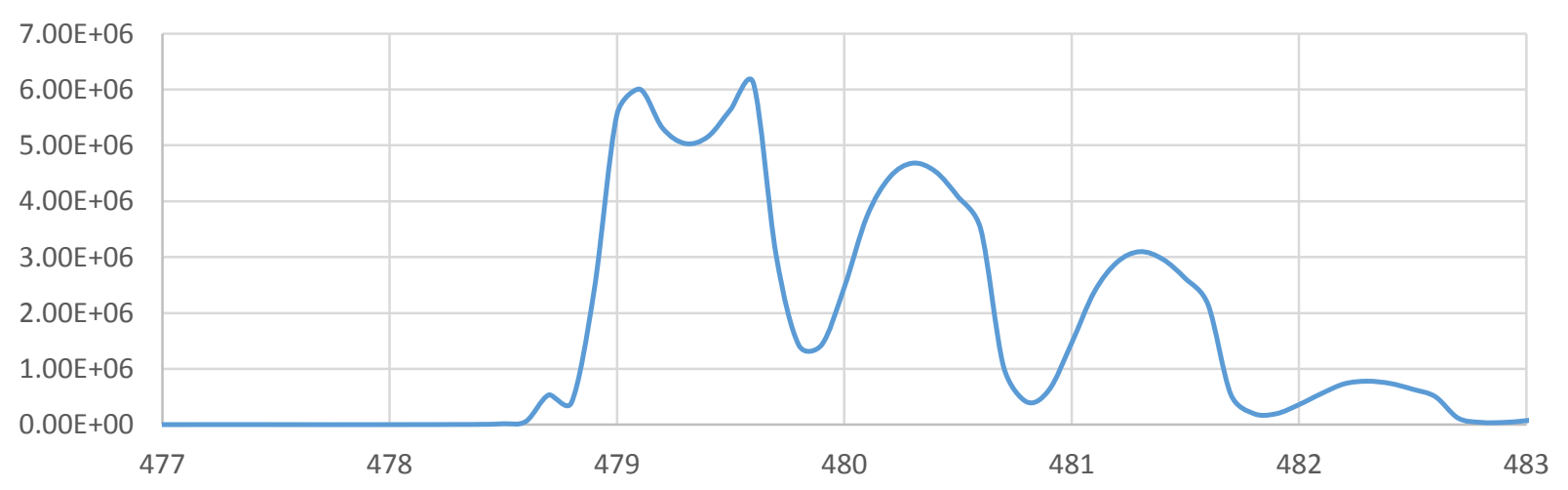

Figure C4.1 A \& B Saturated potassium complex peaks $\left(1.5 \times 10^{-4} \mathrm{M}\right)$. 


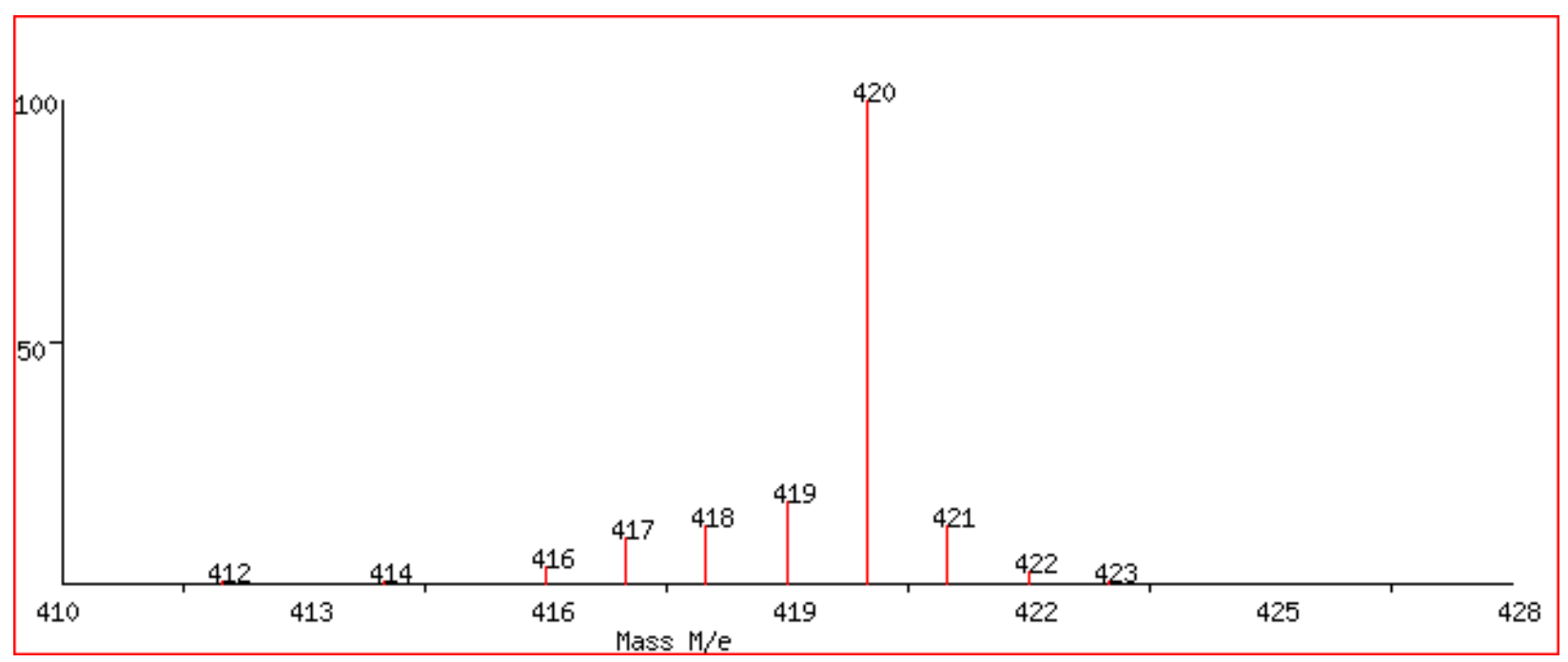

A. $\mathrm{C}_{10} \mathrm{H}_{20} \mathrm{O}_{5} \mathrm{BaNO}_{3}$

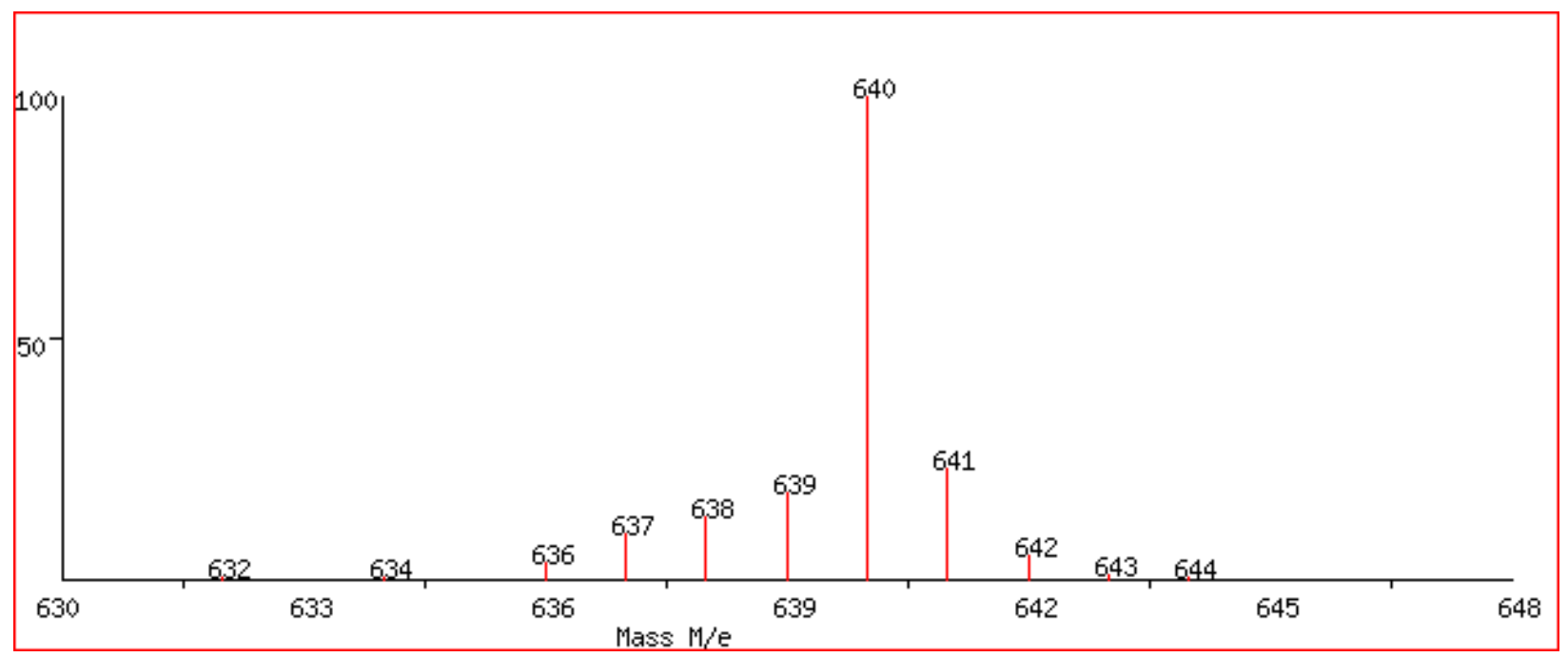

B. $\mathrm{C}_{20} \mathrm{H}_{40} \mathrm{O}_{10} \mathrm{BaNO}_{3}$

Figure C4.2 A-H Spectra generated from the Scientific Instrument Services, Inc. Isotope Distribution Calculator and Mass Spec Plotter used for the comparison of isotopic signatures in experimental spectra. 


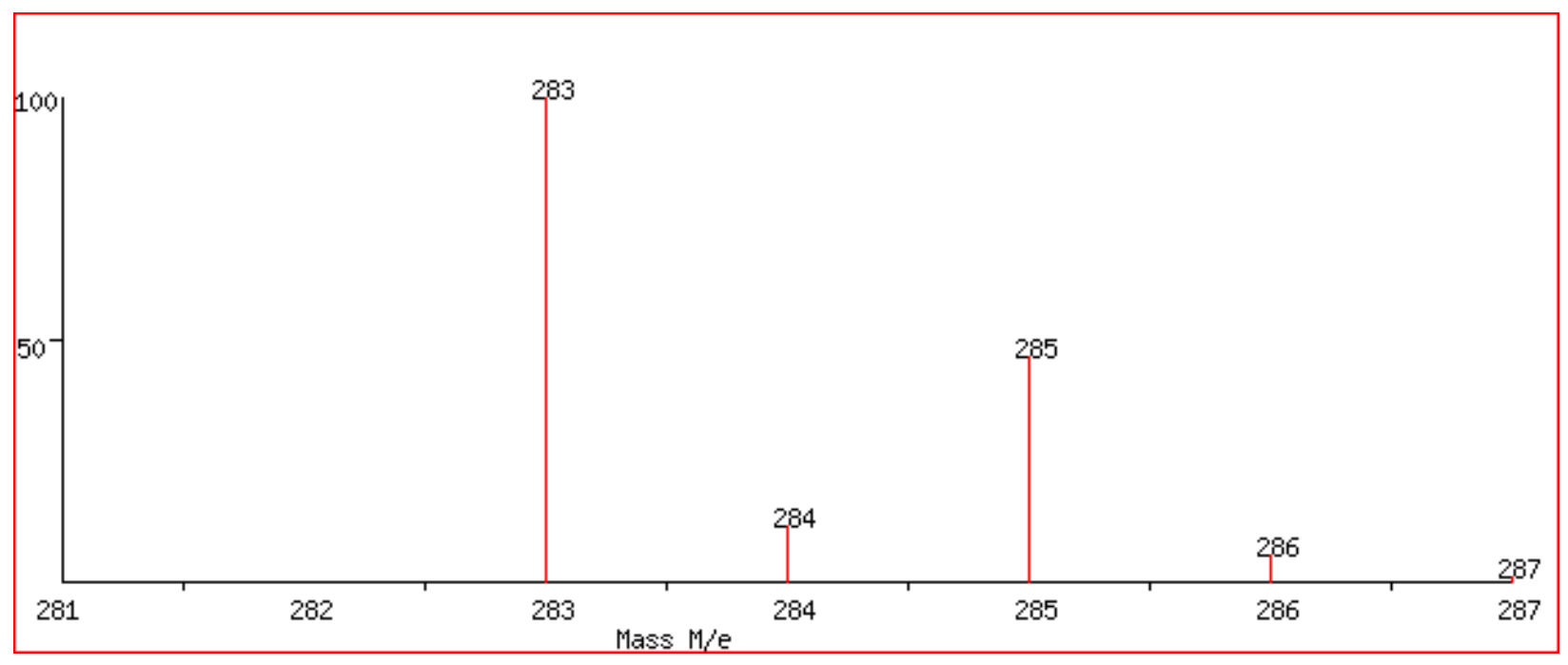

C. $\mathrm{C}_{10} \mathrm{H}_{20} \mathrm{O}_{5} \mathrm{Cu}$

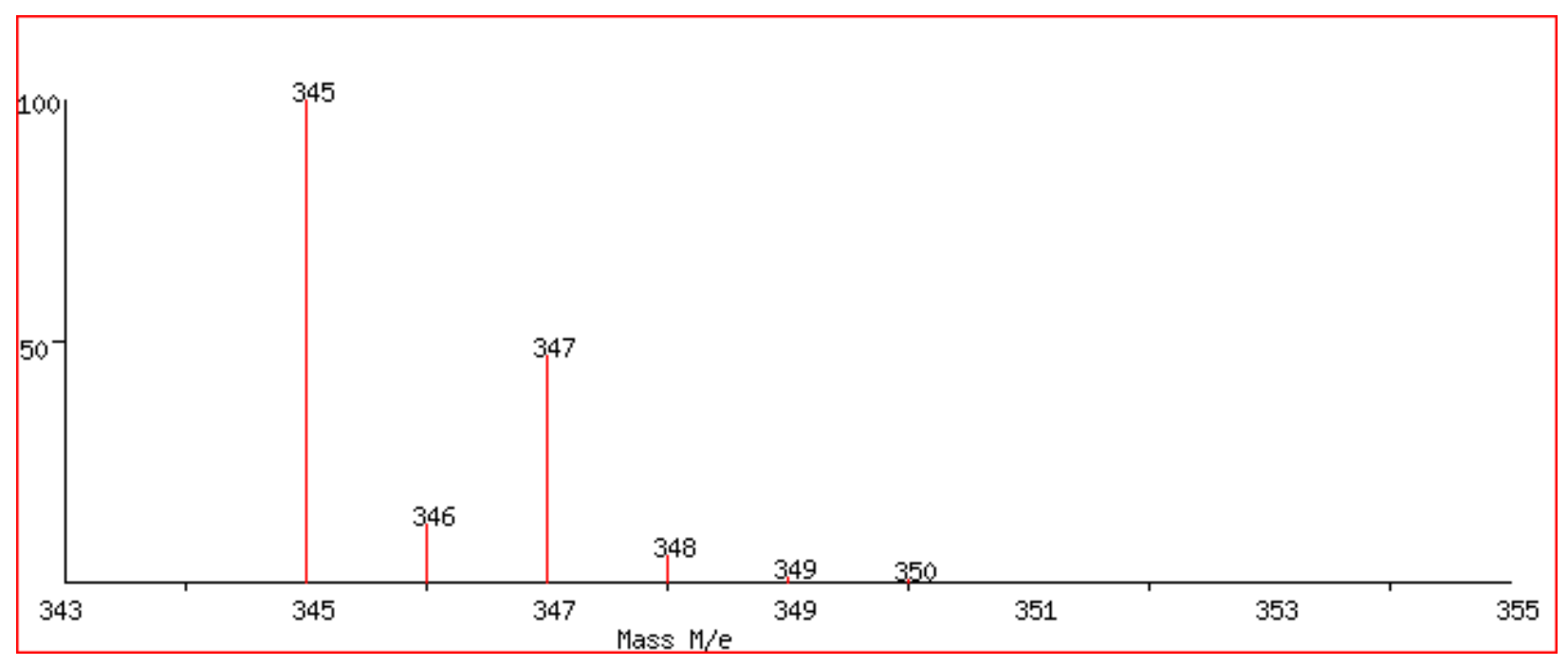

D. $\mathrm{C}_{10} \mathrm{H}_{20} \mathrm{O}_{5} \mathrm{CuNO}_{3}$

Figure C4.2 A-H Continued 


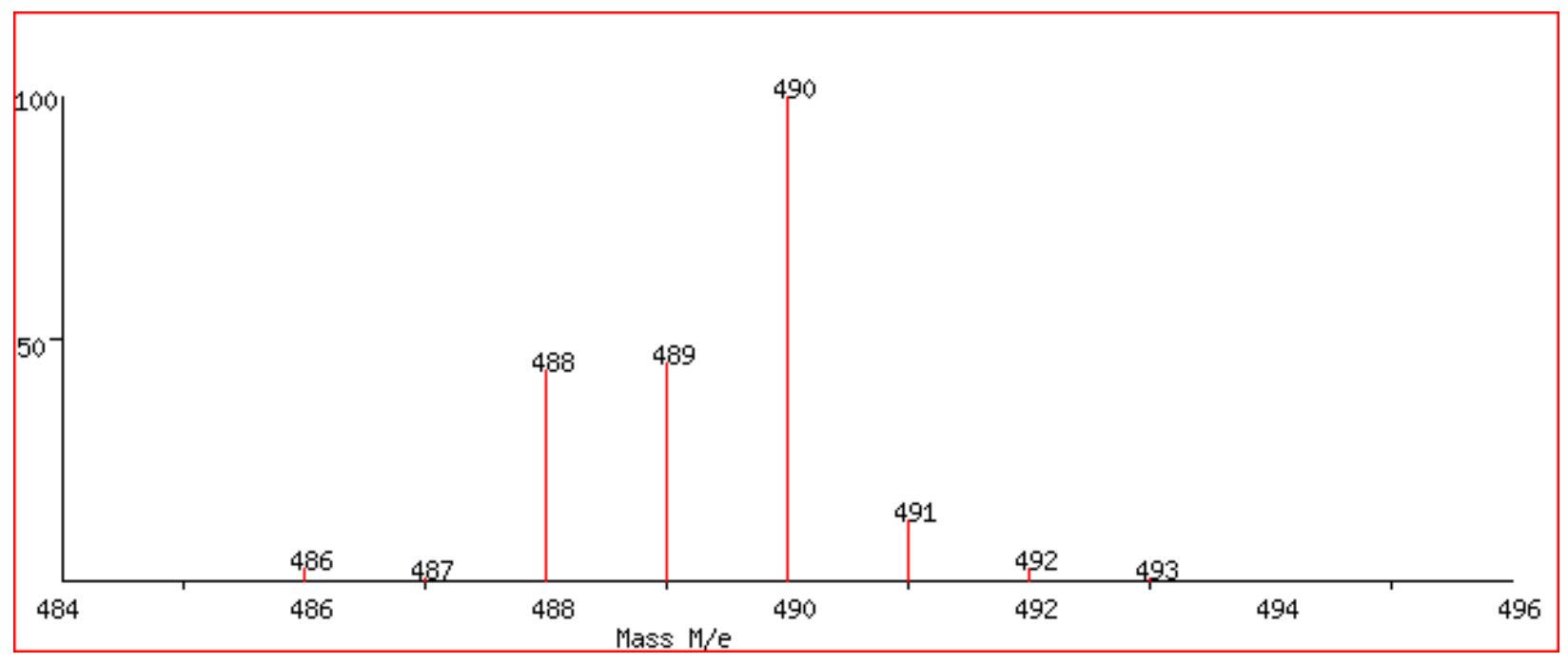

E. $\mathrm{C}_{10} \mathrm{H}_{20} \mathrm{O}_{5} \mathrm{PbNO}_{3}$

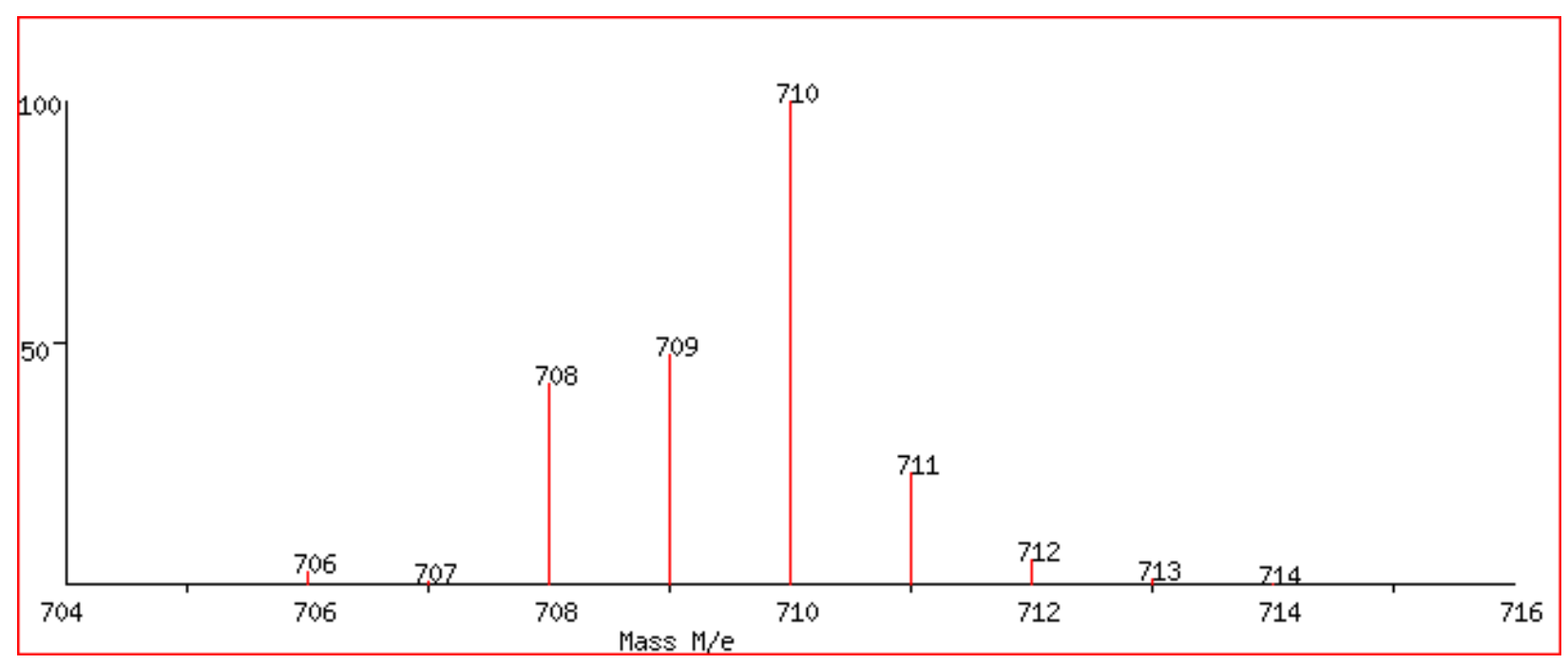

F. $\mathrm{C}_{20} \mathrm{H}_{40} \mathrm{O}_{10} \mathrm{PbNO}_{3}$

Figure C4.2 A-H Continued 


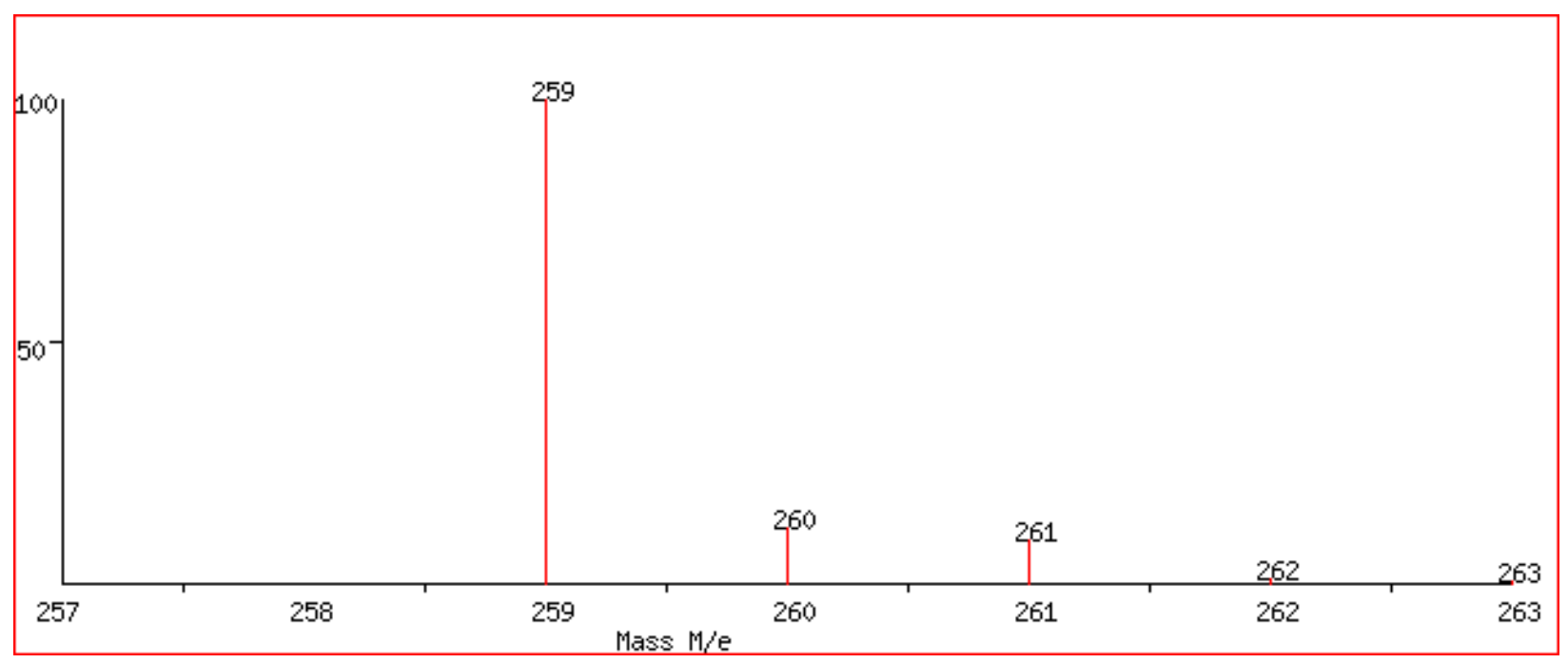

G. $\mathrm{C}_{10} \mathrm{H}_{20} \mathrm{O}_{5} \mathrm{~K}$

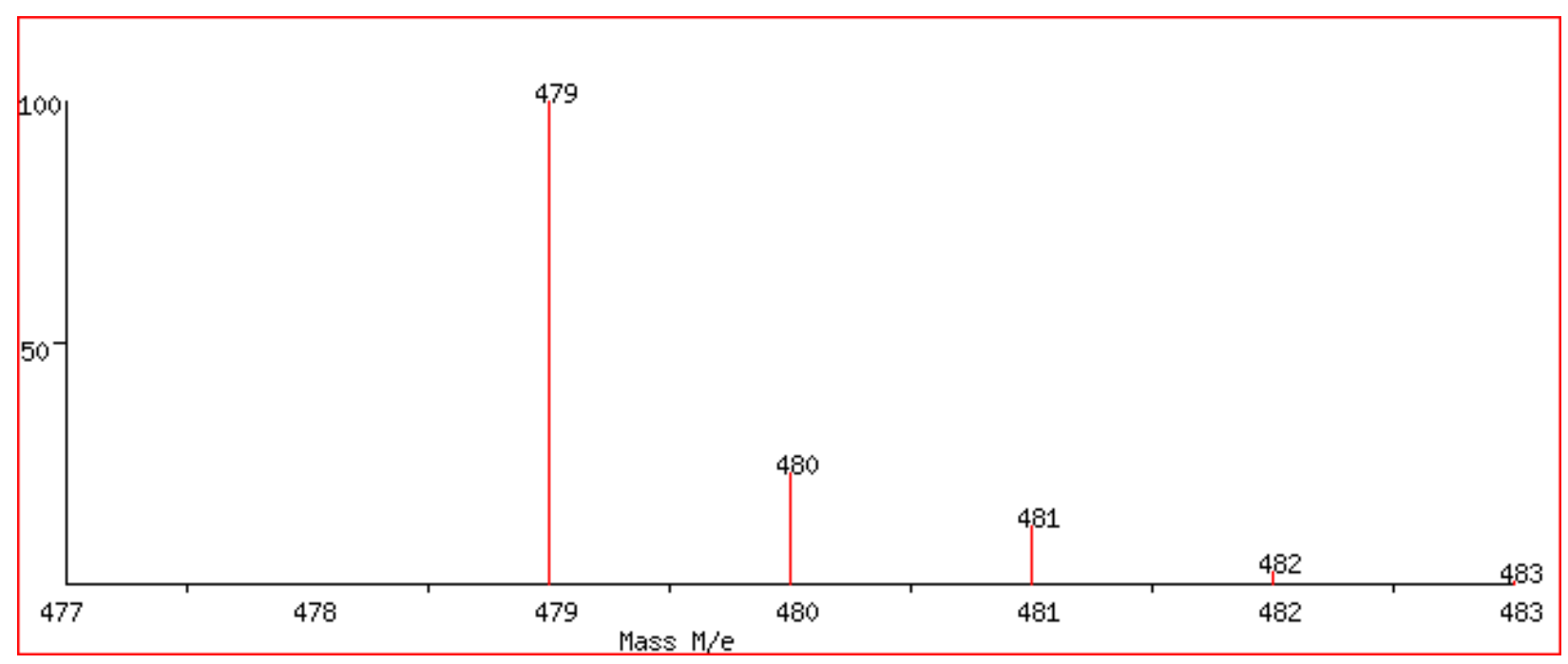

H. $\mathrm{C}_{20} \mathrm{H}_{40} \mathrm{O}_{10} \mathrm{~K}$

Figure C4.2 A-H Continued 


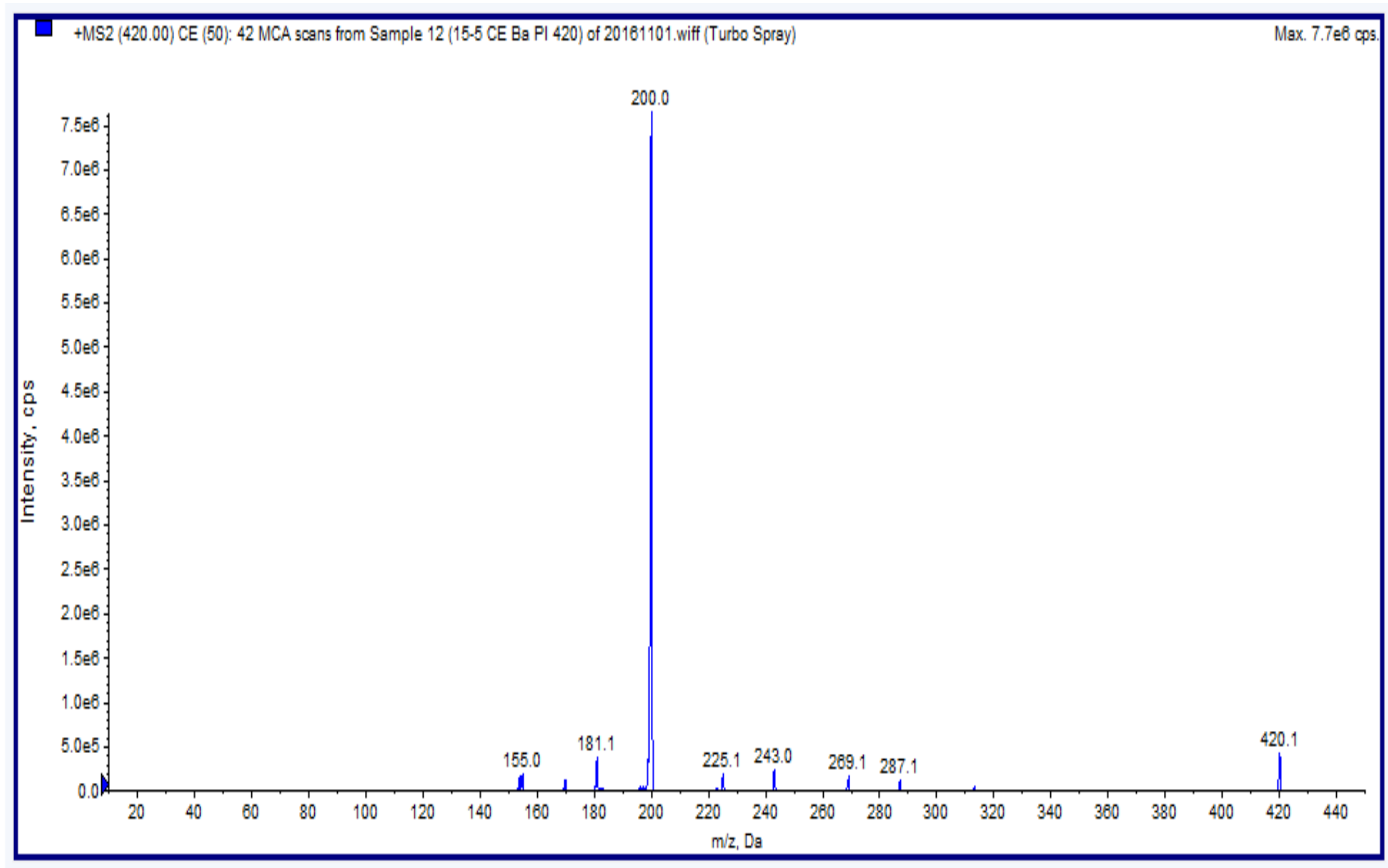

Figure C4.3 A-J Product ion spectra used for the identification of barium complex formation.

A. PI $m / z 420-\left[15-5+{ }^{138} \mathrm{Ba}+\mathrm{NO}_{3}\right]^{+}, m / z, 200-\left[{ }^{138} \mathrm{Ba}+\mathrm{NO}_{3}\right]^{+}$ 


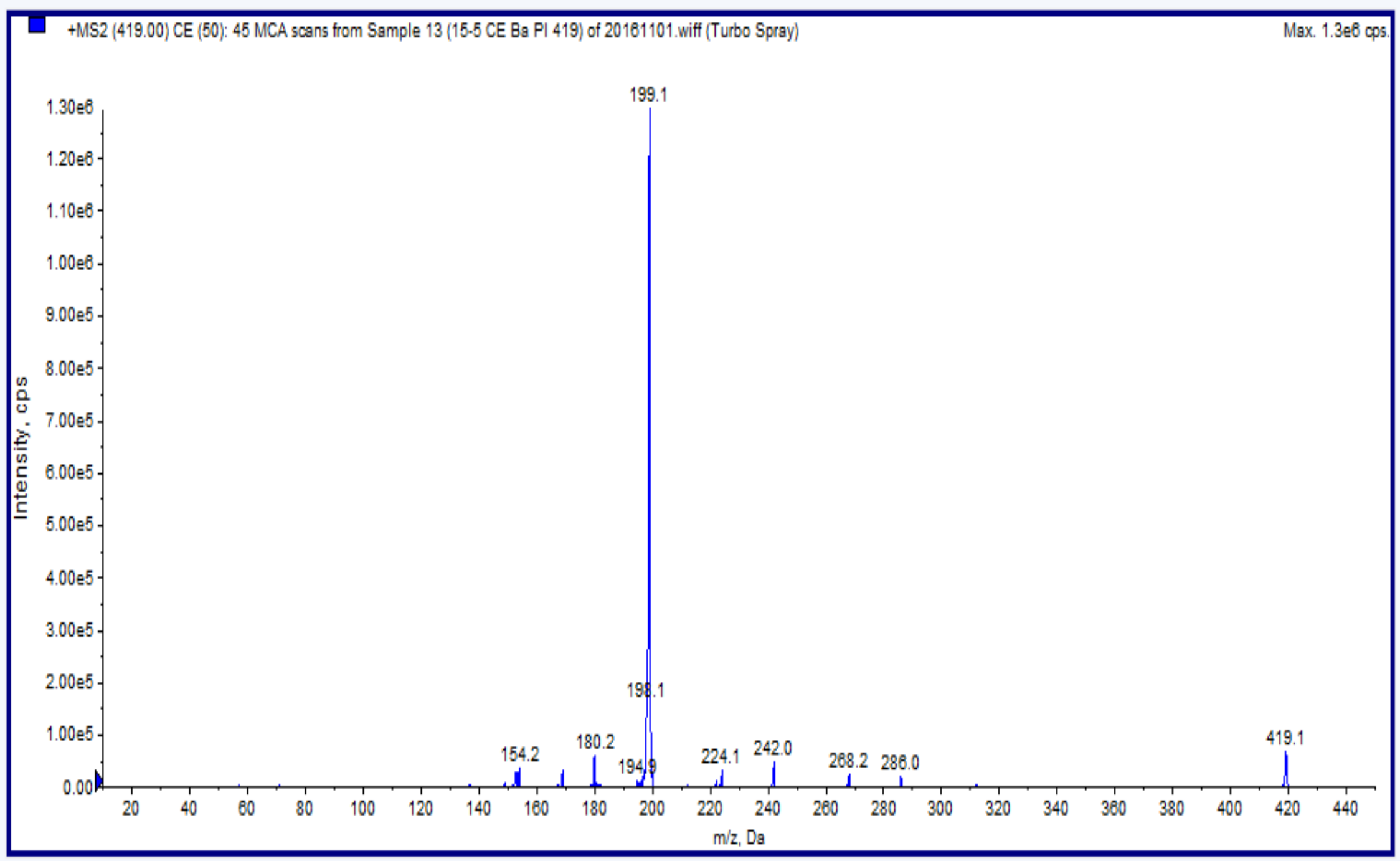

B. PI $m / z 419-\left[15-5+{ }^{137} \mathrm{Ba}+\mathrm{NO}_{3}\right]^{+}, m / z, 199-\left[{ }^{137} \mathrm{Ba}+\mathrm{NO}_{3}\right]^{+}$

Figure C4.3 A-J Continued 


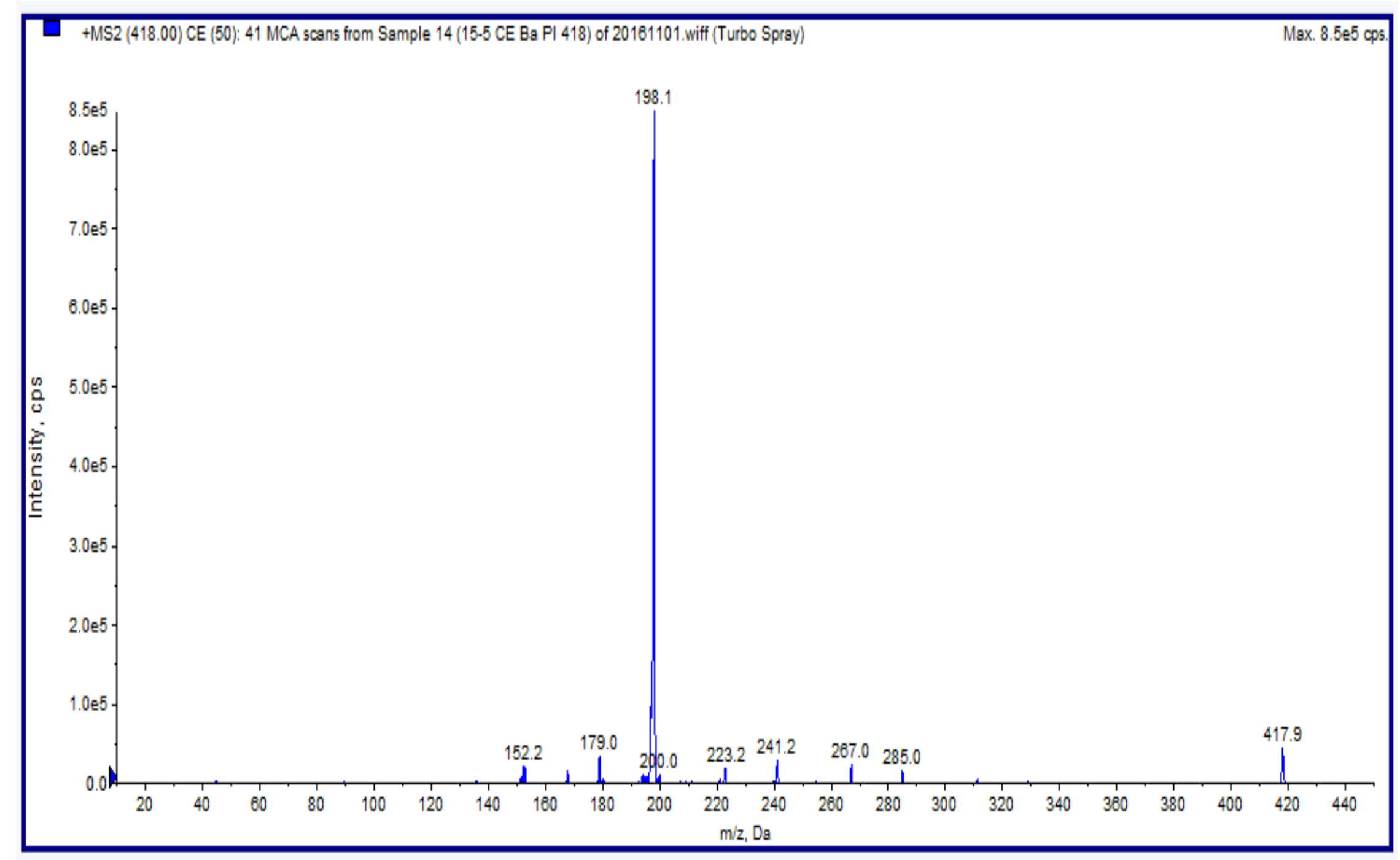

C. PI $m / z 418-\left[15-5+{ }^{136} \mathrm{Ba}+\mathrm{NO}_{3}\right]^{+}, m / z, 198-\left[{ }^{136} \mathrm{Ba}+\mathrm{NO}_{3}\right]^{+}$

Figure C4.3 A-J Continued 


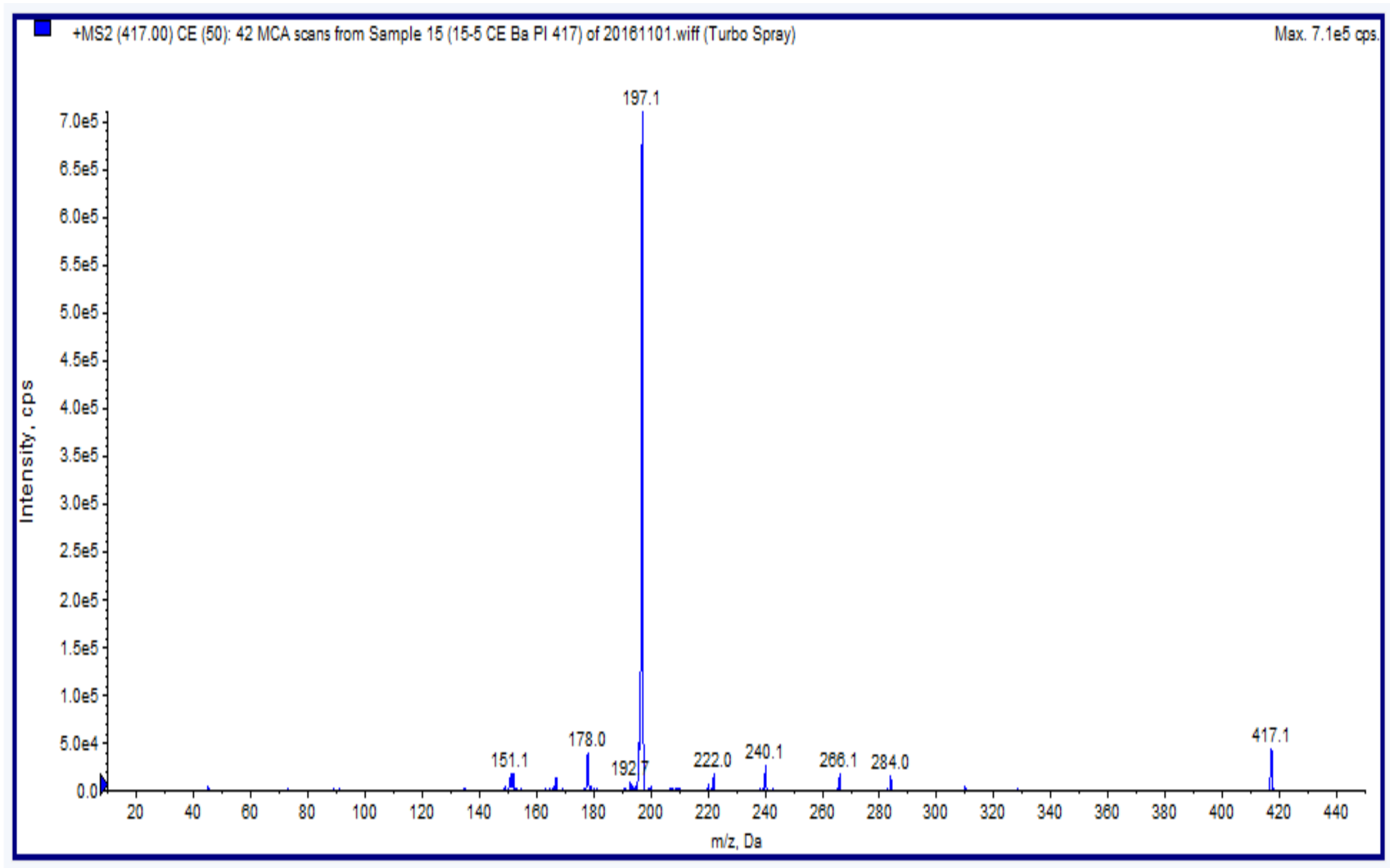

D. PI $m / z, 417-\left[15-5+{ }^{135} \mathrm{Ba}+\mathrm{NO}_{3}\right]^{+}, m / z, 197-\left[{ }^{135} \mathrm{Ba}+\mathrm{NO}_{3}\right]^{+}$

Figure C4.3 A-J Continued 


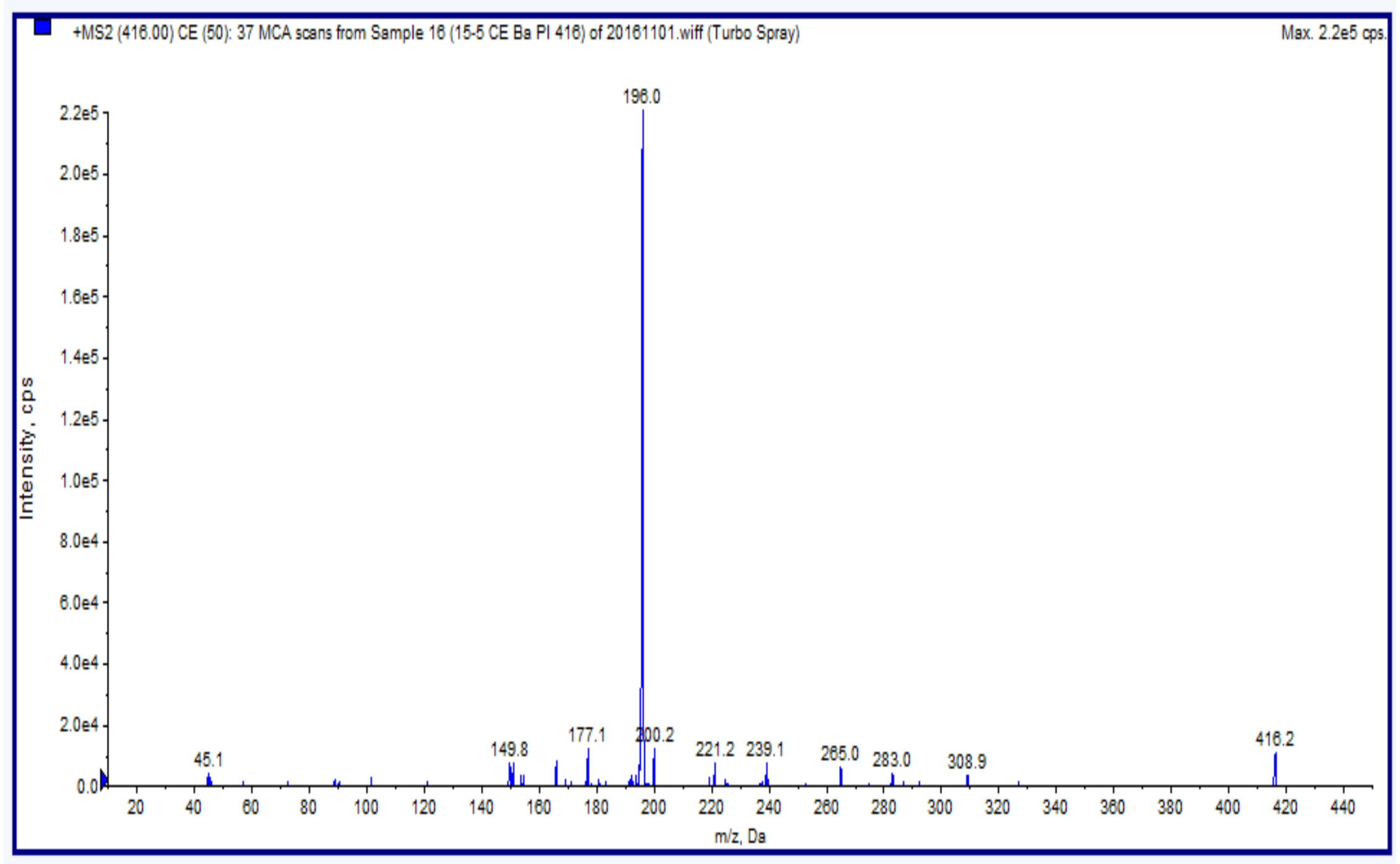

E. PI $m / z 416-\left[15-5+{ }^{134} \mathrm{Ba}+\mathrm{NO}_{3}\right]^{+}, m / z 194-\left[{ }^{134} \mathrm{Ba}+\mathrm{NO}_{3}\right]^{+}$

Figure C4.3 A-J Continued 


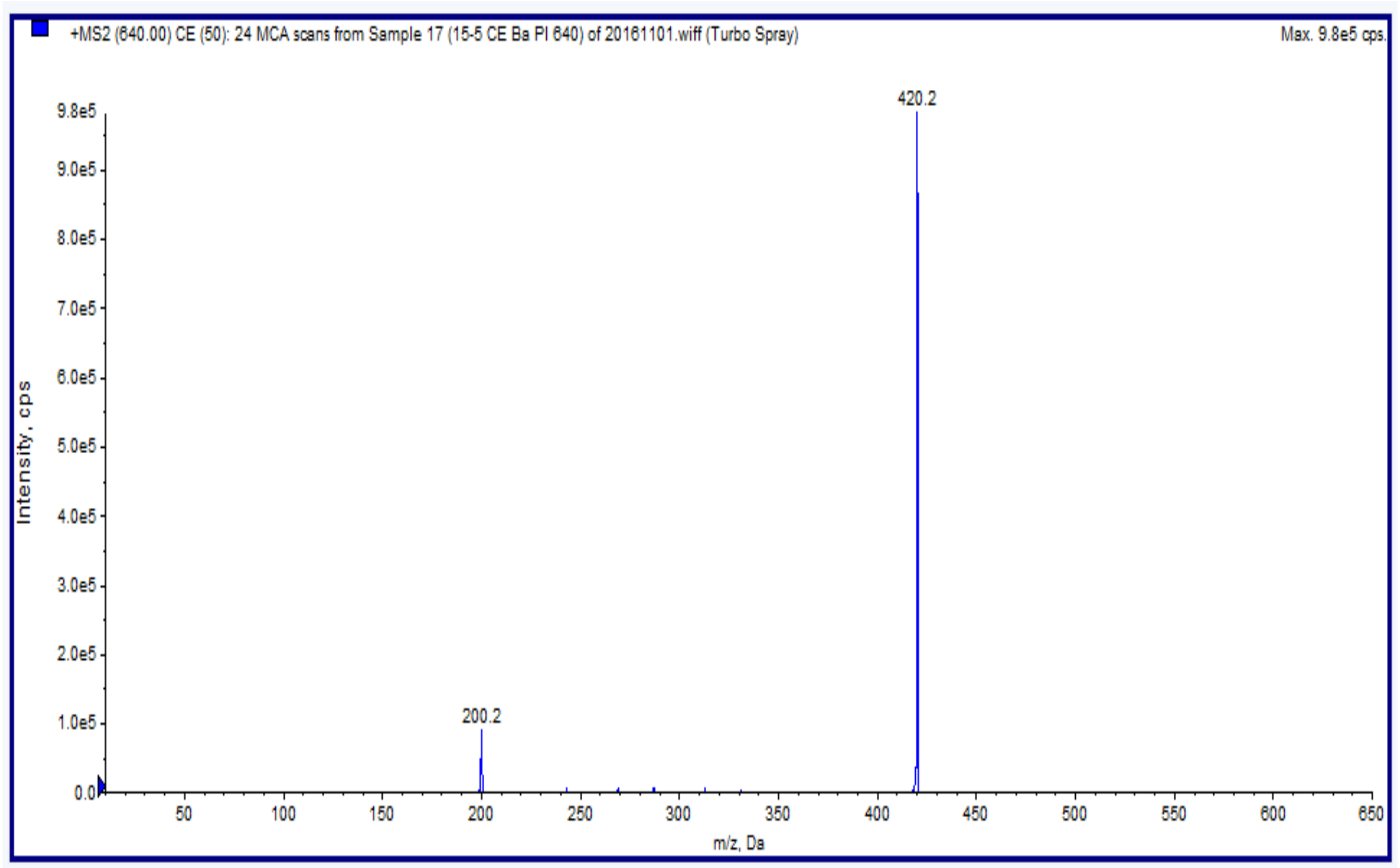

F. PI $m / z 640-\left[15-5_{2}+{ }^{138} \mathrm{Ba}+\mathrm{NO}_{3}\right]^{+}, m / z 420-\left[15-5+{ }^{138} \mathrm{Ba}+\mathrm{NO}_{3}\right]^{+}, m / z, 200-\left[{ }^{138} \mathrm{Ba}+\mathrm{NO}_{3}\right]^{+}$

Figure C4.3 A-J Continued 


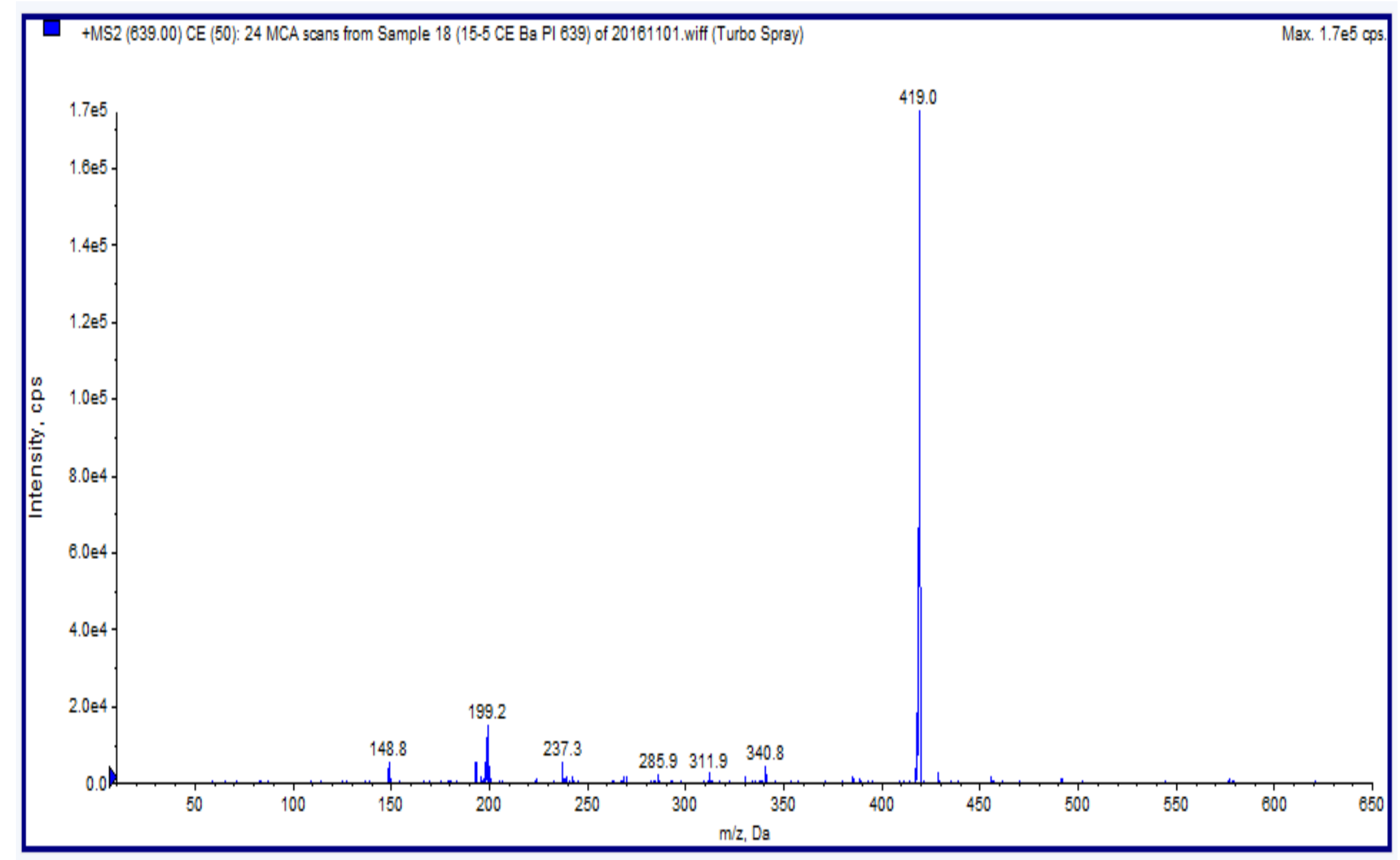

G. PI $m / z 639-\left[15-5_{2}+{ }^{137} \mathrm{Ba}+\mathrm{NO}_{3}\right]^{+}, m / z 419-\left[15-5+{ }^{137} \mathrm{Ba}+\mathrm{NO}_{3}\right]^{+}, m / z 199-\left[{ }^{137} \mathrm{Ba}+\mathrm{NO}_{3}\right]^{+}$

Figure C4.3 A-J Continued 


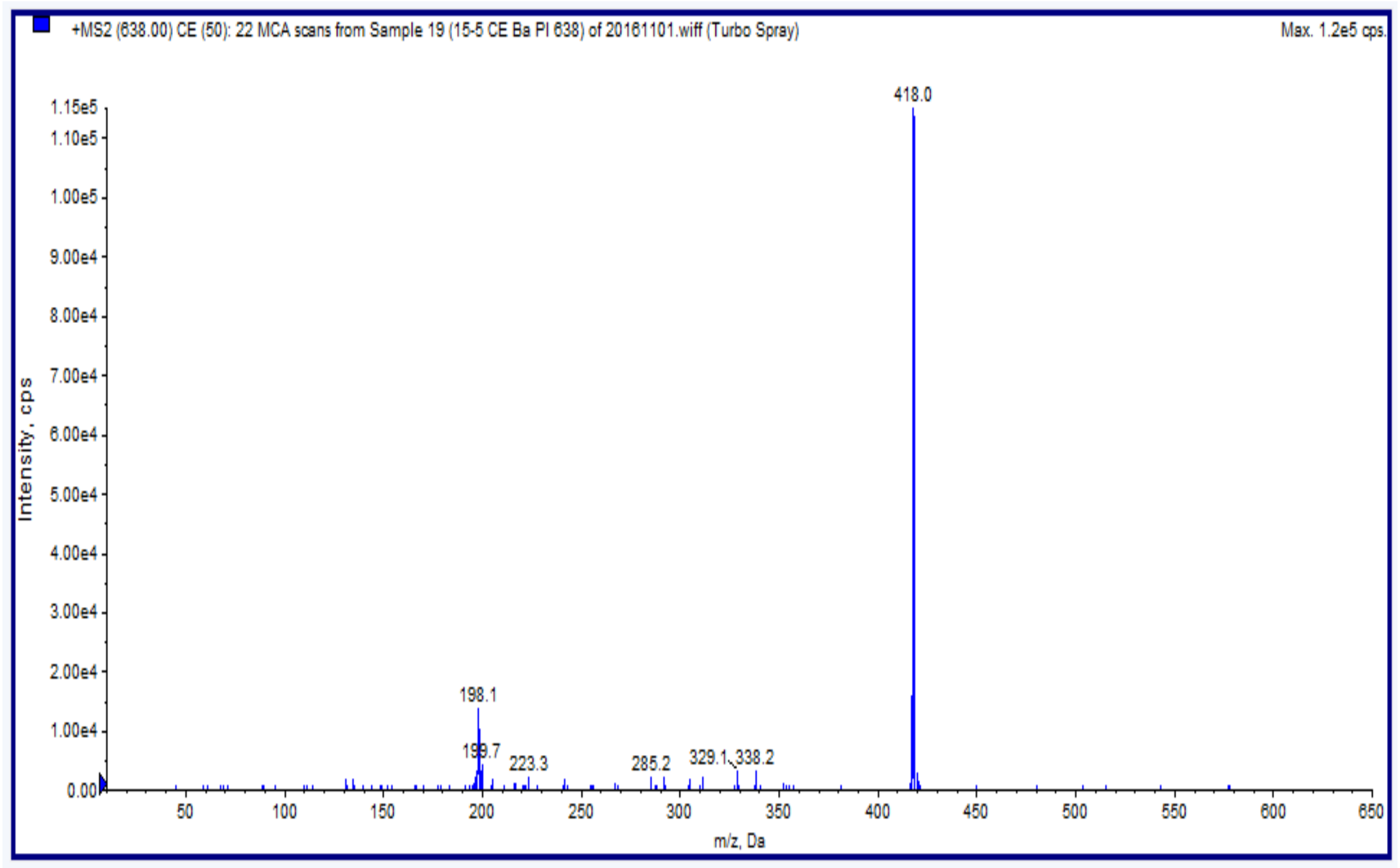

H. PI $m / z 638-\left[15-5_{2}+{ }^{136} \mathrm{Ba}+\mathrm{NO}_{3}\right]^{+}, m / z 418-\left[15-5+{ }^{136} \mathrm{Ba}+\mathrm{NO}_{3}\right]^{+}, m / z 198-\left[{ }^{136} \mathrm{Ba}+\mathrm{NO}_{3}\right]^{+}$

Figure C4.3 A-J Continued 


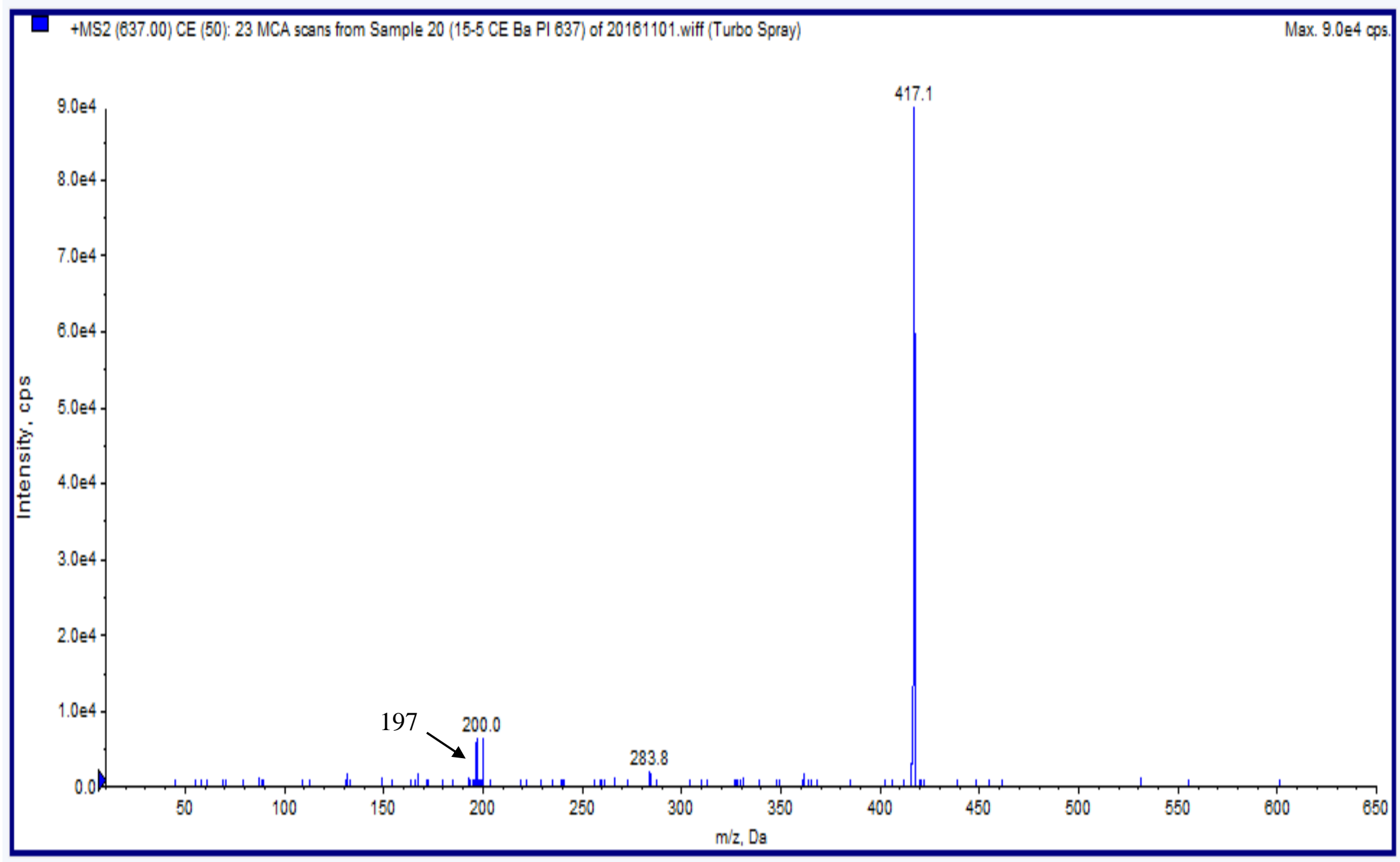

I. PI $m / z 637-\left[15-5_{2}+{ }^{135} \mathrm{Ba}+\mathrm{NO}_{3}\right]^{+}, m / z 417-\left[15-5+{ }^{135} \mathrm{Ba}+\mathrm{NO}_{3}\right]^{+}, m / z 197-\left[{ }^{135} \mathrm{Ba}+\mathrm{NO}_{3}\right]^{+}$

Figure C4.3 A-J Continued 


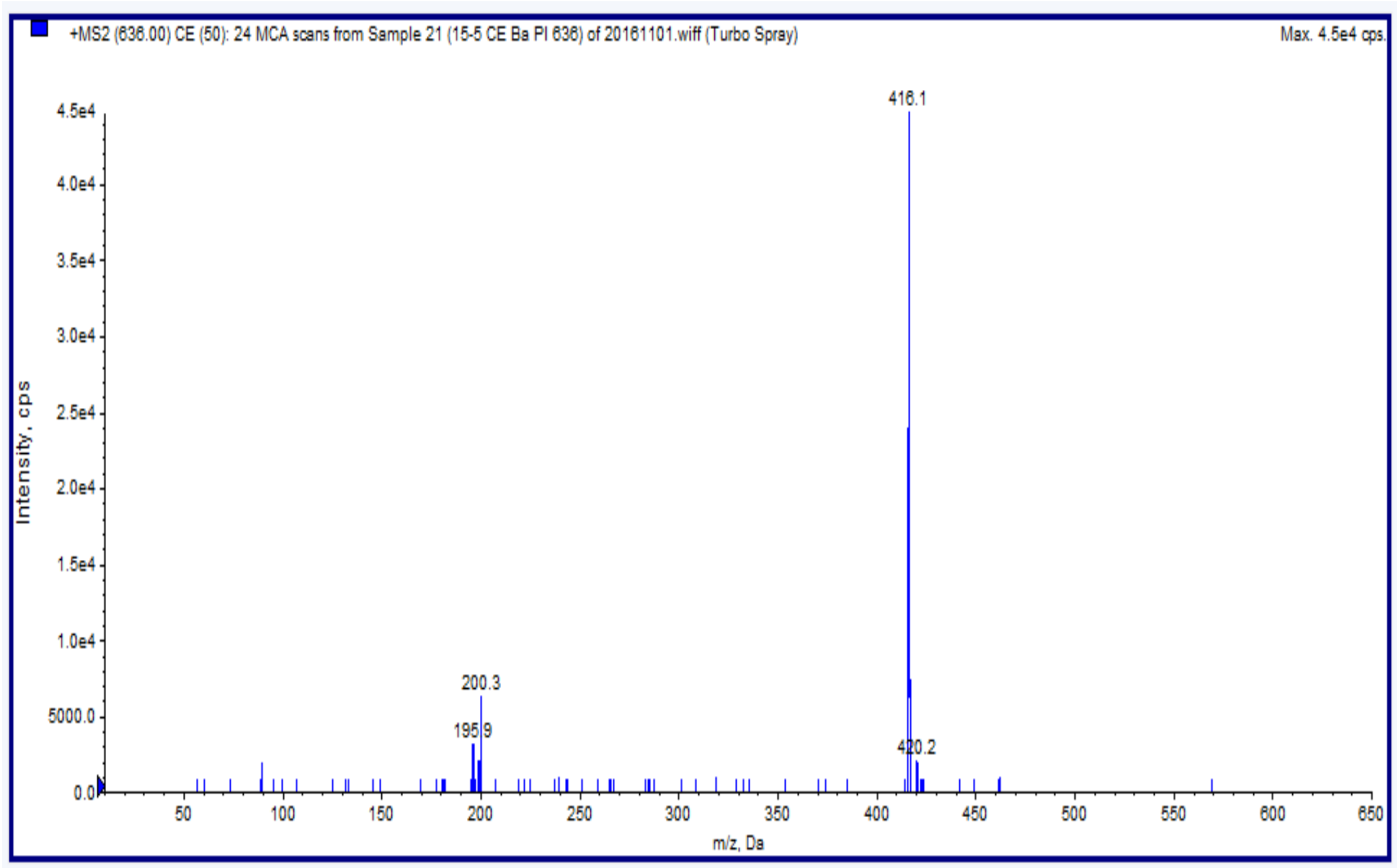

J. PI $m / z 636-\left[15-5_{2}+{ }^{134} \mathrm{Ba}+\mathrm{NO}_{3}\right]^{+}, m / z 416-\left[15-5+{ }^{134} \mathrm{Ba}+\mathrm{NO}_{3}\right]^{+}, m / z, 196-\left[{ }^{134} \mathrm{Ba}+\mathrm{NO}_{3}\right]^{+}$

Figure C4.3 A-J Continued 


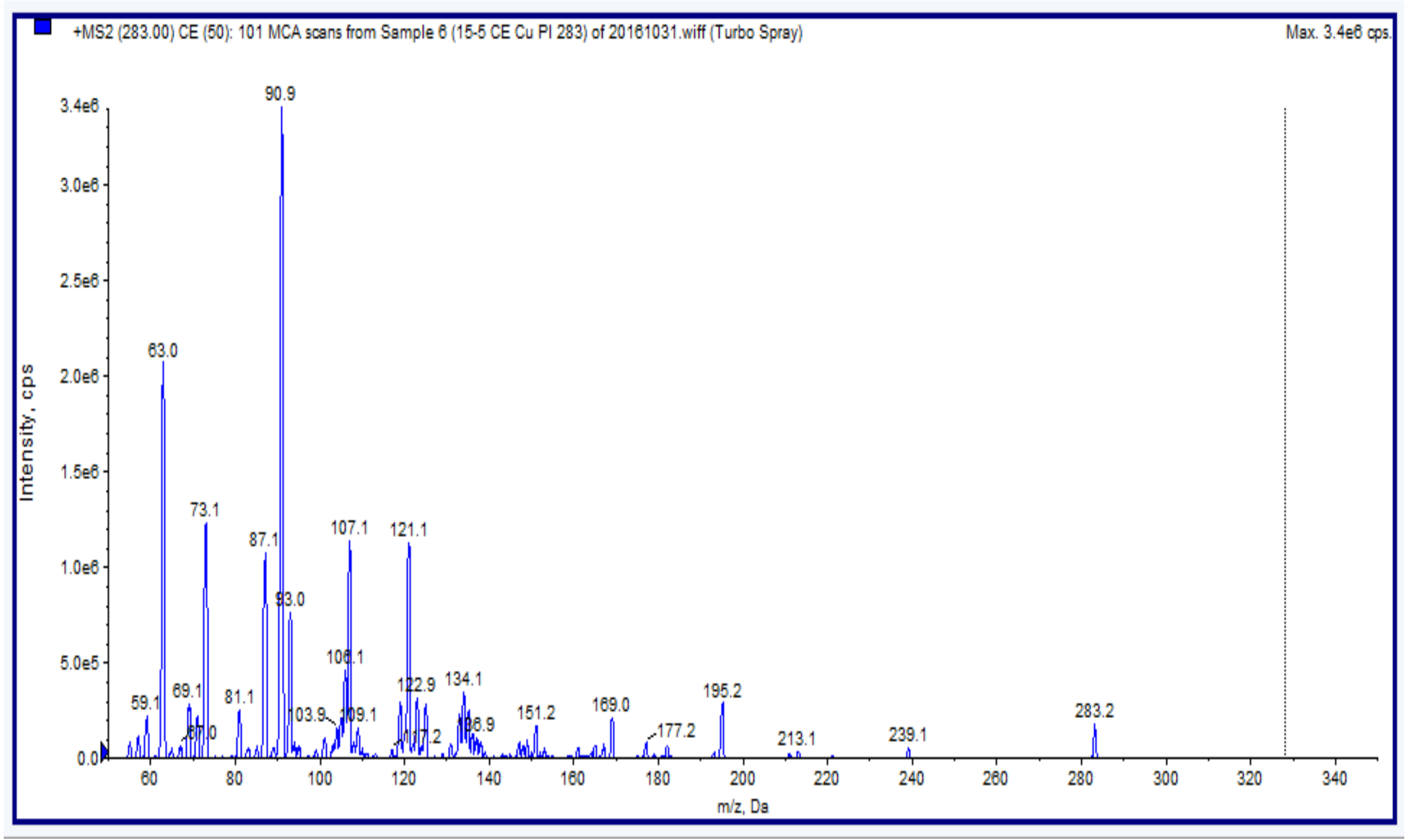

Figure C4.4A-D Product ion spectra used for the identification of copper complex formation.

A. PI $m / z 283-\left[15-5+{ }^{63} \mathrm{Cu}\right]^{+}, m / z, 63-\left[{ }^{63} \mathrm{Cu}\right]^{+}$ 


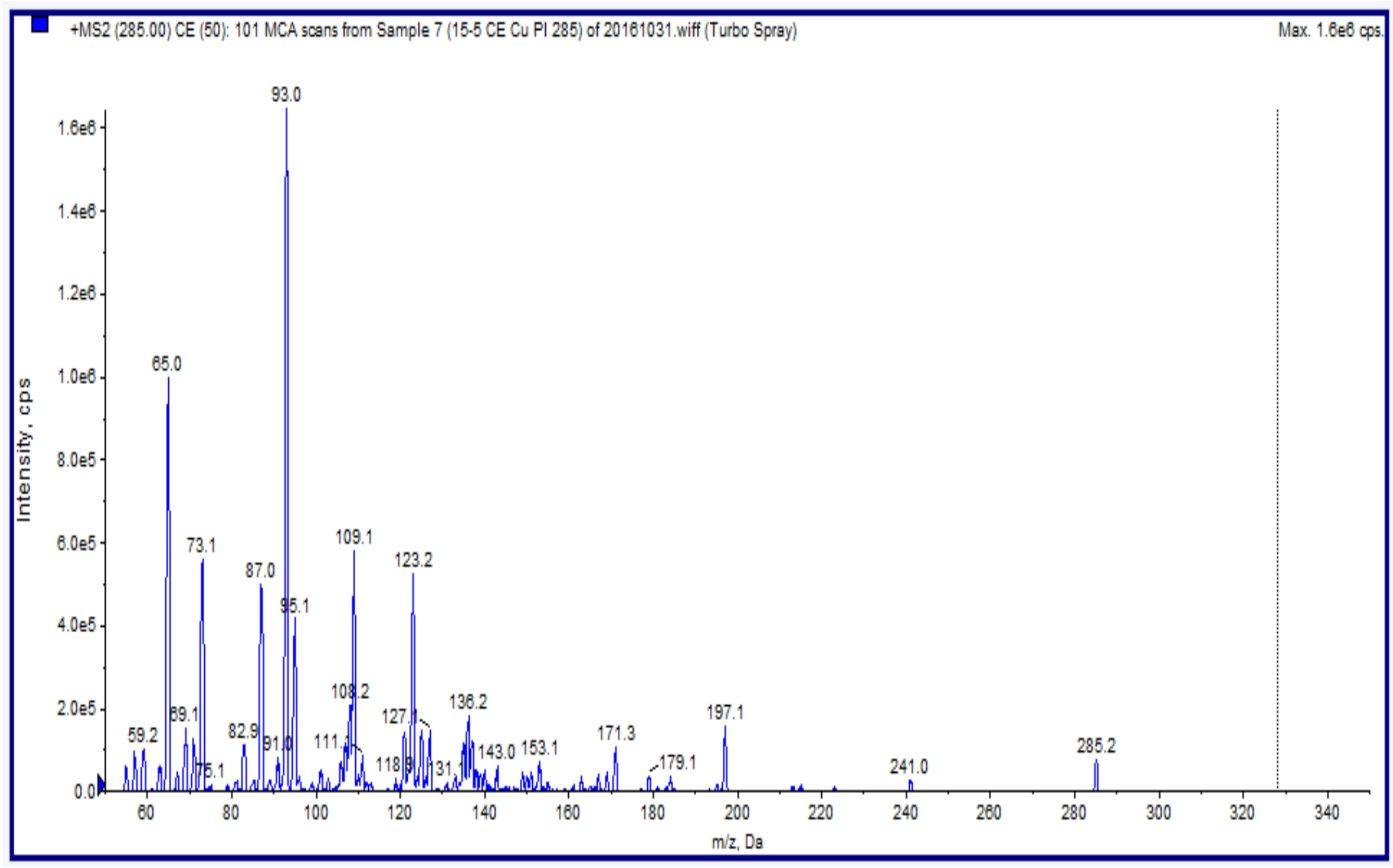

B. PI $m / z 285-\left[15-5+{ }^{65} \mathrm{Cu}\right]^{+}, m / z 65-\left[{ }^{65} \mathrm{Cu}\right]^{+}$

Figure C4.4 A-D Continued 


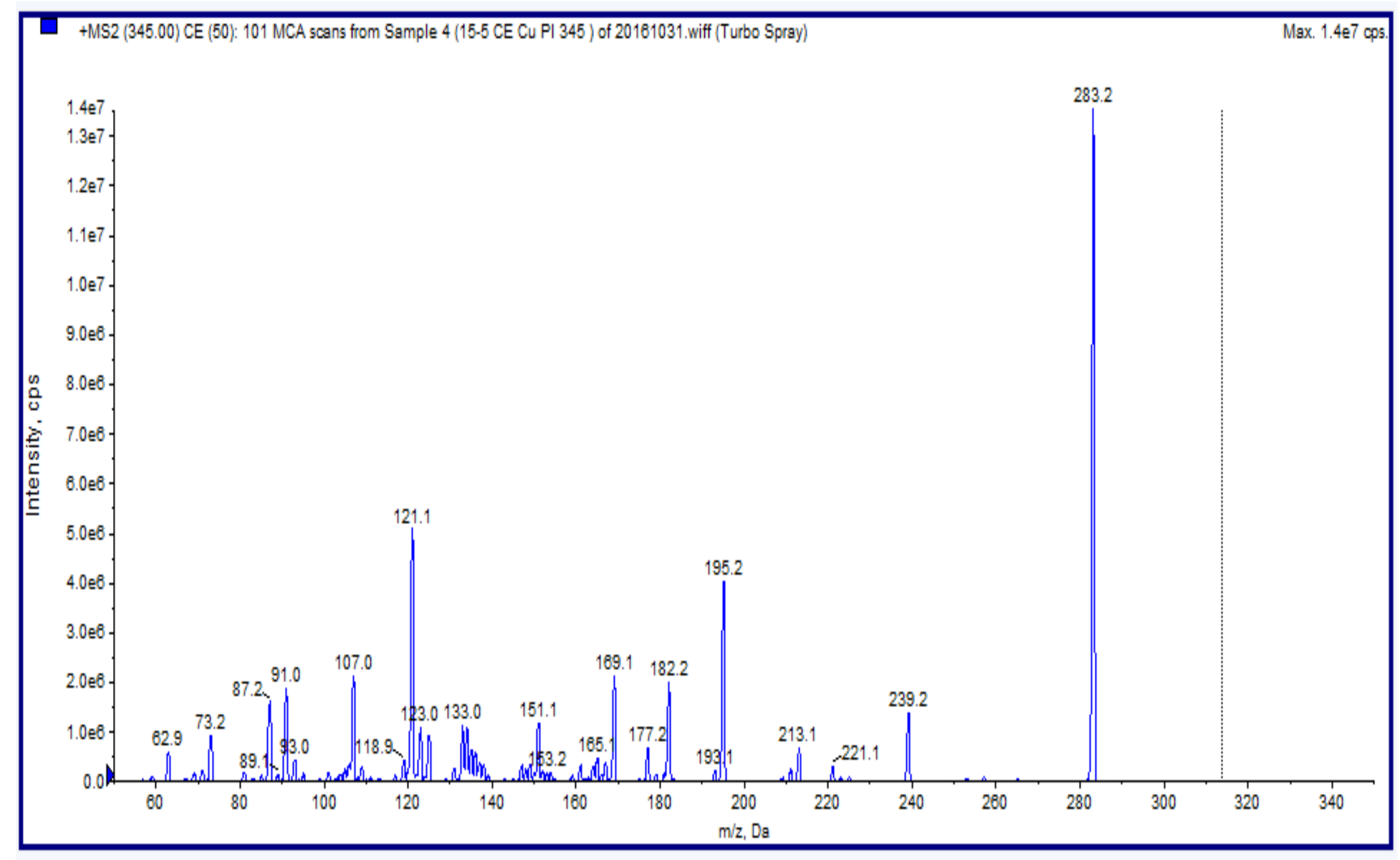

C. PI $m / z 345-\left[15-5+{ }^{63} \mathrm{Cu}+\mathrm{NO}_{3}\right]^{+}, m / z 283-\left[15-5+{ }^{63} \mathrm{Cu}\right]^{+}, m / z 63-\left[{ }^{63} \mathrm{Cu}\right]^{+}$

Figure C4.4 A-D Continued 


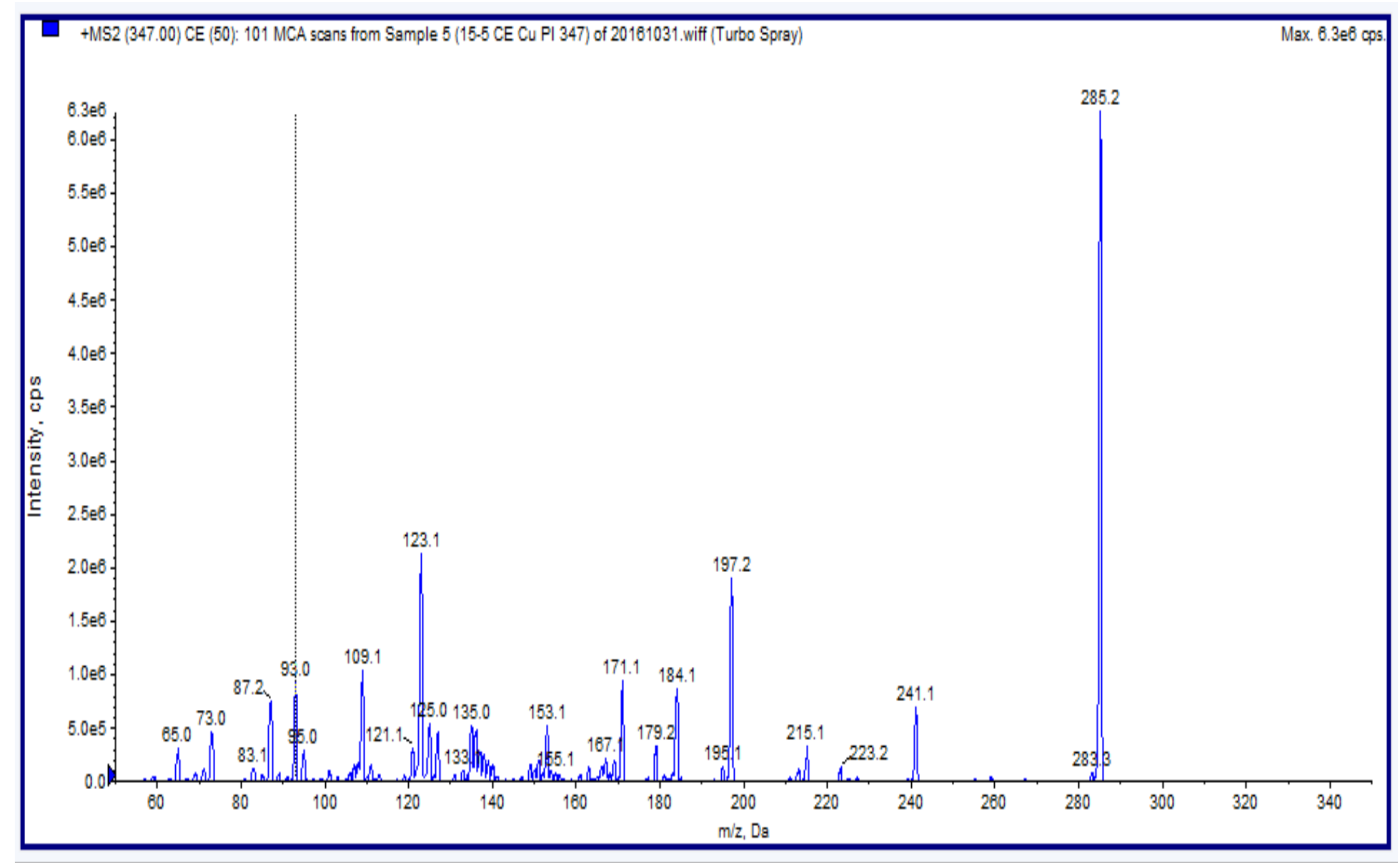

D. PI $m / z 347-\left[15-5+{ }^{65} \mathrm{Cu}+\mathrm{NO}_{3}\right]^{+}, m / z 285-\left[15-5+{ }^{65} \mathrm{Cu}\right]^{+}, m / z 65-\left[{ }^{65} \mathrm{Cu}\right]^{+}$

Figure C4.4 A-D Continued 


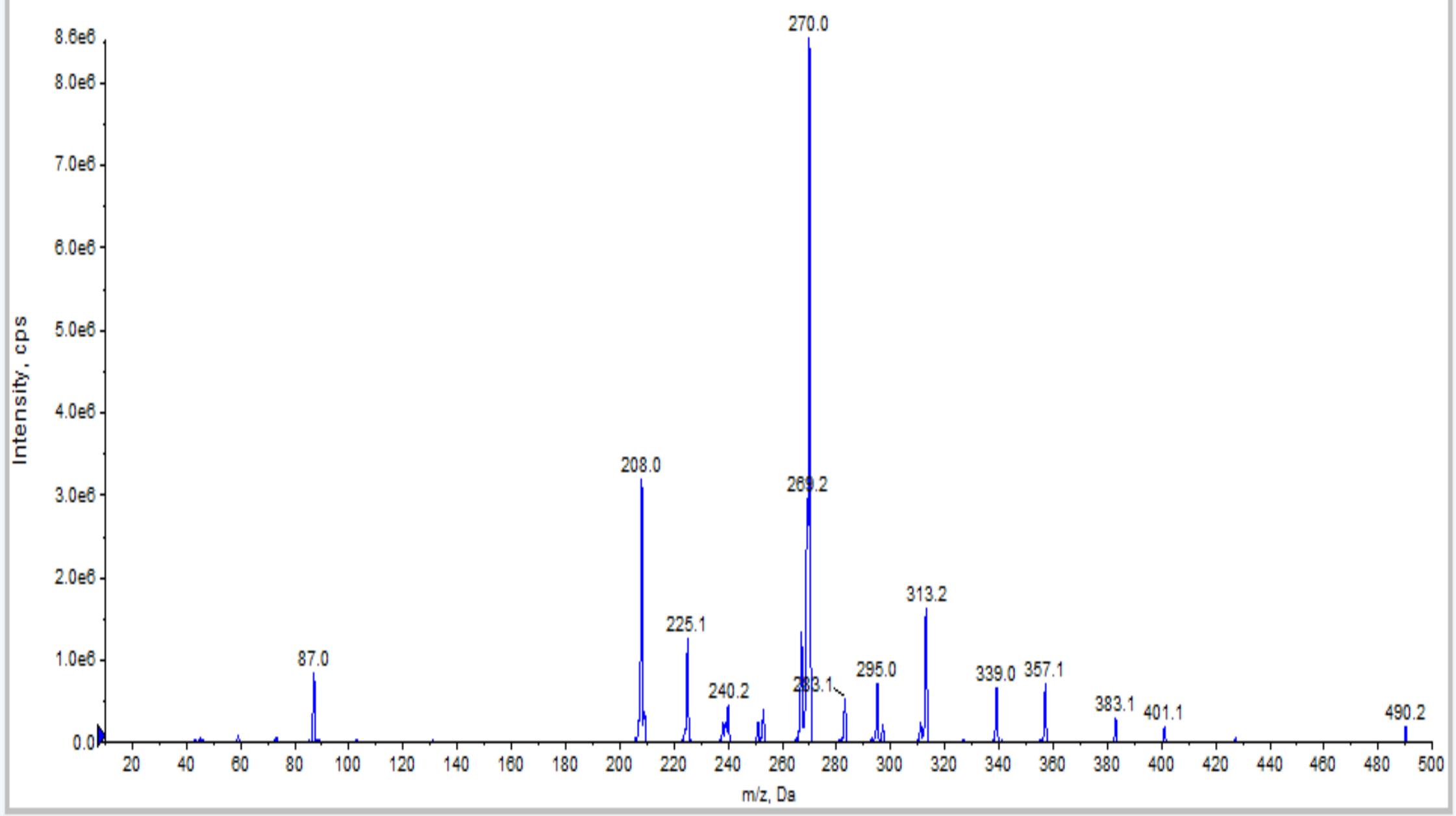

Figure C4.5 A-H Product ion spectra used for the identification of lead complex formation.

A. PI $m / z 490-\left[15-5+{ }^{208} \mathrm{~Pb}+\mathrm{NO}_{3}\right]^{+}, m / z 270-\left[{ }^{208} \mathrm{~Pb}+\mathrm{NO}_{3}\right]^{+}, m / z 208-\left[{ }^{208} \mathrm{~Pb}\right]^{+}$ 


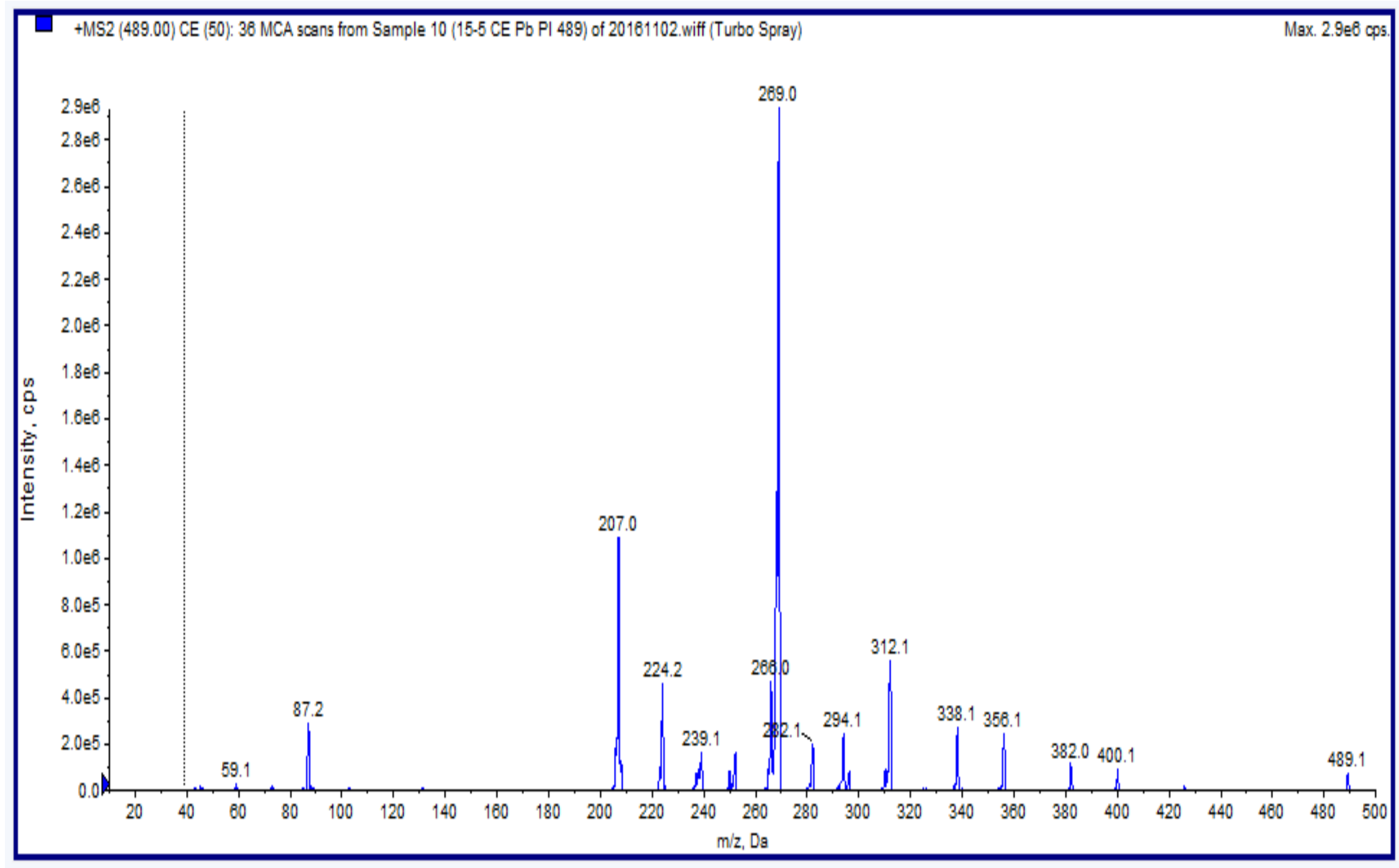

B. PI $m / z 489-\left[15-5+{ }^{207} \mathrm{~Pb}+\mathrm{NO}_{3}\right]^{+}, m / z 269-\left[{ }^{207} \mathrm{~Pb}+\mathrm{NO}_{3}\right]^{+}, m / z, 207-\left[{ }^{207} \mathrm{~Pb}\right]^{+}$

Figure C4.5 A-H Continued 


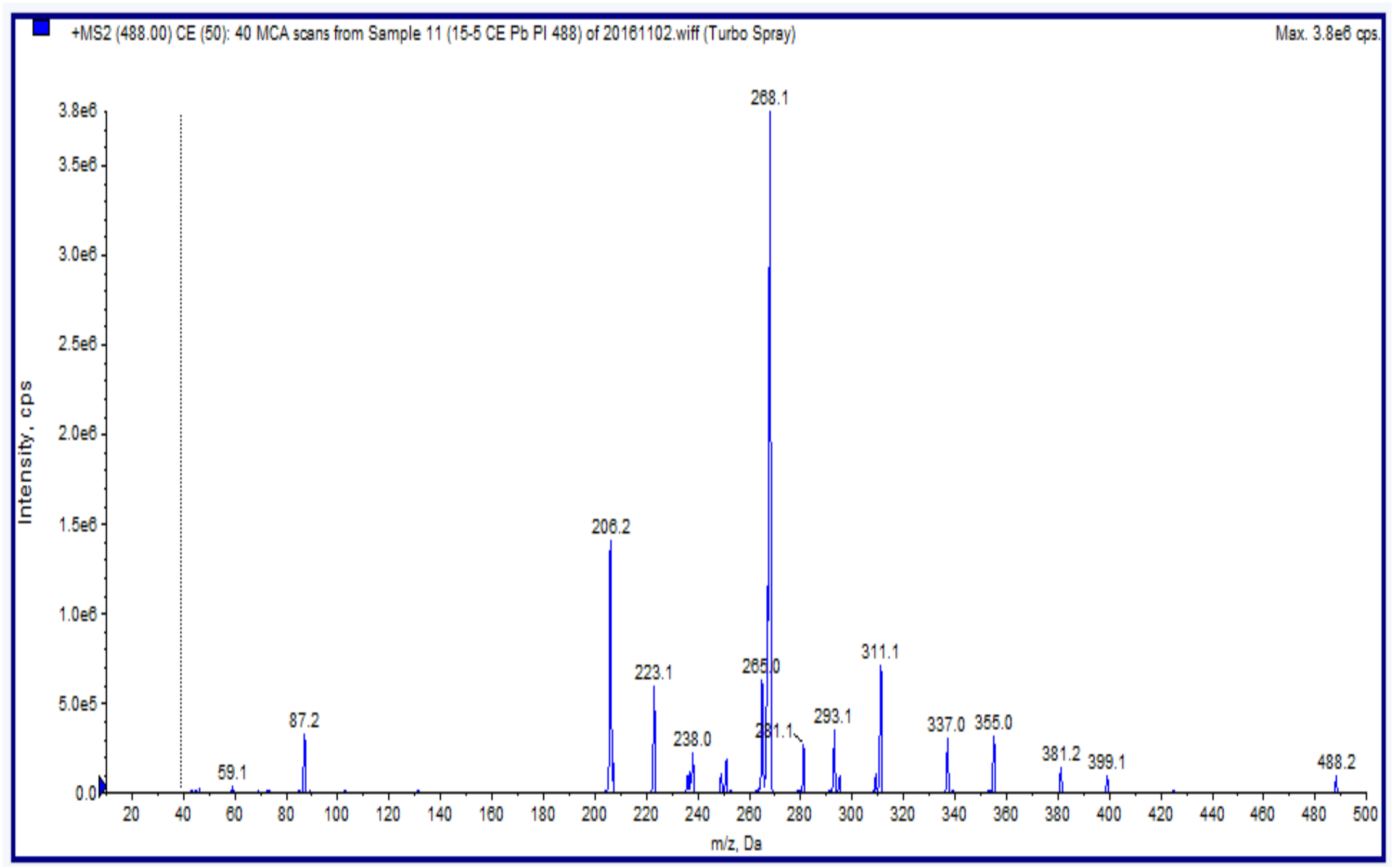

C. PI $m / z 488-\left[15-5+{ }^{206} \mathrm{~Pb}+\mathrm{NO}_{3}\right]^{+}, m / z 268-\left[{ }^{206} \mathrm{~Pb}+\mathrm{NO}_{3}\right]^{+}, m / z 206-\left[{ }^{206} \mathrm{~Pb}\right]^{+}$

Figure C4.5 A-H Continued 


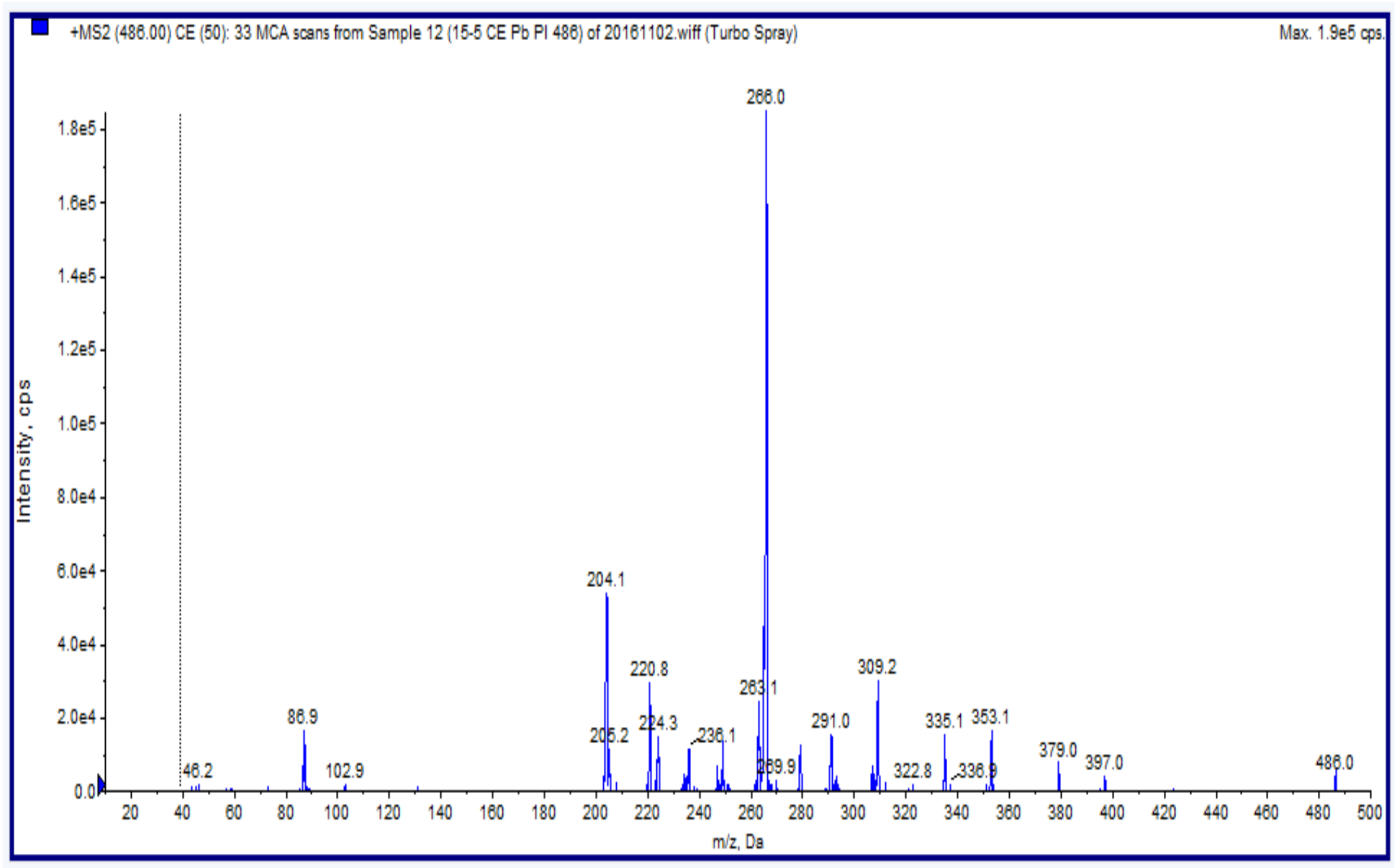

D. PI $m / z 486-\left[15-5+{ }^{204} \mathrm{~Pb}+\mathrm{NO}_{3}\right]^{+}, m / z 266-\left[{ }^{204} \mathrm{~Pb}+\mathrm{NO}_{3}\right]^{+}, m / z 204-\left[{ }^{204} \mathrm{~Pb}\right]^{+}$

Figure C4.5 A-H Continued 


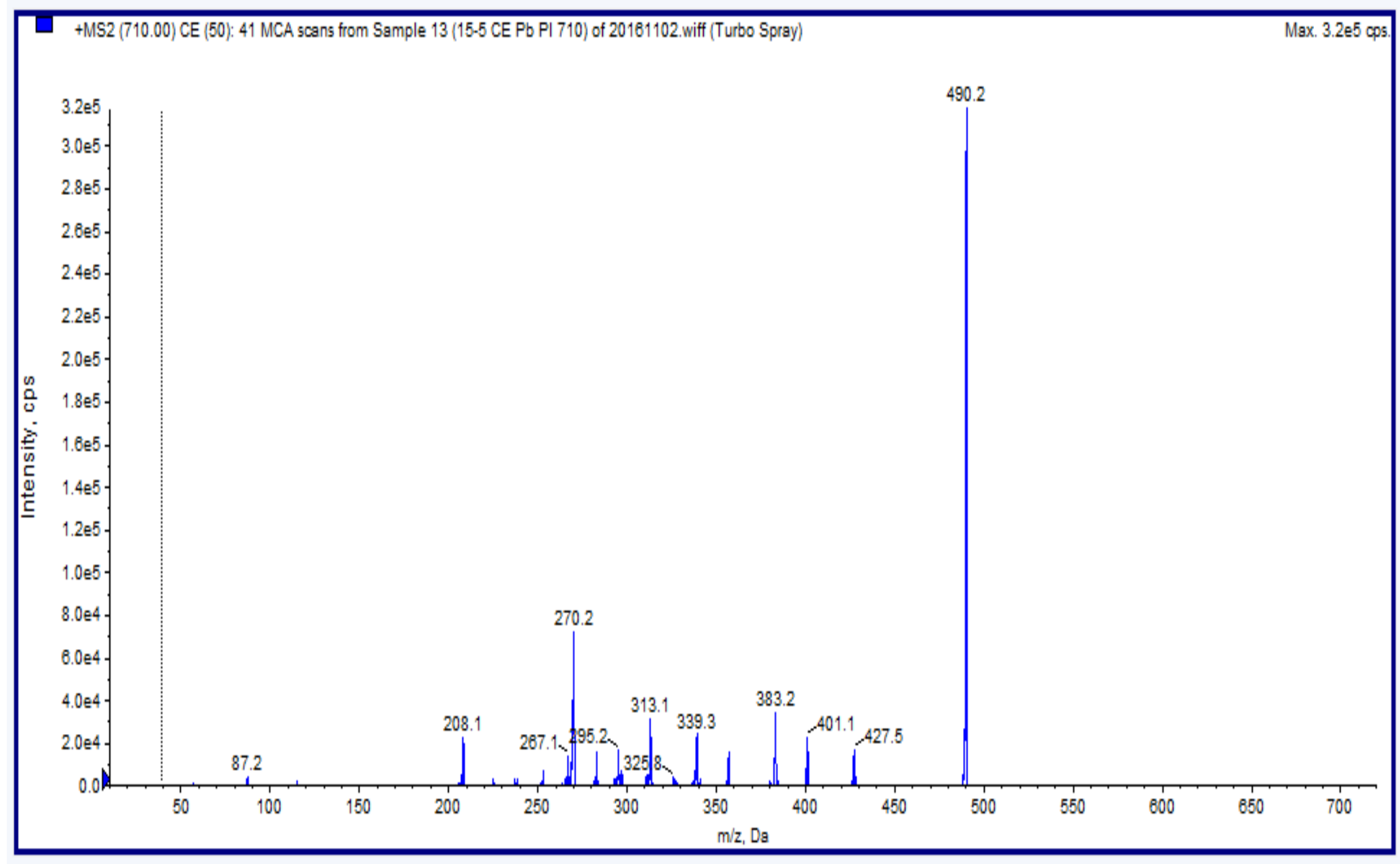

E. PI $m / z 710-\left[15-5_{2}+{ }^{208} \mathrm{~Pb}+\mathrm{NO}_{3}\right]^{+}, m / z 490-\left[15-5+{ }^{208} \mathrm{~Pb}+\mathrm{NO}_{3}\right]^{+}, m / z 270-\left[{ }^{208} \mathrm{~Pb}+\mathrm{NO}_{3}\right]^{+}, m / z 208-\left[{ }^{208} \mathrm{~Pb}\right]^{+}$

Figure C4.5 A-H Continued 


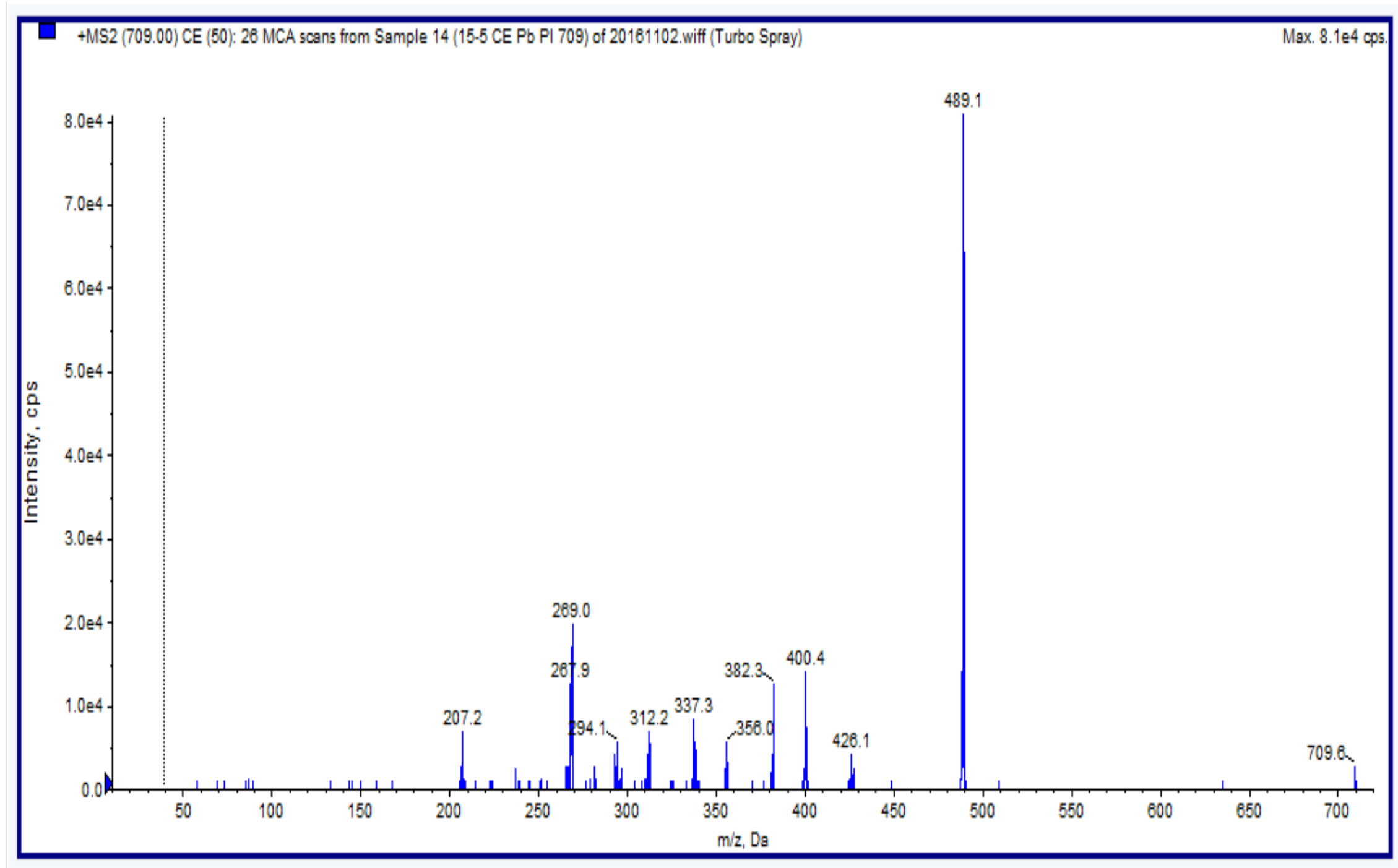

F. PI $m / z 709-\left[15-5_{2}+{ }^{207} \mathrm{~Pb}+\mathrm{NO}_{3}\right]^{+}, m / z 489-\left[15-5+{ }^{207} \mathrm{~Pb}+\mathrm{NO}_{3}\right]^{+}, m / z, 269-\left[{ }^{207} \mathrm{~Pb}+\mathrm{NO}_{3}\right]^{+}, m / z 207-\left[{ }^{207} \mathrm{~Pb}\right]^{+}$

Figure C4.5 A-H Continued 


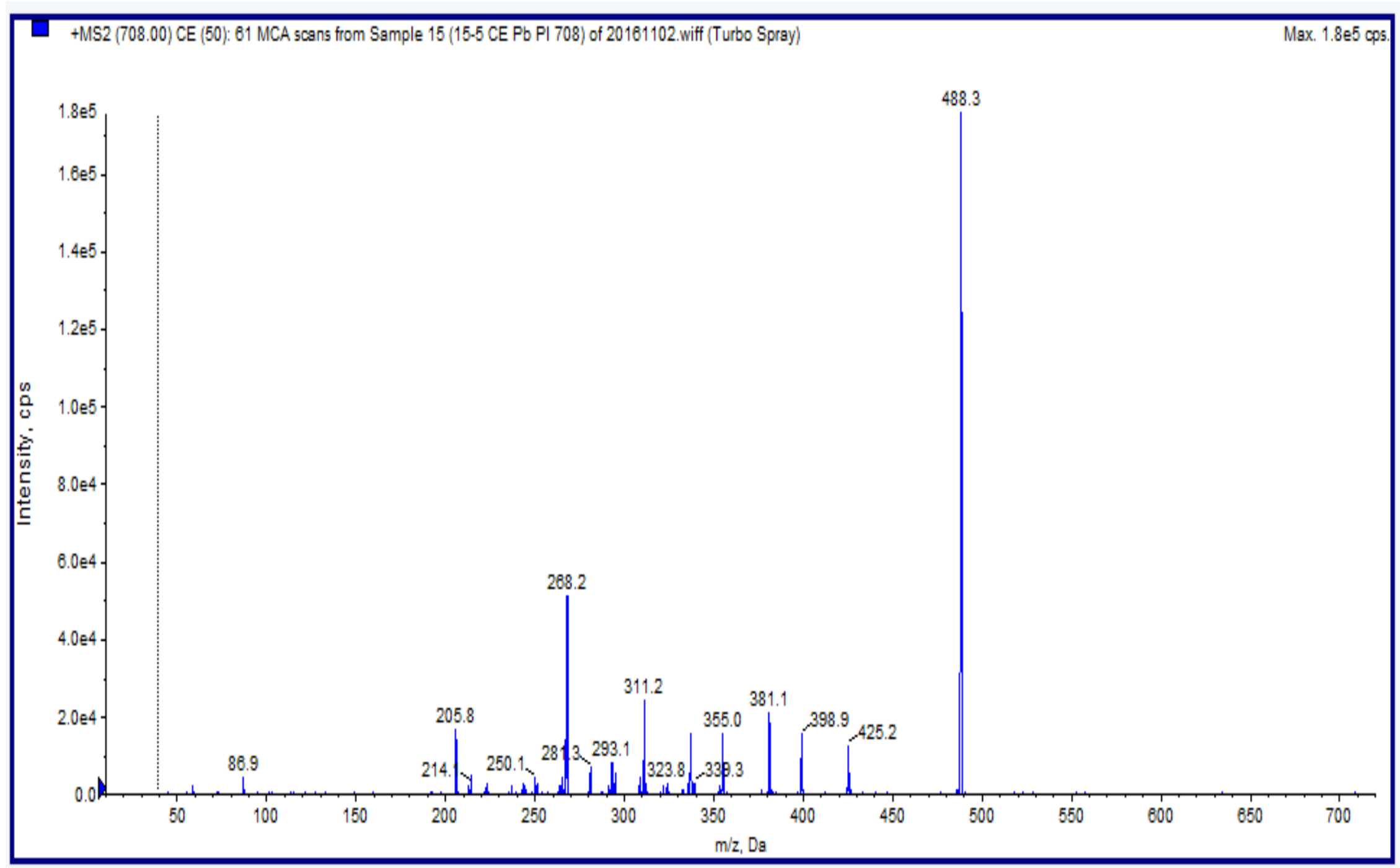

G. PI $m / z 708-\left[15-5_{2}+{ }^{206} \mathrm{~Pb}+\mathrm{NO}_{3}\right]^{+}, m / z 488-\left[15-5+{ }^{206} \mathrm{~Pb}+\mathrm{NO}_{3}\right]^{+}, m / z, 268-\left[{ }^{206} \mathrm{~Pb}+\mathrm{NO}_{3}\right]^{+}, m / z 206-\left[{ }^{206} \mathrm{~Pb}\right]^{+}$

Figure C4.5 A-H Continued 


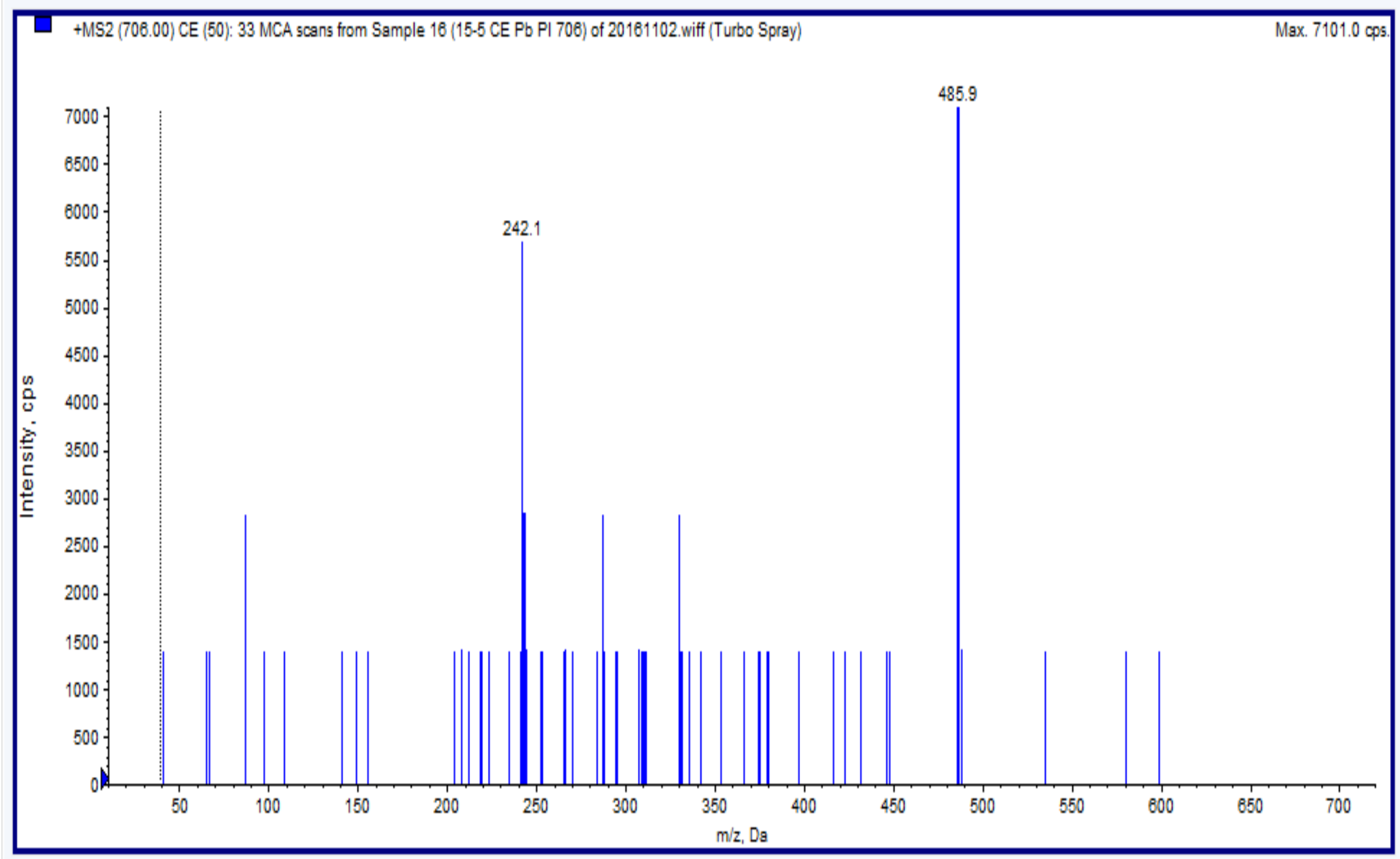

H. PI $m / z 706-\left[15-5_{2}+{ }^{204} \mathrm{~Pb}+\mathrm{NO}_{3}\right]^{+}, m / z 486-\left[15-5+{ }^{204} \mathrm{~Pb}+\mathrm{NO}_{3}\right]^{+}$

Figure C4.5 A-H Continued 


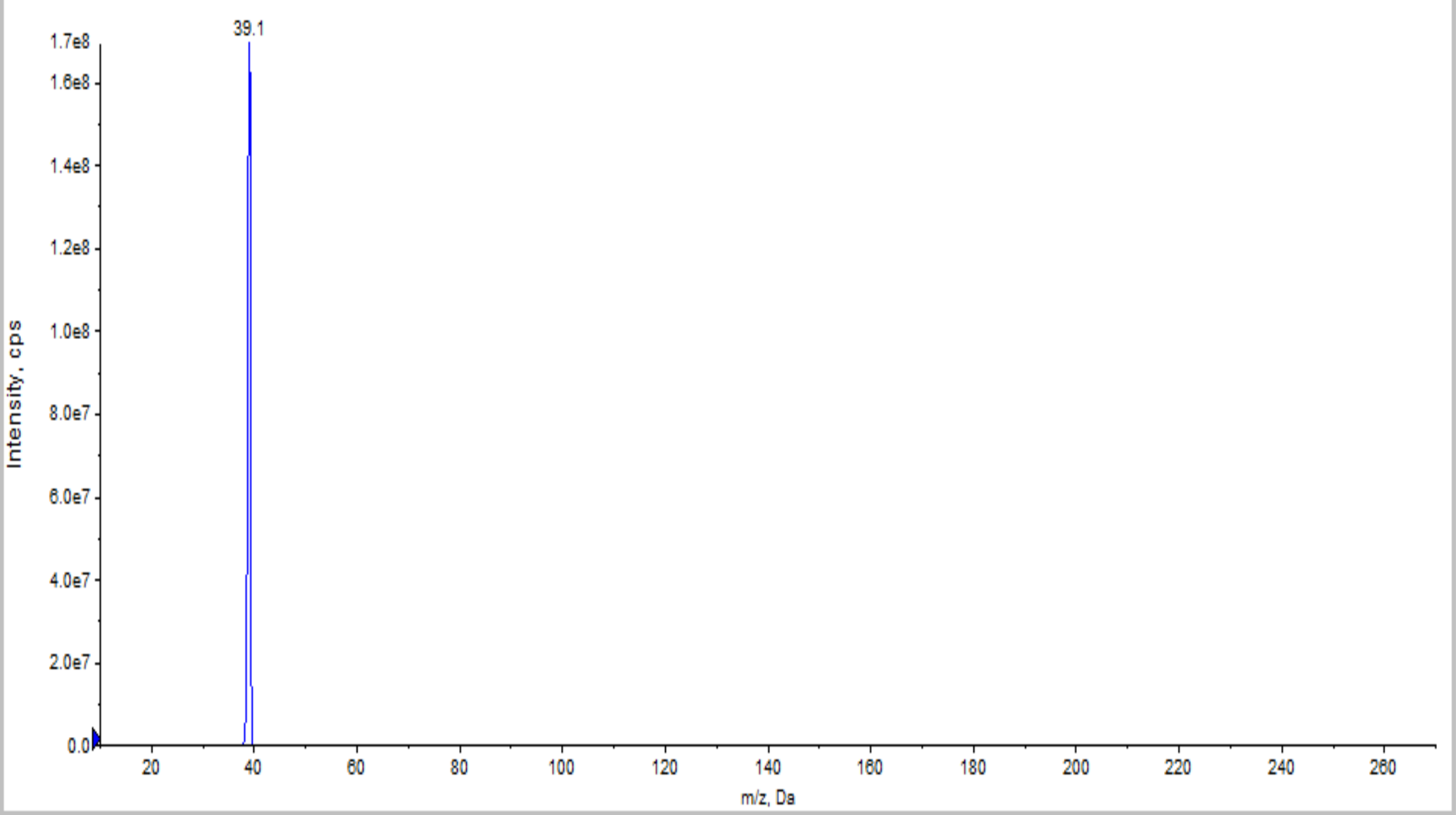

Figure C4.6 A-D Product ion spectra used for the identification of potassium complex formation.

A. PI $m / z 259-\left[15-5+{ }^{39} \mathrm{~K}\right]^{+}, m / z, 39-\left[{ }^{39} \mathrm{~K}\right]^{+}$ 


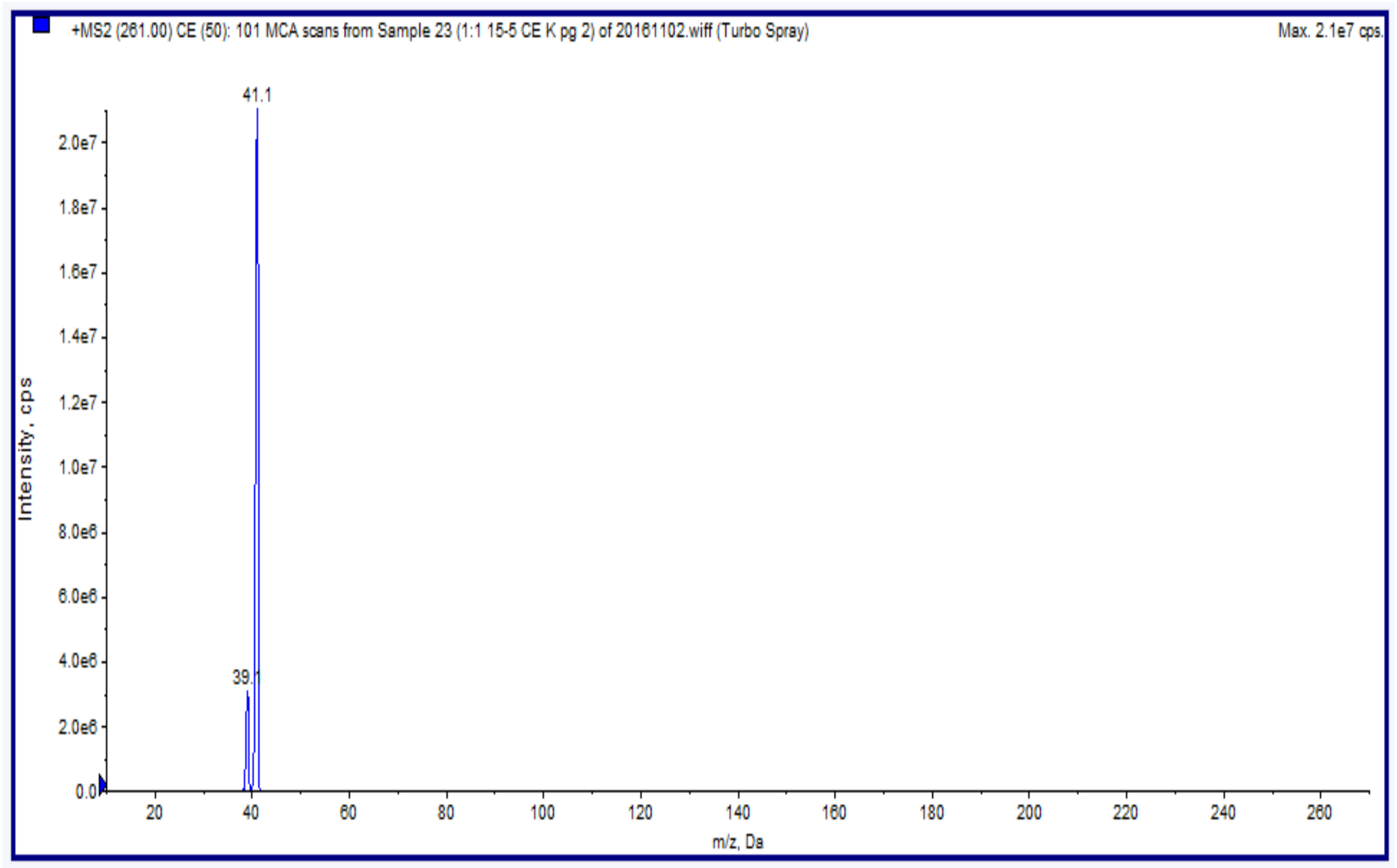

B. PI $m / z 261-\left[15-5+{ }^{41} \mathrm{~K}\right]^{+}, m / z 41-\left[{ }^{41} \mathrm{~K}\right]^{+}$

Figure C4.6 A-D Continued 


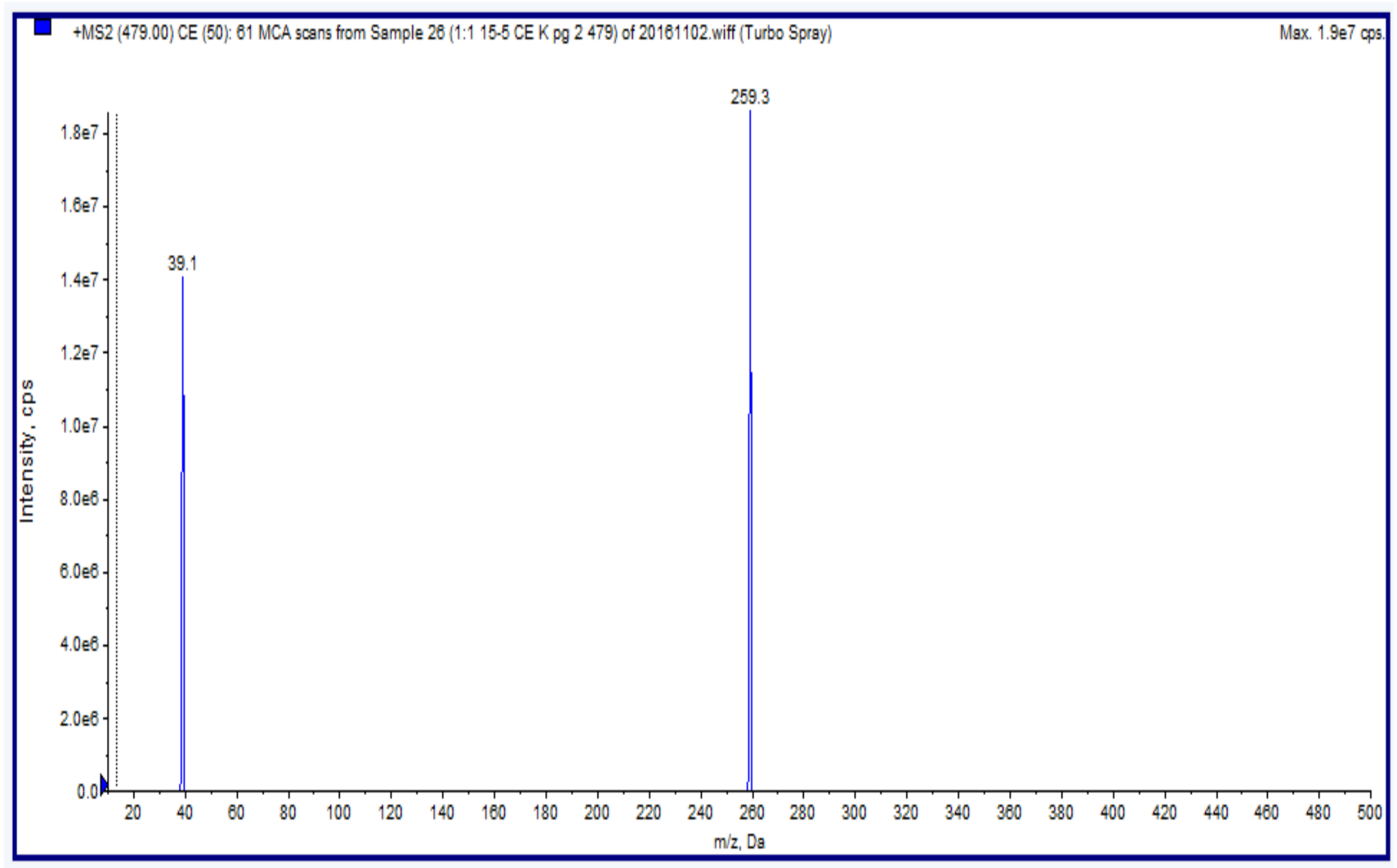

C. PI $m / z 479-\left[15-52+{ }^{39} \mathrm{~K}\right]^{+}, m / z, 259-\left[15-5+{ }^{39} \mathrm{~K}\right]^{+}, m / z, 39-\left[{ }^{39} \mathrm{~K}\right]^{+}$

Figure C4.6 A-D Continued 


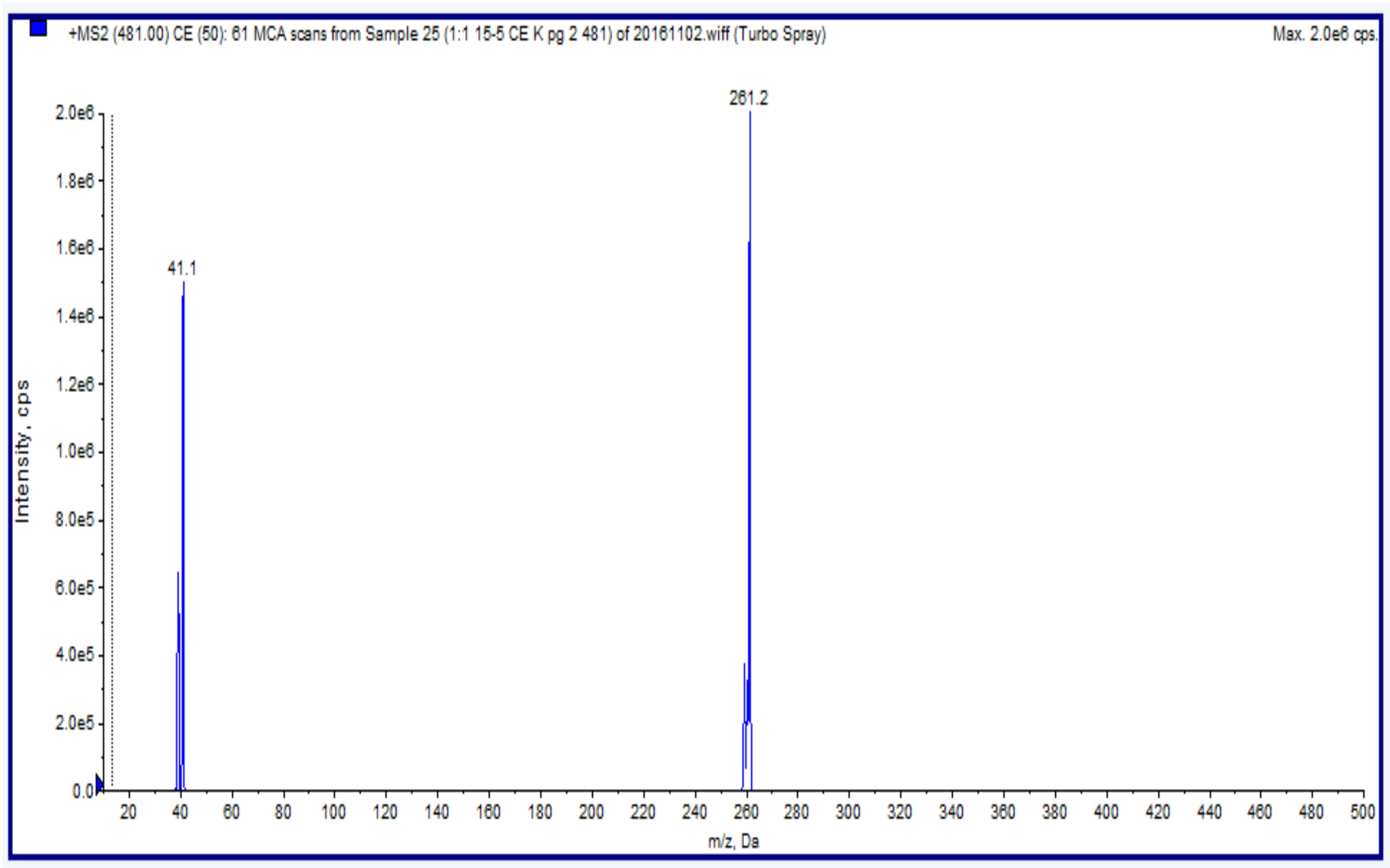

D. PI $m / z 481-\left[15-5_{2}+{ }^{41} \mathrm{~K}\right]^{+}, m / z 261-\left[15-5+{ }^{41} \mathrm{~K}\right]^{+}, m / z 41-\left[{ }^{41} \mathrm{~K}\right]^{+}$

Figure C4.6 A-D Continued 
Table C4.1 The peaks used to calculate \% $\mathrm{T}[\mathrm{M}]$ for each calculation method utilized.

\begin{tabular}{|c|c|c|c|c|}
\hline Method & $\mathbf{C u}$ & $\mathbf{B a}$ & $\mathbf{P b}$ & $\mathbf{K}$ \\
\hline $\begin{array}{l}\text { Method 1: Most Abundant } \\
\text { Isotope in Favored Complex }\end{array}$ & {$\left[15-5+{ }^{63} \mathrm{Cu}\right]^{+}$} & {$\left[15-5+{ }^{138} \mathrm{Ba}+\mathrm{NO}_{3}\right]^{+}$} & {$\left[15-5+{ }^{208} \mathrm{~Pb}+\mathrm{NO}_{3}\right]^{+}$} & $\begin{array}{c}{\left[15-5+{ }^{39} \mathrm{~K}\right]^{+} \text {or }} \\
{\left[15-5+{ }^{41} \mathrm{~K}\right]^{+}}\end{array}$ \\
\hline \multirow{2}{*}{$\begin{array}{l}\text { Method 2: Most Abundant } \\
\text { Isotope in the Both Complexes }\end{array}$} & {$\left[15-5+{ }^{63} \mathrm{Cu}\right]^{+}$} & {$\left[15-5+{ }^{138} \mathrm{Ba}+\mathrm{NO}_{3}\right]^{+}$} & {$\left[15-5+{ }^{208} \mathrm{~Pb}+\mathrm{NO}_{3}\right]^{+}$} & $\begin{array}{c}{\left[15-5+{ }^{39} \mathrm{~K}\right]^{+} \text {or }} \\
{\left[15-5+{ }^{41} \mathrm{~K}\right]^{+}}\end{array}$ \\
\hline & {$\left[15-5+{ }^{63} \mathrm{Cu}+\mathrm{NO}_{3}\right]^{+}$} & {$\left[15-5_{2}+{ }^{138} \mathrm{Ba}+\mathrm{NO}_{3}\right]^{+}$} & {$\left[15-5_{2}+{ }^{208} \mathrm{~Pb}+\mathrm{NO}_{3}\right]^{+}$} & $\begin{array}{c}{\left[15-5_{2}+{ }^{39} \mathrm{~K}\right]^{+} \text {or }} \\
{\left[15-5_{2}+{ }^{41} \mathrm{~K}\right]^{+}}\end{array}$ \\
\hline \multirow{5}{*}{$\begin{array}{l}\text { Method 3: All Isotopes in } \\
\text { Favored Complex }\end{array}$} & {$\left[15-5+{ }^{63} \mathrm{Cu}\right]^{+}$} & {$\left[15-5+{ }^{134} \mathrm{Ba}+\mathrm{NO}_{3}\right]^{+}$} & {$\left[15-5+{ }^{204} \mathrm{~Pb}+\mathrm{NO}_{3}\right]^{+}$} & {$\left[15-5+{ }^{39} \mathrm{~K}\right]^{+}$} \\
\hline & {$\left[15-5+{ }^{65} \mathrm{Cu}\right]^{+}$} & {$\left[15-5+{ }^{135} \mathrm{Ba}+\mathrm{NO}_{3}\right]^{+}$} & {$\left[15-5+{ }^{206} \mathrm{~Pb}+\mathrm{NO}_{3}\right]^{+}$} & {$\left[15-5+{ }^{41} \mathrm{~K}\right]^{+}$} \\
\hline & & {$\left[15-5+{ }^{136} \mathrm{Ba}+\mathrm{NO}_{3}\right]^{+}$} & {$\left[15-5+{ }^{207} \mathrm{~Pb}+\mathrm{NO}_{3}\right]^{+}$} & \\
\hline & & {$\left[15-5+{ }^{137} \mathrm{Ba}+\mathrm{NO}_{3}\right]^{+}$} & {$\left[15-5+{ }^{208} \mathrm{~Pb}+\mathrm{NO}_{3}\right]^{+}$} & \\
\hline & & {$\left[15-5+{ }^{138} \mathrm{Ba}+\mathrm{NO}_{3}\right]^{+}$} & & \\
\hline \multirow{8}{*}{$\begin{array}{l}\text { Method 4: All Isotopes in Both } \\
\text { Complexes }\end{array}$} & {$\left[15-5+{ }^{63} \mathrm{Cu}\right]^{+}$} & {$\left[15-5+{ }^{134} \mathrm{Ba}+\mathrm{NO}_{3}\right]^{+}$} & {$\left[15-5+{ }^{204} \mathrm{~Pb}+\mathrm{NO}_{3}\right]^{+}$} & {$\left[15-5+{ }^{39} \mathrm{~K}\right]^{+}$} \\
\hline & {$\left[15-5+{ }^{65} \mathrm{Cu}\right]^{+}$} & {$\left[15-5+{ }^{135} \mathrm{Ba}+\mathrm{NO}_{3}\right]^{+}$} & {$\left[15-5+{ }^{206} \mathrm{~Pb}+\mathrm{NO}_{3}\right]^{+}$} & {$\left[15-5+{ }^{41} \mathrm{~K}\right]^{+}$} \\
\hline & {$\left[15-5+{ }^{63} \mathrm{Cu}+\mathrm{NO}_{3}\right]^{+}$} & {$\left[15-5+{ }^{136} \mathrm{Ba}+\mathrm{NO}_{3}\right]^{+}$} & {$\left[15-5+{ }^{207} \mathrm{~Pb}+\mathrm{NO}_{3}\right]^{+}$} & {$\left[15-5_{2}+{ }^{39} \mathrm{~K}\right]^{+}$} \\
\hline & {$\left[15-5+{ }^{65} \mathrm{Cu}+\mathrm{NO}_{3}\right]^{+}$} & {$\left[15-5+{ }^{137} \mathrm{Ba}+\mathrm{NO}_{3}\right]^{+}$} & {$\left[15-5+{ }^{208} \mathrm{~Pb}+\mathrm{NO}_{3}\right]^{+}$} & {$\left[15-5_{2}+{ }^{41} \mathrm{~K}\right]^{+}$} \\
\hline & & {$\left[15-5+{ }^{138} \mathrm{Ba}+\mathrm{NO}_{3}\right]^{+}$} & {$\left[15-5_{2}+{ }^{206} \mathrm{~Pb}+\mathrm{NO}_{3}\right]^{+}$} & \\
\hline & & {$\left[15-5_{2}+{ }^{135} \mathrm{Ba}+\mathrm{NO}_{3}\right]^{+}$} & {$\left[15-5_{2}+{ }^{207} \mathrm{~Pb}+\mathrm{NO}_{3}\right]^{+}$} & \\
\hline & & {$\left[15-5_{2}+{ }^{136} \mathrm{Ba}+\mathrm{NO}_{3}\right]^{+}$} & {$\left[15-5_{2}+{ }^{208} \mathrm{~Pb}+\mathrm{NO}_{3}\right]^{+}$} & \\
\hline & & {$\left[15-5_{2}+{ }^{137} \mathrm{Ba}+\mathrm{NO}_{3}\right]^{+}$} & & \\
\hline
\end{tabular}

